Aus der Abteilung Psychosomatische Medizin und Psychotherapie

(Prof. Dr. med. C. Herrmann-Lingen)

im Zentrum Psychosoziale Medizin

der medizinischen Fakultät der Universität Göttingen

\title{
Depressivität, Angst, Vitale Erschöpfung, Lebensqualität und ihr Zusammenhang mit Herzfrequenzvariabilität bei Patienten mit kardiovaskulären Risikofaktoren
}

\author{
INAUGURAL - DISSERTATION \\ zur Erlangung des Doktorgrades \\ der medizinischen Fakultät \\ der Georg-August-Universität zu Göttingen \\ vorgelegt von \\ Vera Charlotte Duden
}

aus

Heidelberg 
DEKAN: Prof. Dr. med. C. Frömmel

I. Berichterstatter: Prof. Dr. med. C. Herrmann-Lingen

II. Berichterstatter/in:

III. Berichterstatter/in:

Tag der mündlichen Prüfung: 


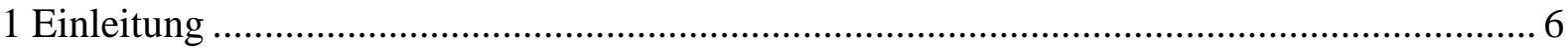

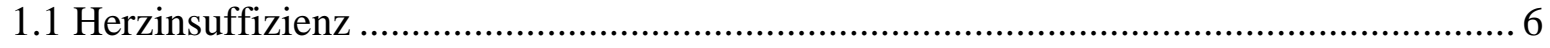

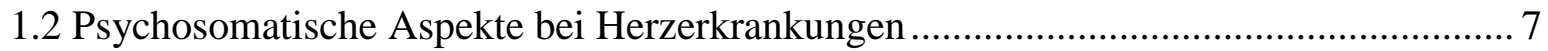

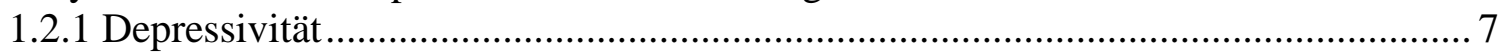

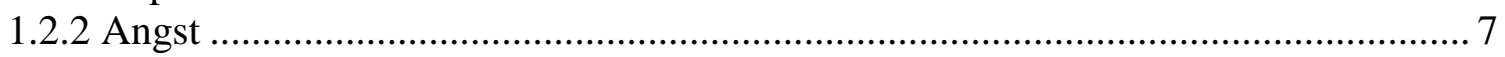

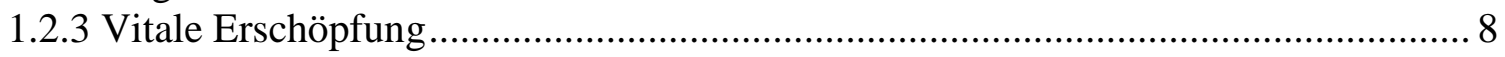

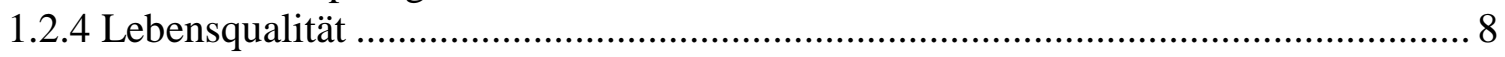

1.3 Herzfrequenzvariabilität als Ausdruck autonomer Regulation ................................... 9

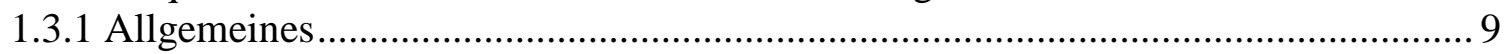

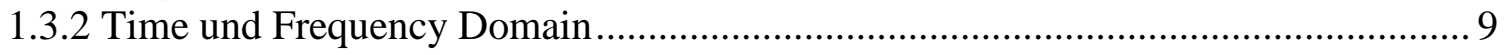

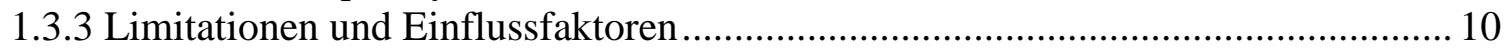

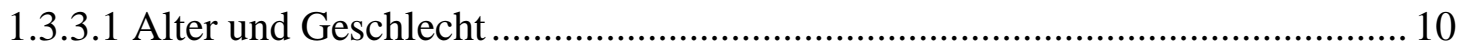

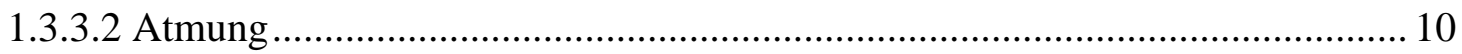

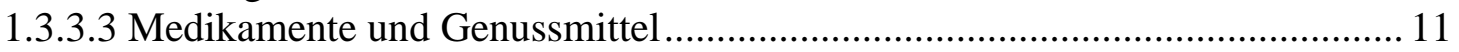

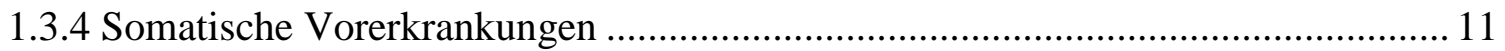

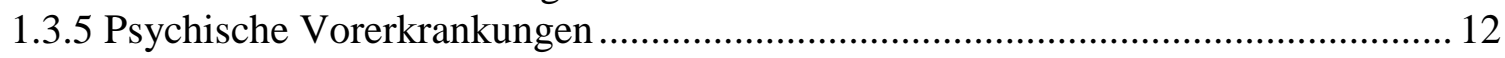

1.4 Kardiale Schweregradmarker und ihr Zusammenhang mit HRV und Psyche ................ 13

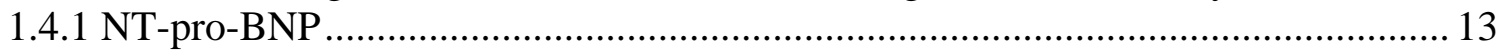

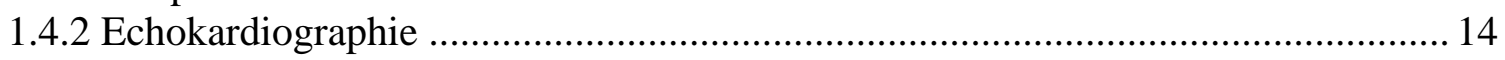

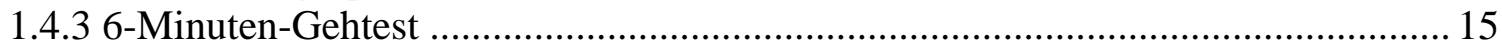

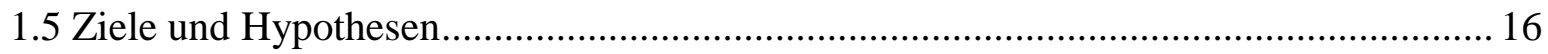

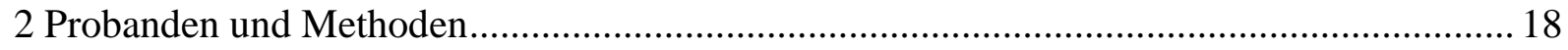

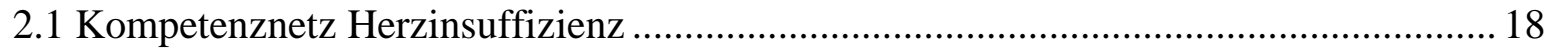

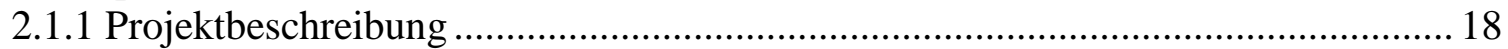

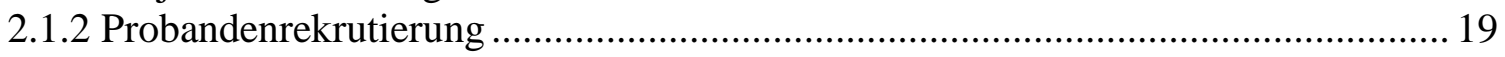

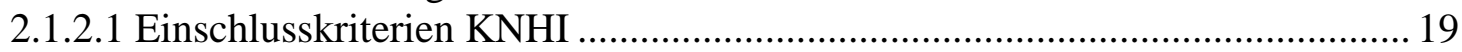

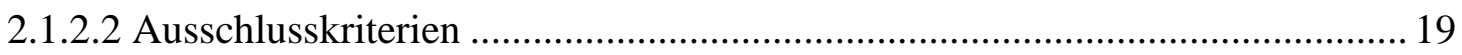

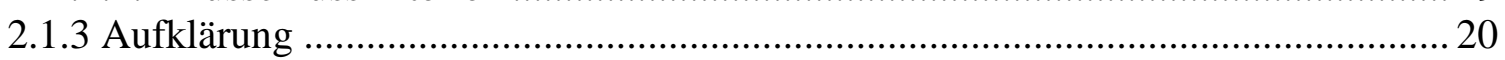

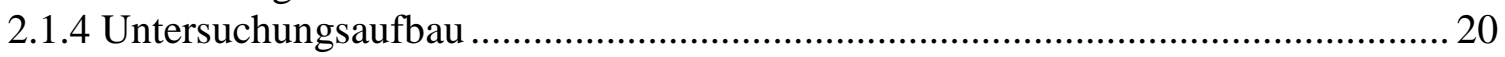

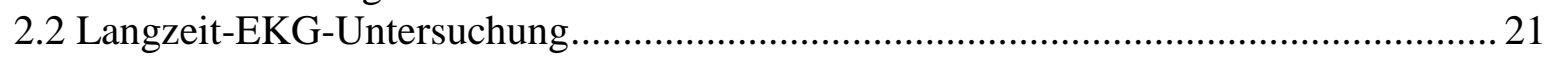

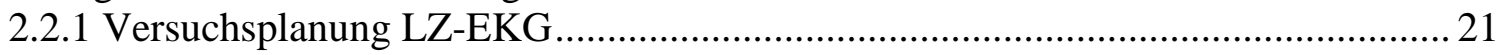

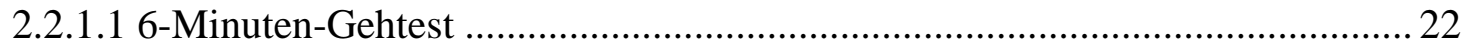

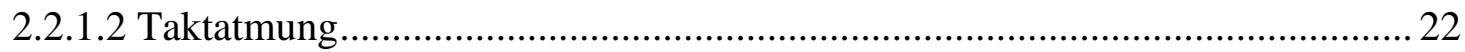

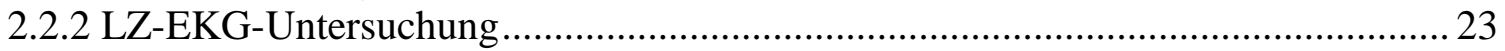

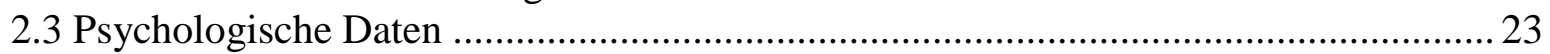

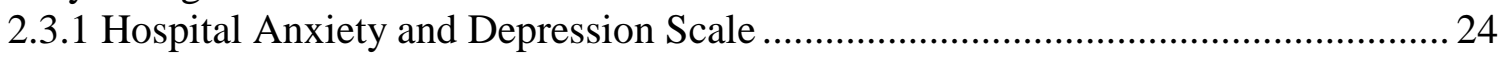

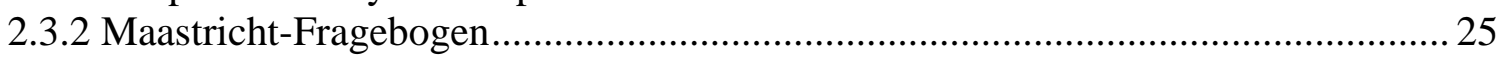

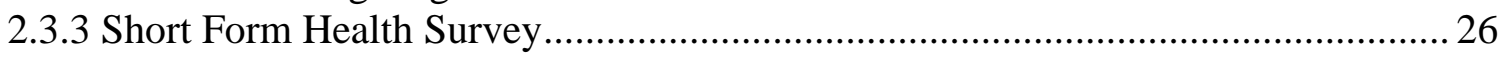

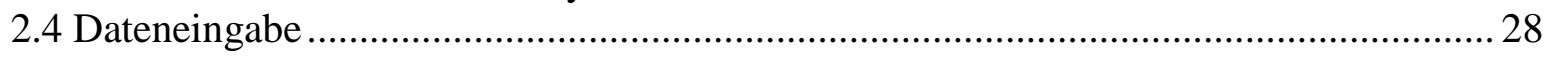

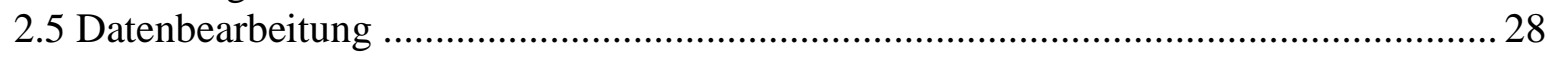

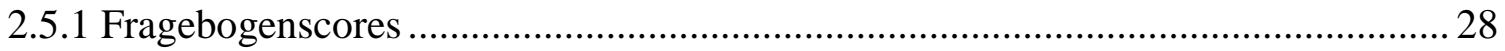

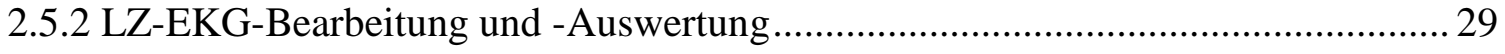

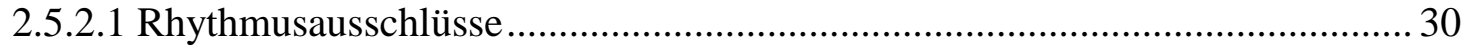

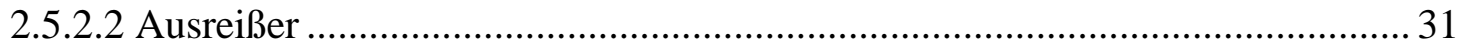

2.5.2.3 Ausschlüsse von EKG-Phasen ................................................................... 31

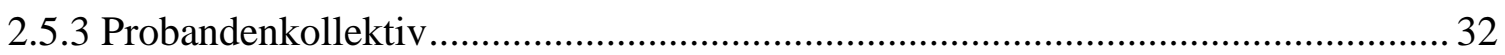

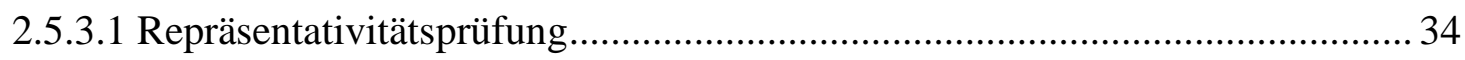

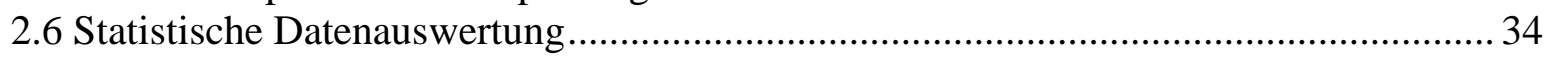

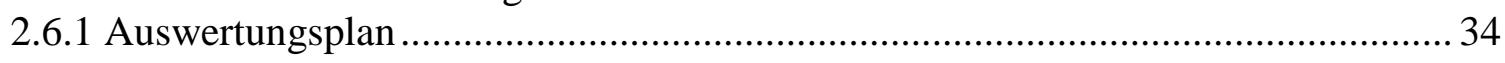

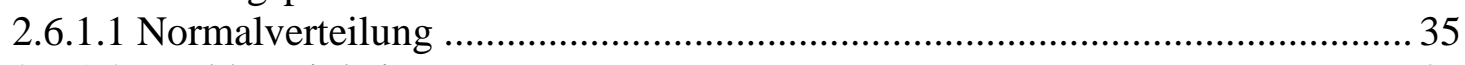

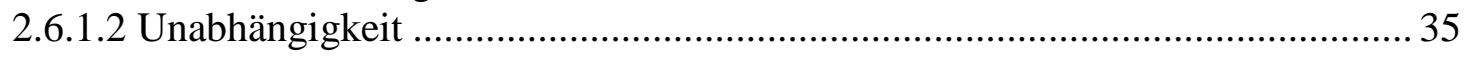




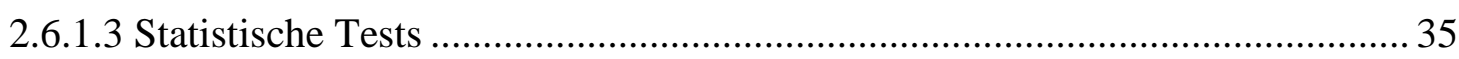

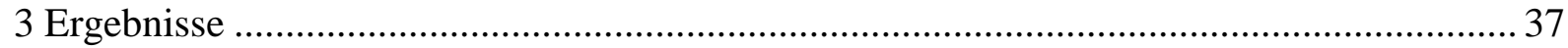

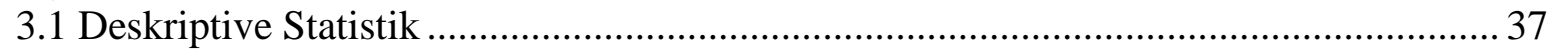

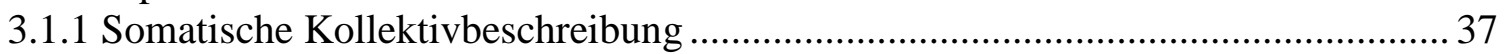

3.1.1.1 Kardiale Vorerkrankungen, Risikofaktoren, Medikamente ............................ 37

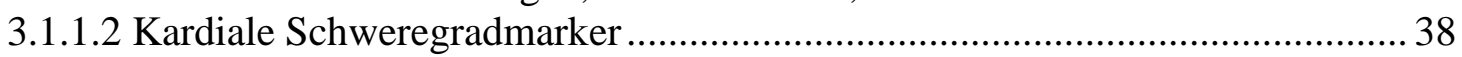

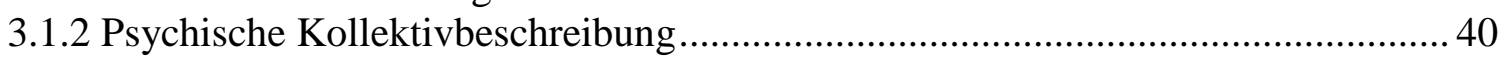

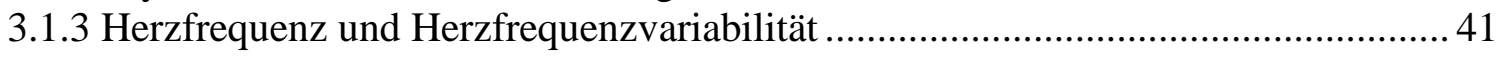

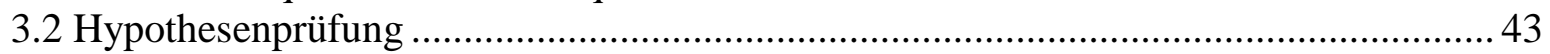

3.2.1 Zusammenhang zwischen Depressivität und HR sowie HRV-Parametern

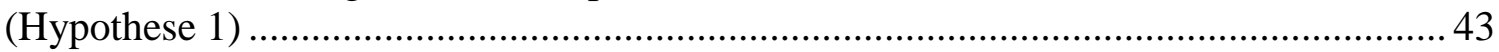

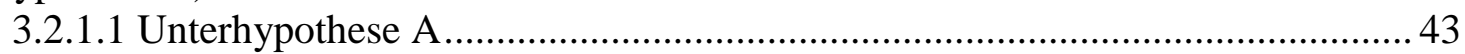

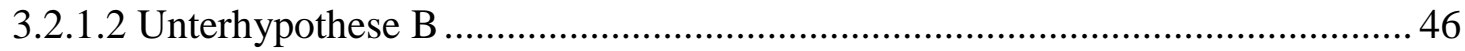

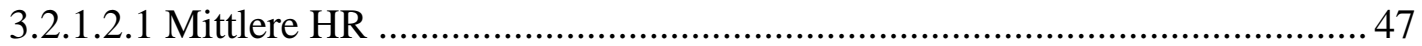

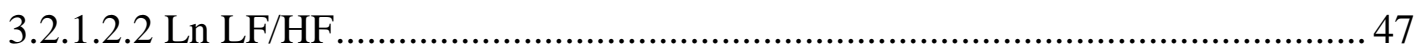

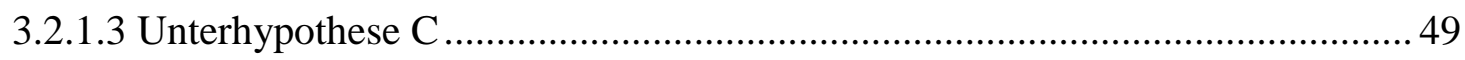

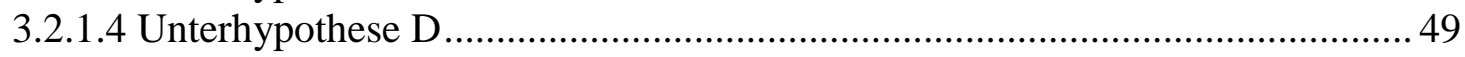

3.2.2 Zusammenhang zwischen Angst, Vitaler Erschöpfung, körperlicher

Funktionsfähigkeit und HR sowie HRV-Parametern (Hypothese 2) ...............................50

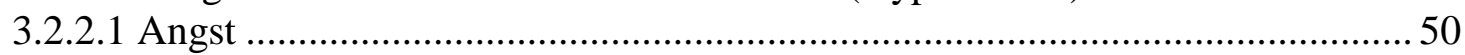

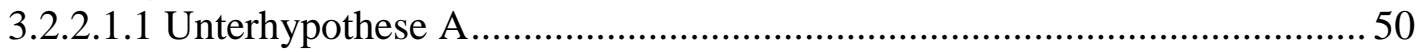

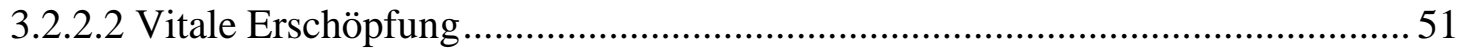

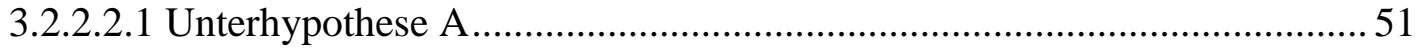

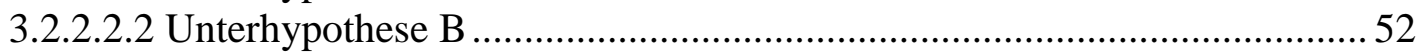

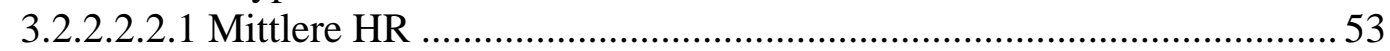

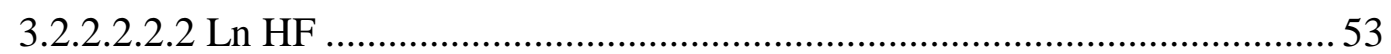

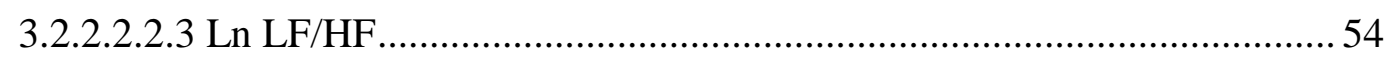

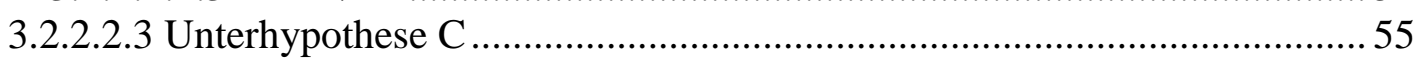

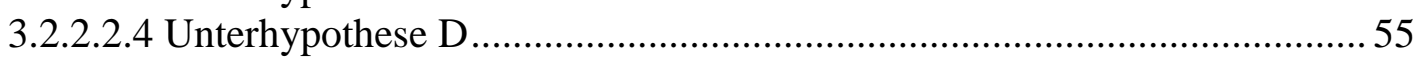

3.2.2.3 Körperliche Funktionsfähigkeit des SF-36 ............................................. 56

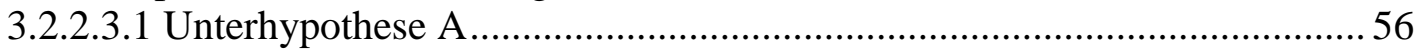

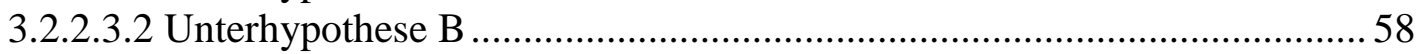

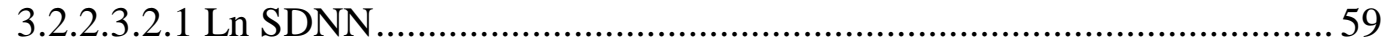

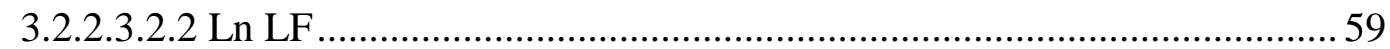

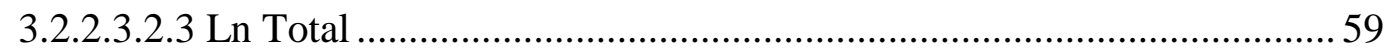

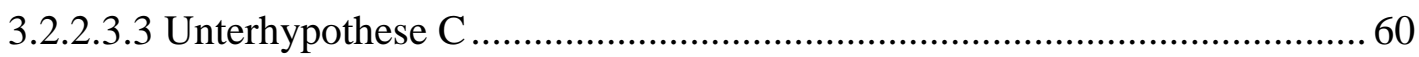

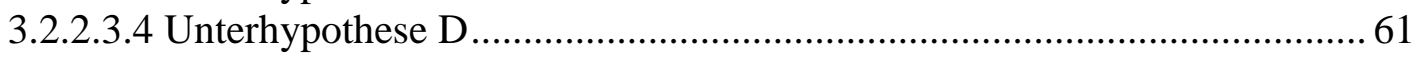

3.2.3 Zusammenhang zwischen kardialer Vorerkrankung, kardialen Schweregradmarkern

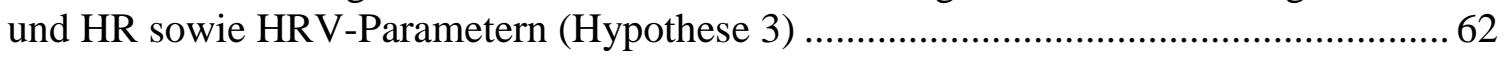
3.2.4 Zusammenhang zwischen 6-Minuten-Gehteststrecke und HR und HRV-Parametern

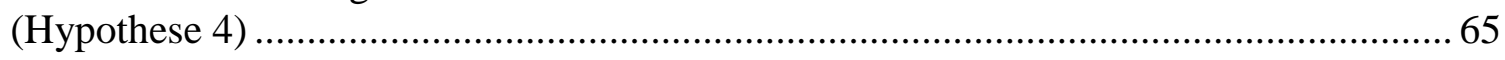

3.2.5 Zusammenhang zwischen psychologischen Variablen und kardialen

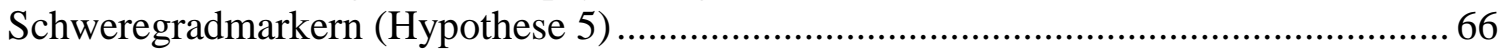

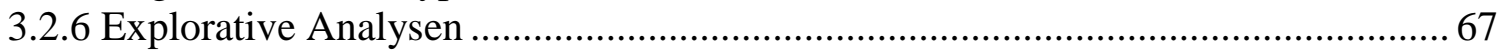

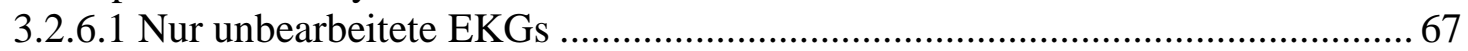

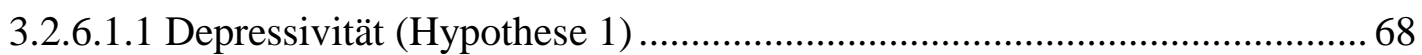

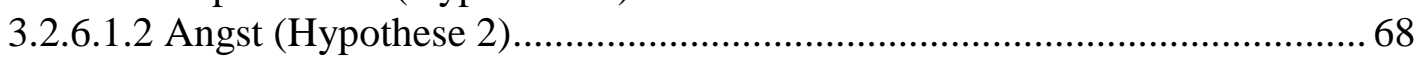

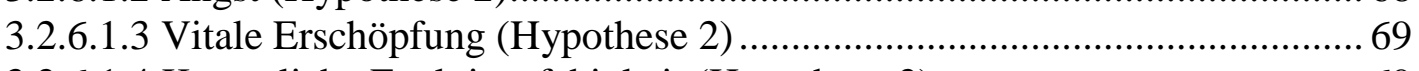

3.2.6.1.4 Körperliche Funktionsfähigkeit (Hypothese 2) ....................................... 69

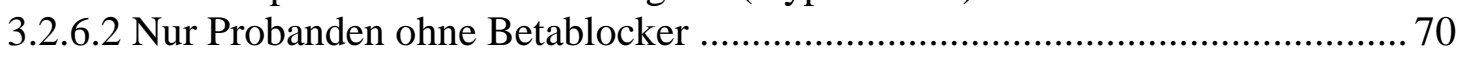

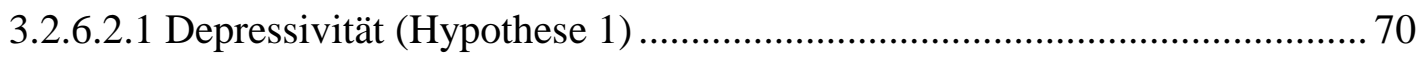




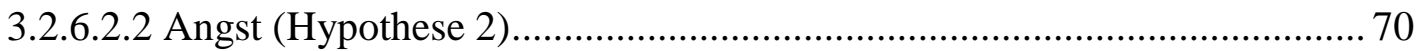

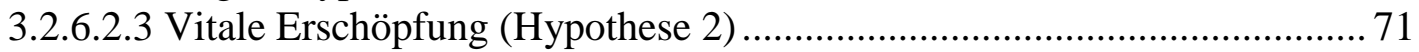

3.2.6.2.4 Körperliche Funktionsfähigkeit (Hypothese 2) ..................................... 71

4 Diskussion

4.1 Zusammenhänge von HRV-Parametern mit kardialen Vorerkrankungen und kardialen

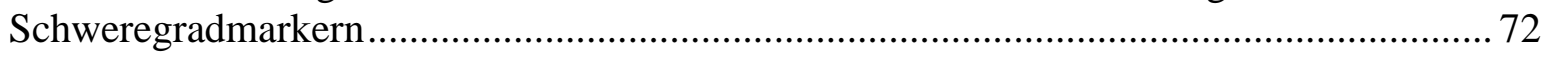

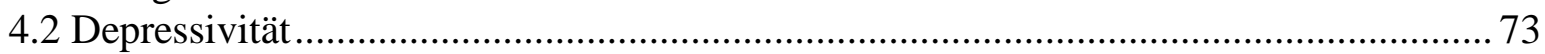

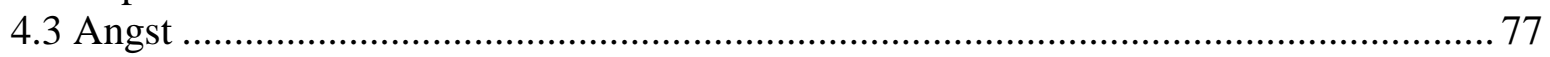

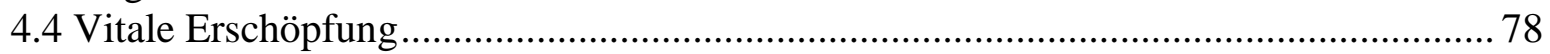

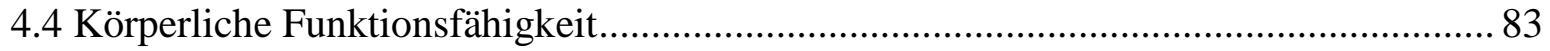

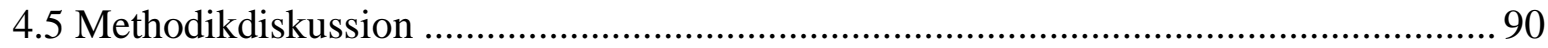

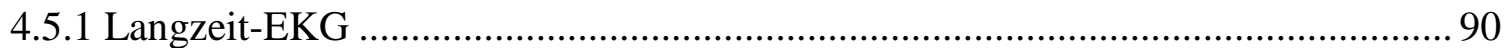

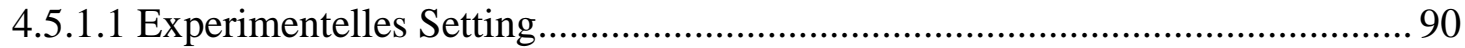

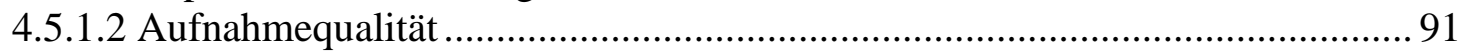

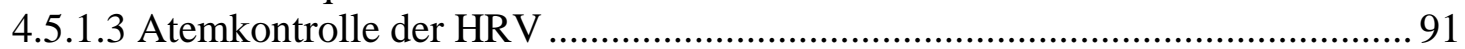

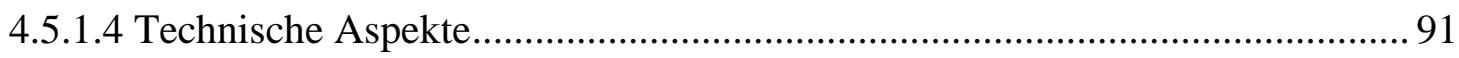

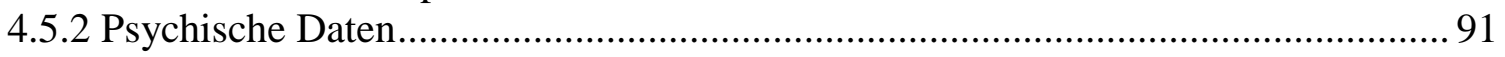

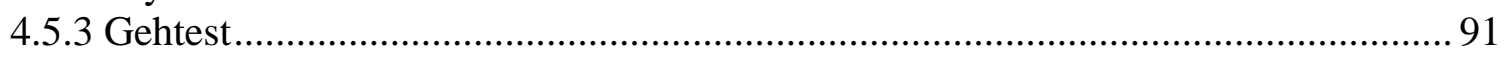

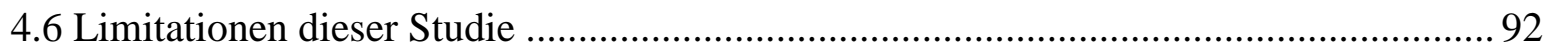

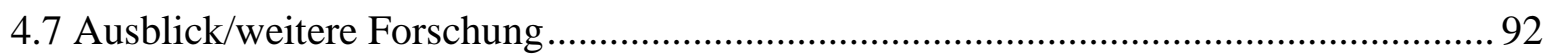

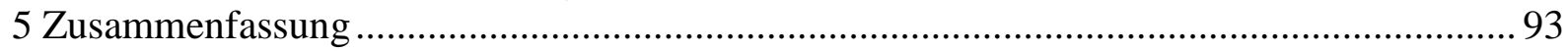

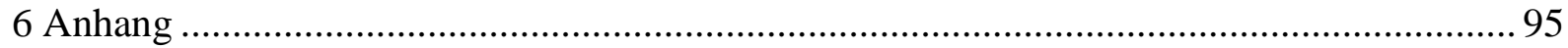

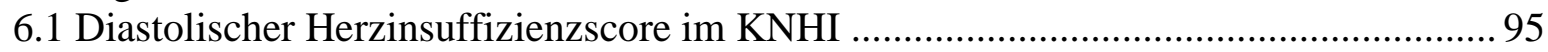

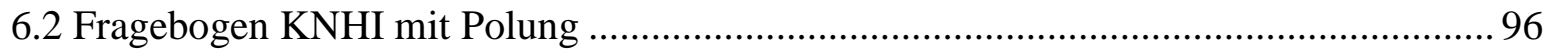

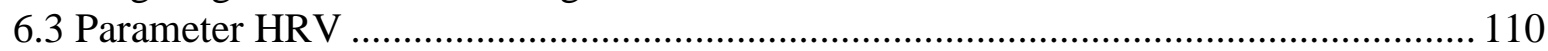

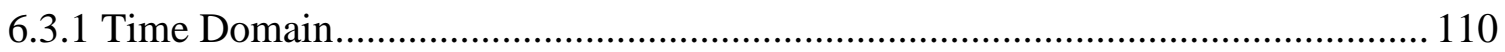

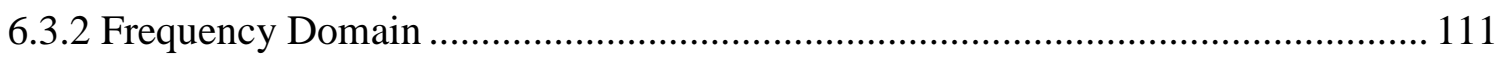

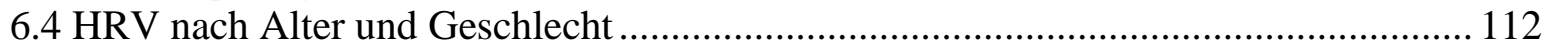

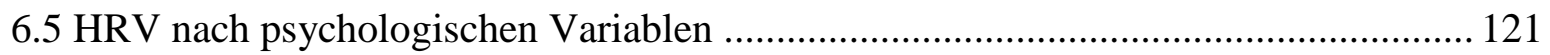

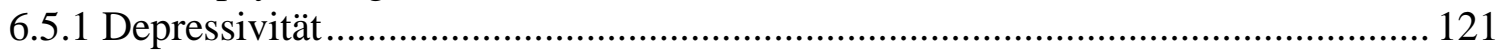

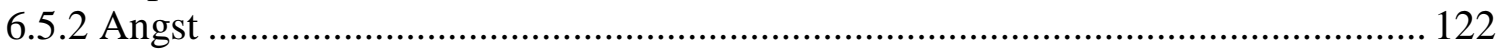

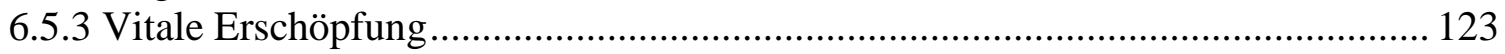

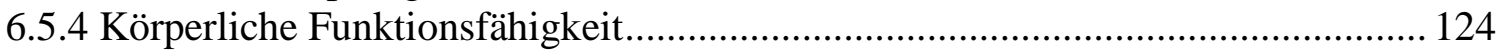

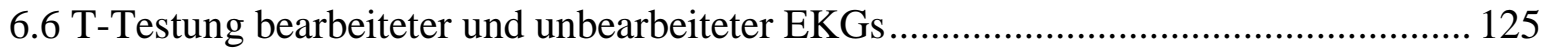

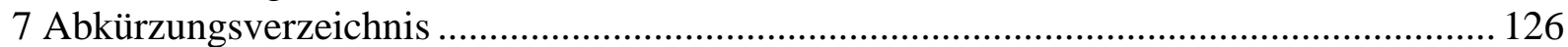

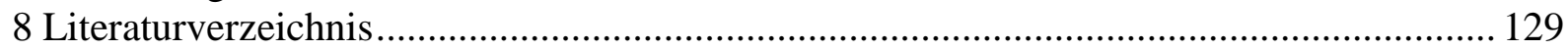




\section{Einleitung}

Für depressive Herzkranke wird eine erhöhte Mortalitätsrate beschrieben (CARNEY und FREEDLAND 2009; LADWIG et al. 2008).

Für diesen Zusammenhang wird neben anderen Faktoren eine Fehlregulation des autonomen Nervensystems (ANS) mit vermehrter Sympathikus- und verminderter Parasympathikusaktivierung als Erklärungsmodell angenommen. Das ANS kann mittels Herzfrequenzvariabilitätsanalyse untersucht werden (CARNEY und FREEDLAND 2009; HENNERSDORF und STRAUER 2002). Diese Promotionsstudie prüft den Zusammenhang von Depressivität, Angst, Vitaler Erschöpfung, körperlicher Lebensqualität und der Herzfrequenzvariabilität (HRV) in drei unterschiedlichen Aktivierungszuständen des ANS an einem Herzinsuffizienzund Herzinsuffizienzrisikokollektiv im Rahmen des Kompetenznetzes Herzinsuffizienz.

\subsection{Herzinsuffizienz}

Aus vielen Herzerkrankungen kann im ungünstigen Fall für den Patienten eine Herzinsuffizienz (HI) entstehen (RAUCHHAUS 2004). Das Herz ist bei einer Herzinsuffizienz nicht mehr in der Lage, den Körper in Ruhe und unter Belastung mit ausreichend Sauerstoff zu versorgen. Man unterscheidet systolische und diastolische Herzinsuffizienz. Eingeteilt und beurteilt wird Herzinsuffizienz anhand von subjektiven (NYHA-Klasse) und objektiven (z. B. Echokardiographie, NT-pro-BNP, 6-min-Gehtest) Parametern. Teil der physiologischen Grundlage der Herzinsuffizienz ist eine in der Echokardiographie erkennbare Dysfunktion. Hierbei unterscheidet man ebenfalls eine systolische und eine diastolische Dysfunktion (ANGERMANN und ERTL 2004), die jeweils mit unterschiedlichen Vorerkrankungen verbunden sind und eine unterschiedliche Verteilung zwischen den Geschlechtern haben (SCHEELE 2011).

Die Inzidenz und Prävalenz der Herzinsuffizienz (s. SCHEELE 2011: kardiologische Doktorarbeit der KNHI-Studie) steigen aufgrund der verbesserten Therapie von kardialen Akuterkrankungen (RAUCHHAUS 2004). Im Jahre 2006 war die Herzinsuffizienz in Deutschland nach der Einteilung der „International Statistical Classification of Diseases and Related Health Problems“ (ICD) nach der Diagnose „Neugeborenes“ die häufigste Hauptdiagnose der stationären Aufenthalte mit mehr als 300.000 Fällen (STATISTISCHES BUNDESAMT 2007). Bei den Todesursachen nach ICD steht die Herzinsuffizienz an zweiter (Frauen) beziehungsweise vierter (Männer) Stelle (ROBERT-KOCH-INSTITUT 2006). 
Sowohl auf den Krankheitseintritt als auch auf den Krankheitsverlauf haben psychische Faktoren großen Einfluss (LADWIG et al. 2008). Mit diesen Zusammenhängen beschäftigt sich die Psychokardiologie.

\subsection{Psychosomatische Aspekte bei Herzerkrankungen}

Depression, Angst und Vitale Erschöpfung (VE) scheinen besonders starken Einfluss auf die Entstehung und den Verlauf kardialer Erkrankungen zu haben. Für die Patienten besonders relevant ist die subjektive Lebensqualität, die bei kardialen Erkrankungen erniedrigt ist (LADWIG et al. 2008).

\subsubsection{Depressivität}

Bei somatisch erkrankten Patienten ist eine Depression eine häufige Komorbidität, wobei es sich oft um leichtere Ausprägungen von Depressivität handelt. Die Prävalenz von depressiven Störungen liegt bei kardiologischen Patienten zwischen 15 und 20\%, für Diabetiker bei bis zu $38 \%$ im Vergleich zu $6 \%$ bei allgemeinmedizinisch-internistischen Patienten in der hausärztlichen Betreuung (HERRMANN-LINGEN 2003). Von den ambulanten Herzinsuffizienzpatienten leiden 11-25\% an einer depressiven Störung. Im stationären Bereich sind es 35-70\% (LADWIG et al. 2008). Prognostisch ist Depressivität bei kardialer Vorerkrankung mit erhöhter Mortalität verbunden (LADWIG et al. 2008).

\subsubsection{Angst}

In der Allgemeinbevölkerung liegt die Lebenszeitprävalenz aller Angststörungen bei circa 15\% (MEYER C et al. 2000). Prognostisch ist Angst teilweise für beide Geschlechter als ein Risikofaktor für erhöhte Morbidität und Mortalität an kardiovaskulären Erkrankungen beschrieben worden (ROEST et al. 2010a; ROEST et al. 2010b; LADWIG et al. 2008; JANUZZI et al. 2000). Teilweise wird eine erniedrigte Mortalitätsrate im Zusammenhang mit Angst beobachtet (HERRMANN et al. 2000). Die negativen Auswirkungen der Angst beruhen vermutlich auf einem physiologischen Arousal bei Angst als einer potentiellen Ursache für kardiale Verschlechterung z. B. durch Arrhythmien (z. B. MACMAHON und LIP 2002). Eine positive Wirkung der Angst ist in einer frühzeitigen Kontaktaufnahme und damit früheren Diagnose und Therapie begründet. Am ehesten ist diese Spannweite der Beobachtungen bezüglich Angst durch die unterschiedlichen Ausprägungen von Angstsymptomatik (HERR- 
MANN et al. 2000), aber auch durch eine Interaktion der Angst mit den kardialen Vorerkrankungen bedingt (MEYER T et al. 2010).

\subsubsection{Vitale Erschöpfung}

Ein der Depression ähnliches Konstrukt ist das der Vitalen Erschöpfung (VE). APPELS et al. 2000 beschreiben es als das depressive Symptom mit der höchsten Korrelation zur Mortalität (APPELS et al. 2000). Entwickelt wurde das Konzept aus der Beobachtung heraus, dass Patienten vor einem Herzinfarkt eine Phase von annähernd 12 Monaten mit Erschöpfungsgefühl erleben (APPELS 2004). Die Prävalenz der Vitalen Erschöpfung liegt bei kardiologischen Patienten zwischen 35 und 60\% (KOP 1999). Für beide Geschlechter ist die VE ein ungünstiger prognostischer Faktor für Morbidität und Mortalität an kardiovaskulären Erkrankungen (LADWIG et al. 2008; APPELS 2004; KOERTGE 2003).

\subsubsection{Lebensqualität}

Das Konstrukt der Lebensqualität wurde ab den 1970ern als Messparameter zur Beschreibung der subjektiven Gesundheit eingeführt (BULLINGER 1997) und in der Regel als multidimensionales Konzept angesehen, wobei unter anderem psychisches Befinden, körperliche Verfassung, funktionale Kompetenz und soziale Beziehungen berücksichtigt werden (BULLINGER und KIRCHBERGER 1998). In der vorliegenden Arbeit wurde nur der Aspekt der körperlichen Funktionsfähigkeit näher betrachtet.

Bei kardiologischen Patienten ist die Lebensqualität, insbesondere auch die körperliche Funktionsfähigkeit erniedrigt (LADWIG 2008; ALONSO et al. 2004; JUENGER et al. 2002). Prognostisch wird ein Zusammenhang von niedriger Lebensqualität mit erhöhter Mortalität vermutet (LADWIG et al. 2008).

Insgesamt haben Depression, Angst, Vitale Erschöpfung und Lebensqualität Einfluss auf den Verlauf einer kardiovaskulären Erkrankung und sind teilweise klar als ungünstige Prognosefaktoren identifiziert worden. 


\subsection{Herzfrequenzvariabilität als Ausdruck autonomer Regulation}

Die HRV beschreibt die Modulationen des autonomen Nervensystems am herzeigenen Erregungsbildungs- und Leitungssystem (TASK FORCE OF THE EUROPEAN SOCIETY OF CARDIOLOGY AND THE NORTH AMERICAN SOCIETY OF PACING AND ELECTROPHYSIOLOGY 1996). Eine autonome Dysfunktion, in dieser Arbeit definiert als Ungleichgewicht der autonomen Nervensysteme mit Überaktivität des Sympathikus und verminderter Aktivität des Parasympathikus, zeigt sich anhand von erniedrigten HRVParametern (HENNERSDORF und STRAUER 2002). Eine erniedrigte HRV, unabhängig von der Ursache, ist mit einer erhöhten Mortalität verbunden (SINSKI et al. 2006; SZTAJZEL 2004). Für die beobachtete erhöhte Mortalitätsrate bei psychischen Begleiterkrankungen im Rahmen von kardialen Erkrankungen (LADWIG et al. 2008) wird unter anderem die autonome Dysfunktion, messbar mittels HRV, als Erklärungsmodell herangezogen (z. B. CARNEY und FREEDLAND 2009).

\subsubsection{Allgemeines}

Die Messung der Herzfrequenz (HR) und der HRV-Parameter ist eine schnelle, kostengünstige, nicht-invasive Methode, um über den autonomen Status eines Patienten Auskunft zu erhalten. Diese Methode wird in der Forschung und einigen speziellen Indikationen in der Kardiologie genutzt, z. B. nach Myokardinfarkt oder bei Diabetes mellitus (RAJENDRA ACHARYA et al. 2006). Gemessen wird in der Regel im Liegen oder Sitzen über wenige Minuten oder 24 Stunden. Fünfminütige Messzeiträume sind gut verwendbar und zeigen eine passable Korrelation mit den Werten über 24 Stunden (HENNERSDORF und STRAUER 2002; TASK FORCE OF THE EUROPEAN SOCIETY OF CARDIOLOGY AND THE NORTH AMERICAN SOCIETY OF PACING AND ELECTROPHYSIOLOGY 1996). Verschiedene Einflussfaktoren auf die HRV sind bekannt (RAJENDRA ACHARYA et al. 2006). Weitere Forschung erscheint insbesondere aufgrund der oft sehr komplexen Situation bei einzelnen Patienten weiterhin notwendig.

\subsubsection{Time und Frequency Domain}

Die HRV kann durch unterschiedliche Parameter dargestellt werden. Für diese Arbeit relevant sind die Parameter der Time und der Frequency Domain. 
Die Werte der Time Domain beschreiben die Herzfrequenz zu einem bestimmten Zeitpunkt oder den Abstand zwischen zwei benachbarten Normalschlägen (N). Aus den einfachen Time-Domain-Messwerten können komplexere statistische Parameter errechnet werden (TASK FORCE OF THE EUROPEAN SOCIETY OF CARDIOLOGY AND THE NORTH AMERICAN SOCIETY OF PACING AND ELECTROPHYSIOLOGY 1996; s. auch 6.3 Parameter HRV ab S.110).

Die Frequency Domain beschreibt das Herzsignal, zerlegt in die zugrunde liegenden sinusförmigen Schwingungen ihrer Frequenzen und die Amplituden dieser Schwingungen. Für Kurzzeitmessungen (2-5 Minuten) sind die Frequency-Domain-Parameter gut geeignet (YERAGANI 1995; TASK FORCE OF THE EUROPEAN SOCIETY OF CARDIOLOGY AND THE NORTH AMERICAN SOCIETY OF PACING AND ELECTROPHYSIOLOGY 1996; s. auch 6.3 Parameter HRV ab S.110).

\subsubsection{Limitationen und Einflussfaktoren}

Die HRV-Messung ist beschränkt auf Patienten im Sinusrhythmus und Patienten mit wenigen ektopischen Schlägen (SZTAJZEL 2004). Bei nicht-stationären Untersuchungsbedingungen sind vor allem die Frequency-Domain-Parameter in ihrer Aussagekraft kritisch zu beurteilen (TASK FORCE OF THE EUROPEAN SOCIETY OF CARDIOLOGY AND THE NORTH AMERICAN SOCIETY OF PACING AND ELECTROPHYSIOLOGY 1996).

Neben dem Rhythmus des Herzens haben verschiedene Faktoren Einfluss auf die HRV. Die für diese Arbeit relevantesten sind im Folgenden aufgeführt.

\subsubsection{Alter und Geschlecht}

Inzwischen scheint belegt zu sein, dass Alter und Geschlecht die HRV beeinflussen (RAJENDRA ACHARYA et al. 2006; s. auch 6.4 HRV nach Alter und Geschlecht ab S.112).

\subsubsection{Atmung}

Die HRV ist abhängig von der Atmung (RAJENDRA ACHARYA et al. 2006; TASK FORCE OF THE EUROPEAN SOCIETY OF CARDIOLOGY AND THE NORTH AMERICAN SOCIETY OF PACING AND ELECTROPHYSIOLOGY 1996), dabei insbesondere die „high frequency“ (HF), die als Maß für die respiratorische Sinusarrhythmie steht (FRENNEAUX 2004; s. auch 6.3 Parameter HRV ab S.110). 
Bei regelmäßigem Atmen in einer Frequenz nahe den Blutdruckwellen (Mayer-Wellen), die durch die pulsatilen Aktivitäten des Sympathikus circa alle 10 Sekunden entstehen (PAGANI et al.1997), kommt es zu einem „Resonanz-Effekt““ (gleichmäßige, sinusförmige Schwingungen maximaler Amplitude) (GOLENHOFEN und HILDEBRANDT 1958) und maximalen, durch die Atmung beeinflussten HRV-Werten (ZIEMSSEN et al. 2002). Die Anpassung der Atemfrequenz in Richtung der Blutdruckwellenfrequenz führt zu einer Steigerung der „low frequency“ (LF) (BERNARDI et al. 2000).

\subsubsection{Medikamente und Genussmittel}

Einige Medikamente haben Einfluss auf die HRV (TASK FORCE OF THE EUROPEAN SOCIETY OF CARDIOLOGY AND THE NORTH AMERICAN SOCIETY OF PACING AND ELECTROPHYSIOLOGY 1996). Die Vormedikation mit Betablockern zeigt diesbezüglich einen besonders ausgeprägten Effekt. Die vagalen HRV-Parameter werden erhöht, die sympathischen HRV-Parameter gesenkt als Zeichen für die Rückkehr der sympatho-vagalen Balance (RAJENDRA ACHARYA et al. 2006).

Rauchen erniedrigt die vagalen und erhöht die sympathischen HRV-Parameter (RAJENDRA ACHARYA et al. 2006). Akut hat der Konsum von Alkohol einen Effekt wie das Rauchen. Bei chronischem Alkoholabusus zeigen sich, wie bei Diabetes mellitus, Zeichen einer Neuropathie mit erniedrigter HRV (RAJENDRA ACHARYA et al. 2006).

\subsubsection{Somatische Vorerkrankungen}

Die HRV ist erniedrigt bei Hypertonus (HTN) mit linksventrikulärer Hypertrophie, nach Herzinfarkt (MI) (RAJENDRA ACHARYA et al. 2006) und bei Herzinsuffizienzpatienten (HI), für die Letztgenannten teilweise so stark wie nach Herztransplantation (SZTAJZEL 2004). Für Diabetiker (DM) wird eine erniedrigte HRV als frühes Zeichen einer Neuropathie gewertet (RAJENDRA ACHARYA et al. 2006). Für das metabolische Syndrom ist ein Zusammenhang mit erhöhtem Sympathikus und erniedrigter vagaler HRV beobachtet worden (TENTOLOURIS et al. 2008). Dieser Zusammenhang ist auch schon bei jungen Männern mit erhöhtem BMI zu beobachten(SCHMID et al. 2010). 


\subsubsection{Psychische Vorerkrankungen}

Bei psychischen Vorerkrankungen sind verschiedene Veränderungen der HR und der HRVParameter beschrieben worden.

Für Depression sind die Ergebnisse uneinheitlich (ROTTENBERG 2007). Die HR wird teilweise als erhöht beschrieben (ROTTENBERG 2007). Für die HRV werden teilweise erniedrigte Werte für Depressive im Vergleich zu Nicht-Depressiven beschrieben (KEMP et al. 2010; RAJENDRA ACHARYA et al. 2006; MUECK-WEYMANN et al. 2002; CARNEY et al. 2001), teilweise so stark wie nach Herztransplantation (NAHSHONI et al. 2004). Eine erhöhte HR und erniedrigte HRV zeigten sich auch im Tierversuch (GRIPPO et al. 2002). Teilweise wird kein Unterschied bezüglich der HRV zwischen körperlich gesunden Depressiven und Nicht-Depressiven beschrieben (EINVIK et al. 2011; SAYAR et al. 2002). Als Ursache der uneinheitlichen Studienergebnisse wird unter anderem die Heterogenität der Symptomatik, die unter dem Begriff „Depression“ zusammengefasst wird, angesehen (ROTTENBERG 2007).

Angstpatienten zeigten in einigen Studien eine erhöhte HR und erniedrigte HRV-Parameter (FRIEDMAN 2007; ROTTENBERG 2007). Teilweise werden für Angstpatienten keine Unterschiede bezüglich der HRV zur Normalbevölkerung beschrieben (EINVIK et al. 2011). Anzumerken bleibt, dass viele der Studien Angstpatienten unter experimentellen Bedingungen im Stresstest messen (FRIEDMAN 2007). Dies kann die unterschiedlichen Ergebnisse erklären.

$\mathrm{Zu}$ VE und HRV konnte lediglich eine Arbeit (Stand 15.08.2011) in einer Pubmed-Recherche mit den Suchbegriffen „,vital exhaustion AND heart rate variability“ gefunden werden. Es zeigte sich eine erniedrigte HF für vital erschöpfte männliche Arbeiter (WATANABE et al. 2002).

Steigende Werte der Lebensqualität, insbesondere bei den körperlichen Aspekten der Lebensqualität, zeigten einen Zusammenhang mit erhöhten HRV-Parametern (VANGESTEL et al. 2011; HATHAWAY et al. 2000). Weitere aussagekräftige Arbeiten konnten in einer PubmedRecherche mit den Suchbegriffen „quality of life AND heart rate variability“ nicht gefunden werden (Stand 15.08.2011). 
Sowohl aufgrund der widersprüchlichen als auch der teilweise noch fehlenden Daten zeigt sich ein klarer Forschungsbedarf bezüglich des Zusammenhangs der genannten psychischen Variablen und der HR beziehungsweise der HRV-Parameter.

\subsection{Kardiale Schweregradmarker und ihr Zusammenhang mit HRV und Psyche}

Neben der Untersuchung der Herzfrequenzvariabilität in Verbindung mit den psychischen Variablen wurden einige der in der Studie erhobenen somatischen Parameter in Zusammenhang mit der Herzfrequenzvariabilität und den psychischen Variablen gesetzt, um das Ausmaß der somatischen Schwere der Erkrankung als Confounder beziehungsweise Mediator beschreiben zu können. Besonderes Augenmerk lag dabei auf dem kardialen Peptid NT-proBNP, aber auch echokardiographische Parameter und die Gehstrecke im 6-Minuten-Gehtest wurden näher untersucht.

\subsubsection{NT-pro-BNP}

Das amino-terminale Fragment des Prohormons von BNP (NT-pro-BNP) ist eines der natriuretischen Peptide (NP). Diese Peptide werden insbesondere bei Volumenbelastung des Herzens ausgeschüttet. Sie können durch ihre hormonellen Eigenschaften die Herzinsuffizienz in einem kompensierten Stadium halten und die Entwicklung einer symptomatischen Herzinsuffizienz verzögern. Zu betonten ist dabei, dass die NP die parasympathische Signalweiterleitung unterstützen und eine Bradykardie auslösen (HERRING et al. 2001).

Das NT-pro-BNP zeigt keine starken Schwankungen nach Tageszeit oder bei Belastungen (MAIR et al. 2001). Für Frauen werden leicht höhere Werte beschrieben (MAIR et al. 2001). Die Plasmaspiegel der unterschiedlichen natriuretischen Peptide korrelieren stark miteinander (MUELLER et al. 2004; MAIR et al. 2001). Neben den diagnostischen Eigenschaften haben erhöhte NT-pro-BNP-Werte auch starke prognostische Aussagekraft bezüglich Gesamtmortalität, kardiovaskulärer Morbidität und Mortalität. Vergleichswerte sind laborspezifisch (MAIR et al. 2001). Insbesondere die Kombination von Depression und erhöhten BNP-Werten ist mit einer deutlich verschlechterten Prognose verbunden (PARISSIS et al. 2008).

Ein Zusammenhang mit psychischen Faktoren konnte für das amino-terminale Fragment des Prohormons von ANP (NT-pro-ANP) und NT-pro-BNP gezeigt werden: 
Für depressive Symptome, gemessen anhand der Hospital Anxiety and Depression Scale (HADS), konnte ein Zusammenhang mit erhöhten Werten für das NT-pro-ANP (HERRMANN-LINGEN et al. 2003) und für das NT-pro-BNP (BUNEVICIUS et al. 2006) nachgewiesen werden.

Für Angst zeigte sich für NT-pro-ANP eine inverse Korrelation bei Herzinsuffizienzpatienten (HERRMANN-LINGEN et al. 2003). Dieser Effekt könnte durch anxiolytische Eigenschaften erklärt werden, die den natriuretischen Peptiden in Studien nachgewiesen werden konnten (HERRMANN-LINGEN et al. 2003; STRÖHLE et al. 2001; BIRO et al. 1996). Auch für NTpro-BNP ergab sich, zumindest für männliche Patienten mit subklinischer ventrikulärer Dysfunktion, ein inverser Zusammenhang zwischen NT-pro-BNP-Plasmaspiegeln und niedrigen Werten für Angst in der HADS (HERRMANN-LINGEN et al. 2004).

Vitale Erschöpfung zeigte eine positive Korrelation mit NT-pro-ANP (HERRMANNLINGEN et al. 2003).

Niedrige subjektive Lebensqualität bei kardial vorerkrankten Probanden war mit erhöhten Werten des NT-pro-BNP (HERRMANN-LINGEN et al. 2004) und des NT-pro-ANP (HERRMANN-LINGEN et al. 2003) verbunden.

Zusammenfassend ist NT-pro-BNP ein wenig von äußeren Faktoren beeinflusster Parameter der Herzinsuffizienz. Für natriuretische Peptide werden teilweise Zusammenhänge mit psychischen Auffälligkeiten beschrieben.

\subsubsection{Echokardiographie}

Das Hauptinstrument, um systolische (definiert anhand der linksventrikulären Ejektionsfraktion (LVEF)) und diastolische Dysfunktion zu beschreiben, ist die Echokardiographie (ANGERMANN und ERTL 2004; REMME und SWEDBERG 2001). Die diastolische Dysfunktion wird definiert und quantifiziert unter anderem anhand des Blutflusses über der Mitralklappe (E/A-Quotient), des Pulmonalvenenflusses und des Gewebedopplers am Mitralring (PAULUS et al. 2007). Die Klassifikation der diastolischen Dysfunktion erfolgt anhand verschiedener Schemata (s. 6.1 Diastolischer Herzinsuffizienz Score im KNHI, S. 95).

Die LVEF zeigte bei kardiologischen Patienten (GORKIN et al. 1993), insbesondere auch bei Patienten mit Herzinsuffizienz (JUENGER et al. 2002), keinen Zusammenhang mit der Lebensqualität. Für Depression zeigte sich lediglich ein sehr schwacher Zusammenhang mit 
erniedrigter LVEF bei Herzinsuffizienzpatienten (ANGERMANN et al. 2011). Eine norwegische Studie zeigte eine Assoziation von weniger Depression und erniedrigten LVEF-Werten, aber keinen Zusammenhang zwischen LVEF und Angst (ULVIK et al. 2008). Damit sind die Zusammenhänge für Depression und LVEF widersprüchlich. In einer Pubmed-Recherche konnte für „vital exhaustion AND LVEF“ keine aussagekräftige Literatur gefunden werden (Stand 15.08.2011).

\subsubsection{6-Minuten-Gehtest}

Der 6-Minuten-Gehtest ist ein submaximaler alltagsähnlicher Belastungstest, der alternativ zur Spiroergometrie verwendet werden kann (HAASS et al. 2000). Auch für ältere Patienten ist dieser Test eine sichere Möglichkeit zur Objektivierung ihrer körperlichen Leistungsfähigkeit (ENRIGHT et al. 2003). Eine erniedrigte Gehstrecke stellt einen unabhängigen Prognosefaktor für die Mortalität dar (HAASS et al. 2000).

Depressive Symptomatik und erniedrigte Lebensqualität korrelieren mit kürzeren Gehteststrecken (LORD und MENZ 2002; GORKIN et al. 1993). Ebenso zeigen Studien einen Zusammenhang von Angst und kürzeren Gehstrecken (GIARDINO et al. 2010). Für ,vital exhaustion AND walk test" fanden sich in einer Pubmed-Recherche keine adäquaten Arbeiten (Stand 15.08.2011).

In der vorliegenden Arbeit wird neben dem Zusammenhang Psyche und HRV auch der Zusammenhang der vorgenannten kardialen Schweregradmarker mit Psyche und HRV untersucht. 


\subsection{Ziele und Hypothesen}

Wie in den vorangegangenen Kapiteln erläutert, sind die Ergebnisse bezüglich der Zusammenhänge der betrachteten psychischen Variablen und der Herzfrequenzvariabilität uneinheitlich. Die Inkonsistenz der Befunde könnte in den unterschiedlichen Messsituationen begründet sein. Um zu prüfen, ob in unterschiedlichen Aktivierungszuständen des autonomen Nervensystems die Herzfrequenzvariabilität besonders ausgeprägte Zusammenhänge mit der Psyche zeigt, wurde ein experimenteller Untersuchungsaufbau mit Aktivitäts- (Gehtest), Taktatmungs- und Ruhephase gewählt.

Vermutet wird, dass eine autonome Dysfunktion (hier im Sinne einer Dominanz des Sympathikus und einer Supprimierung des Parasympathikus) als potentielle Erklärung für die erhöhte Mortalität bei psychischer Auffälligkeit im vorliegenden Kollektiv nachzuweisen ist.

Als Confounder wurden einige somatische Parameter bezüglich ihres Zusammenhangs zu Psyche und Herzfrequenzvariabilität überprüft.

Im Einzelnen werden folgende Hypothesen geprüft:

1.) Depressive Symptome

A) gehen mit einem erhöhten Sympathikotonus und einem reduzierten Vagotonus einher, erkennbar an erhöhter HR und erniedrigten globalen und vagalen sowie erhöhten sympathischen HRV-Parametern.

B) Dieser Zusammenhang ist unabhängig vom kardialen Status.

C) Besonders ausgeprägt tritt er während der Taktatmung auf.

D) Die beschriebenen Effekte werden für Männer und für Frauen erwartet.

2.) Ebensolche Zusammenhänge, wie für Depressivität mit HR und HRV unter 1.) postuliert, werden auch für Angst, Vitale Erschöpfung und körperliche Lebensqualität erwartet. 
3.) Insbesondere die hochfrequenten, vagalen (rMSSD, pNN50, HF) HRV-Werte zeigen klare Zusammenhänge zu dem Ausmaß der kardialen Vorerkrankungen und den kardialen Schweregradmarkern. Bei Zeichen starker Vorerkrankung oder Schädigung wird eine niedrige HRV erwartet.

4.) Höhere HR und erniedrigte HRV in Ruhe sagen eine kürzere 6-Minuten-Gehstrecke voraus.

5.) Kardiale Schweregradmarker korrelieren schwach mit Depressivität, Vitaler Erschöpfung und der körperlichen Funktionsfähigkeit der Lebensqualität, aber nicht mit Angst.

Die Datenerhebung und Auswertung zur Prüfung der oben genannten Hypothesen wird im folgenden Kapitel erläutert. 


\section{Probanden und Methoden}

Dieses Kapitel beinhaltet die Erläuterungen zu Untersuchungsmethoden und Probanden. Sofern nicht eindeutig nur weibliche Personen gemeint waren, wurde zur Vereinfachung die männliche Form benutzt.

\subsection{Kompetenznetz Herzinsuffizienz}

\subsubsection{Projektbeschreibung}

Das Kompetenznetz Herzinsuffizienz (KNHI) ist ein deutschlandweites Netzwerk, im Jahre 2003 vom Bundesministerium für Bildung und Forschung mit dem Ziel gegründet, in einem interdisziplinären Bemühen die Inzidenz und Prävalenz der Herzinsuffizienz sowie deren Risikovorerkrankungen zu untersuchen, ihre Diagnostik und Therapie zu verbessern und zu klären, welche Rolle psychische und soziale Faktoren in diesem Zusammenhang spielen. Beteiligt sind Kardiologen, Allgemeinmediziner, Labormediziner, Psychosomatiker und Epidemiologen an verschiedenen Universitätskliniken, Forschungsinstituten, Herzzentren, HerzKreislauf-Kliniken, mehreren hundert Arztpraxen, mehreren Rehabilitationskliniken sowie weitere Organisationen und Verbände. Die Koordinationszentrale des Kompetenznetzes befindet sich an der Charité in Berlin. Aus der landesweiten und fächerübergreifenden Zusammenarbeit soll ein möglichst großer Informationsgewinn resultieren. Das KNHI befasst sich innerhalb mehrerer Teilprojekte mit verschiedenen Aspekten der Herzinsuffizienz. Die vorliegende Arbeit ist im Rahmen des Teilprojekts 7 zur diastolischen Herzinsuffizienz in der Universitätsmedizin Göttingen (UMG) entstanden. Die Untersuchungen wurden an einem asymptomatischen und symptomatischen Risikokollektiv für Herzinsuffizienz durchgeführt. Studienbeginn des Teilprojekts 7 war am 5.07.2004 (detailliertere Informationen in DIETZ 2004).

Ein endgültiger positiver Beschluss der Ethikkommission vom 05.07.2004 unter der Antragsnummer 22/2/04 liegt vor.

Die Untersuchungen zur vorliegenden Teilstudie fanden vom 21.01.2005 bis zum 19.06.2006 statt. Eingeschlossen wurden die Probanden während der Baseline aber zum Teil auch zum ersten Follow-up im Teilprojekt 7 in Göttingen. 


\subsubsection{Probandenrekrutierung}

Die Rekrutierung der Probanden erfolgte durch die Abteilung Allgemeinmedizin bei niedergelassenen Fachärzten für Allgemeinmedizin und hausärztlich tätigen Internisten in der Region Göttingen. In den Praxen wurden geeignete Probanden mit Hilfe der praxiseigenen Software anhand der kodierten Diagnosen identifiziert (nähere Informationen in BREMECKER 2010).

Die potentiellen Probanden des Risikokollektivs für Herzinsuffizienz beziehungsweise bekannte Herzinsuffizienzpatienten wurden über den Hausarzt mittels eines Anschreibens des KNHI-Projekts kontaktiert. Bei Interesse wurde über den Hausarzt ein Formular an die allgemeinmedizinische Abteilung des UMG geschickt. Von dieser Abteilung wurde die Terminvergabe durchgeführt. Für das erste Follow-up wurden die Probanden von verschiedenen Mitarbeitern des Kompetenznetzes telefonisch kontaktiert.

\subsubsection{Einschlusskriterien $\mathrm{KNHI}$}

Vorliegen von:

- arterieller Hypertonie(HTN) und/ oder

- Diabetes mellitus(DM) und/ oder

- Schlafapnoesyndrom und/ oder

- arteriosklerotischer Grundkrankheit und/ oder

- Herzinsuffizienz(HI)

- Alter 50-85 Jahre

- Schriftlichem Einverständnis des Probanden.

\subsubsection{Ausschlusskriterien}

Allgemein:

- mangelnde Kooperationsbereitschaft

- unzureichende Kommunikationsfähigkeit (geringe Deutschkenntnisse, Aphasien)

- Erkrankungen, die die Einwilligungsfähigkeit einschränken

- geographische Gründe, die eine Studienteilnahme unmöglich machen. 
Speziell für die vorliegende Teilstudie:

- Schrittmacherpatienten

- Zustand nach Herztransplantation

- körperliche, nicht kardial bedingte Unfähigkeit zum 6-Minuten-Gehtest

- ausgeprägte Herzrhythmusstörungen in Anamnese oder RuheElektrokardiographie (EKG) (z. B. Vorhofflimmern, Bigeminus)

- Sicherheitsbedenken aufgrund akuter gesundheitlicher Probleme (stark erhöhter Blutdruck, aktuelle Angina pectoris oder Dyspnoe) vor Beginn des Gehtests

- psychologischer Fragebogen nicht, beziehungsweise mehr als 14 Tage vor der Langzeit-EKG-Untersuchung ausgefüllt.

\subsubsection{Aufklärung}

Die Probanden wurden durch die Mitarbeiter der Kardiologie ausführlich über die Studie informiert. Nach Aufklärung und schriftlicher Einwilligung zur Studienteilnahme begannen die Untersuchungen. Vor der LZ-EKG-Untersuchung wurden die Probanden erneut aufgeklärt und erteilten mündlich erneut ihre Einwilligung.

\subsubsection{Untersuchungsaufbau}

Die Probanden wurden in der kardiologischen Abteilung des UMG vorstellig und von den für das KNHI-Projekt forschenden Doktoranden der Kardiologie sowie den zuständigen kardiologischen Assistenzärzten untersucht. Die Untersuchung bestand, nach Aufklärung und Einwilligung, aus Anamnese, klinischer Untersuchung mit Erfassung der Vitalparameter, RuheEKG, Blutentnahme und echokardiographischer Untersuchung. Das Ruhe-EKG wurde im Anschluss an die körperliche Untersuchung durchgeführt. Die Blutentnahme erfolgte nach einer Liegephase direkt im Anschluss an die EKG-Ableitung. Gelegentlich wurde diese Blutentnahme nach dem Gehtest, aber wiederum erst nach einer Liegephase, durchgeführt. Im Rahmen der Erstuntersuchung erfolgte eine echokardiographische Untersuchung auf das Vorliegen einer systolischen beziehungsweise diastolischen Herzinsuffizienz (s. 6.1 Diastolischer Herzinsuffizienzscore im KNHI, S.95). Diese wurde vor oder nach dem 6-Minuten-Gehtest durchgeführt. Nähere Informationen sowie Quellen zu der genauen Vorgehensweise bei der kardiologischen, laborchemischen, Ruhe-EKG- und echokardiographischen Untersuchung finden sich in der kardiologischen Promotion desselben Projekts (BREMECKER 2010). 
Unter Beachtung der Ausschlusskriterien wurden Probanden in die LZ-EKG-Untersuchung eingeschlossen, sofern eine Studienmitarbeiterin zur Verfügung stand und die Probanden der freiwilligen Teilnahme zustimmten. Die Untersuchung erfolgte unter Einschluss des 6Minuten-Gehtests meist zwischen körperlicher und echokardiographischer Untersuchung. Selten wurden Probanden an einem späteren Tag für die LZ-EKG-Untersuchung einbestellt. Dies erfolgte maximal 14 Tage nach der Erstuntersuchung. Für diesen Zeitraum wird eine Aktualität der erhobenen Werte weiterhin angenommen. Der KNHI-Fragebogen mit verschiedenen psychologischen Fragebögen wurde im Rahmen des Erstkontaktes von den kardiologischen Mitarbeitern ausgehändigt und kurz erläutert. Weitere Informationen zu den Fragebögen befanden sich auf dem Deckblatt des Fragebogens (s. 6.2 Fragebogen KNHI mit Polung, ab S.96).

Eine Repräsentativitätsprüfung des untersuchten Kollektivs findet sich unter 2.5.3.1 Repräsentativitätsprüfung (S.34).

\subsection{Langzeit-EKG-Untersuchung}

Die LZ-EKG-Untersuchungen wurden größtenteils von Vera C. Duden, aber auch von einer weiteren Doktorandin und einer MTA durchgeführt. Die Auswertung der EKGs erfolgte ausschließlich durch Vera C. Duden.

\subsubsection{Versuchsplanung LZ-EKG}

Die Teil-Stichprobe des KNHI-Kollektivs wurde mittels Langzeit-EKG in einem dreistufigen experimentellen Setting untersucht. Die Untersuchungen fanden zwischen $8 \mathrm{Uhr}$ und $20 \mathrm{Uhr}$ statt. Die erste Phase des LZ-EKGs war der 6-Minuten-Gehtest (Geh). Nach mindestens fünf Minuten Ruhephase, frühestens aber, wenn der Proband eupnoeisch, objektiv wie subjektiv beschwerdefrei war, begann die zweite Phase der Untersuchung. Die zweite Untersuchungsphase bestand aus einer fünfminütigen Taktatmungsphase (TA) im Sitzen. Direkt im Anschluss an die Taktatmungsphase folgte eine zweite Ruhephase (RUH) von fünf Minuten, während derer die Probanden angewiesen waren, nicht zu reden und ruhig sitzen zu bleiben. Auffälligkeiten während der Untersuchung und die erhobene Gehstrecke des 6-MinutenGehtests wurden dokumentiert. 


\subsubsection{6-Minuten-Gehtest}

Der 6-Minuten-Gehtest wurde im Rahmen der vorliegenden Auswertung als Belastungsphase zur Stimulation des Sympathikus genutzt. Der Proband wurde aufgefordert, innerhalb von sechs Minuten, so schnell wie für ihn bei zügigem Gehen machbar, so viel Strecke wie möglich zurückzulegen. Die Messstrecke war eine ebene Strecke von 100 Metern im Inneren des UMG, auf der die Probanden auf- und abschritten. Nach jeweils 100 Metern musste gewendet werden. Pausen während des Gehens aufgrund von z. B. Schmerzen in den Beinen waren explizit erlaubt. Bei den Pausen lief die Zeit weiter. Der Proband war angehalten, nach Abklingen der akuten Beschwerden den Test fortzusetzen. Die Länge der Pausen wurde dokumentiert. Ebenso wurden klinische Symptome wie Brustenge, Atemnot, Schwindel, Schmerzen und andere Symptome erfragt und dokumentiert. Ein vorzeitiges Abbrechen wurde mit der Zeit, nach der der Gehtest abgebrochen wurde, und unter den Stoppkriterien ,allgemeine Erschöpfung“, „Angina pectoris“, „Claudicatio“, „Dyspnoe“ oder „andere“ dokumentiert. Im Rahmen der kardiologischen Untersuchung wurden Blutdruck und Herzfrequenz gemessen, um Probanden, die die Untersuchung gefährden würde, zu identifizieren. Probanden, die auffällige Parameter zeigten, zum Beispiel Blutdruck systolisch über $200 \mathrm{mmHg}$, wurden von der LZ-EKG-Untersuchung ausgeschlossen. Dieses Vorgehen entspricht den Empfehlungen in HAASS et al. (2000). Falls es während des Gehtests zu Angina pectoris (8 Fälle), Atemnot (meist nur leichte Dyspnoe: 17 Fälle) oder anderen Beschwerden kam, wurde, sofern die Symptomatik in Situation und Schwere undiagnostiziert und unbehandelt war, der für die Studie zuständige Kardiologe verständigt (7 Fälle), teilweise mit dem Hausarzt Rücksprache gehalten und/oder der Patient angewiesen, seinen Hausarzt zu informieren ( 3 der 7 Fälle). In keinem Fall war eine Einleitung notfallmäßiger Therapie notwendig. In der Regel war die Symptomatik bekannt und vom Probanden selbst einschätz- und beherrschbar.

\subsubsection{Taktatmung}

Während der Taktatmung kommt es zu dem in der Einleitung beschriebenen Phänomen (s.1.3.3.2 Atmung, S.10). Der Proband war während der Taktatmung angewiesen, über vier Sekunden lang einzuatmen und über sechs Sekunden auszuatmen, entsprechend 6 Atemzügen pro Minute. Die Phasen wurden mit einer Stoppuhr gemessen und die Phasenwechsel vom Untersucher angezeigt. Zum Teil wurde zur Motivation mitgeatmet. Gelang es dem Probanden nicht, den Atemrhythmus einzuhalten, wurde er aufgefordert, möglichst langsam zu atmen. Kreislaufschwierigkeiten während der Taktatmungsphase wurden dokumentiert und in einem Fall durch Hinlegen behoben. 


\subsubsection{LZ-EKG-Untersuchung}

Die Probanden wurden mit einem digitalen Langzeit-EKG-Gerät Medilog AR 12 der Firma Oxford Instruments untersucht. Dieses Gerät bietet ein 3-Kanal-EKG. Verbunden war das Gerät mit dem Probanden über ein 5-lead-Kabel der Firma Huntleigh Healthcare in Cardiff, UK. Im Medilog IR Monitor der Simple View Version 2.2 Release 11 der Firma Oxford Instruments Medical wurde die Aufnahmequalität eingestellt (s. Tab. 1). Wie von verschiedenen Fachgruppen empfohlen, hatten die Aufnahmequalitäten eine Digitalisierungsrate von $256 \mathrm{~Hz}$ ( $>250 \mathrm{~Hz}$ in ACC/AHA guidelines for ambulatory electrocardiography: CRAWFORD et al. 1999) und mindestens 10 BIT (BIT= „,binary digit“, $n$ BIT entspricht $2^{\mathrm{n}}$ ) (TASK FORCE OF THE EUROPEAN SOCIETY OF CARDIOLOGY AND THE NORTH AMERICAN SOCIETY OF PACING AND ELECTROPHYSIOLOGY 1996; MEDILOG AR 12 PRIMER 2002).

\begin{tabular}{lcccc}
\hline & $\begin{array}{c}\text { fix sample rate } \\
\text { (Abtastfrequenz) }\end{array}$ & $\begin{array}{c}\text { low pass input filter } \\
\text { frequency }\end{array}$ & \multicolumn{2}{c}{$\begin{array}{c}\text { Recording profile } \\
\text { Double Time/ } \\
\text { High Density }\end{array}$} \\
\hline Channel 1 & $4.096 \mathrm{~Hz}$ & $1.900 \mathrm{~Hz}$ & $256 \mathrm{~Hz}$ & $12 / 16 \mathrm{BIT}$ \\
\hline Channel 2 & $512 \mathrm{~Hz}$ & $230 \mathrm{~Hz}$ & $256 \mathrm{~Hz}$ & $10 / 16 \mathrm{BIT}$ \\
\hline Channel 3 & $512 \mathrm{~Hz}$ & $230 \mathrm{~Hz}$ & $256 \mathrm{~Hz}$ & $10 / 16 \mathrm{BIT}$
\end{tabular}

Tabelle 1 Einstellungen der Aufnahmequalität des Langzeit-EKG-Geräts der Firma Medilog Typ AR12 (Medilog AR 12 Primer 2002)

\subsection{Psychologische Daten}

In einem ausführlichen Fragebogen wurden Daten $\mathrm{zu}$ unterschiedlichen psychologischen Konzepten sowie soziodemographische Daten erhoben. In der Regel wurde der Fragebogen während des Aufenthalts im UMG ausgefüllt. Selten wurde er den Probanden mit nach Hause gegeben und von ihnen zurückgesandt (s. 6.2 Fragebogen KNHI mit Polung, ab S.96). Für diese Teilstudie wurden die Hospital Anxiety and Depression Scale (HADS), der MaastrichtFragebogen (MQ) und die Skala der körperlichen Funktionsfähigkeit des Short Form Health Survey (SF-36) näher betrachtet. 


\subsubsection{Hospital Anxiety and Depression Scale}

Die Hospital Anxiety and Depression Scale (HADS) wurde von ZIGMOND und SNAITH 1983 veröffentlicht. Die HADS ist ein Selbstbeurteilungsfragebogen zum Screening von somatisch kranken Patienten auf die beiden häufigsten psychiatrischen Erkrankungen, Angst und Depression. In dieser Studie wurde die deutsche Version (HADS-D) verwendet. Diese wurde 1994 von HERRMANN und BUSS vorgestellt (HERRMANN und BUSS 1994).

Die HADS hat 14 Items aufgeteilt in zwei Subskalen mit jeweils sieben Items (HERRMANN und BUSS 1994). Die Subskala Angst (HADS-D/A) erfasst eine „generalisierte, frei flottierende Angstsymptomatik“ (HERRMANN-LINGEN et al. 2005). Die Subskala Depressivität (HADS-D/D) erfasst eine milde Depressivität, eine sogenannte ,endogenomorphe“ Symptomatik (KLEIN 1974; SNAITH 1987). Jedes Item hat vier Antwortmöglichkeiten mit Punktewerten von 0-3 (HERRMANN-LINGEN et al. 2005).

Die Auswertung erfolgt durch Addition der Punktwerte zunächst in den Subskalen. In dieser Studie wird die Subskala Depressivität mit Werten über 8 als auffällig (positiv) gewertet, die Angstskala mit Werten über 10, wie im Handbuch empfohlen. Die Objektivität des Fragebogens ist durch schriftliche Instruktionen und standardisierte Auswertungsrichtlinien gewährleistet (HERRMANN-LINGEN et al. 2005). Ist der Wert der Subskala auffällig, so wird es in dieser Arbeit als „Depressivität/ Angst vorhanden“ beschrieben.

Die Reliabilität, gemessen anhand der folgenden Kennwerte, zeigt insgesamt eine befriedigende und homogene Teststruktur. Die interne Konsistenz, gemessen mit Cronbach's AlphaKoeffizient, ist als gut zu bezeichnen. Sie beträgt für die Angstskala (HADS-D/A) 0,8 und für die Depressivitätsskala (HADS-D/D) 0,81. Die Trennschärfe (korrigierte Item-TotalKorrelation) liegt zwischen 0,49 (minimal) und 0,72 (maximal) mit mittlerer Trennschärfe bei 0,61. Der Split-half-Koeffizient nach Spearman-Brown liegt für beide Skalen bei 0,81, für die Gesamt-HADS-D bei 0,88. Die Retest-Reliabilität, angegeben mit dem Stabilitätskoeffizienten nach Pearson $\left(\mathrm{r}_{\mathrm{tt}}\right)$ liegt global für beide Subskalen bei 0,71. In einem Intervall von 2 Wochen liegt sie zwischen 0,81 und 0,89 (HERRMANN-LINGEN et al. 2005).

Die Validität der HADS-D wurde mit Hilfe der im Folgenden aufgeführten Parameter festgestellt. Die konvergente Validität zeigte im Vergleich der HADS-D-Angstskala mit der Angstskala des State-Trait-Angstinventars von LAUX et al. (1981) Werte um 0,68. Die Depressivitätsskala des HADS wurde mit der Depressivitätsskala der Paranoid-DepressivitätsSkala von ZERSSEN und KOELLER (1976) verglichen. Dabei wurden an verschiedenen Kollektiven Werte von 0,54 (Studenten) und 0,76 (Psychiatriepatienten) gemessen. Die Fakto- 
renanalyse, als Zeichen der Konstruktvalidität, zeigte für die HADS-D eine zweifaktorielle Skalenstruktur, wobei der erste Faktor hoch mit der Depressivitätsskala $(r=0,92)$ und der zweite hoch mit der Angstskala korreliert ( $\mathrm{r}=0,93)$. Im Rahmen der diskriminativen Validität wurde die Interkorrelation der beiden Subskalen berechnet, wobei sich erhebliche Interkorrelationen fanden. Dies könnte die Komorbidität von Angst- und Depressivitätssymptomen illustrieren. Sensitivität und Spezifität lässt sich nicht eindeutig beschreiben, da es keine klaren Cut-off-Werte gibt. In den meisten Studien lagen die Werte für Sensitivität und Spezifität über $80 \%$. Dies zeigt, dass die HADS psychische Symptomatik erkennt, allerdings sind die Werte aufgrund der unterschiedlichen Cut-off-Werte und mangelnder Dokumentation nicht diagnoseweisend (HERRMANN-LINGEN et al. 2005).

Für ihre eigentliche Aufgabe in der Erkennung von Angst und Depressivität bei somatischen Probanden ist die HADS allerdings besser als nicht psychiatrisch oder psychosomatisch spezialisierte Mediziner (HERRMANN 1997). Insgesamt zeigt die HADS-D ausreichende instrumentelle Eigenschaften (HERRMANN und BUSS 1994). Im Vergleich zur Originalversion, soweit die Daten zum Vergleich vorliegen, scheint die HADS-D der HADS gleichwertig zu sein (HERRMANN 1997).

\subsubsection{Maastricht-Fragebogen}

Der Maastricht-Fragebogen (MQ) ist ein Selbstbeurteilungsfragebogen zur Messung der „Vitalen Erschöpfung“, der im Rahmen der Rotterdam-Civil-Servants-Studie entwickelt wurde (APPELS et al. 1987).

Er wurde zur Erforschung eines Gefühls der Erschöpfung vor einem Herzinfarkt, das in kardiologischen Studien häufig als Prodromalsyndrom beschrieben wurde, entwickelt. Patienten beschreiben den Beginn dieses Symptoms etwa 12-18 Monate vor einem MI oder plötzlichen Herztod. Eine Vitale Erschöpfung allein scheint keine Ursache für einen Herzinfarkt zu sein (APPELS et al. 1987).

Vitale Erschöpfung ist ein der Depression ähnliches Konstrukt. Definiert wurde sie als „ein Zustand, der vorhanden ist, wenn ein Individuum nicht nur über unübliche Müdigkeit und abnehmende Energie klagt, sondern auch über ein Gefühl, abgelehnt und besiegt worden zu sein. Sich nach dem Aufstehen erschöpft zu fühlen, ist hoch charakteristisch für diesen $\mathrm{Zu}-$ stand. Vitale Erschöpfung wird häufig mit einer zunehmenden Irritabilität und einem Verlust der Libido assoziiert. Symptome von Depression können mit Vitaler Erschöpfung assoziiert sein, aber nicht notwendigerweise. Für gewöhnlich ist das Selbstwerterleben nicht reduziert 
und Schuldgefühle fehlen“ (APPELS et al. 1987, S.21-22, Originalzitat übersetzt von Vera C. Duden).

Für die Entwicklung des MQ wurden 37 Items aus einem Fragebogen der ImminentMyocardial-Infarction-Rotterdam-Studie, deren Fähigkeit zur Diskriminierung zwischen Probanden, denen eine Koronarerkrankung drohte, und einem Normalkollektiv bereits bekannt war, mit 21 weiteren Items aus klinischen Interviews kombiniert. Aus diesem Gesamtpool von 58 Fragen wurde im Rahmen der Studie von APPELS et al. der MQ von 21 Items entwickelt. Ausgewählt wurden die Items des endgültigen Fragebogens nach ihrer Fähigkeit, „harte" Koronarereignisse (wahrscheinlicher oder gesicherter Herzinfarkt oder kardialer Tod) vorherzusagen. 24 der 58 Fragen waren in der Lage, ein „hartes“ Koronarereignis vorherzusagen. 16 entstammten dem Fragebogen der Imminent-Myocardial-Infarction-Rotterdam-Studie, 8 waren neue Items. Zwei Fragen mussten aus dem endgültigen Fragebogen entfernt werden, weil ihre Vorhersagekraft nicht sicher war. Eine Frage wurde entfernt, weil sie in der Konzeptvalidität nicht als sicher eingestuft wurde. Insgesamt sind im endgültigen MaastrichtFragebogen 21 Items auf einer Skala vorhanden (APPELS et al. 1987).

In dieser Arbeit wurde eine deutsche Übersetzung von Prof. Dr. med C. Herrmann-Lingen verwendet (schon verwendet in HERRMANN-LINGEN et al. 2003).

Die 21 Items können mit „Ja“, „Nein“ oder „?“ beantwortet werden. Für jede positive Antwort gibt es zwei Punkte, ein ,,? “ bekommt einen Punkt, eine negative Antwort keinen Punkt. Bei den Fragen 9 und 14 ist die Punkteverteilung umgekehrt. Die Punkte werden addiert. Die Skala kann insgesamt Punktwerte zwischen 0 und 42 erreichen (APPELS et al. 1987). In dieser Arbeit wurden Werte über 19 als auffällig gewertet und dann als „Vitale Erschöpfung vorhanden" beschrieben.

Die Reliabilität, gemessen anhand der internen Konsistenz, war mit einem Cronbach's AlphaKoeffizienten von 0,89 hoch. Als Zeichen der konvergenten Validität wurde der MQ mit dem bereits als prädiktiv für Herzinfarkt etablierten 37-Item-Fragebogen der ImminentMyocardial-Infarction-Rotterdam-Studie korreliert. Die Korrelation lag bei 0,92, wobei zu bedenken bleibt, dass 16 der Fragen identisch sind (APPELS et al. 1987).

\subsubsection{Short Form Health Survey}

Der Short Form Health Survey mit 36 Fragen (SF-36) ist die Kurzform des ursprünglich in der Medical Outcome Study eingeführten Health Surveys (TARLOV et al. 1989). Die gekürzte Form wurde von WARE und SHERBOURNE 1992 vorgestellt. Das dem SF-36 zugrunde liegende Konzept der Lebensqualität beschreibt ein subjektives Empfinden der Gesundheit 
(BULLINGER 1997). Lebensqualität wird in der Regel als multidimensionales Konzept angesehen, wobei unter anderem psychisches Befinden, körperliche Verfassung, funktionale Kompetenz und soziale Beziehungen berücksichtig werden (BULLINGER und KIRCHBERGER 1998). Der SF-36 erfasst die gesundheitsbezogene Lebensqualität der Probanden durch einen verhaltensnahen Selbstbericht über soziale, psychische, körperliche und alltagsnahe Aspekte. Durch seine Ökonomie, leichte Handhabbarkeit und internationale Verbreitung hat er sich neben seinen zufrieden stellenden psychometrischen Testergebnissen als sinnvolles Messinstrument der Lebensqualität gezeigt (BULLINGER 2000). 1993 wurde die Originalform des SF-36 in zwei Schritten auf seine psychometrischen Merkmale getestet (McHORNEY et al. 1993 und 1994). Die deutsche Fassung wurde nach den Leitlinien des International-Quality-of-Life-Assessment-Projekts (AARONSON et al. 1992) von Monika Bullinger übersetzt (BULLINGER 1995).

Der SF-36 misst mit 36 Fragen in acht Subskalen (,körperliche Funktionsfähigkeit“ 10 Fragen, „körperliche Rollenfunktion“ 4 Fragen, „körperliche Schmerzen“ 2 Fragen, ,allgemeine Gesundheitswahrnehmung“ 5 Fragen, „Vitalität“ 4 Fragen, „,soziale Funktionsfähigkeit“ 2 Fragen, „emotionale Rollenfunktion“ 3 Fragen, ,psychisches Wohlbefinden“ 5 Fragen) und zwei Summenwerten (körperliche und seelische Gesundheit) die Lebensqualität. Zusätzlich gibt es eine Frage zum Vergleich des aktuellen Gesundheitszustands mit dem im vorangegangenen Jahr. Die Items sind binär skaliert (ja/nein) oder haben bis zu sechsstufige Antwortskalen (BULLINGER 2000).

Die Auswertung erfolgt nach einem vorgegebenen computerisierten Algorithmus, wobei teilweise gewichtete Items summiert und anschließend transformiert werden (BULLINGER UND KIRCHBERGER 1998).

Der deutsche SF-36 wurde an verschiedenen Probandengruppen auf seine psychometrischen Eigenschaften getestet (BULLINGER et al. 1995).

In dieser Arbeit wird nur die Skala „körperliche Funktionsfähigkeit“ als objektiver Marker für subjektives Gesundheitsempfinden und subjektive Lebensqualität verwendet. Die Reliabilität der Skala der körperlichen Funktionsfähigkeit war mit einem Cronbach's Alpha von 0,94 in der Normstichprobe hoch. Bezüglich der Validität konnte Folgendes festgestellt werden: die konvergente Validität mit dem Nottingham Health Profile ergab eine genügend hohe Korrelation auch für die Skala körperliche Funktionsfähigkeit. Die diskriminante Validität war in allen Populationen zufrieden stellend (BULLINGER UND KIRCHBERGER 1998).

Um auch für die körperliche Funktionsfähigkeit eine Bewertung nach schlechter beziehungsweise guter körperlicher Funktionsfähigkeit zu ermöglichen, wurde das Kollektiv dichotomi- 
siert. Da in der vorliegenden Stichprobe 13-21\% der Probanden für Depressivität, Angst und Vitale Erschöpfung auffällig waren, wurde eine vergleichbare Gruppengröße angestrebt, die bei einem cut-off Wert von 60 Punkten in der stetigen Skala der körperlichen Funktionsfähigkeit erreicht werden konnte. Damit waren $18 \%$ der Probanden in der Gruppe für niedrige körperliche Funktionsfähigkeit. Einen offiziellen cut-off-Wert gibt es für den SF-36 nicht.

\subsection{Dateneingabe}

Die Daten wurden mittels interner Codenummer pseudonymisiert erhoben und aufbewahrt. Die kardiologischen Daten, sowie Labordiagnostik und apparative Diagnostik wurden von den kardiologischen Doktoranden erhoben und eingegeben.

Die Fragebogendaten wurden von Vera C. Duden und zwei weiteren psychosomatischen Doktorandinnen eingegeben. Bei der Eingabe der Fragebögen wurden doppelt angekreuzte Werte, sofern sie direkt benachbart waren, gemäß der Polung (s.6.2 ab S.96) in Richtung höherer Belastung gewertet. Lagen eine oder mehrere weitere Antwortmöglichkeiten zwischen den angekreuzten Optionen, wurde die Antwort als fehlend gewertet. Ebenso, wenn bei lediglich zwei Antwortmöglichkeiten beide angekreuzt waren.

Die oben genannten Daten der Studie wurden zentral in einer Datenbank des Leipziger Koordinierungszentrums für klinische Studien (KKSL) gesammelt. Die Eingabe erfolgte über eData Entry online. Durch das Koordinierungszentrum erfolgte nach Datenprüfung und QueryBearbeitung die Erstellung einer Statistikdatei.

Die in dieser Teilstudie erhobenen EKG-Daten wurden nach der Bearbeitung mit Medilog Darwin Version V.1.5.11 zunächst manuell in einer Exceldatei eingetragen und schließlich über die interne Codenummer mit den übrigen statistischen Daten zusammengeführt.

\subsection{Datenbearbeitung}

\subsubsection{Fragebogenscores}

Im Rahmen der Datenbearbeitung wurden mittels SPSS die Scores der Fragebögen anhand der in den Handbüchern und Anweisungen vorgegebenen Algorithmen ermittelt und in die Datendatei eingefügt. 


\subsubsection{LZ-EKG-Bearbeitung und -Auswertung}

Die Auswertung des 3-Kanal-LZ-EKGs wurde von Vera C. Duden durchgeführt. Verwendet wurde die Auswertungssoftware Medilog Darwin in der Version V.1.5.11 der Firma Oxford Systems. In der verwendeten Version wurde durch Thomas Niederl, Firma Oxford Systems, eine Funktion zur millisekunden-genauen Bestimmung des zu untersuchenden Zeitfensters eingefügt. Die Berechnungen erfolgten in den Standardeinstellungen der genannten Softwareversion.

Die Datenmenge wird mit verschiedenen Berechnungen dargestellt. Die Werte der Time Domain werden durch statistische Berechnungen anhand der Herzrate und der NN-Intervalle dargestellt. Die Parameter der Frequency Domain wurden von einem auto-regressiven Modell 50. Ordnung nach BURG berechnet (MEDILOG DARWIN HOLTER ANALYSIS 2006b). Die Berechnungen waren auf eine Blockgröße von 1 bezogen. Blockgröße ist die Anzahl der Schläge in einer Folge, deren Schläge vorher definierte Kriterien erfüllen müssen, um in die Berechnung einbezogen zu werden (MEDILOG DARWIN HOLTER ANALYSIS 2006b). Lineares Detrending war aktiviert. Die FFT-Breite, dies ist die Breite des betrachteten Abschnitts der Messung für die Frequency-Berechnung, betrug 64. Dies verringert die Varianz und führt zu stabileren Ergebnissen (NIEDERL 2008). Die Frequency-Parameter wurden in $\mathrm{ms}^{2}$ angegeben. In Medilog-Darwin wurde nach Hochladen der EKG-Datei im Unterprogramm „Schlagformenanalyse“ entsprechend der Empfehlung der TASK FORCE OF THE EUROPEAN SOCIETY OF CARDIOLOGY AND THE NORTH AMERICAN SOCIETY OF PACING AND ELECTROPHYSIOLOGY 1996 eine visuelle Kontrolle aller drei EKGLinien auf Artefakte durchgeführt. Nach visueller Kontrolle wurden nur artefaktarme beziehungsweise artefaktfreie EKG-Zeilen zur Berechnung zugelassen. Generell wurden nur als Normalschlag definierte Schläge für die Berechnung verwendet. Zeigten alle 3 Kanäle Artefakte, wurden die Werte als fehlend für die untersuchte Phase gewertet. Nach der visuellen Kontrolle und eventuellem Ausschluss von EKG-Zeilen wurde das EKG von der Software analysiert. Bei Ausschluss einer oder mehrerer EKG-Zeilen wurde kritisch auf die maximalen beziehungsweise minimalen Herzfrequenzwerte beziehungsweise einen geringen Anteil an Normalschlägen $(\mathrm{ab}<95 \%)$ geachtet. War hier das EKG stark auffällig, wurde eine manuelle Schlag-für-Schlag-Kontrolle auf korrekte Definition als Normalschlag oder Extraschlag (z. B. ventrikuläre Extrasystole (VES)) durchgeführt. Bei dieser manuellen Kontrolle wurden fehlerhaft als Ventrikulärschlag erkannte Artefakte, fehlerhaft als Normalschlag erkannte Ventrikulärschläge und fehlerhaft als Ventrikulärschlag erkannte Normalschläge identifiziert und 
manuell korrigiert. Im Unterprogramm „Erweiterte Analyse“ wurden die einzelnen Phasen anhand der vorher platzierten „Patient Events“ manuell markiert. Die erste Phase (6-MinutenGehtest) von sechs Minuten wurde in drei Teilabschnitte mit jeweils 2 Minuten unterteilt, um durch Minimierung der natürlichen Herzfrequenztrends bei körperlicher Aktivität eine möglichst stabile Untersuchungsphase zu erzeugen. Nach Inspektion der Verteilung von SDNN und der Differenz von maximaler HR und minimaler HR zeigte sich nur ein minimaler Unterschied zwischen der mittleren und der letzten Phase von 2 Minuten. Aufgrund theoretischer Überlegungen wurde die letzte der drei zweiminütigen Phasen als stabilste Untersuchungsphase der Gehphase definiert. Daher wird diese Phase für den Vergleich mit den anderen Phasen herangezogen. Phase 2 (TA) und 3 (RU) wurden ebenfalls einzeln manuell markiert und als 5-Minuten-Fenster analysiert. Aus der Time-Domain-Analyse wurden die Werte mittlere HR, SDNN, rMSSD, pNN50, Gültig, aus der Frequenzanalyse die Werte LF, HF, Total, Log LF/HF (entspricht im deutschen Sprachraum Ln LF/HF) für die weitere Untersuchung verwendet (s. 6.3 Parameter HRV auch für Erläuterung der Abkürzungen, ab S. 110).

\subsubsection{Rhythmusausschlüsse}

Da insbesondere bei kurzen Messungen die EKGs frei von Arrhythmien sein sollten (TASK FORCE OF THE EUROPEAN SOCIETY OF CARDIOLOGY AND THE NORTH AMERICAN SOCIETY OF PACING AND ELECTROPHYSIOLOGY 1996 ), wurden, nach bereits erfolgtem Ausschluss aufgrund der visuellen Kontrolle, zusätzlich anhand der in Tabelle 2 aufgeführten Kriterien mutmaßlich rhythmisch auffällige EGKs aus der Untersuchung ausgeschlossen und als fehlend gewertet. Erfasst werden sollten Probanden mit absoluter Arrhythmie (AA), durchgehendem Bigeminus, Trigeminus oder gehäuften ventrikulären VES, die weder in Anamnese noch im Ruhe-EKG erkennbar waren.

\begin{tabular}{cccc}
\hline & $\begin{array}{c}\text { Ausschluss V. a. AA (pNN50 \& } \\
\text { HR Differenz auffällig) }\end{array}$ & Ausschluss V.a. VES \\
\hline pNN50 & $\begin{array}{c}\text { HR Diff. (Max- } \\
\text { Min) }\end{array}$ & Gültig \\
\hline Gehtest & $>20 \%$ & $>50 \mathrm{Bpm}$ & $<80 \%$ \\
\hline Taktatmung & $>30 \%$ & $>30 \mathrm{Bpm}$ & $<80 \%$ \\
\hline Ruhephase & $>20 \%$ & $>20 \mathrm{Bpm}$ & $<80 \%$ \\
\hline
\end{tabular}

Tabelle 2 Ausschlusskriterien anhand EKG Parametern. „HR-Diff“ beschreibt die Differenz zwischen der höchsten und niedrigsten gemessenen Herzfrequenz. „Gültig“ war definiert als Anteil der Normalschläge an den gesamten untersuchten Herzschlägen. 


\subsubsection{Ausreißer}

Nach Plausibilitätsüberlegungen und Inspektion der Verteilung wurden folgende Parameter als Grenzwerte für Ausreißer festgelegt und Werte die jenseits dieser Grenzen lagen in der Datenbank als Missing gewertet (s. Tabelle 3). Im Sonderfall Ln LF/HF wurde immer dann der Parameter als Missing gewertet, wenn einer der beiden Bestandteile (LF oder HF) als Missing gewertet werden musste. Waren in einer LZ-EKG-Phase drei oder mehr Ausreißer vorhanden, wurde die gesamte Phase als Missing gewertet.

\begin{tabular}{|c|c|c|c|c|c|c|c|}
\hline Parameter & Min & Max & Ausreißer & $\begin{array}{l}\text { Ausreißer } \\
\text { Gehtest } \\
\text { (N) }\end{array}$ & $\begin{array}{l}\text { Ausreißer } \\
\text { Taktatmung } \\
\text { (N) }\end{array}$ & $\begin{array}{c}\text { Ausreißer } \\
\text { Ruhephase } \\
\text { (N) }\end{array}$ & $\mathbf{N}$ \\
\hline rMSSD & 0 & 200 & $>200$ & 1 & 2 & 2 & \\
\hline pNN50 & 0 & 0,5 & $>0,5$ & 4 & 10 & 11 & \\
\hline SDNN & 0 & $\begin{array}{c}\text { Ruhe- } \\
\text { /Gehphase } \\
100 \\
\text { Taktatmung } \\
120\end{array}$ & $\begin{array}{l}>100 \\
>120\end{array}$ & 3 & 8 & 13 & \\
\hline LF & 0 & 3000 & $>3000$ & 1 & 5 & 3 & \\
\hline HF & 0 & 3000 & $>3000$ & 2 & 1 & 2 & \\
\hline Total & 0 & 6000 & $>6000$ & 2 & 12 & 2 & \\
\hline Ln LF/HF & & & & & & 1 & \\
\hline BMI & 0 & 100 & $>100$ & & & & 1 \\
\hline LVEF & 0 & 100 & $>100$ & & & & 1 \\
\hline
\end{tabular}

Tabelle 3 Tabelle der Definition für Ausreißer vor Logarithmierung einzelner HRV-Parameter. Zusätzlich Anzahl der anhand dieser Parameter identifiezierten Ausreißer pro Phase der EKG-Untersuchung.

\subsubsection{Ausschlüsse von EKG-Phasen}

In den drei EKG-Untersuchungsphasen mussten aufgrund der in den vorangegangenen Unterkapiteln aufgeführten Definitionen einige EKGs phasenweise ausgeschlossen werden. Es verblieben 421-444 Fälle pro Untersuchungsphase zur Analyse. 


\subsubsection{Probandenkollektiv}

Unter Beachtung der somatischen Ausschlusskriterien dieser Teilstudie (siehe unter 2.1.2.2 Ausschlusskriterien, S.19) waren von den 1286 Probanden des Kollektivs des KNHI in Göttingen für diese Teilstudie 1043 mögliche Probanden. Von diesen wurden 541 für die vorliegende Studie erreicht und stimmten der Teilnahme zu. Diese Gruppe wird als „LZ-EKGKollektiv“ bezeichnet.

Von diesen 541 Probanden wurde anhand der LZ-EKG-Daten eine erneute Kontrolle auf Rhythmusauffälligkeiten durchgeführt (s. 2.5.2.1 Rhythmusausschlüsse, S.30). Zusätzlich erfolgte eine Prüfung auf Ausreißer und ein Ausschluss bei zu vielen fehlenden LZ-EKGDaten (s. 2.5.2.2 Ausreißer, S.31). Es wurden aufgrund der beschriebenen Kriterien 7 Probanden ausgeschlossen. Die verbleibenden 534 Probanden werden als „LZ-EKG-Kollektiv rhythmusbereinigt" bezeichnet.

Aufgrund organisatorischer Ausschlusskriterien mussten 11 Probanden ausgeschlossen werden: zehn aufgrund zu alter Fragebögen, einer wegen zu kurzer Gehtestdauer.

In 21 Fällen war kein LZ-EKG mehr zur Auswertung zu finden. Davon lag bei 12 Datensätzen ein technischer Fehler beim Erheben vor. In fünf Fällen war aus zufälligen Gründen kein LZ-EKG durchgeführt worden, obwohl Probanden zum LZ-EKG-Raum gelangt waren. In vier Fällen ist unbekannt, warum keine LZ-EKG-Daten vorhanden waren. Vermutlich handelt es sich um Speicherschäden der verwendeten CD-Speichermedien.

Bei sechs Fällen standen keine vollständigen kardiologischen Daten zur Verfügung beziehungsweise war eine eindeutige Zuordnung zwischen den LZ-EKG-Probandendaten und den kardiologischen Daten nicht möglich.

In 28 Fällen lagen keine Fragebogendaten zur Analyse vor.

Es verblieben 468 Fälle zur weiteren Betrachtung: ,,analysiertes Kollektiv“ (s. Abb. 1 auf der folgenden Seite). 
Abbildung 1 Flow sheet des schrittweisen Ausschlusses von Probanden bis zum tatsächlich analysierten Probandenkollektiv

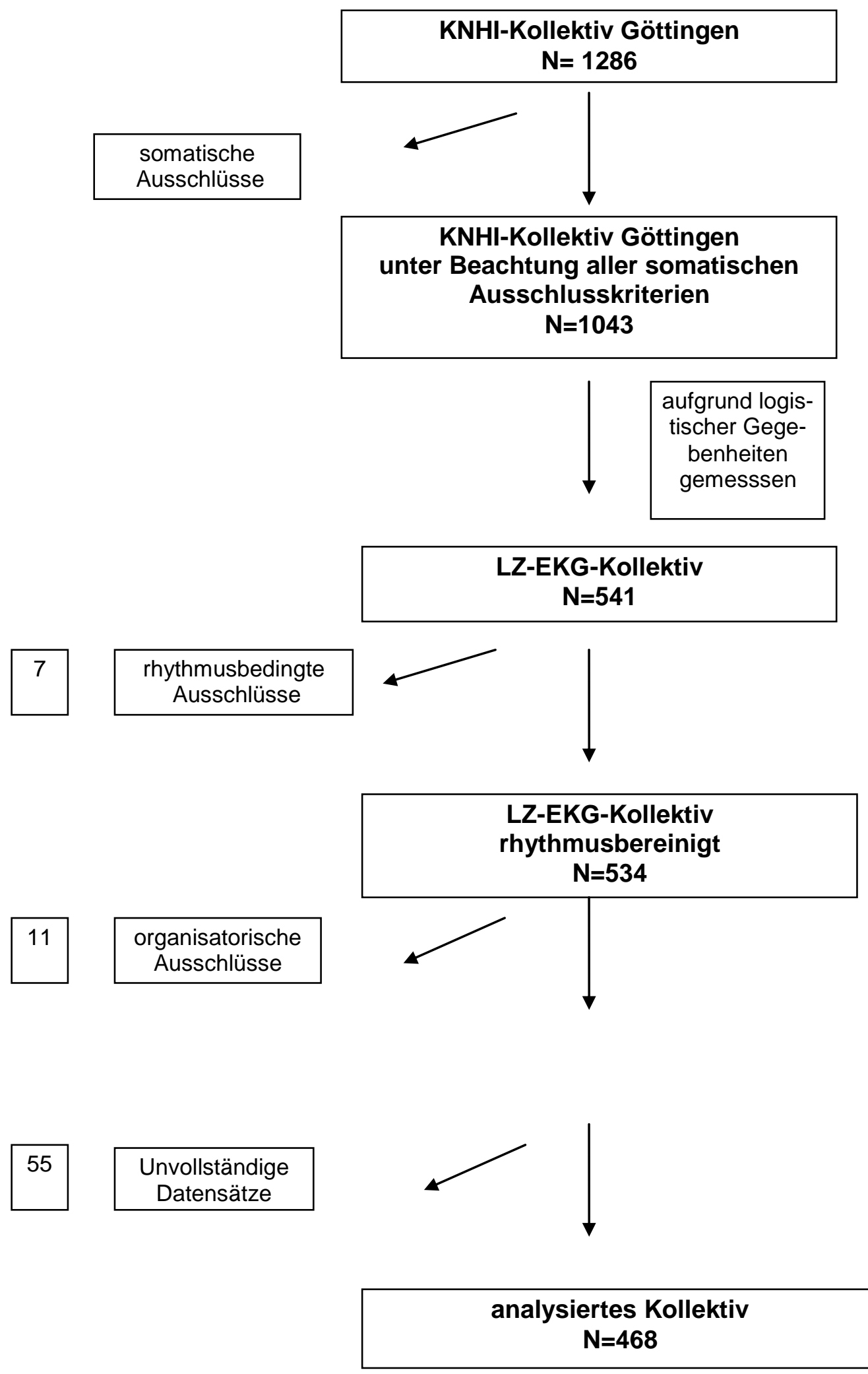




\subsubsection{Repräsentativitätsprüfung}

Zur Prüfung auf Repräsentativität des untersuchten $(\mathrm{N}=541)$, des rhythmusbereinigten $(\mathrm{N}=534)$ beziehungsweise des analysierten Kollektivs $(\mathrm{N}=468)$ bezogen auf die Gesamtheit des für diese Teilstudie nach Ausschlusskriterien zugelassenen Kollektivs (N=1043) wurde eine T-Testung mit getrennten Varianzen für die stetigen Parameter und eine Chi-QuadratTestung für die dichotomene Parameter durchgeführt.

Als Vergleichsparameter wurden die stetigen Parameter Alter, Werte des HADS für Angst und Depression, Werte des MQ, Werte der körperlichen Funktionsfähigkeit im SF-36 und die dichotomen Parameter Geschlecht, Vorerkrankungen HI, Koronare Herzkrankheit (KHK),DM und Zustand nach Herzinfarkt (MI) verwendet. Die Häufigkeit der Herzinsuffizienz zeigte in der Chi-Quadrat-Testung eine Abhängigkeit vom Kollektiv. Jeweils für die in die LZ-EKGUntersuchung einbezogene Gruppe zeigten sich weniger Fälle von Herzinsuffizienz. Ansonsten stellt das mittels LZ-EKG untersuchte Teilkollektiv ein repräsentatives Unterkollektiv dar. Die geringere Häufigkeit der Herzinsuffizienz könnte an der geringeren Motivierbarkeit der Probanden liegen.

\subsection{Statistische Datenauswertung}

Die Auswertung erfolgte durch Vera C. Duden mit Beratung durch den psychosomatischen Statistikbetreuer sowie die statistische Beratungsstelle der Universität Göttingen mittels Statistika 9 für Windows.

\subsubsection{Auswertungsplan}

Im Rahmen der deskriptiven Statistik wird das untersuchte Kollektiv, das unter Beachtung aller Ausschlusskriterien betrachtet werden konnte, beschrieben. Besonders beachtet werden dabei Geschlecht, Alter, Vorerkrankungen, psychische Erkrankungen, echokardiographische Parameter, NT-pro-BNP, Gehtestparameter und aktuelle Medikation. Die diesbezüglichen Beobachtungen werden mit Häufigkeitstabellen, Median, Mittelwert und Standardabweichung dargestellt. Nach Überlegungen zu Normalverteilung und Unabhängigkeit der im Rahmen der Hypothesenprüfung verwendeten Parameter erfolgt die Bearbeitung der Hypothesen. Die Auswertung der Hypothesen erfolgt mit den im Weiteren aufgeführten statistischen Verfahren. Beobachtungseinheiten mit fehlenden Daten wurden bei den Analysen von Zusammenhängen ausgeschlossen, so dass den Gruppenvergleichen jeweils variierende Fallzahlen zu- 
grunde liegen können. Bei den einzelnen Fragebögen ergeben sich unterschiedlich große Kollektive, da nicht alle Probanden den KNHI-Fragebogen vollständig ausfüllten. Bei den psychologischen Werten wird sowohl die stetige Skala als auch die dichotomisierte Bewertung der Fragebögen in der Analyse verwendet.

\subsubsection{Normalverteilung}

Die vorliegende Arbeit betrachtet als Endpunkte im Wesentlichen stetige Parameter. Für diese ist ab einer Gruppengröße über 30 eine Normalverteilung als Voraussetzung für die statistische Testung nicht mehr zwingend erforderlich. Für die dichotomen und kategorialen Zielparameter wurden nicht-parametrische Varianten der Verfahren verwendet. Teilweise wurde eine Logarithmierung zur Basis e (Logarithmus naturalis= Ln) zur Annäherung an eine Normalverteilung durchgeführt.

\subsubsection{Unabhängigkeit}

Bei den erhobenen Daten besteht innerhalb der HR und HRV-Parameter eine serielle Abhängigkeit, da an derselben Versuchsperson unter drei Versuchsbedingungen gemessen wurde. Zwischen den untersuchten Probanden wird von einer Unabhängigkeit der Daten ausgegangen.

\subsubsection{Statistische Tests}

Im Rahmen der statistischen Testung wurden relevante Werte auf zwei Stellen hinter dem Komma mathematisch gerundet. Das Signifikanzniveau wurde durch Sterne markiert. Signifikanzen auf dem $5 \%$-Niveau mit einem Stern (,signifikant“), auf dem 1\%-Niveau mit zwei Sternen (,hoch signifikant“) und auf dem 0,1\%-Niveau mit drei Sternen (,höchst signifikant") gekennzeichnet.

\section{Allgemeine Lineare Modelle}

Hierzu zählen Varianzanalysen (,analysis of varianz“=ANOVA) und Regressionsanalysen. Bei Statistika 9 werden unter dem Menüpunkt „Allgemeine Lineare Modelle“ auch kombinierte Verfahren angeboten.

\section{Varianzanalyse}

Bei der Untersuchung von Mittelwertsunterschiede zwischen Gruppen im Rahmen der Hypothesenprüfung wurden Varianzanalysen bei Statistika unter „Allgemeine Lineare Modelle“ gerechnet. Um die Messwertverteilung der einzelnen Messphasen auf Unterschiedlichkeit zu 
testen, wurden Mehrfach-Paarvergleiche in Form von Messwiederholungs-ANOVAs durchgeführt. Bei signifikanten Haupt-oder Interaktionseffekten wurde das Kollektiv nach Untersuchungszeitpunkt aufgeschlüsselt und erneut auf einen Mittelwertsunterschied analysiert.

\section{Regressionsanalysen}

Bei der Untersuchung des Zusammenhangs zwischen zwei stetigen Variablen im Rahmen der Hypothesenprüfung wurde eine lineare Regression gerechnet. Die Untersuchung wird genutzt, um eine Variable anhand von einem oder mehreren Prädiktoren vorherzusagen. Nach theoretischer Festlegung ist immer nur eine Richtung möglich: von unabhängigen Variablen zu abhängigen Variablen. Für lineare Regressionsmodelle wird ein linearer Zusammenhang zwischen den Variablen angenommen. Der Regressionskoeffizient (Beta) beschreibt die Richtung des linearen Zusammenhangs als Steigung der Geraden. Die Regressionskoeffizienten werden mittels der Methode der kleinsten Quadrate geschätzt. Wird nur ein Prädiktor benutzt, spricht man von einfacher, bei mehrere Prädiktoren von multipler Regression. Bei mehreren Einflussfaktoren wird die jeweils unabhängige Wirkung der Prädiktoren auf die abhängige Variable untersucht.

\section{Korrelationsmatrix}

Eine Korrelation beschreibt die Stärke und Richtung des linearen Zusammenhangs zwischen zwei Variablen. Für nicht stetige Parameter wurde zur Beschreibung des (nicht zwingend linearen) Zusammenhangs zwischen zwei Variablen ein Rangkorrelationskoeffizient nach Kendalls Tau verwendet. Minimale Voraussetzung hierfür ist eine Ordinalskalierung.

\section{T-Test}

Ein t-Test kann zum Mittelwertvergleich zwischen zwei Gruppen verwendet werden. Untersucht wird, ob die vorliegenden Mittelwertunterschiede, anhand eines vorher definierten Signifikanzniveaus - in dieser Arbeit 5\% - auf rein zufälligen Unterschieden basieren. In dieser Arbeit wurde mit getrennten Varianzschätzungen gerechnet.

\section{Pearsons Chi-Quadrat-Test}

Mit diesem Verfahren kann die Unabhängigkeit von diskreten Variablen getestet werden. Hierzu wird eine Kreuztabelle verwendet. Die Nullhypothese lautet, dass die Zeilen- und Spaltenvariablen voneinander unabhängig sind. Liegt der p-Wert unterhalb des definierten Signifikanzniveaus (hier 5\%) so muss die Nullhypothese abgelehnt werden. Damit ist von einer Abhängigkeit der Variablen auszugehen.

Um eine übersichtliche Darstellung der Daten zu gewährleisten, wurde eine tabellengestützte Präsentation angestrebt. 


\section{Ergebnisse}

\subsection{Deskriptive Statistik}

Das untersuchte Kollektiv ist ein Teilkollektiv des Göttinger KNHI Kollektivs. Die Beschreibung des Einschlussverfahrens sowie die Repräsentativitätsprüfung befinden sich im Teil 2 zu Probanden und Methoden (ab S.18).

\subsubsection{Somatische Kollektivbeschreibung}

Das analysierte Kollektiv besteht aus insgesamt 468 Probanden. Davon sind 220 Männer im Alter zwischen 51 und 84 Jahren (im Mittel 66,3 +/-7,6 Jahre) und 248 Frauen im Alter von 50 und 87 Jahren (im Mittel 67,3 +/-7,9 Jahre).

\subsubsection{Kardiale Vorerkrankungen, Risikofaktoren, Medikamente}

Im Rahmen der kardiologischen Untersuchung wurden Vorerkrankungen, kardiovaskuläre Risikofaktoren und Vormedikation erfragt beziehungsweise erhoben. Die für diese Teilstudie zur Einschätzung der kardiologischen Situation relevanten sind in Tabellen 4 und 5 nach Geschlecht getrennt aufgeführt.

\begin{tabular}{ccccccc}
\hline & Gesamt & $\begin{array}{c}\text { \% von } \\
\text { N }\end{array}$ & Männer & \% von & Frauen & \% von \\
\hline HTN & 408 & 87,18 & 189 & 85,91 & 219 & $\mathbf{N}$ \\
\hline DM & 111 & 23,72 & 64 & 29,09 & 47 & 18,95 \\
\hline $\begin{array}{c}\text { Cerebro- } \\
\text { vaskuläre } \\
\text { Erkran- } \\
\text { kung }\end{array}$ & 36 & 7,69 & 23 & 10,45 & 13 & 5,24 \\
\hline KHK & 83 & 17,74 & 49 & 22,27 & 34 & 13,71 \\
\hline pAVK & 23 & 4,91 & 17 & 7,73 & 6 & 2,42 \\
\hline Z. n. MI & 33 & 7,05 & 26 & 11,82 & 7 & 2,82 \\
\hline HI & 55 & 11,80 & 21 & 9,55 & 34 & 13,71 \\
\hline
\end{tabular}

Tabelle 4 Häufigkeit der Erkrankung „Herzinsuffizienz“ und kardialer Risikoerkrankungen für Herzinsuffizienz. Bei den Männern zwei Missing für den Parameter „Herzinsuffizienz“; sonst keine fehlenden Werte. N Gesamt 468, Männer 220, Frauen 248. 


\begin{tabular}{ccccc}
\hline \multicolumn{2}{c}{$\begin{array}{c}\text { BMI in kg/m } \\
\text { Mittelwert +/-SD }\end{array}$} & $28,97+/-4,71$ & $29,15+/-4,13$ & $28,79+/-5,16$ \\
\hline $\begin{array}{l}\text { Rauchen } \\
\text { Anzahl } \\
(\% \text { von N) }\end{array}$ & Ja & $36(7,71)$ & $19(8,68)$ & $17(6,85)$ \\
\cline { 2 - 5 } & Nein & $191(40,90)$ & $136(62,10)$ & $55(22,18)$ \\
\hline $\begin{array}{c}\text { Alkoholmenge in dpw } \\
\text { Mittelwert +/-SD }\end{array}$ & $5,09+/-8,52$ & $7,90+/-10,73$ & $2,60+/-4,69$ \\
\hline $\begin{array}{c}\text { Einnahme Betablocker } \\
\text { Anzahl (\% von N) }\end{array}$ & $232(49,68)$ & $107(48,64)$ & $125(50,61)$ \\
\hline $\begin{array}{c}\text { Einnahme Antiarrhythmika } \\
\text { Anzahl (\% von N) }\end{array}$ & $9(1,92)$ & $4(1,82)$ & $5(2,02)$ \\
\hline
\end{tabular}

Tabelle 5 Mittelwerte und Häufigkeiten von Risikofaktoren und Vormedikation. Für beide Geschlechter jeweils 2 fehlende Werte für den Parameter „BMI“, für Männer ein fehlender Wert für „Rauchstatus“, 3 fehlende Werte im Gesamtkollektiv bezüglich „Alkoholmenge in dpw“, davon 1 männlicher und 2 weibliche Probanden. Für jeweils einen weiblichen Probanden lagen bezüglich der Vormedikation mit Betablocker beziehungsweise Antiarrhythmika keine Informationen vor. N Gesamt 468, Männer 220, Frauen 248.

\subsubsection{Kardiale Schweregradmarker}

Im Folgenden sind die Mittelwerte und Häufigkeiten der Parameter aus der Echokardiographie (LVEF, diastolischer Herzinsuffizienzscore), das NT-pro-BNP sowie das Ergebnis des 6Minuten-Gehtests aufgeführt (s. Tabelle 6 auf der folgenden Seite). Das NT-pro-BNP wurde zur Annäherung an eine Normalverteilung für die analytische Statistik zur Basis e (Ln) logarithmiert. 


\begin{tabular}{ccccc}
\hline \multicolumn{2}{c}{$\begin{array}{c}\text { LVEF (in Prozent) } \\
\text { Mittelwert +/-SD }\end{array}$} & $59,22+/-7,42$ & $58,26+/-8,34$ & $60,08+/-6,39$ \\
\hline $\begin{array}{c}\text { Diastolischer } \\
\text { Herzinsuffizi- } \\
\text { enz- score }\end{array}$ & $\mathbf{0}$ & $111(23,72)$ & $51(23,18)$ & $60(24,19)$ \\
\cline { 2 - 5 } Anzahl (\%) & $\mathbf{1}$ & $251(53,63)$ & $114(51,82)$ & $137(55,24)$ \\
\hline NT-pro-BNP in pg/ml & $\mathbf{2}$ & $2(0,43)$ & $1(0,45)$ & $1(0,40)$ \\
Median ( unteres /oberes & $(51,64 / 181,10)$ & $(43,22 / 172,20)$ & $(64,56 / 185,80)$ \\
Quartil) & 97,34 & & 119,10 \\
\hline Gehteststreckenlänge (in m) & $542,91+/-91,85$ & $567,18+/-$ & $521,38+/-81,76$ \\
Mittelwert +/-SD
\end{tabular}

Tabelle 6 Mittelwerte und Häufigkeiten von kardialen Schweregradmarkern. Bezüglich „LVEF“ fehlt der Wert für jeweils einen weiblichen und einen männlichen Probanden. Für, ,diastolischer Herzinsuffizienzscore“ fehlen 6 Werte für männliche Probanden, 1 Wert bei den weiblichen Probanden. „Diastolischer Herzinsuffizienzscore": 0 ist der beste Wert, 3 der schlechteste (s. 6.1 Diastolischer Herzinsuffizienzscore im KNHI, S.95). N Gesamt 468, Männer 220, Frauen 248.

Zusammenfassend besteht das Kollektiv aus etwas mehr Frauen als Männern. Die Frauen sind im Durchschnitt etwas älter. Bei Männern sind die kardiovaskulären Vorerkrankungen häufiger vertreten, der Alkoholkonsum ist höher, die Gehstrecke länger, die LVEF im Mittel niedriger. NT-pro-BNP zeigte bei den Frauen höhere Werte. Frauen nahmen etwas häufiger Betablocker sowie andere antiarrhythmisch wirkende Medikamente ein. Bei den Frauen sind mehr Fälle von HTN und HI vertreten. 


\title{
3.1.2 Psychische Kollektivbeschreibung
}

Im Folgenden werden die deskriptiven Daten der vier untersuchten psychischen Dimensionen aufgeführt. Beurteilt wurden die psychischen Dimensionen „Depressivität“, „Angst“, „Vitale Erschöpfung“ und „körperliche Funktionsfähigkeit“ (Teilaspekt der subjektiven Lebensqualität). Signifikante Geschlechtsunterschiede im t-Test mit getrennten Varianzen werden mit Sternen anhand des Signifikanzniveaus markiert und Abhängigkeit im Chi-Quadrat-Test durch kursive Schreibweise hervorgehoben.

Gesamt Männer $\quad$ Frauen

\section{Depressivität}

Mittelwert +/-SD

vorhanden:

$$
\begin{array}{ccc}
4,46+/-3,50 & 4,30+/-3,57 & 4,60+/-3,44 \\
62(13,25) & 30(13,64) & 32(12,90)
\end{array}
$$

Anzahl (\% von N)

\section{Angst}

Mittelwert +/-SD**

vorhanden:

$5,87+/-4,21$

$5,31+/-4,23$

$6,37+/-4,14$

$79(16,88)$

$31(14,09)$

$48(19,35)$

Anzahl (\% von $\mathrm{N}$ )

Vitale Erschöpfung

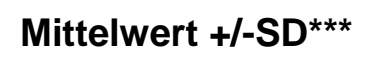

vorhanden:

$10,96+/-9,81$

$8,95+/-8,80$

$12,74+/-10,31$

$92(19,66)$

$34(15,45)$

$58(23,39)$

Anzahl (\% von N)

Körperliche Funktionsfähigkeit

\author{
Mittelwert +/-SD* \\ niedrig: \\ Anzahl (\% von N)
}

$76,58+/-21,83$

$84(17,95)$

$79,37+/-21,34$

$74,12+/-22,01$

$31(14,09)$

$53(21,37)$

Tabelle 7 Mittelwerte der stetigen Werte sowie Anzahl der Probanden mit als auffällig gewerteten Scores, beschrieben als Merkmal ,vorhanden“ beziehungsweise „niedrig“ anhand der im Methodikteil definierten cut-off-Werte (ab S.23). Mit t-Test wurde auf signifikante Unterschiede beziehungsweise mit ChiQuadrat-Test auf Abhängigkeit untersucht. Für „Depressivität“" fehlten jeweils 4 Werte für Männer und Frauen. Für „Angst“ fehlten 3 Werte bei den Männern, 4 bei den Frauen. Für „VE“ fehlten 22 Werte bei den Männern, 24 bei den Frauen. Für ,körperliche Funktionsfähigkeit“ fehlten 6 Werte der Männer, 5 der Frauen. N Gesamt 468, Männer 220, Frauen 248. Signifikante Mittelwertsunterschiede für die verschiedenen Geschlechter sind mit Sternen markiert $(\mathbf{p}<0,05=*, \mathbf{p}<0,01=* *, \mathbf{p}<0,001=* * *)$. Abhangigkeit im Chi-Quadrat-Test wird durch kursive Schreibweise hervorgehoben.

Insgesamt sind die Frauen signifikant ängstlicher, vital erschöpfter und geben eine schlechtere körperliche Funktionsfähigkeit an. 


\subsubsection{Herzfrequenz und Herzfrequenzvariabilität}

Im Folgenden sind die untersuchte HR und die HRV-Parameter mit Mittelwert und Standardabweichung aufgeführt.

Die mittlere Herzfrequenz, pNN50 sowie Ln LF/HF wurden so verwendet, wie von Medilog Darwin berechnet. Die restlichen Parameter wurden zur Annäherung an die Normalverteilung zur Basis e logarithmiert (Logarithmus naturalis =Ln) und daher auch so aufgeführt. Signifikante Unterschiede aus der t-Testung mit getrennten Varianzen im Geschlechtervergleich wurden mit Sternen anhand des Signifikanzniveaus markiert (s. 6.4 HRV nach Alter und Geschlecht, ab S.112).

\begin{tabular}{llllllllll}
\hline & \multicolumn{1}{l}{ Alle } & \multicolumn{3}{c}{ Männer } & \multicolumn{3}{l}{ Frauen } \\
\cline { 2 - 10 } & $\mathbf{N}$ & Mittelwert & SD & $\mathbf{N}$ & Mittelwert & SD & $\mathbf{N}$ & Mittelwert & SD \\
\hline $\begin{array}{l}\text { Gehphase ( 2 Minuten) } \\
\text { mittlere }\end{array}$ & 423 & 104,51 & 17,53 & 205 & 103,88 & 17,03 & 218 & 105,10 & 18,02 \\
\hline $\begin{array}{l}\text { HR } \\
\text { SDNN }\end{array}$ & 423 & 2,82 & 0,55 & 205 & 2,78 & 0,56 & 218 & 2,86 & 0,55 \\
\hline $\begin{array}{l}\text { Ln } \\
\text { rMSSD* }\end{array}$ & 423 & 2,78 & 0,83 & 205 & 2,67 & 0,88 & 218 & 2,87 & 0,76 \\
\hline pNN50 & 421 & 0,03 & 0,06 & 204 & 0,03 & 0,057 & 217 & 0,04 & 0,06 \\
\hline Ln LF & 423 & 2,31 & 1,36 & 205 & 2,28 & 1,37 & 218 & 2,33 & 1,36 \\
\hline Ln HF & 423 & 2,23 & 1,62 & 205 & 2,08 & 1,66 & 218 & 2,37 & 1,56 \\
\hline $\begin{array}{l}\text { Ln To- } \\
\text { tal }\end{array}$ & 423 & 3,91 & 1,20 & 205 & 3,89 & 1,21 & 218 & 3,94 & 1,18 \\
\hline $\begin{array}{l}\text { Ln } \\
\text { LF/HF* }\end{array}$ & 423 & 0,03 & 0,48 & 205 & 0,09 & 0,49 & 218 & $-0,01$ & 0,46 \\
\hline
\end{tabular}

Taktatmungsphase ( 5 Minuten)

\begin{tabular}{llllllllll}
\hline $\begin{array}{l}\text { mittlere } \\
\text { HR }\end{array}$ & 444 & 73,56 & 12,69 & 213 & 73,17 & 12,33 & 231 & 73,93 & 13,04 \\
\hline $\begin{array}{l}\text { Ln } \\
\text { SDNN }\end{array}$ & 442 & 3,53 & 0,52 & 212 & 3,49 & 0,53 & 230 & 3,58 & 0,50 \\
\hline $\begin{array}{l}\text { Ln } \\
\text { rMSSD }\end{array}$ & 444 & 3,10 & 0,79 & 213 & 3,03 & 0,78 & 231 & 3,16 & 0,79 \\
\hline pNN50 & 441 & 0,05 & 0,076 & 212 & 0,05 & 0,07 & 229 & 0,06 & 0,08 \\
\hline Ln LF & 443 & 5,20 & 1,13 & 213 & 5,14 & 1,18 & 230 & 5,26 & 1,08 \\
\hline Ln HF* & 444 & 3,87 & 1,39 & 213 & 3,73 & 1,37 & 231 & 3,99 & 1,40 \\
\hline $\begin{array}{l}\text { Ln To- } \\
\text { tal* }\end{array}$ & 437 & 6,65 & 0,99 & 208 & 6,54 & 1,06 & 229 & 6,75 & 0,92 \\
\hline $\begin{array}{l}\text { Ln } \\
\text { LF/HF }\end{array}$ & 443 & 0,58 & 0,46 & 213 & 0,61 & 0,46 & 230 & 0,56 & 0,46 \\
\hline
\end{tabular}


Ruhephase ( 5 Minuten)

\begin{tabular}{lccccccccc}
\hline $\begin{array}{l}\text { mittlere } \\
\text { HR }\end{array}$ & 431 & 72,49 & 12,29 & 205 & 72,04 & 12,24 & 226 & 72,89 & 12,35 \\
\hline Ln SDNN & 429 & 3,39 & 0,47 & 205 & 3,39 & 0,46 & 224 & 3,38 & 0,47 \\
\hline $\begin{array}{l}\text { Ln } \\
\text { rMSSD }\end{array}$ & 431 & 2,88 & 0,80 & 205 & 2,90 & 0,78 & 226 & 2,86 & 0,83 \\
\hline pNN50 & 431 & 0,03 & 0,05 & 205 & 0,03 & 0,04 & 226 & 0,03 & 0,06 \\
\hline Ln LF & 431 & 4,85 & 1,22 & 205 & 4,78 & 1,18 & 226 & 4,92 & 1,26 \\
\hline Ln HF & 431 & 3,39 & 1,34 & 205 & 3,24 & 1,25 & 226 & 3,52 & 1,40 \\
\hline Ln Total & 430 & 6,01 & 0,99 & 205 & 5,94 & 0,94 & 225 & 6,08 & 1,02 \\
\hline Ln LF/HF & 430 & 0,64 & 0,44 & 204 & 0,67 & 0,46 & 226 & 0,61 & 0,41 \\
\hline
\end{tabular}

Tabelle 8 Auflistung der Mittelwerte HR und HRV für das Gesamtkollektiv und nach Geschlecht getrennt. Die Parameter mit signifikanten Unterschieden im t-Test mit getrennten Varianzen zwischen Männern und Frauen wurden in der ersten Spalte mit Sternen für das jeweilige Signifikanzniveau markiert: $\mathbf{p}<0,05=*, \mathbf{p}<0,01=* *, \mathbf{p}<0,001=* * *$.

Sowohl bezüglich Alter als auch Geschlecht zeigten sich signifikante Unterschiede. Dies wird im Rahmen der Testung auf somatische Einflussparameter berücksichtigt.

Die Prüfung auf Mittelwertunterschiede der HR und der HRV-Parameter nach psychischer Auffälligkeit, gemessen anhand der aktuell erhobenen Fragebogenscores, erfolgte im Rahmen der Hypothesenprüfung (eine vollständige Auflistung der HR und HRV-Parameter nach psychischer Auffälligkeit findet sich im Anhang unter 6.5 HRV nach psychologischen Variablen ab S.121). 


\subsection{Hypothesenprüfung}

Im Rahmen der Hypothesenprüfung werden nur signifikante Ergebnisse beschrieben und weiter differenziert.

\subsubsection{Zusammenhang zwischen Depressivität und HR sowie HRV- Parametern (Hypothese 1)}

„Depressive Symptome A) gehen mit einem erhöhten Sympathikotonus und einem reduzierten Vagotonus einher, erkennbar an einer erhöhten HR und erniedrigten globalen und vagalen sowie erhöhten sympathischen HRV-Parametern. B) Dieser Zusammenhang ist unabhängig vom kardialen Status. C) Besonders ausgeprägt tritt er während der Taktatmungsphase auf. D) Diese Effekte werden für Männer und für Frauen erwartet.“

\subsubsection{Unterhypothese A}

Zur Prüfung der Hypothese 1A wird bei Statistika ein Allgemeines Lineares Modell mit Messwiederholungen gerechnet. Als abhängige Parameter wurden die HR und die HRVParameter in den drei Untersuchungsphasen (,ZEIT“) definiert. Unabhängiger Parameter ist das Vorhandensein oder Fehlen von depressiven Symptomen anhand der HADS. Die Ergebnisse dieser Analyse finden sich in Tabelle 9 auf der folgenden Seite. 


\begin{tabular}{cccc}
\hline & $\begin{array}{l}\text { Haupteffekt } \\
\text { Depressivität }\end{array}$ & $\begin{array}{l}\text { Haupteffekt } \\
\text { Zeit }\end{array}$ & $\begin{array}{l}\text { Interaktion } \\
\text { Zeit mit Depressivität }\end{array}$ \\
\hline mittlere HR & $<0,05^{*}$ & $<0,001^{* * *}$ & $<0,05^{*}$ \\
\hline Ln SDNN & 0,41 & $<0,001^{* * *}$ & 0,16 \\
\hline Ln rMSSD & 0,58 & $<0,001^{* * *}$ & 0,10 \\
\hline pNN50 & 0,79 & $<0,001^{* * *}$ & 0,90 \\
\hline Ln LF & 0,80 & $<0,001^{* * *}$ & $<0,01^{* *}$ \\
\hline Ln HF & 0,08 & $<0,001^{* * *}$ & 0,46 \\
\hline Ln Total & 0,28 & $<0,001^{* * *}$ & $<0,05^{*}$ \\
\hline Ln LF/HF & $<0,05^{*}$ & $<0,001^{* * *}$ & 0,07 \\
\hline
\end{tabular}

Tabelle 9 Allgemeines Lineares Modell mit Messwiederholungen von HR und HRV. Untersucht wurde „Depressivität“ als unabhängiger Parameter. Angegeben ist der p-Wert mit jeweiligem Signifikanzniveau $(\mathbf{p}<0,05=* ; \mathbf{p}<0,01=* * ; \mathbf{p}<0,001=* * *)$. Gruppengröße anhand dichotomisierter HADS schwankte aufgrund phasenweise fehlender Daten: „Depressivität auffällig“ N 56-58, „Depressivität nicht auffällig“ N 358-378.

Die HR und die HRV-Parameter mit signifikanten Haupt-oder Interaktionseffekten wurden im Anschluss nach Untersuchungsphasen getrennt erneut analysiert, um zu bestimmen, in welcher Phase tatsächlich ein signifikanter Mittelwertunterschied dem Haupteffekt zu Grunde liegt und ob hinter dem Interaktionseffekt ein signifikanter Mittelwertsunterschied in einer Phase verborgen ist.

Es zeigten sich in dieser Untersuchung für die in Tabelle 9 aufgezeigten signifikanten Hauptund Interaktionseffekte die in Tabelle 10 aufgeführten signifikanten Effekte.

\section{Gehphase Taktatmungsphase Ruhephase}

\begin{tabular}{cccc}
\hline mittlere HR & $<0,01^{* *}$ & --- & -- \\
\hline Ln LF/HF & --- & $<0,05^{*}$ & $<0,01^{* *}$
\end{tabular}

Tabelle 10 Einzelphasenanalyse für signifikante Effekte der Depressivität wie aufgeführt in Tabelle 9 für Gruppierungsvariable „Depressivität“. Nur signifikante Ergebnisse sind hier aufgeführt worden. Signifikanzniveau anhand von Sternen markiert ( $\mathbf{p}<0,05=* ; \mathbf{p}<0,01=* * ; \mathbf{p}<0,001=* * *)$.

Für die Parameter mit signifikanten Effekten in Tabelle 10 wurden Diagramme erstellt (s. Abb. 2 mittlere HR und Abb. 3 Ln LF/HF auf der folgenden Seite) und eine weitergehende Analyse im Rahmen der Unterhypothesen angeschlossen. 
Abbildung 2 Mittelwerte der mittleren HR mit der Gruppierungsvariablen „Depressivität“" in allen drei Untersuchungsphasen. Für die Gehphase bestand ein Mittelwertunterschied auf $1 \%$ Signifikanzniveau.

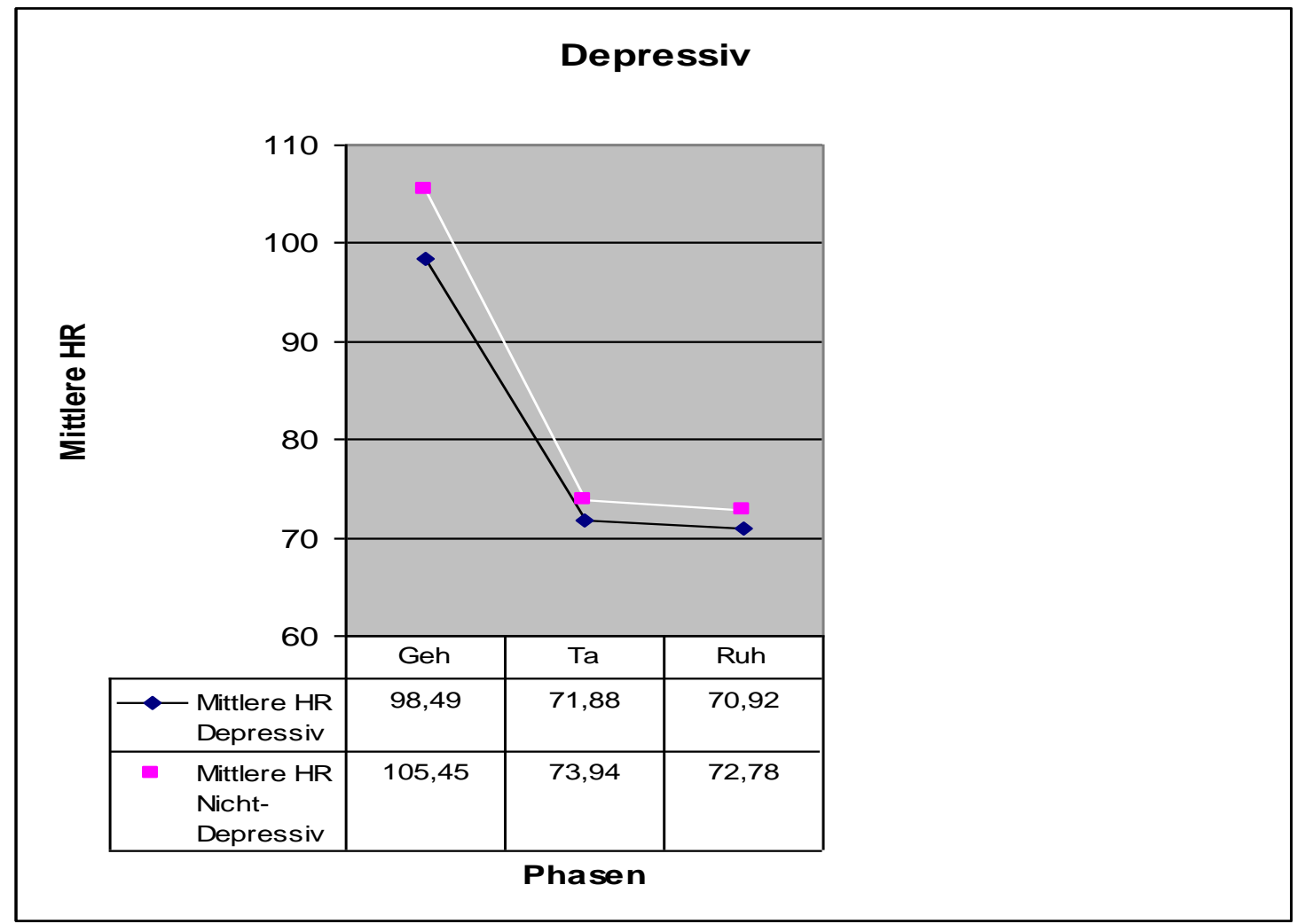

Abbildung 3 Mittelwerte der Ln LF/HF mit der Gruppierungsvariablen „Depressivität“ in allen drei Untersuchungsphasen. Für die Taktatmungsphase zeigte sich ein signifikanter Mittelwertunterschied auf 5\% Signifikanzniveau, für die Ruhephase auf $1 \%$ Signifikanzniveau

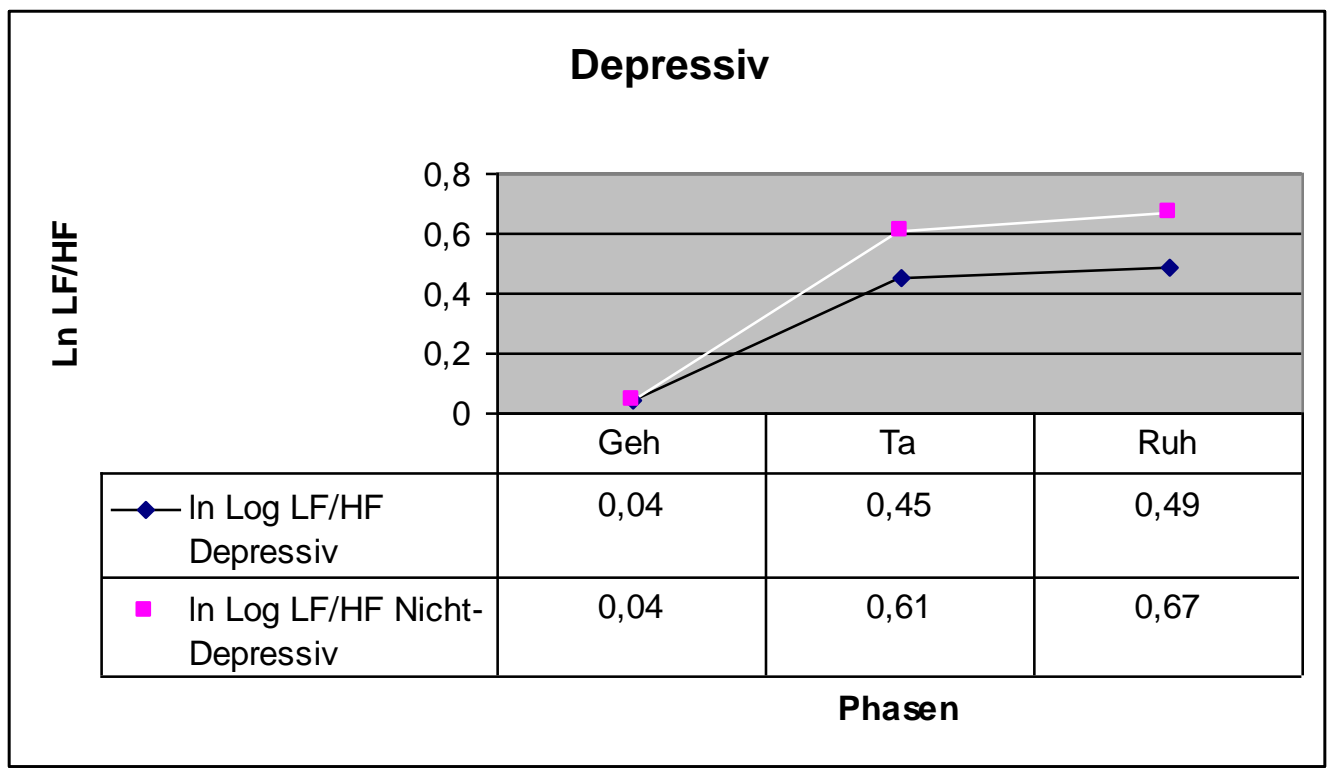


Für die mittlere Herzrate ließ sich in der Gehphase ein signifikanter Mittelwertsunterschied zwischen Depressiven und Nicht-Depressiven nachweisen (s. Tabelle 10 (S.44), s. Abb. 2 (S.45)). Die Werte der Depressiven waren niedriger als die der Nicht-Depressiven. Die in Hypothese 1A vermutete Dominanz des Sympathikus mit daraus resultierender höherer mittlerer Herzrate bei Depressiven ließ sich also nicht bestätigen.

Der niedrigere Durchschnittswert für mittlere Herzrate der Depressiven in der Gehphase könnte einem Motivationsmangel mit daraufhin geringerer körperlicher Anstrengung und damit auch geringerer Aktivierung des Sympathikus geschuldet sein.

Die Ln LF/HF zeigte in Taktatmungs- und Ruhephase signifikant erniedrigte Werte für die Depressiven. In der Taktatmungsphase könnten die niedrigen Werte für Ln LF/HF bei den Depressiven ein Zeichen erniedrigter Resonanzfähigkeit oder aber auch mangelnder Kooperation bei der Taktatmung sein. Durch die Taktatmung wäre ein Anstieg des LF und damit auch des LF/HF Quotienten erwartet worden. Ln LF/HF wird teilweise als Marker für die Modulationen des Sympathikus beschrieben (TASK FORCE OF THE EUROPEAN SOCIETY OF CARDIOLOGY AND THE NORTH AMERICAN SOCIETY OF PACING AND ELECTROPHYSIOLOGY 1996). Ein erhöhter Sympathikotonus, also hohe Werte für Ln LF/HF, wie in der Hypothese erwartet, lagen für die Depressiven nicht vor.

Diese Ergebnisse entsprechen nicht den erwarteten Ergebnissen einer autonomen Dysfunktion für die Depressiven. Unterhypothese A muss daher verworfen werden.

Explorativ wurde eine Analyse analog der Analyse in Hypothese 1A (S.43) mit den stetigen Werten der Depressivitätsskala durchgeführt. Hier zeigten sich keine signifikanten Haupteffekte.

\subsubsection{Unterhypothese B}

Um zu prüfen, ob der in Hypothese 1A (S.43) für den Gruppierungsparameter „Depressivität“ beobachtete signifikante Mittelwertsunterschied für mittlere HR in der Gehphase und Ln LF/HF in der Taktatmungs- und Ruhephase in einem Modell mit somatischen Einflussfaktoren erhalten bleibt, wird ein neues Modell mit diesen Parametern formuliert. 
Im Allgemeinen Linearen Modell werden zunächst die Werte von mittlerer HR und Ln LF/HF jeweils als abhängige Parameter in den Phasen angegeben, in denen sie in Hypothese 1A (S.43) einen signifikanten Unterschied zeigten.

Primär wird als kategorialer Prädiktor „Depressivität vorhanden“ beziehungsweise „nicht vorhanden“ angegeben. Im Anschluss wird jeweils ein somatischer Faktor als stetiger beziehungsweise kategorieller Prädiktor zusätzlich in das Modell integriert. Die analysierten somatischen Faktoren sind: Alter, Geschlecht, BMI, Vorerkrankung HI, KHK, DM, HTN, Z.n. MI, pAVK, cerebrovaskuläre Vorerkrankung, Rauchstatus, Alkoholmenge pro Woche, Vormedikation Betablocker beziehungsweise Antiarrhythmika, LVEF, diastolischer Herzinsuffizienzscore, Uhrzeit der Langzeit-EKG-Testung, Gehteststreckenlänge und Höhe des logarithmierten NT-pro-BNP.

Im Weiteren werden lediglich Confounder/Mediatoren aufgelistet (wenn ein Parameter einen signifikanten Effekt zeigt, die Depressivität dagegen keinen unabhängigen Effekt aufweist) beziehungsweise die Parameter, die neben dem psychischen Parameter einen unabhängigen Effekt haben (beide zeigen einen signifikanten Zusammenhang).

\subsection{Mittlere HR}

Als mögliche Mediatoren oder Confounder des Effekts der Depressivität im Rahmen der unter 3.2.1.2 Unterhypothese B (S.46) beschriebenen Testung im Allgemeinen Linearen Modell zeigten sich die Vorerkrankung HTN und die Länge der Gehteststrecke. Mit jeweils einem dieser Parameter im Modell zeigte sich für Depressivität kein signifikanter Einfluss mehr auf die mittlere HR in der Gehphase.

Einen jeweils unabhängigen Einfluss auf die mittlere HR in der Gehphase im Rahmen der Testung wie unter 3.2.1.2 Unterhypothese B (S.46) hatte zusätzlich zur Depressivität die Vormedikation mit Betablockern, das Alter, KHK, der diastolische Herzinsuffizienzscore und das Ln NT-pro-BNP. Für diese Parameter zeigte sich in der Gehphase jeweils ein unabhängiger signifikanter Einfluss für Depressivität und jeweils einen der oben genannten Parameter, die jeweils einzeln zu Depressivität in das Modell eingefügt worden waren.

\subsection{Ln LF/HF}

Als mögliche Mediatoren oder Confounder des Effekts der Depressivität in der Taktatmungsund Ruhephase zeigten sich in der Taktatmungsphase im Rahmen eines Allgemeinen Linearen Modells, wie unter 3.2.1.2 Unterhypothese B (S.46) beschrieben, gerechnet, die Vormedi- 
kation mit Antiarrhythmika und in der Ruhephase die Vorerkrankung Herzinsuffizienz. Neben diesen jeweils einzeln einfügten Parametern war der Einfluss der Depressivität auf die Ln LF/HF nicht mehr signifikant.

Einen jeweils unabhängigen Einfluss auf die Ln LF/HF in der Taktatmungsphase hatten zusätzlich zur Depressivität im Rahmen des Allgemeinen Linearen Modells, wie unter 3.2.1.2 Unterhypothese B (S.46) berechnet, die folgenden, jeweils einzeln in das Modell zu Depressivität eingefügten, Variablen: die Gehteststrecke in Metern, Vormedikation mit Betablocker, Ln NT-pro-BNP, das Alter, LVEF, Vorerkrankung MI und die Alkoholmenge pro Woche.

In der Ruhephase zeigten das Alter, Geschlecht, Alkoholmenge pro Woche, Vormedikation mit Betablocker, der diastolische Herzinsuffizienzscore, die Gehteststreckenlänge und das Ln Nt-pro-BNP einen unabhängigen Einfluss neben der Depressivität im Rahmen des unter 3.2.1.2 Unterhypothese B (S.46) beschriebenen Allgemeinen Linearen Modells auf die LF/HF.

Die Vorerkrankung HTN sowie die Gehteststreckenlänge zeigte einen Mediatorbeziehungsweise Confoundereffekt auf die mittlere HR in der Gehphase. Diese Phase war in der Prüfung der Unterhypothese 1 A als Phase mit signifikantem Mittelwertunterschied für Depressivität identifiziert worden (s. Tabelle 10, S.44).

Lediglich die Vormedikation mit Antiarrhythmika zeigte einen Mediator-beziehungsweise Confoundereffekt auf die Ln LF/HF in der Taktatmungsphase. Diese Phase war neben der Ruhephase als eine Phase mit einem signifikanten Unterschied im Rahmen der Prüfung von Hypothese 1 A beschrieben worden (s. Tabelle 10, S.44). In der Ruhephase zeigte lediglich die Vorerkrankung HI einen Mediator- beziehungsweise Confoundereffekt auf die Ln LF/HF. Viele der somatischen Faktoren und der kardialen Schweregradmarker wiesen eine unabhängige Signifikanz neben der Depressivität auf. Für diese Faktoren ist ein Teil des Depressivitätseffekts unabhängig von den somatischen Variablen.

Unterhypothese 1B muss für die Vorerkrankung HTN und die Gehteststreckenlänge bei mittlerer HR und für die Vormedikation mit Antiarrhythmika sowie die Vorerkrankung HI für Ln LF/HF verworfen werden. Von anderen kardialen Erkrankungen und kardialen Schwergradmarkern erscheint der Einfluss der Depressivität auf die mittlere HR und die Ln LF/F zumindest teilweise unabhängig zu sein. 


\subsubsection{Unterhypothese C}

Es war erwartet worden, dass die unter 3.2.1.1 Unterhypothese A aufgezeigten Effekte der Depressivität auf die HR und HRV besonders deutlich in der Taktatmungsphase zu beobachten sind. Dies war nicht der Fall (s. Tabelle 10, S.44). Unterhypothese $1 \mathrm{C}$ muss daher abgelehnt werden.

\subsubsection{Unterhypothese D}

Die in Hypothese 1A beschriebenen Effekte (s. Tab. 10, S.44) werden sowohl für Männer als auch für Frauen erwartet.

Es wurde ein Allgemeines Lineares Modell, wie unter 3.2.1.1 Unterhypothese A (S.43) beschrieben, für mittlere HR und Ln LF/HF durchgeführt, da diese in Unterhypothese 1A als Parameter mit einem signifikanten Haupteffekt der Depressivität identifiziert worden waren (s. Tab. 10, S.44). Jeweils wurde die Untersuchung mit Selektion für nur Männer beziehungsweise nur Frauen durchgeführt.

Für mittlere HR zeigte sich für die Gesamtkohorte der unter Unterhypothese 1A beschriebene Haupt- und Interaktionseffekt. In der Analyse für Männer beziehungsweise für Frauen zeigte sich weder ein Haupt- noch ein Interaktionseffekt.

Für die Ln LF/HF zeigt sich in der Gesamtkohorte der unter Unterhypothese 1A beschriebene Haupteffekt, kein Interaktionseffekt. In der Analyse für Männer zeigte sich weder ein Hauptnoch ein Interaktionseffekt. Für Frauen zeigten sich sowohl ein Haupt- als auch ein Interaktionseffekt.

Unterhypothese $1 \mathrm{D}$ muss abgelehnt werden, da bei geschlechtsgetrennter Auswertung die nicht hypothesenkonformen signifikanten Effekte aus Hypothese 1A (s. Tab. 10, S.44) für mittlere HR nicht, für Ln LF/HF nur für Frauen nachgewiesen werden konnten.

Zusammenfassend zeigte sich für Depressivität ein signifikanter Haupteffekt für mittlere HR und Ln LF/HF, allerdings keine Sympathikusdominanz oder Vagussuppression. Dieser signifikante Haupteffekt war nur teilweise unabhängig vom somatischen Status (1B), in der Taktatmungsphase nicht besonders ausgeprägt (1C) und ließ sich in der geschlechtergetrennten Betrachtung nur teilweise nachweisen (1D).

\section{Hypothese 1 muss daher abgelehnt werden.}




\subsubsection{Zusammenhang zwischen Angst, Vitaler Erschöpfung, körperlicher Funktionsfähigkeit und HR sowie HRV-Parametern (Hypothese 2)}

„Ebensolche Zusammenhänge wie für Depressivität mit HR und HRV werden für Angst, Vitale Erschöpfung und niedrige Lebensqualität angenommen. A) Erwartet wird ein erhöhter Sympathikotonus und ein reduzierten Vagotonus, erkennbar an einer erhöhten HR und erniedrigten globalen und vagalen sowie erhöhten sympathischen HRV-Parametern. B) Dieser Zusammenhang ist unabhängig vom kardialen Status. C) Besonders ausgeprägt tritt er während der Taktatmungsphase auf. D) Diese Effekte werden für Männer und für Frauen erwartet.“

\subsubsection{Angst}

\subsection{Unterhypothese A}

Untersucht wurde die Beziehung zwischen der HR und den HRV-Parametern (abh.) und „Angst vorhanden/nicht vorhanden“ (unabh.) in einem Allgemeinen Linearen Modell mit Messwiederholungen.

\begin{tabular}{cccc}
\hline & $\begin{array}{c}\text { Haupteffekt } \\
\text { Angst }\end{array}$ & $\begin{array}{c}\text { Haupteffekt } \\
\text { Zeit }\end{array}$ & $\begin{array}{c}\text { Interaktion } \\
\text { Zeit mit Angst }\end{array}$ \\
\hline mittlere HR & 0,77 & $<0,001^{* * *}$ & 0,88 \\
\hline Ln SDNN & 0,94 & $<0,001^{* * *}$ & 0,19 \\
\hline Ln rMSSD & 0,98 & $<0,001^{* * *}$ & 0,39 \\
\hline pNN50 & 0,23 & $<0,01^{* *}$ & 0,45 \\
\hline Ln LF & 0,98 & $<0,001^{* * *}$ & 0,28 \\
\hline Ln HF & 0,34 & $<0,001^{* * *}$ & 0,37 \\
\hline Ln Total & 0,77 & $<0,001^{* * *}$ & 0,09 \\
\hline Ln LF/HF & 0,16 & $<0,001^{* * *}$ & 0,97 \\
\hline
\end{tabular}

Tabelle 11 Allgemeines Lineares Modell mit Messwiederholungen von HR und HRV. Untersucht wurde die Gruppierungsvariable „Angst“6 als unabhängiger Parameter. Angegeben ist der jeweilige p-Wert mit Signifikanzniveau $(\mathbf{p}<0,05=* ; \mathbf{p}<0,01=* * ; p<0,001=* * *)$. Gruppengröße anhand dichotomisierter HADSAngstskala schwankte aufgrund fehlender Daten: Angst vorhanden N 70-77, Angst nicht vorhanden N 345-360.

Es zeigten sich kein Haupteffekt der Angst und kein signifikanter Interaktionseffekt. Daher wurde auf weitere Testungen verzichtet. Eine explorative Untersuchung mit den stetigen Rohwerten der HADS-Angstskala erbrachte ebenfalls keine Haupt-oder Interaktionseffekt.

Hypothese 2 für Angst muss verworfen werden. 


\subsubsection{Vitale Erschöpfung}

\subsection{Unterhypothese A}

Bezüglich des Parameters „Vitale Erschöpung“ zeigte sich in der primär durchgeführten Analyse mit dichotomisiertem Parameter im Rahmen eines Allgemeinen Linearen Modells mit Messwiederholungen kein Haupteffekt.

Daraufhin wurde eine Testung auf den Einfluss des stetigen Rohwertes des MaastrichtFragebogens jeweils in den Einzelphasen auf die HR und die HRV-Parameter im Rahmen einer einfachen linearen Regression durchgeführt. Die HR und die HRV-Parameter wurden jeweils einzeln und jeweils nur in einer Phase als abhängige Parameter und der stetige Wert der Vitalen Erschöpfung als Prädiktor eingefügt. Die Ergebnisse sind in Tabelle 12 aufgeführt.

\section{Gehphase Taktatmungsphase Ruhephase}

\begin{tabular}{ll}
\hline mittlere HR $<0,05^{\star}($ Beta: $-0,13)$ & \\
\hline Ln SDNN & \\
\hline Ln rMSSD & \\
\hline pNN50 & $<0,05^{*}($ Beta: 0,10$)$ \\
\hline Ln LF & \\
\hline Ln HF & $<0,001^{* \star *}\left(\right.$ Beta:-0,17) $<0,001^{* * *}($ Beta: $-0,20)$ \\
\hline Ln Total
\end{tabular}

Tabelle 12 Einfache lineare Regression mit dem Prädiktor stetige Werte für „Vitale Erschöpfung“ und jeweils HR beziehungsweise einem HRV-Parameter aus einer Phase als abhängigem Parameter. Angegeben sind nur signifikante Effekte mit $\mathbf{p}-$ Wert und Signifikanzniveau $(\mathbf{p}<0,05=* ; \mathbf{p}<0,01=* * ; \mathbf{p}<0,001=* * *)$ und den dazugehörigen Beta-Werten.

Für mittlere HR zeigte sich ein signifikanter inverser Zusammenhang in der Gehphase. Bei höheren Werten für Vitale Erschöpfung war die mittlere HR in der Gehphase niedriger (s. Tabelle 12). Ln HF zeigte einen signifikanten konkordanten Zusammenhang in der Ruhephase (s. Tabelle 12). Je erschöpfter der Proband war, desto höher war der Wert für Ln HF. Für Ln LF/HF zeigte sich ein signifikanter inverser Zusammenhang mit dem Ausmaß der Erschöpfung für Taktatmungs- und Ruhephase (s. Tabelle 12). Je erschöpfter der Proband war, desto niedriger war der Wert für Ln LF/HF. 
Die beobachteten Effekte entsprechen nicht der vermuteten Sympathikusdominanz bei vital erschöpften Probanden.

Im Rahmen der Gehphase unter körperlicher Belastung geht ein starkes Ausmaß Vitaler Erschöpfung mit signifikant erniedrigter mittlerer HR einher. Hier scheint es sich analog zu den Veränderungen, die für die Depressiven für mittlere HR in der Gehphase beobachtet wurden, um eine geringere Motivation im Rahmen der Gehphase und damit geringerer körperlicher Aktivität entsprechend einer geringeren Aktivierung des Sympathikus bei den Vital Erschöpften zu handeln.

Im Rahmen der Taktatmungsphase war ein Anstieg der Ln LF erwartet worden (s. 1.3.3.2 Atmung, S.10). Es müsste sich dann auch der Quotient Ln LF/HF bei steigendem LF im Rahmen der Taktatmung erhöhen. Die erniedrigten Ln LF/HF-Werte bei zunehmendem Ausmaß der Vitalen Erschöpfung könnten durch eine geringe Resonanzfähigkeit, aber wohl eher durch eine mangelnde Mitarbeit im Rahmen der Taktatmungsphase begründet sein. Dieser Zusammenhang von erniedrigtem Ln LF/HF in der Taktatmungsphase mit zunehmender psychischer Belastung zeigte sich nicht nur für zunehmende Vitale Erschöpfung sondern auch für vorhandene Depressivität im Rahmen der Hypothese 1 (s. Tabelle 10 (S.44), s. Abb. 2 (S.45)). Auch die hohen Werte für Ln HF und die erniedrigten Werte für Ln LF/HF in der Ruhephase bei hohem Ausmaß der Vitalen Erschöpfung sind nicht hypothesenkonform. Hier zeigt sich mit zunehmendem Ausmaß Vitaler Erschöpfung keine Sympathikusdominanz oder Vagussuppression. Für Ln LF/HF zeigt sich wiederum eine Analogie zu den Ergebnissen für Depressivität. Auch hier war bei zunehmender psychischer Belastung eine Erniedrigung der Ln LF/HF in der Ruhephase beobachtet worden (s. Tabelle 10 (S.44), s. Abb. 2 (S.45)).

Unterhypothese 2 A zu Vitaler Erschöpfung muss verworfen werden.

\subsection{Unterhypothese B}

Um zu prüfen, ob der in Hypothese 2A für Vitale Erschöpfung beobachtete signifikante Effekt für mittlere HR in der Gehphase, Ln HF in der Ruhephase und Ln LF/HF in der Taktatmungs- und Ruhephase (s.Tab.12, S.51) in einem Modell mit somatischen Einflussfaktoren erhalten bleibt, wird ein neues Modell mit diesen Parametern formuliert. 
In einem Allgemeinen Linearen Modell werden zunächst die Werte der HR beziehungsweise der HRV-Parameter als abhängige Parameter in den Phasen angegeben, in denen sie in Hypothese 2 A einen signifikanten Unterschied zeigten.

Primär wird als stetiger Prädiktor „Vitale Erschöpfung“ angegeben. Im Anschluss werden einzeln einige somatische Faktoren in das Modell integriert. Diese sind: Alter, Geschlecht, BMI, Vorerkrankung HI, KHK, DM, HTN, Z.n. MI, pAVK, cerebrovaskuläre Vorerkrankung, Rauchstatus, Alkoholmenge pro Woche, Vormedikation Betablocker beziehungsweise Antiarrhythmika, LVEF, diastolischer Herzinsuffizienzscore, Uhrzeit der Langzeit-EKGTestung, Länge der Gehteststrecke und Höhe des logarithmierten NT-pro-BNP.

Im Weiteren werden lediglich Confounder/ Mediatoren aufgelistet (wenn ein Parameter einen signifikanten Effekt zeigt, die Vitale Erschöpfung dagegen keinen unabhängigen Effekt aufweist) beziehungsweise die Parameter, die neben dem psychischen Parameter einen unabhängigen Effekt haben (beide zeigen einen signifikanten Zusammenhang).

\subsection{Mittlere HR}

Als mögliche Mediatoren oder Confounder des Effekts der Vitalen Erschöpfung auf die mittlere HR in der Gehphase zeigten sich im Rahmen des unter 3.2.2.2.2 Unterhypothese B (S.52) beschriebenen Allgemeinen Linearen Modells die Länge der Gehstrecke, die Vormedikation mit Betablocker und die Höhe des Wertes für Ln NT-pro-BNP.

Einen jeweils unabhängigen Einfluss auf die mittlere HR in der Gehphase im Rahmen des Allgemeinen Linearen Modells hatten zusätzlich zur Vitalen Erschöpfung die jeweils einzeln neben „Vitaler Erschöpfung“ eingefügten Parameter Alter, Z. n. MI, Vormedikation mit Antiarrhythmika, KHK, HTN, PAVK und diastolischer Herzinsuffizienzscore. Hier zeigten dann im Modell sowohl Vitale Erschöpfung als auch der andere eingefügte Parameter einen signifikanten Einfluss auf die mittlere HR.

\subsection{Ln HF}

Für die Ruhephase zeigte sich in der Testung im Allgemeinen Linearen Modell ein möglicher Mediator- beziehungsweise Confoundereffekt von Geschlecht, der Vormedikation mit Betablocker und der Höhe des Ln NT-pro-BNP. Hier war der Effekt der Vitalen Erschöpfung auf die Ln HF jeweils neben dem einzeln zusätzlich eingefügten Parameter nicht mehr signifikant, der Effekt des neuen somatischen Parameters allerdings deutlich signifikant. 
Einen jeweils unabhängigen Einfluss im Rahmen des unter 3.2.2.2.2 Unterhypothese B (S.52) beschriebenen Allgemeinen Linearen Modells hatten auf die Ln HF in der Ruhephase die folgenden einzeln zusätzlich zur Vitalen Erschöpfung in das Modell integrierten Parameter: Vorerkrankung DM und diastolischer Herzinsuffizienzscore. Hier zeigte jeweils der eingefügte Parameter neben der ebenfalls signifikanten Vitalen Erschöpfung einen signifikanten Einfluss auf Ln HF für die Ruhephase.

\subsection{Ln LF/HF}

Als mögliche Mediatoren oder Confounder des Effekts der Vitalen Erschöpfung auf die Ln LF/HF zeigte sich im Rahmen des unter 3.2.2.2.2 Unterhypothese B (S.52) beschriebenen Allgemeinen Linearen Modells für die Taktatmungs-beziehungsweise Ruhephase keiner der einzeln eingefügten somatischen Parameter.

Einen jeweils unabhängigen Einfluss zeigten im Allgemeinen Linearen Modell in der Taktatmungsphase neben der Vitalen Erschöpfung die einzeln eingefügten Parameter Alter, KHK, pAVK, Vormedikation mit Betablockern, Vormedikation mit Antiarrhythmika, LVEF, Werte im diastolischen Herzinsuffizienzscore, Gehteststreckenlänge und Höhe des Ln NT-pro-BNP. In der Ruhephase Alter, Vorerkrankung HI, KHK, Vormedikation mit Betablocker, Antiarrhythmika, Wert im diastolischen Herzinsuffizienzscore, Gehteststreckenlänge und Höhe des Ln NT-pro-BNP. Hier war jeweils für den stetigen Wert der Vitalen Erschöpfung und für den einzeln eingefügten somatischen Parameter und die ausgewählte Phase ein signifikanter Einfluss der beiden Parameter auf Ln LF/HF.

Die Vormedikation mit Betablocker, die Gehteststreckenlänge und die Höhe des Ln NT-proBNP zeigte einen Mediator- beziehungsweise Confoundereffekt auf die mittlerer HR in der Gehphase. Diese Phase war im Rahmen der Prüfung der Unterhypothese 2 A (s.Tab.12, S.51) als Phase identifiziert worden, in der für Vitale Erschöpfung ein signifikanter Einfluss auf die mittlere HR besteht.

Das Geschlecht, die Höhe des NT-pro-BNP und die Vormedikation mit Betablocker zeigten für Ln HF in der Ruhephase einen Mediator-beziehungsweise Confoundereffekt. Diese Phase war im Rahmen der Prüfung der Unterhypothese 2 A (S.51) für Vitale Erschöpfung als Phase identifiziert worden, in der Vitale Erschöpfung einen signifikanten Einfluss auf die Ln HF hat. 
Viele der somatischen Faktoren und der kardialen Schweregradmarker zeigten einen unabhängigen Einfluss neben der Vitalen Erschöpfung auf die mittlere HR, Ln HF und Ln LF/HF. Für diese Faktoren ist ein Teil des Effekts der Vitalen Erschöpfung unabhängig von den somatischen Variablen.

Unterhypothese 2B für Vitale Erschöpfung muss für mittlere HR für Gehteststreckenlänge, Höhe des Ln NT-pro-BNP und die Vormedikation mit Betablocker verworfen werden. Für Ln HF muss Unterhypothese B für das Geschlecht, die Höhe des Ln NT-pro-BNP und die Vormedikation Betablocker verworfen werden. Von anderen kardialen Erkrankungen und kardialen Schwergradmarkern erscheint der Effekt der Vitalen Erschöpung zumindest teilweise unabhängig.

\subsection{Unterhypothese C}

Erwartet worden war, dass der Effekt der Vitalen Erschöpfung auf die HR und die HRVParameter besonders deutlich in der Taktatmungsphase nachzuvollziehen ist. Dies ließ sich im Rahmen der Testung unter 3.2.2.2.1 Unterhypothese A (s.Tab.12, S.51) nicht nachweisen. Unterhypothese $\mathrm{C}$ muss daher abgelehnt werden.

\subsection{Unterhypothese D}

Die in Hypothese 1A beschriebenen Effekte der Vitalen Erschöpfung auf die HR- und HRVParameter wurden sowohl für Männer als auch für Frauen erwartet.

Es wurde eine lineare Regression wie unter 3.2.2.2.1 Hypothese A beschrieben für die in Tabelle 12 (S.51) aufgeführten signifikante Effekte durchgeführt mit Selektion für nur Männer beziehungsweise nur Frauen.

Für mittlere HR zeigte sich für die Gesamtkohorte und für Frauen ein signifikanter inverser Zusammenhang zwischen Vitaler Erschöpfung und mittlerer HR in der Gehphase. Für Männer zeigte sich kein signifikanter Effekt.

Für Ln HF zeigte sich nur für die Gesamtkohorte ein signifikanter Effekt der Vitalen Erschöpfung auf die Ln HF in der Ruhephase, in der geschlechtergetrennten Analyse war weder für Frauen noch für Männer ein Effekt nachweisbar.

Für die Ln LF/HF zeigte sich sowohl für die Taktatmungs- als auch die Ruhephase ein signifikanter inverser Effekt für die Gesamtkohorte und die Frauen. Jeweils kein Effekt der Vitalen Erschöpfung war auf die Ln LF/HF für Männer nachweisbar. 
Unterhypothese $2 \mathrm{D}$ zu Vitaler Erschöpfung muss abgelehnt werden, da sich bei geschlechtergetrennter Auswertung die in Hypothese 2 A für Vitale Erschöpfung beschriebenen nicht hypothesenkonformen signifikanten Effekte teilweise nicht oder nur für Frauen nachweisen lieBen.

Zusammenfassend zeigte sich für Vitale Erschöpfung ein signifikanter Effekt für mittlere HR, Ln HF und Ln LF/HF in einzelnen Phasen, allerdings keine Sympathikusdominanz oder Vagussuppression. Diese signifikanten Haupteffekte waren nur teilweise unabhängig vom somatischen Status (B), in der Taktatmungsphase nicht besonders ausgeprägt (C) und ließen sich in der geschlechtergetrennten Betrachtung nur teilweise nachweisen (D).

\section{Hypothese 2 zu Vitaler Erschöpfung muss daher abgelehnt werden.}

\subsubsection{Körperliche Funktionsfähigkeit des SF-36}

\subsection{Unterhypothese A}

Bezüglich körperlicher Funktionsfähigkeit zeigte sich in der primär durchgeführten Analyse mit dichotomisierten Parametern im Rahmen eines Allgemeinen Linearen Modells mit Messwiederholungen ein Haupteffekt für die mittlere HR und ein Interaktionseffekt für die mittlere HR und Ln LF/HF.

Um auch für körperliche Funktionsfähigkeit den Einfluss der stetigen Werte auf die HR und die HRV-Parameter zu ermitteln, wurde eine Testung auf den Einfluss der stetigen Werte der körperlichen Funktionsfähigkeit auf die einzelne HR- und HRV-Parametern, nach Untersuchungsphasen aufgetrennt, im Rahmen einer einfachen linearen Regression durchgeführt. Die HR und die HRV-Parameter wurden jeweils einzeln und nur für eine Phase als abhängiger Parameter und der stetige Wert der körperlichen Funktionsfähigkeit als Prädiktor eingefügt. Die Ergebnisse sind in Tabelle 13 auf der folgenden Seite aufgeführt. 


\begin{tabular}{llll}
\hline mittlere HR & $<0,001^{* * *}($ Beta: 0,24$)$ & & \\
\hline Ln SDNN & $<0,05^{*}($ Beta: 0,10$)$ & \\
\hline Ln rMSSD & $<0,001^{* * *}($ Beta: $-0,17)$ & & \\
\hline pNN50 & & $<0,001^{* * *}($ Beta: 0,25$)$ & $<0,001^{* * *}($ Beta: 0,22$)$ \\
\hline Ln LF & & \\
\hline Ln HF & $<0,01^{* *}($ Beta: $-0,16)$ & & \\
\hline Ln Total & & $<0,01^{* *}($ Beta: 0,14$)$ & $<0,001^{* *}($ Beta: 0,15$)$ \\
\hline Ln LF/HF & $<0,001^{* * *}($ Beta: 0,20$)$ & $<0,001^{* * *}($ Beta: 0,28$)$ & $<0,001^{* * *}(0,27)$ \\
\hline
\end{tabular}

Tabelle 13 Einfache lineare Regression mit den stetigen Werten der körperlichen Funktionsfähigkeit als Prädiktor und jeweils HR beziehungsweise einem HRV-Parameter aus einer Phase als abhängigem Parameter. Angegeben sind nur signifikante Effekte mit p-Wert und Signifikanzniveau $(\mathbf{p}<0,05=* ; p<0,01=* *$; $\mathbf{p}<0,001=* * *)$ und den dazugehörigen Beta-Werten.

Für die mittlere HR war ein signifikanter konkordanter Zusammenhang in der Gehphase nachweisbar. Bei niedrigen Werten für körperliche Funktionsfähigkeit zeigten sich also niedrige Werte für die mittlere HR. Dies entspricht nicht dem hypothetisierten Zusammenhang zu schlecht empfundener körperlicher Lebensqualität mit erhöhter mittlerer HR (s. Tabelle 13).

Für Ln SDNN zeigte sich ein signifikanter, konkordanter Zusammenhang in der Taktatmungsphase. Die Probanden mit als niedrig empfundener körperlicher Funktionsfähigkeit haben in der Taktatmungsphase niedrigere Werte für Ln SDNN (s. Tab. 13). Dies entspricht dem in der Hypothese vermuteten Zusammenhang von niedrig empfundener körperlicher Funktionsfähigkeit mit niedrigen Werten für Ln SDNN.

Für Ln rMSSD war für die Gehphase ein signifikanter inverser Zusammenhang erkennbar. Bei schlechter körperlicher Funktionsfähigkeit zeigte sich eine erhöhte Ln rMSSD. Dies entspricht nicht der Hypothese (s. Tabelle 13).

Für Ln LF zeigte sich ein signifikanter konkordanter Zusammenhang für Taktatmungs- und Ruhephase. Eine schlecht empfundene körperliche Funktionsfähigkeit geht also mit niedrigen Werten der Ln LF einher. Hier geht eine belastete Situation wie in der Hypothese vermutet mit niedrigen HRV-Werten einher (s. Tabelle 13).

Für Ln HF ließ sich ein signifikanter inverser Zusammenhang in der Gehphase erkennen (s. Tab.13). Hier war eine niedrige körperliche Funktionsfähigkeit mit hohen Werten des Ln HF verbunden. Dies entspricht nicht der Hypothese. 
Für Ln Total zeigte sich für Taktatmungs- und Ruhephase jeweils ein signifikanter konkordanter Zusammenhang mit körperlicher Funktionsfähigkeit (s.Tab.13, S.57). Niedrige Werte der körperlichen Funktionsfähigkeit gehen mit niedrigen Werten für Ln Total einher. Dies entspricht dem vermuteten Zusammenhang.

Für Ln LF/HF war ein signifikanter konkordanter Zusammenhang für alle drei Phasen nachweisbar (s.Tab.13, S.57). Niedrige körperliche Funktionsfähigkeit geht also mit niedrigen Werten einher. Die in den Hypothesen vermutete Sympathikusdominanz bei psychisch belasteten Probanden würde bei diesem Parameter allerdings einer Erhöhung bei Belastung entsprechen (TASK FORCE OF THE EUROPEAN SOCIETY OF CARDIOLOGY AND THE NORTH AMERICAN SOCIETY OF PACING AND ELECTROPHYSIOLOGY 1996), also einem inversen Zusammenhang. Daher entspricht dieser signifikante Zusammenhang nicht der Hypothese.

Die in der Gehphase signifikanten Zusammenhänge zwischen körperlicher Funktionsfähigkeit und mittlerer HR, Ln rMSSD, Ln HF und Ln LF/HF sind vermutlich mit vorhandener körperlicher Einschränkung verbunden. Damit scheint eine geringere Aktivität im Rahmen des Gehtestes und damit fehlender Aktivierung des Sympathikus sowie somatische Vorerkrankungen die wahrscheinlichste Erklärung für die vorhandenen Veränderungen zu sein. Für diese Parameter und LF/HF in der Taktatmungs- und Ruhephase wird, da sie nicht der Hypothese entsprechen, auf eine weitere Analyse im Rahmen der Unterhypothesen verzichtet.

Für Ln SDNN in der Taktatmungsphase und für Ln LF sowie Ln Total für Taktatmungs- und Ruhephase zeigte sich eine Absenkung bei zunehmend empfundener körperlicher Einschränkung. Für diese Parameter kann Unterhypothese A angenommen werden.

\subsection{Unterhypothese B}

Es wurde ein Allgemeines Lineares Modell für kategorielle und stetige Prädiktoren für die hypothesenkonformen Effekte von Ln SDNN, Ln LF und Ln Total jeweils in den Phasen gerechnet, in denen ein signifikanter Effekt im Rahmen der Unterhypothese A nachzuweisen war. Dafür wurden die HRV-Parameter in der jeweiligen Phase als abhängige Parameter und der stetige Parameter der körperlichen Funktionsfähigkeit als Prädiktor eingegeben.

Primär wird als stetiger Prädiktor „körperliche Funktionsfähigkeit“ angegeben. Im Anschluss werden einzeln einige somatische Faktoren in das Modell integriert. Diese sind: Alter, Geschlecht, BMI, Vorerkrankung HI, KHK, DM, HTN, Z.n. MI, pAVK, cerebrovaskuläre Vor- 
erkrankung, Rauchstatus, Alkoholmenge pro Woche, Vormedikation Betablocker beziehungsweise Antiarrhytmika, LVEF, diastolischer Herzinsuffizienzscore, Uhrzeit der LangzeitEKG-Testung, Länge der Gehteststreck und Höhe des logarithmierten NT-pro-BNP.

Im Weiteren werden lediglich Confounder/Mediatoren aufgelistet (wenn ein Parameter einen signifikanten Effekt zeigt, „körperliche Funktionsfähigkeit“ dagegen keinen unabhängigen Effekt aufweist) beziehungsweise die Parameter, die neben dem psychischen Parameter einen unabhängigen Effekt haben (beide zeigen einen signifikanten Zusammenhang).

\subsection{Ln SDNN}

Als möglicher Mediator oder Confounder des Effekts der körperlichen Funktionsfähigkeit auf die Ln SDNN in der Taktatmungsphase zeigte sich lediglich der BMI. Hier war der Effekt der körperlichen Funktionsfähigkeit auf die Ln SDNN im Rahmen der Analyse nicht mehr signifikant, der Effekt des BMI allerdings deutlich signifikant.

Einen jeweils unabhängigen Einfluss auf die Ln SDNN in der Taktatmungsphase hatten zusätzlich zur körperlichen Funktionsfähigkeit das Geschlecht und die Vorerkrankung DM.

\subsection{Ln LF}

Als mögliche Mediatoren oder Confounder des Effekts der körperlichen Funktionsfähigkeit auf die Ln LF in Taktatmungs- und Ruhephase zeigten sich keine der untersuchten somatischen Parameter.

Einen jeweils unabhängigen Einfluss auf die Ln LF neben körperlicher Funktionsfähigkeit im Allgemeinen Linearen Modell hatten die jeweils einzeln eingefügten Parameter Alter, Geschlecht, BMI, Vorerkrankung DM in der Taktatmungsphase und Alter, Vorerkrankung DM in der Ruhephase.

\subsection{Ln Total}

Als mögliche Mediatoren oder Confounder des Effekts der körperlichen Funktionsfähigkeit auf Ln Total zeigten sich in Taktatmungs- und Ruhephase keine der untersuchten Parameter.

Einen jeweils unabhängigen Einfluss auf die Ln Total neben der körperlichen Funktionsfähigkeit im Allgemeinen Linearen Modell hatten die jeweils einzeln eingefügten Parameter Geschlecht, Vorerkrankung DM in der Taktatmungsphase, in der Ruhephase die beiden vorgenannten somatischen Parameter und das Alter. 
Lediglich der BMI zeigte einen möglichen Mediator- beziehungsweise Confoundereffekt auf den Effekt der körperlichen Funktionsfähigkeit auf die Ln SDNN in der Taktatmungsphase. Die Taktatmungsphase war im Rahmen der Unterhypothese A (s. Tab. 13, S.57) als Phase identifiziert worden, in der für körperliche Funktionsfähigkeit ein signifikanter Einfluss auf die Ln SDNN besteht. Für den Effekt der körperlichen Funktionsfähigkeit auf Ln LF und Ln Total in Taktatmungs- und Ruhephase (s. Tab. 13, S.57) ließ sich kein Mediatoren/Confounder unter den untersuchten somatischen Parametern finden.

Viele der somatischen Faktoren und der kardialen Schweregradmarker zeigten einen unabhängigen Einfluss neben der körperlichen Funktionsfähigkeit auf die Ln SDNN, die Ln LF und Ln Total. Für diese Faktoren ist ein Teil des hypothesenkonformen Effekts der körperlichen Funktionsfähigkeit unabhängig von den somatischen Variablen.

Unterhypothese B für körperliche Funktionsfähigkeit muss für Ln SDNN für BMI verworfen werden. Von den übrigen untersuchten kardialen Erkrankungen und kardialen Schwergradmarkern erscheint der beobachtete Effekt der körperlichen Funktionsfähigkeit auf die in Unterhypothese B näher untersuchten HRV-Parameter Ln SDNN, Ln LF und Ln Total zumindest teilweise unabhängig zu sein.

\subsection{Unterhypothese C}

Erwartet worden war, dass der Effekt einer Einschränkung der körperlichen Lebensqualität auf die HR und die HRV-Parameter insbesondere in der Taktatmungsphase zu beobachten ist. Für Ln LF und Ln Total zeigte sich ein signifikanter Effekt in der Taktatmungsphase für körperliche Funktionsfähigkeit im Rahmen der linearen Regression in Unterhypothese A (s. Tab. 13, S.57), der aber nicht besonders deutlich in der Taktatmungsphase ausfiel.

Für Ln SDNN zeigte sich der einzige signifikante und hypothesenkonforme Zusammenhang in der Taktatmungsphase (s. Tab. 13, S.57).

Der hypothesenkonforme Effekt der körperlichen Funktionsfähigkeit auf die Ln SDNN war ausschließlich in der Taktatmungsphase nachweisbar. Für diesen Parameter kann Unterhypothese $\mathrm{C}$ angenommen werden. Für alle weiteren muss sie abgelehnt werden. 


\subsection{Unterhypothese D}

Die in Hypothese 2A zu körperlicher Funktionsfähigkeit beschriebenen signifikanten hypothesenkonformen Effekte (s. Tab.13, S.57) wurden sowohl für Männer als auch für Frauen erwartet.

Es wurde analog der linearen Regression beschrieben unter 3.2.2.3.1 Unterhypothese A (S.57) eine Analyse für die signifikanten Effekte durchgeführt mit Selektion für nur Männer beziehungsweise nur Frauen.

Für Ln SDNN zeigte sich in der Taktatmungsphase ein signifikanter Effekt. Für Männer war dieser Effekt weiterhin nachzuvollziehen. Für Frauen nicht.

Für die Ln LF ließ sich für die Gesamtkohorte, Männer und Frauen jeweils ein signifikanter Effekt der körperlichen Funktionsfähigkeit in Taktatmungs- und Ruhephase nachweisen.

Für Ln Total zeigte sich, jeweils getrennt betrachtet nach Taktatmungs- und Ruhephase, für Gesamtkohorte und Männer ein signifikanter Effekt, für Frauen nicht.

Unterhypothese D für körperliche Funktionsfähigkeit muss für Ln SDNN und Ln Total jeweils in der Taktatmungs- und Ruhephase abgelehnt werden, da sich bei geschlechtsgetrennter Auswertung der Effekt, der in der Gesamtkohorte nachweisbar war, nur für Männer nachweisen ließ. Für Ln LF in Taktatmungs- und Ruhephase kann Unterhypothese D angenommen werden.

In der Gehphase zeigten sich einige nicht hypothesenkonforme Zusammenhänge im Rahmen der Unterhypothese A, die am ehesten ein Zeichen für bei eingeschränkter körperlicher Funktionsfähigkeit vorhandene somatische Erkrankungen ist und damit in der Gehphase eine verminderte körperliche Aktivität mit niedriger Sympathikusaktivierung illustriert. Auch LF/HF in Taktatmungs- und Ruhephase weist einen nicht hypothesenkonformen Effekt auf. Daraufwurde nicht näher eingegangen.

Hypothesenkonforme Zusammenhänge zeigten sich für Ln SDNN, Ln LF und Ln Total.

Die körperliche Funktionsfähigkeit hatte lediglich in der Taktatmungsphase einen deutlichen hypothesenkonformen signifikanten Effekt auf die Ln SDNN, wobei sich für BMI ein Mediator- beziehungsweise Confoundereffekt zeigte, aber sonst der Effekt zumindest teilweise unabhängig von somatischen Erkrankungen zu sein scheint. 
Für Ln LF und Ln Total zeigte sich ein hypothesenkonformer Effekt der körperlichen Funktionsfähigkeit in etwa gleichem Maße in der Taktatmungs- und in der Ruhephase. Für Ln LF und Ln Total zeigte sich kein somatischer Faktor als Mediator beziehungsweise Confounder. Zumindest teilweise kann also von einer Unabhängigkeit des Effektes der körperlichen Funktionsfähigkeit auf Ln LF und Ln Total ausgegangen werden. In der geschlechtergetrennten Betrachtung war der Effekt der körperlichen Funktionsfähigkeit für Ln Total nicht stabil.

Damit können für Ln SDNN Unterhypothese A und C, sowie B teilweise angenommen werden, Unterhypothese D muss abgelehnt werden.

Für Ln LF und Ln Total können Unterhypothese A und B angenommen werden. Unterhypothese C muss für beide Parameter abgelehnt werden. Unterhypothese D kann für Ln LF angenommen werden.

Hypopothese 2 für körperliche Funktionsfähigkeit kann für einzelne HRV-Parameter teilweise angenommen werden.

\subsubsection{Zusammenhang zwischen kardialer Vorerkrankung, kardialen Schweregradmarkern und HR sowie HRV-Parametern (Hypothese 3)}

Angenommen worden war, dass insbesondere die hochfrequenten, vagalen (rMSSD, pNN50, HF) HRV-Werte klare Zusammenhänge zu dem Ausmaß der Vorerkrankungen und den objektiven kardialen Parametern zeigen. Bei Zeichen starker Vorerkrankung oder Schädigung wird eine niedrige HRV erwartet.

Zur Prüfung dieser Hypothese wird die Korrelation der HR und der HRV-Parameter mit den physischen Vorerkrankungen und den objektiven Parametern (diastolischer Herzinsuffizienzscore, LVEF, NT-pro-BNP, Gehstrecke, BMI, Alter) in einer Korrelationsmatrix berechnet. Für die dichotomen und kategoriellen Parameter wird eine non-parameterische Korrelationsmatrix nach Kendalls Tau gerechnet (s. Tabellen 14 und 15 auf den folgenden Seiten). 


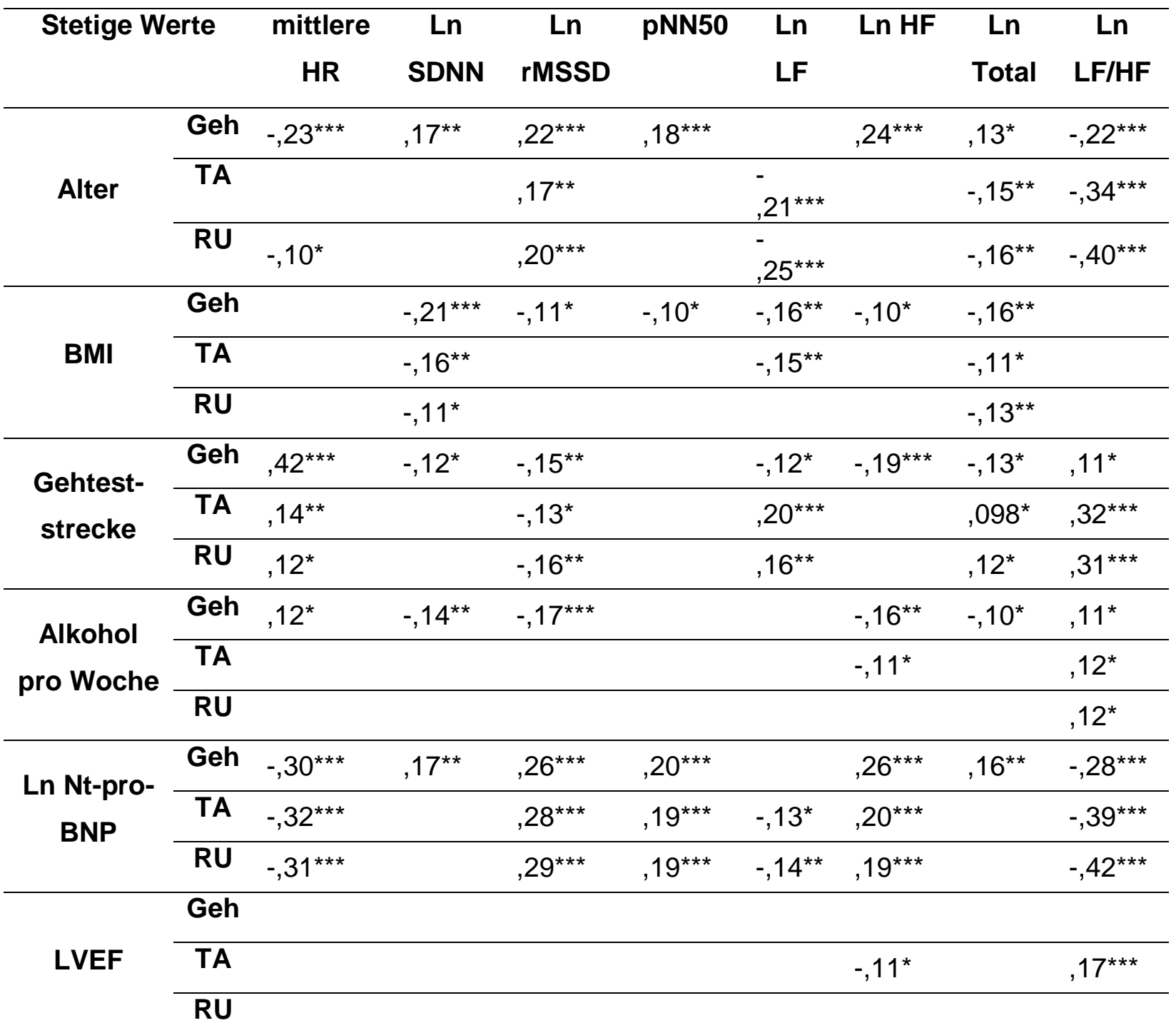

Tabelle 14 rechteckige Korrelationsmatrix. 1. Variablenliste HR/HRV-Parameter der jeweiligen Phase, 2. Variablenliste stetige kardiale Risikofaktoren beziehungsweise objektive Parameter. Lediglich der Korrelationskoeffizient $\mathbf{r}$ ist aufgezeigt. Nur signifikante Zusammenhänge sind abgebildet und die Höhe der Signifikanz durch Sterne gekennzeichnet. $\mathbf{p}<0,05^{*}, \mathbf{p}<0,01 * *, \mathbf{p}<0,001 * * *$. Es wurde fallweise ausgeschlossen aufgrund von fehlenden Daten.

\begin{tabular}{|c|c|c|c|c|c|c|c|c|}
\hline $\begin{array}{c}\text { Dichtome und } \\
\text { kategorielle } \\
\text { Parameter }\end{array}$ & & $\begin{array}{c}\text { mittlere } \\
\text { HR }\end{array}$ & $\begin{array}{c}\text { Ln } \\
\text { SDNN }\end{array}$ & $\begin{array}{c}\text { Ln } \\
\text { rMSSD }\end{array}$ & pNN50 Ln LF & $\begin{array}{l}\text { Ln } \\
\text { HF }\end{array}$ & $\begin{array}{c}\text { Ln } \\
\text { Total }\end{array}$ & $\begin{array}{c}\text { Ln } \\
\text { LF/HF }\end{array}$ \\
\hline \multirow{3}{*}{ Geschlecht } & Geh & & 0,07 & 0,10 & 0,08 & 0,08 & & $-0,08$ \\
\hline & TA & & 0,07 & 0,06 & & 0,08 & 0,07 & \\
\hline & RU & & & & & 0,07 & & \\
\hline \multirow{3}{*}{ KHK } & Geh & $-0,22$ & & & & & 0,09 & \\
\hline & TA & $-0,15$ & & 0,08 & 0,07 & & & $-0,11$ \\
\hline & $\mathbf{R U}$ & $-0,14$ & & 0,07 & & 0,07 & & $-0,11$ \\
\hline
\end{tabular}




\begin{tabular}{|c|c|c|c|c|c|c|c|c|c|}
\hline & Geh & $-0,13$ & & & & & & & \\
\hline \multirow[t]{3}{*}{ Z.n. MI } & TA & $-0,11$ & & & & & & & \\
\hline & RU & $-0,10$ & & & & & & & \\
\hline & Geh & $-0,07$ & & & & & & & \\
\hline \multirow[t]{3}{*}{ pAVK } & TA & & & & & & 0,07 & & $-0,09$ \\
\hline & RU & & & & & & & & \\
\hline & Geh & & $-0,08$ & & & $-0,10$ & & & \\
\hline \multirow[t]{3}{*}{ DM } & TA & 0,11 & $-0,10$ & & & $-0,13$ & & $-0,13$ & $-0,08$ \\
\hline & RU & 0,10 & $-0,10$ & & & $-0,11$ & & $-0,13$ & \\
\hline & Geh & $-0,11$ & & & & & & & \\
\hline \multirow[t]{3}{*}{ HTN } & TA & $-0,15$ & & & & & & & \\
\hline & RU & $-0,13$ & & & & & & & $-0,08$ \\
\hline & Geh & $-0,35$ & 0,11 & & & 0,09 & & 0,12 & \\
\hline \multirow[t]{3}{*}{ Betablocker } & TA & $-0,41$ & & & & & 0,11 & & $-0,16$ \\
\hline & RU & $-0,40$ & & 0,10 & & & 0,09 & & $-0,16$ \\
\hline & Geh & $-0,10$ & & & & & & & \\
\hline \multirow[t]{2}{*}{ Antiarrhythmika } & TA & $-0,09$ & & & & & 0,07 & & $-0,10$ \\
\hline & $\mathbf{R U}$ & $-0,10$ & & & & & & & $-0,09$ \\
\hline \multirow{3}{*}{$\begin{array}{l}\text { diastolischer } \\
\text { Herzinsuffizienz- } \\
\text { score }\end{array}$} & Geh & $-0,15$ & & 0,07 & & & 0,08 & 0,07 & $-0,08$ \\
\hline & TA & $-0,11$ & & 0,07 & & & & & $-0,10$ \\
\hline & RU & $-0,11$ & & 0,12 & 0,10 & & 0,10 & & $-0,14$ \\
\hline \multirow{3}{*}{$\mathrm{HI}$} & Geh & $-0,07$ & & 0,09 & 0,08 & & 0,12 & 0,07 & $-0,14$ \\
\hline & TA & & & & & & & & $-0,06$ \\
\hline & RU & & & 0,09 & 0,07 & & & & $-0,12$ \\
\hline
\end{tabular}

Tabelle 15 Korrelationsmatrix nach Kendalls Tau für dichotome und kategorielle Faktoren. Variablenliste: HR und HRV-Parameter der einzelnen Phasen sowie dichotome und kategorielle somatische Parameter. Aufgeführt sind lediglich signifikante Zusammenhänge ab einem 5\% - Signifikanzniveau. Die verwendete Kodierung war 1 für Krankheit vorhanden, 0 nicht vorhanden. Männer waren mit 1, Frauen mit 2 kodiert. Der diastolische Herzinsuffizienzscore war von 0 bis 3 entsprechend zunehmender Schwere der diastolischen Dysfunktion kodiert.

Für die Ln rMSSD wurde als Zeichen von Schädigung bei vorhandener Vorerkrankung oder objektiv höherer somatischer Krankheitsschwere eine Erniedrigung erwartet. Nur in der Gehphase zeigte sich eine Erniedrigung für zunehmenden BMI und steigenden Alkoholkonsum. 
Für die PNN50 wurde als Zeichen von Schädigung bei vorhandener Vorerkrankung oder objektiv höherer somatischer Krankheitsschwere eine Erniedrigung erwartet. Lediglich in der Gehphase zeigte sich eine Erniedrigung für zunehmenden BMI.

Für die Ln HF wurde als Zeichen von Schädigung bei vorhandener Vorerkrankung oder objektiv höherer somatischer Krankheitsschwere eine Erniedrigung erwartet. In der Gehphase zeigte sich eine Erniedrigung für zunehmenden BMI und Alkohol. In der Taktatmungsphase zeigte sich eine Erniedrigung für zunehmenden Alkoholkonsum. In der Ruhephase kein $\mathrm{Zu}-$ sammenhang in diese Richtung.

Ungewöhnlich war, dass sich für kardiale Vorerkrankungen wie Herzinsuffizienz sowie für zunehmende laborchemische oder echokardiographische Herzinsuffizienzzeichen ansteigende HRV-Parameter und erniedrigte HR-Werte nachweisen ließen. Dies ist für die vorgenannten Vorerkrankungen nicht zu erwarten, entspricht aber der Wirkung von Betablockern (s. 1.3.3.3 Medikamente und Genussmittel, S.11), die häufig bei kardialen Vorerkrankungen Bestandteil des Therapieschemas sind. Hier wurde, da es sich nicht um die primäre Fragestellung handelt, keine weitergehende Analyse angeschlossen.

Angenommen werden kann Hypothese 3 für erhöhten BMI und Alkoholkonsum. Dieser Zusammenhang ist hauptsächlich in der Gehphase nachzuvollziehen, allerdings nicht für alle vagalen Parameter.

\subsubsection{Zusammenhang zwischen 6-Minuten-Gehteststrecke und HR und HRV-Parametern (Hypothese 4)}

„Höhere HR und erniedrigte HRV-Werte in Ruhe sagen eine kürzere 6-Minuten-Gehstrecke voraus".

Getestet wird dies in einem multiplen Regressionsmodell. Die HR und die HRV-Parameter der Ruhephase werden als unabhängige Prädiktoren und die Gehstrecke als abhängige Variable angegeben. Gefordert wird für HR ein inverser Zusammenhang und für die HRV-Parameter ein konkordanter Zusammenhang. Es zeigte sich kein signifikanter Zusammenhang.

\section{Hypothese 4 muss verworfen werden.}




\subsubsection{Zusammenhang zwischen psychologischen Variablen und kardia- len Schweregradmarkern (Hypothese 5)}

„Kardiale Schweregradmarker korrelieren schwach mit Depressivität, Vitaler Erschöpfung und der körperlichen Funktionsfähigkeit der Lebensqualität, aber nicht mit Angst““.

Im Rahmen einer Korrelationsanalyse werden in der ersten Variablenliste die psychischen Variablen in stetiger Skalierung und in der zweiten Variablenliste die kardialen Schweregradmarker (Gehstrecke, NT-pro-BNP, LVEF) miteinander korreliert. Für den kategoriellen Parameter „diastolischer Herzinsuffzienzscore“ wurde ein nicht-parametrischer Korrelationstest nach Kendalls Tau gerechnet.

\begin{tabular}{ccccc}
\hline & Gehteststrecke & NT-pro-BNP & LVEF & $\begin{array}{l}\text { diastolischer } \\
\text { Herzinsuffizienzscore }\end{array}$ \\
\hline $\begin{array}{c}\text { körperliche } \\
\text { Funktionsfähigkeit }\end{array}$ & $0,56^{* * *}$ & $-0,29^{\star * *}$ & $0,15^{\star *}$ & $-0,14^{\star *}$ \\
\hline $\begin{array}{c}\text { Vitale } \\
\text { Erschöpfung }\end{array}$ & $-0,24^{* * *}$ & $0,13^{\star *}$ & \\
\hline Depressivität & $-0,12^{*}$ & & \\
\hline
\end{tabular}

\section{Angst}

Tabelle 16 rechteckige Korrelationsmatrix: 1. Variablenliste mit psychischen Variablen, 2. Variablenliste mit objektiven kardialen Schweregradmarkern. Die Stichprobengröße variiert aufgrund von fehlenden Daten zwischen 416 und 461. Aufgezeigt ist lediglich der Korrelationskoeffizient r, die Stärke der Signifikanz ist mit Sternen markiert. Der diastolische Herzinsuffzienzscore ist mit einem nicht-parametrischen Korrelationstest nach Kendalls Tau berechnet worden. Zu beachten ist, dass hohe Werte für körperliche Funktionsfähigkeit einer guten Lebensqualität entsprechen, steigende Werte der anderen psychologischen Variablen einer zunehmenden psychischen Belastung entsprechen.

Eine hohe körperliche Funktionsfähigkeit korreliert höchst signifikant mit langen Gehteststrecken, niedrigen Werten des NT-pro-BNP, guten Werten für LVEF und niedrigem diastolischem Herzinsuffzienzscore.

Je stärker vital erschöpft die Probanden waren, desto signifikant kürzer war die Gehteststrecke und desto signifikant höher die Werte für NT-pro-BNP.

Für Depressive zeigte sich eine signifikant kürzere Gehteststrecke.

Für Ängstliche zeigte sich kein signifikanter Zusammenhang. 
Hypothese 5 kann bezüglich der Gehteststrecke für alle untersuchten psychologischen Variablen angenommen werden. Bezüglich Ln NT-pro-BNP zeigte sich für körperliche Funktionsfähigkeit und Vitale Erschöpfung ein hypothesenkonformer Zusammenhang. Die LVEF und der diastolische Herzinsuffzienzscore zeigten nur mit körperlicher Funktionsfähigkeit eine signifikante Korrelation.

\section{Hypothese 5 kann für Vitale Erschöpfung und Depressivität teilweise angenommen werden.}

Hypothese 5 kann für Angst und körperliche Funktionsfähigkeit angenommen werden.

\subsubsection{Explorative Analysen}

\subsubsection{Nur unbearbeitete EKGs}

Aufgrund von Artefakten und anderen Ausschlusskriterien sind lediglich 423 Datensätze für Phase 1 vorhanden. Davon mussten bei 96 EKGs EKG-Zeilen im Rahmen der EKG-Analyse ausgeschlossen werden. Die Ausschlüsse betrafen bei 74 Probanden unter anderem die erste EKG-Zeile. Bei Ausschluss der ersten EKG-Zeile reduzierte sich die Abtastfrequenz (s. Tabelle 1). Eine T-Testung mit Gruppen berechnet mit getrennten Varianzen zeigte, dass die EKGs insbesondere mit Ausschluss der EKG-Zeile 1 sich signifikant von den Werten der unbearbeiteten EKGs unterschieden (s. 6.6 T-Testung bearbeitete und unbearbeitete EKGs, S. 125) Dies betrifft allerdings fast nur die Gehphase. In der Taktatmungsphase und in der Ruhephase waren lediglich in Einzelfällen (unter 3 Fällen) ein Ausschluss einzelner EGK-Zeilen notwendig.

Aufgrund der signifikanten Unterschiede zwischen den bearbeiteten und unbearbeiteten EKGs (s. Anhang 6.6 T-Testung bearbeitete und unbearbeitete EKGs, S.125) wurde eine explorative Analyse ausschließlich mit unbearbeiteten EKGs durchgeführt.

Untersucht wurden 327 EKGs, die keine Bearbeitung der EKG-Zeilen erfahren hatten, analog der Auswertungen von Hypothese 1 und 2.

Eine Repräsentativitätsprüfung der Kollektive bezüglich des Kriteriums des Bearbeitungsstatus in der Gehphase wurde anhand der unter Repräsentativitätsprüfung (2.5.3.1 Repräsentativitätsprüfung, S.34) aufgeführten Kriterien durchgeführt. Im Rahmen der Chi-QuadratTestung zeigte sich eine Abhängigkeit der Gruppenzuteilung vom Geschlecht. In der Gruppe der bearbeiteten EKGs waren unerwartet viele Frauen. Dies könnte an der größeren präthora- 
kalen Masse liegen, die während Bewegung zu Artefakten durch Mitbewegung der Elektroden auf der Haut führte. Dies wurde bereits während der Datenerhebung bemerkt, ließ sich aber nicht befriedigend methodisch verhindern.

Auch waren im t-Test die Werte der Angstskala in der Gruppe der unbearbeiteten EKGs signifikant niedriger als die in der bearbeiteten Gruppe. Hier könnte der Geschlechtsunterschied zwischen den Gruppen einen Mediatoreffekt haben.

\subsection{Depressivität (Hypothese 1)}

322 Werte für Depressivität (Depressiv/Nicht-Depressiv) anhand der HADS lagen zur Untersuchung unter Ausschluss der bearbeiteten EKGs vor. Von diesen 322 Fällen lagen aufgrund von Ausschlüssen teilweise keine vollständigen Datensätze bezüglich der HR und der HRVParameter vor. Daher konnten ca. 30 Fälle nicht untersucht werden. Es zeigte sich ein Haupteffekt für die mittlere Herzrate. Ein Interaktionseffekt zeigte sich bei mittlerer HR, Ln LF, Ln Total, Ln LF/HF.

Daraufhin wurden analog des Verfahrens in 3.2.1.1 Unterhypothese A (S.43) für diese signifikanten Effekte Testungen nach Untersuchungsphase aufgespalten gerechnet.

Hier zeigte sich für die Gehphase ein signifikanter Effekt für mittlere HR, Ln LF und Ln Total. Die Depressiven hatten in der Gehphase niedrigere Werte für mittlere HR und jeweils höhere Ln LF und Ln Total als die Vergleichsgruppe.

Für Taktatmungs- und Ruhephase zeigte sich ein signifikanter Effekt für Ln LF/HF. Hier waren die Mittelwerte der Depressiven jeweils niedriger als die der Vergleichsgruppe.

Die Bewertung erfolgt im Rahmen der Diskussion.

In der Analyse mit stetigen Werten der HADS-Depressivitätsskala zeigte sich wie in der Analyse unter 3.2.1.1 Unterhypothese A (S.43) kein Haupteffekt, es gab lediglich Interaktionseffekte.

\subsection{Angst (Hypothese 2)}

323 Werte für Angst (ängstlich/nicht-ängstlich) anhand der HADS lagen zur Untersuchung unter Ausschluss der bearbeiteten EKGs vor. Von diesen 323 Fällen lagen aufgrund von Ausschlüssen teilweise keine vollständigen Datensätze bezüglich der HR und der HRV-Parameter vor. Daher konnten ca. 30 Fälle nicht untersucht werden. Es zeigten sich keine Haupt-oder Interaktionseffekte. Auch in der Analyse mit stetigen Werten der HADS-Angstskala zeigten 
sich keine Haupt-oder Interaktionseffekte. Dies entspricht den Ergebnissen der Hypothese 2 zu Angst (s. 3.2.2.1 Angst, S.50).

\subsection{Vitale Erschöpfung (Hypothese 2)}

299 Werte für Vitale Erschöpfung lagen in dieser Analyse unter Ausschluss der bearbeiteten EKGs vor. Von diesen lagen aufgrund von Ausschlüssen teilweise keine vollständigen Datensätze vor. Daher konnten ca. 20 Fälle nicht untersucht werden.

In der Analyse mit „Vitaler Erschöpfung vorhanden/nicht vorhanden“ zeigte sich kein Hauptoder Interaktionseffekt.

In der Analyse entsprechend der 3.2.2.2.1 Unterhypothese A (S.51) mit den stetigen Werten der Skala von Vitaler Erschöpfung war lediglich der Effekt der Ln HF in der Ruhephase nicht mehr nachweisbar. Die signifikanten Effekte auf die mittlere HR in der Gehphase und Ln LF/HF in Taktatmungs- und Ruhephase waren weiterhin nachweisbar. Auch hier zeigte sich ein Zusammenhang zwischen zunehmender Vitaler Erschöpfung und niedrigerer mittlerer HR in der Gehphase und niedrigerer Ln LF/HF in Taktatmungs- und Ruhephase (s. S.51).

\subsection{Körperliche Funktionsfähigkeit (Hypothese 2)}

321 Werte für körperliche Funktionsfähigkeit lagen anhand des SF-36 unter Ausschluss der bearbeiteten EKGs vor. Von diesen lagen aufgrund von Ausschlüssen teilweise keine vollständigen Datensätze vor. Daher konnten ca. 30 Fälle nicht untersucht werden.

Für die dichotome Bewertung der körperlichen Funktionsfähigkeit zeigte sich nur ein Haupteffekt für Ln LF/HF und ein Interaktionseffekt für mittlere HR.

Für die Analyse analog der Untersuchung unter 3.2.2.3.1 Unterhypothese A (S.56) mit den stetigen Werten für körperliche Funktionsfähigkeit als Prädiktor zeigten sich im Rahmen der linearen Regression fast alle Effekte, wie unter Unterhypothese A angegeben. Lediglich die Effekte von Ln SDNN und Ln Total waren nicht mehr nachweisbar (s. ab S.56). 


\subsubsection{Nur Probanden ohne Betablocker}

Betablocker haben einen starken Effekt auf die HRV-Parameter. Daher war angenommen worden, dass die Vormedikation mit Betablocker das Ergebnis verfälschen könnte. Um den Einfluss der Betablockervormedikation auf das Ergebnis der Hypothesenprüfung zu eruieren wurde nach der in Hypothesen 1 und 2 beschriebenen Art und Weise eine explorative Analyse ohne Probanden mit Betablockern durchgeführt.

Insgesamt 235 Probanden nahmen keine Betablocker ein (s. Tabelle 5, S.38). Mindestens 211 Probanden standen aufgrund teilweise fehlender Fragebogendaten zur weiteren Analyse zur Verfügung. Von den 235 Probanden ohne Betablocker waren 24 Probanden auffällig für Depressivität, 41 Probanden auffällig für Angst, 43 Probanden auffällig für Vitale Erschöpfung und 36 Probanden für schlechte körperliche Funktionsfähigkeit.

Eine Repräsentativitätsprüfung der Kollektive bezüglich des Kriteriums Betablockermedikation vorhanden beziehungsweise nicht vorhanden wurde anhand der unter Repräsentativitätsprüfung (s. 2.5.3.1 Repräsentativitätsprüfung, S.34) aufgeführten Kriterien durchgeführt. Im Rahmen der Chi-Quadrat-Testung zeigte sich eine Abhängigkeit zwischen Vormedikation mit Betablockern und der Vorerkrankung KHK beziehungsweise MI. In der Gruppe mit Betablockervormedikation waren mehr Fälle dieser beiden Erkrankungen vorhanden, was damit zu begründen ist, dass Betablocker jeweils zum Therapieschema gehören.

\subsection{Depressivität (Hypothese 1)}

Für Depressivität zeigte sich weder in der dichotomen noch in der stetigen Analyse ein Haupteffekt für die HR oder die HRV-Parameter. Lediglich in der dichotomen Analyse waren Interaktionseffekte mit der Zeit für Ln LF, Ln HF und Ln Total erkennbar. Daraufhin wurde nach Untersuchungsphasen aufgetrennt untersucht. Hier zeigten sich keine signifikanten Effekte. Damit sind für nur Probanden ohne Betablocker keine signifikanten Effekte der Depressivität auf die HRV-Parameter nachweisbar (vergleiche Hypothese 1 ab S. 43).

\subsection{Angst (Hypothese 2)}

Für Angst stetig und dichotom zeigten sich keine Haupt-oder Interaktionseffekte. Damit keine Veränderung zu den vorangegangenen Analysen (vergleiche Hypothese 2 Angst ab S. 50). 


\subsection{Vitale Erschöpfung (Hypothese 2)}

Für Vital Erschöpfte zeigte sich in der Analyse für „Vitale Erschöpfung vorhanden/ nicht vorhanden“ im Rahmen eines Allgemeinen Linearen Modells mit Messwiederholungen kein Haupt- und kein Interaktionseffekt.

Für die Analyse mittels linearer Regression analog des unter 3.2.2.2.1 Unterhypothese A (S.51) beschriebenen Verfahrens für den stetigen Wert der Vitalen Erschöpfung als Prädiktor zeigte sich lediglich für mittlere HR ein inverser Effekt in der Gehphase. Zudem zeigte sich ein konkordanter Effekt der Vitalen Erschöpfung auf die pNN50 und ein inverser Effekt auf Ln LF/HF in der Ruhephase. Der Effekt der Ln HF und der Effekt der Ln LF/HF in der Taktatmungsphase waren nicht mehr nachweisbar. Der konkordante Effekt der VE auf pNN50 in der Ruhephase war in der primären Analyse bisher nicht zu beobachten gewesen (s. ab S.51). Erneut waren in der explorativen analog der primären bei zunehmender Vitaler Erschöpfung die Werte der mittleren HR in der Gehphase und der Ln LF/HF in der Ruhephase abnehmend (s. ab S.51).

\subsection{Körperliche Funktionsfähigkeit (Hypothese 2)}

Für die körperliche Funktionsfähigkeit zeigten sich in der dichotomen Analyse für niedrige beziehungsweise hohe körperliche Funktionsfähigkeit im Rahmen eines Allgemeinen Linearen Modells Haupteffekte für Ln LF, Ln Total und Ln LF/HF und ein Interaktionseffekt für mittlere HR.

Die Analyse analog der primären Analyse in 3.2.2.3.1 Unterhypothese A (S.56) durchgeführt, zeigte einen signifikanten Einfluss des stetigen Wertes für körperliche Funktionsfähigkeit in einer linearen Regression auf die HRV-Parameter wie unter 3.2.2.3.1 Unterhypothese A (S.56) beschrieben, bis auf die signifikanten Effekt für Ln rMSSD und Ln HF in der Gehphase. Diese Effekte für Ln rMSSD und Ln HF waren in der Analyse ohne Probanden mit Betablockermedikation nicht mehr nachweisbar (vergleiche ab S. 56).

Im Diskussionsteil erfolgt nun die kritische Wertung dieser Ergebnisse. 


\section{Diskussion}

Die HRV wurde im Rahmen dieser Studie in verschiedenen autonomen Aktivierungszuständen analysiert. Primäres Ziel war es, den Zusammenhang zwischen der erhobenen HR und HRV und den psychosomatischen Variablen „Depressivität“, „Angst“, „Vitale Erschöpfung“ und „körperlicher Funktionsfähigkeit“ zu untersuchen. Es sollte geprüft werden, ob durch die durchgeführte Analyse an einem großen Kollektiv in verschiedenen autonomen Aktivierungszuständen mit großen zum Vergleich vorliegenden Untergruppen die Uneinheitlichkeit der Befunde, wie in der Einleitung beschrieben, bezüglich der autonomen Dysfunktion bei psychischen Auffälligkeiten geklärt werden kann.

Die Ergebnisse der Hypothesenprüfung werden nach psychosomatischer Erlebensdimension aufgetrennt kritisch bewertet.

Aufgrund der somatischen Eigenheiten des untersuchten Kollektivs, inbesondere der vorhandenen kardialen Vorerkrankungen, wie in der deskriptiven Statistik dargestellt, war befürchtet worden, dass ein Effekt für die untersuchten psychosomatischen Variablen durch eine aufgrund der somatischen Vorerkrankungen vorhandene HRV-Veränderung vorgetäuscht werden könnte. Daher wurde eine Analyse des Einflusses der somatischen Vorerkrankungen auf die HRV durchgeführt. Vor der Darstellung der Ergebnisse bezüglich der psychosomatischen Variablen soll auf das Ausmaß des Einflusses der in diesem Kollektiv relevanten somatischen Faktoren auf die HR und HRV-Parameter eingegangen werden.

\subsection{Zusammenhänge von HRV-Parametern mit kardialen Vorerkran- kungen und kardialen Schweregradmarkern}

Insbesondere für die vagalen Parameter war für die im Kollektiv vorhandenen Vorerkrankungen sowie für die zunehmende Verschlechterung der kardialen Schweregradmarker eine Erniedrigung der vagalen HRV-Parameter (rMSSD, pNN50 und HF) erwartet worden. Diese Veränderungen zeigten sich für rMSSD, pNN50 und HF in einzelnen Phasen für zunehmenden BMI und zunehmenden Alkoholkonsum pro Woche (s. Tab. 14 und 15 ab S.63). Im Gegenteil zu den erwarteten Veränderungen zeigten sich für einige der kardialen Vorerkrankungen und für höhere kardiale Schweregradmarker Zeichen eines erniedrigten Sympathikotonus und erhöhten Vagotonus. Es wurde vermutet, dass dies an der Vormedikation mit Betablockern liegen könnte. 
Zum Ausschluss einer Vortäuschung von Effekten durch die Vormedikation mit Betablockern wurde eine explorative Analyse durchgeführt, deren Ergebnisse in der weiteren Diskussion bewertet werden.

Eine Erniedrigung der HRV-Parameter rMSSD, pNN50, HF insbesondere in der Gehphase für steigenden BMI, wie in der vorliegenden Studie beobachtet, könnte durchaus einem frühen Zeichen einer nervalen Schädigung entsprechen, wie auch schon in anderen Arbeiten für übergewichtige Probanden beobachtet (z.B. TENTOLOURIS et al. 2008), die in einer körperlichen Belastungssituation als erstes in Erscheinung tritt (s. Tabelle 14, S. 63).

Für rMSSD und HF zeigte sich in der Gehphase zusätzlich für zunehmenden Alkoholkonsum pro Woche ein Zusammenhang mit erniedrigten HRV-Parametern (s. Tabelle 14, S. 63). Ein Zusammenhang zwischen Alkoholkonsum und erniedrigten HRV-Parametern ist bereits beschrieben worden (RAJENDRA ACHARYA et al. 2006). Vermutet werden könnte auch hier ein sich unter körperlicher Belastung früh demaskierender Effekt des Alkoholkonsums auf die HRV-Parameter.

Wie in diesem Kapitel erläutert, zeigten sich einzelne Effekte, allerdings nicht so ausgeprägt wie befürchtet, der kardialen Vorerkrankungen auf die HR und die HRV-Parameter. In der Analyse der psychosomatischen Variablen und ihres Zusammenhanges zur HRV wurde im Rahmen der Unterhypothese B auf Einfluss dieser Variablen kontrolliert und dieser soweit relevant in den folgenden Kapiteln erwähnt.

\subsection{Depressivität}

In dieser Arbeit wird eine milde Depressivität, gemessen anhand des HADS, untersucht (HERRMANN-LINGEN et al. 2005). Männer und Frauen dieses Kollektivs waren mit ca. 13\% ähnlich oft depressiv (s. Tab. 7, S.40). Üblicherweise zeigen Frauen eine höhere Prävalenz der Depression in der deutschen Bevölkerung (ROBERT-KOCH-INSTITUT 2006). Bei kardiologischen Patienten sind 15-20\% depressiv (HERRMANN 1997). Das untersuchte Kollektiv erscheint daher im Vergleich mit anderen kardiologisch erkrankten Kollektiven etwas weniger depressiv, eine Geschlechtsdominanz fehlt.

Für Depressivität war im Rahmen der Analyse der HRV eine vermehrte sympathische und eine erniedrigte vagale Innervation im Sinne einer autonomen Dysfunktion erwartet worden. In der statistischen Prüfung zeigte sich für keinen der untersuchten HRV-Parameter dieser vermutete Zusammenhang. 
Die beobachteten nicht hypothesenkonformen Veränderungen sollen nun nach Untersuchungsphase getrennt analysiert werden.

In der Gehphase zeigte sich für die mittlere HR ein signifikant niedrigerer Mittelwert für die Depressiven (s. Tab. 10, S.44). Dieser Zusammenhang scheint durch eine mangelnde Motivation und daraus resultierende geringere körperliche Belastung im 6-Minuten-Gehtest bedingt zu sein. Diese Annahme wird auch durch den klaren Zusammenhang von kürzerer Gehstrecke und steigenden Depressivitätswerten unterstützt (s. Tab. 16, S.66). In der Testung auf Abhängigkeit von kardialen Schweregradmarkern war die Gehteststrecke klar konkordant mit der mittleren HR korreliert (s. Tab. 14, S.63). Auch hierin zeigt sich der Zusammenhang von stärkerer körperlicher Aktivität mit begleitender höherer Sympathikusaktivierung erkennbar an der steigenden mittleren HR und dabei längerer Gehteststrecke.

Dieser Effekt der Depressivität in der Gehphase war in der primären Analyse auf somatische Mediatoren oder Confounder zumindest teilweise unabhängig von den untersuchten somatischen Variablen (s. 3.2.1.2 Unterhypothese B, S.46). In der geschlechtergetrennten Betrachtung war er nicht mehr nachweisbar (s. 3.2.1.4 Unterhypothese D, S.49). Bei der geschlechtergetrennten Betrachtung ist natürlich auch an einen Verlust der Power in den Subgruppen zu denken.

In der explorativen Analyse mit nur unbearbeiteten EKGs (s. 3.2.6.1.1, S.68) zeigte sich für die Gehphase ein zusätzlich signifikant gewordener Mittelwertsunterschied für LF und TotalHRV. Diese HRV-Parameter waren für Depressive erhöht. Bei längerer Gehteststrecke und anzunehmender erhöhter Sympathikusaktivität und erniedrigter Vagusinnervation zeigten sich abnehmende LF- und Total-HRV-Werte (s. Tab. 14, S.63). Auch diese Veränderungen scheinen zu dem vermuteten Zusammenhang zwischen Depressivität und mangelnder Motivation zu passen, beziehungsweise zu vergleichsweise geringerer körperlicher Aktivität der Depressiven im Gehtest.

In Taktatmungs- und Ruhephase zeigte sich jeweils nur ein signifikanter Zusammenhang zwischen Depressivität und HRV nämlich für LF/HF. Der Mittelwert der LF/HF war im Gruppenvergleich für Depressive jeweils erniedrigt (s. Tab. 10, S.44).

Für Taktatmungs- und Ruhephase entsprechen die niedrigeren Werte für die Depressiven für LF/HF vermutlich wenigstens zum Teil einer verminderten Sympathikusstimulation (TASK FORCE OF THE EUROPEAN SOCIETY OF CARDIOLOGY AND THE NORTH AMERICAN SOCIETY OF PACING AND ELECTROPHYSIOLOGY 1996). 
In der Taktatmungsphase könnten die niedrigen Werte für LF/HF bei den Depressiven ein Zeichen erniedrigter Resonanzfähigkeit oder aber auch mangelnder Kooperation bei der Taktatmung sein. Durch die Taktatmung wäre ein Anstieg des LF und damit auch des LF/HF Quotienten erwartet worden (s. 1.3.3.2 Atmung, S.10).

In der Ruhephase bleibt unklar, warum hier für Depressive ein niedrigerer Wert für LF/HF nachzuweisen war.

Diese Effekte der Depressivität in der Taktatmungs- und Ruhephase waren in der primären Analyse auf somatische Mediatoren oder Confounder zumindest teilweise unabhängig von den somatischen Variablen (s. 3.2.1.2 Unterhypothese B, S.46), aber in der geschlechtergetrennten Betrachtung nur für Frauen nachvollziehbar (s 3.2.1.4 Unterhypothese D, S.49). Bei der geschlechtergetrennten Betrachtung ist erneut an einen Verlust der Power in den Subgruppen zu denken.

In der explorativen Analyse für nur unbearbeitete EKGs war der Effekt der Depressivität auf die LF/HF in Taktatmungs- und Ruhephase weiter nachweisbar (s. 3.2.6.1.1, S.68).

Für Depressivität war in der explorativen Analyse für lediglich Probanden ohne die Vormedikation mit Betablocker kein Effekt auf die HR und die HRV-Parameter nachvollziehbar (s. 3.2.6.2.1, S.68). Auch schon im Rahmen der Unterhypothese B hatte sich ein neben der Depressivität signifikanter unabhängiger Effekt der Vormedikation Betablocker auf mittlere HR und LF/HF nachweisen lassen (s. 3.2.1.2 Unterhypothese B, S.70). Das Fehlen jeglicher, in der Hauptanalyse gefundener Effekte in dieser eplorativen Analyse kann dem Einfluss der Betablocker geschuldet sein. Möglich ist aber auch ein Verlust der Power in den Subgruppen bei Reduktion des insgesamt betrachteten Kollektivs auf knapp die Hälfte.

Wie bereits in der Einleitung unter 1.3.5 Psychische Vorerkrankungen (S.12) erwähnt, sind die in der Literatur beschriebenen Zusammenhänge von Depressivität und HRV nicht einheitlich (ROTTENBERG 2007).

Insgesamt kann in dieser großen Analyse von HRV an N=468 Probanden mit kardiovaskulären Risikofaktoren und zum Teil kardialen Erkrankungen für die hier untersuchte überwiegend leichte Form der Depressivität keine autonome Dysfunktion im Sinne einer reduzierten vagalen Kontrolle und erhöhten sympathischen Innervation nachgewiesen werden. 
Dies widerspricht der Annahme, dass Depressivität mit erniedrigter vagaler und erhöhter sympathischer Innervation einhergeht. Diese Annahme entstammt verschiedenen Studien, die bei körperlich gesunden Depressiven erhöhte Katecholaminwerte(CARNEY et al. 2002) oder erhöhte Blutdruckwerte nachgewiesen haben (DAVYDOV et al. 2007). Im Tierexperiment konnten bei simulierter Depressivität erhöhte basale HR-Werte und eine signifikant erniedrigte HRV (SDNN) nachgewiesen werden (GRIPPO et al. 2002). In einer relativ kleinen Studie $(\mathrm{N}=10)$ von NAHSHONI et al. an Patienten unter SSRI-Therapie mit Major Depression ohne Kontrollgruppe wurde ein Zusammenhang der Major Depression mit stark erniedrigten HRVWerten gefunden. Das verwendete Studiendesign sah eine Messung der HRV in Rückenlage über 10 Minuten vor (NAHSHONI et al. 2004). Aufgrund der fehlenden Kontrollgruppe, der geringen Gruppengröße und der fehlenden Kontrolle für die SSRI-Vormedikation ist das erzielte Ergebnis als kritisch zu bewerten.

In der vorliegenden Studie erscheinen die Effekte insbesondere der Geh- und Taktatmungsphase am ehesten einem Mangel an Motivation und korrekter Mitarbeit geschuldet zu sein, wobei erneut zu betonen ist, dass in der Analyse ohne Probanden mit Betablocker die beobachteten Effekte nicht mehr nachzuweisen waren. Eine stärkere Interaktion dieser Vormedikation Betablocker mit Depressivit ist nicht auszuschließen. Für Betablocker ist im Nebenwirkungsspektrum ein depressiogener Effekt bisher postuliert worden. Aktuelle Studien belegen dies allerdings teilweise nicht (HOOGWEGT et al. 2012) oder sogar das Gegenteil (BATTES et al. 2011).

Die erbrachten Ergebnisse unterstützen aufgrund der wohl hauptsächlich durch Motivationsmangel und mangelnde Mitarbeit in der Taktatmungsphase entstandenen Effekte und fehlende weitere Effekte eher die Resultate von SAYAR et al. (2002). In einer Fall-Kontroll-Studie (21 Probanden pro Gruppe) mit körperlich gesunden Patienten, die an einer Major Depression litten (Durchschnittsalter 38 Jahre), konnten SAYAR et al. 2002 zeigen, dass sich kein Zusammenhang mit den über 24 Stunden erhobenen HRV-Parametern für Major Depression zeigte. Auch bei einem teilweise somatisch erkrankten Kollektiv, wie dem hier vorliegenden, gibt es Arbeiten die keinen Zusammenhang zwischen Depression und HRV zeigen, wie die Arbeit von EINVIK et al. im Jahre 2011, in der für 290 Probanden -im Mittel 48 Jahre alt kein signifikanter Effekt von Major Depression auf die HRV nachgewiesen werden konnte. Auch in jener Arbeit waren die HRV-Parameter, wie bei SAYAR et al. (2002), über mehrere Stunden erhoben worden (EINVIK et al. 2011). 
Zusammenfassend kann spekuliert werden, dass bei der Arbeit von NAHSHONI et al. 2004, die HRV bei körperlich gesunden Depressiven untersucht haben, möglicherweise eine Medikamentenwirkung als Effekt der Depressivität gewertet wurde und, wie bereits SAYAR et al. 2002 spekulierte, in früheren Arbeiten bei körperlich kranken Depressiven ein Effekt von somatischer Erkrankung einen Effekt für Depressivität vorgetäuscht haben könnte.

Möglich ist allerdings auch, dass ein Effekt leichterer Depressivität, gemessen anhand der HADS, wie in dieser Arbeit verwendet, im Vergleich zu Patienten mit einer Major Depression keinen starken Effekt auf die HRV hat, also ein eventuell vorhandener Effekt für starke Depressivität aufgrund der mutmaßlich geringen Prävalenz schwerer Depression im Untersuchungskollektiv nicht gefunden werden konnte. Dazu ist allerdings anzumerken, dass auch die Arbeiten von SAYAR et al. und EINVIK et al. selbst für Patienten mit Major Depression keinen Effekt auf die HRV fanden (EINVIK et al. 2011, SAYAR et al. 2002).

\subsection{Angst}

Das untersuchte Konstrukt bezüglich Angst im HADS ist das einer generalisierten, frei flottierenden Angstsymptomatik (HERRMANN-LINGEN et al. 2005). Im Kollektiv sind signifikant mehr Frauen (19 \%) auffällig ängstlich als Männer (14\%) (s. Tabelle 7, S.40). Diese Dominanz der Frauen entspricht in etwa der Verteilung in der deutschen Gesamtbevölkerung (ROBERT-KOCH-INSTITUT 2006). Bei kardiologischen Patienten sind auffällige Angstwerte gemessen mittels des HADS bei $19 \%$ beschrieben. Auch hier zeigen Frauen höhere Werte (HERRMANN 1997). Das vorliegende Kollektiv präsentiert sich im Vergleich zu kardiologischen Kollektiven ähnlich ängstlich mit einer leichten Geschlechtsdominanz der Frauen.

Für Angst war eine vermehrte sympathische und eine erniedrigte vagale Innervation im Sinne einer autonomen Dysfunktion erwartet worden. In der statistischen Prüfung zeigte sich weder in der primären, noch in der explorativen Analyse eine Veränderung in diese Richtung.

Insgesamt kann in dieser großen Analyse von HRV an N=468 Probanden mit kardiovaskulären Risikofaktoren und zum Teil kardialen Erkrankungen für die hier untersuchte Form der Angst keine autonome Dysfunktion im Sinne einer reduzierten vagalen Kontrolle und erhöhten sympathischen Innervation nachgewiesen werden. 
Dies widerspricht Arbeiten zu Angststörungen, die erhöhte HR und erniedrigte HRV-Werte gemessen hatten (FRIEDMAN 2007; ROTTENERG 2007). Auch im Tiermodell ist eine erniedrigte HRV mit starker Angst verbunden gewesen (GABURRO et al. 2011; FRIEDMAN 2007). In der Regel wurden erprobte Stresstests innerhalb der Studien durchgeführt, die eine angstbesetzte Stresssituation imitieren sollen (FRIEDMAN 2007). In der vorliegenden Studie wurden keine solchen Methoden angewandt.

Vereinzelt gibt es allerdings auch Arbeiten, die keinen Zusammenhang zwischen HRV und Angst sehen (EINVIK et al. 2011; FRIEDMAN 2007). Bei EINVIK et al. 2011 war ebenfalls kein Stresstest angewandt worden. Diese Arbeiten können mit den Ergebnissen der vorliegenden Studie unterstützt werden.

Auch die kardialen Befunde zeigten keinen Zusammenhang mit Angst (s. Tab. 16, S.66). Auch hier sind die in der Literatur (s. 1.4 Kardiale Schweregradmarker und ihr Zusammenhang mit HRV und Psyche, S.13) beschriebenen Veränderungen in dem untersuchten Kollektiv nicht nachzuvollziehen.

Zusammenfassend ist am ehesten davon auszugehen, dass in der vorliegenden Studie aufgrund des Fehlens einer akuten Stresssituation im experimentellen Design keine signifikanten Zusammenhänge zwischen Angst und HRV nachzuweisen waren. Zusätzlich lag bei den meisten Patienten lediglich eine leichte Symptomatik der Angst vor, auch diese Tatsache könnte einen eventuell vorhandenen Effekt starker Angst verborgen haben. Schließlich ist nach den analysierten Ergebnissen zu vermuten, dass Angstsymptomatik eher passager in akuten Angst auslösenden Situationen zu einer Veränderung der HRV und eine milde Ängstlichkeit nicht generell zu einer Veränderung des Aktivitätsniveaus des ANS führt.

\subsection{Vitale Erschöpfung}

Das Konstrukt der Vitalen Erschöpfung beschreibt ein Gefühl der Erschöpfung, und Demoralisation (LADWIG et al. 2008). Im untersuchten Kollektiv sind signifikant mehr Frauen (23\%) vital erschöpft als Männer (15\%) (s. Tabelle 7, S.40). Dies entspricht den auch in anderen Arbeiten beobachteten häufiger erhöhten Werten für Frauen (WILLIAMS et al. 2010). Die Prävalenz der Vitalen Erschöpfung bei kardiologischen Patienten wird teilweise um 3560\% beschrieben (KOP 1999). In dieser Arbeit sind es lediglich 15\% der Männer und 23\% der Frauen. Die im untersuchten Kollektiv vorliegenden Werte sind, wie erwartet, höher bei den Frauen, aber insgesamt weniger hoch, als bei einem kardial vorerkrankten Kollektiv zu erwarten wäre. Am ehesten ist dies dadurch zu begründen, dass im untersuchten Kollektiv 
neben einigen bereits kardial erkrankten Probanden überwiegend Probanden mit lediglich kardialen Risikofaktoren eingeschlossen wurden, die oft subjektiv asymptomatisch waren.

Für Vitale Erschöpfung war im Rahmen der Analyse der HRV eine vermehrte sympathische und eine erniedrigte vagale Innervation im Sinne einer autonomen Dysfunktion erwartet worden. In der statistischen Prüfung zeigte sich für keinen der untersuchten HRV-Parameter diese vermutete Veränderung.

Die beobachteten nicht hypothesenkonformen Veränderungen sollen nun nach Untersuchungsphasen getrennt analysiert werden. Bis auf für den Parameter HF zeigten sich für Vitale Erschöpfung in denselben Phasen Zusammenhänge mit denselben Parametern der HRV wie für Depressivität im Kapitel 4.2 Depressivität (S. 73) bereits diskutiert wurde.

In der Gehphase zeigte sich für mittlere HR ein signifikanter inverser Zusammenhang. Bei zunehmendem Ausmaß der Vitalen Erschöpfung war die mittlere HR in der Gehphase niedriger (s. Tabelle 12, S.51). Hier scheint es sich analog zu den Veränderungen, die auch schon für die Depressiven für mittlere HR in der Gehphase beobachtet wurden, um eine geringere Motivation im Rahmen der Gehphase und damit geringere körperliche Aktivität entsprechend einer geringeren Aktivierung des Sympathikus bei den Vital Erschöpften zu handeln. Diese Annahme wird durch den klaren Zusammenhang von verkürzter Gehstrecke bei steigendem Ausmaß der Vitalen Erschöpfung unterstützt (s. Tab. 16, S.66). In der Testung auf Abhängigkeit von kardialen Schweregradmarkern (s. Tab. 14, S.63) war die Gehteststrecke klar konkordant mit der mittleren HR korreliert. Auch hierin zeigt sich der Zusammenhang von stärkerer körperlicher Aktivität mit begleitender höherer Sympathikusaktivierung erkennbar an der bei längerer Gehteststrecke steigenden mittleren HR.

Dieser Effekt der Vitalen Erschöpfung in der Gehphase war in der primären Analyse auf somatische Mediatoren oder Confounder zumindest teilweise unabhängig von den untersuchten somatischen Variablen (s. 3.2.2.2.2 Unterhypothese B, S.52), allerdings war in der geschlechtergetrennten Betrachtung der Effekt der VE auf die mittlere HR in der Gehphase nur für Frauen nachweisbar (s. 3.2.2.2.4 Unterhypothese D, S.55). Bei der geschlechtergetrennten Betrachtung ist neben einem Interaktionseffekt des Geschlechts auch an einen Verlust der Power in den Subgruppen zu denken.

In der explorativen Analyse der Gehphase mit nur unbearbeiteten EKGs und mit nur Probanden ohne Betablocker war der signifikante Einfluss der VE auf die mittlere HR weiter nachweisbar (s. 3.2.6.1.3, S.69, und 3.2.6.2.3, S.71). 
In der Taktatmungsphase zeigte sich ein signifikanter Zusammenhang zwischen zunehmendem Ausmaß der Vitalen Erschöpfung und sinkenden Werten für LF/HF (s. Tab. 12, S.51). Analog des auch für Depressivität in der Taktatmungsphase beobachteten Effekts mit erniedrigten Werten für Depressive könnte dies vermutlich wenigstens zum Teil einer verminderten Sympathikusstimulation entsprechen (TASK FORCE OF THE EUROPEAN SOCIETY OF CARDIOLOGY AND THE NORTH AMERICAN SOCIETY OF PACING AND ELECTROPHYSIOLOGY 1996). Wahrscheinlicher ist allerdings, dass die niedrigen Werte für LF/HF bei zunehmendem Ausmaß der Vitalen Erschöpfung, wie bei vorhandener Depressivität ein Zeichen erniedrigter Resonanzfähigkeit oder aber auch mangelnder Kooperation bei der Taktatmung sind. Durch die Taktatmung wäre ansonsten ein Anstieg des LF und damit auch des LF/HF Quotienten erwartet worden (s. 1.3.3.2 Atmung, S.10).

Für die erniedrigte LF/HF vital Erschöpfter in der Taktatmungsphase war kein eindeutiger Mediator oder Confounder identifiziert worden. Dieser Effekt der Vitalen Erschöpfung scheint zumindest teilweise unabhängig von den untersuchten somatischen Variabeln zu sein (s. 3.2.2.2.2 Unterhypothese B, S.51), allerdings war in der geschlechtergetrennten Betrachtung der Effekt der VE auf die LF/HF in der Taktatmungsphase nur für Frauen nachweisbar (s. 3.2.2.2.4 Unterhypothese D, S.54). Erneut ist hier sowohl eine Interaktion des Geschlechts als auch ein Effektverlust aufgrund der kleineren Gruppen möglich.

In der explorativen Analyse der Taktatmungsphase mit nur unbearbeiteten EKGs war der Effekt der Erschöpfung auf die LF/HF in der Taktatmungsphase weiter nachweisbar. In der explorativen Analyse mit nur Probanden ohne Betablocker war der signifikante Einfluss der VE auf die LF/HF dagegen nicht mehr nachweisbar (s. 3.2.6.1.3, S.69, und 3.2.6.2.3, S.71).

In der Ruhephase zeigte sich ein signifikanter Effekt der Vitalen Erschöpfung auf HF und LF/HF. Ein zunehmendes Ausmaß der Vitalen Erschöpfung war mit steigenden Werten der HF und sinkenden Werten der LF/HF verbunden. Auch hier zeigt sich für LF/HF eine Analogie zu den Ergebnissen zur Depressivität. Für diese waren ebenfalls die Werte von LF/HF in der Ruhephase erniedrigt. Aufgrund der steigenden HF-Werte, die einem erhöhten Vagotonus entsprechen, sinkt der Wert des Quotienten LF/HF. Hier kann der sinkende Wert der LF/HF allenfalls zum Teil einer verminderten Sympathikusstimulation entsprechen (TASK FORCE OF THE EUROPEAN SOCIETY OF CARDIOLOGY AND THE NORTH AMERICAN SOCIETY OF PACING AND ELECTROPHYSIOLOGY 1996). 
Diese Effekte der Vitalen Erschöpfung in der Ruhephase waren in der primären Analyse auf somatische Mediatoren oder Confounder zumindest teilweise unabhängig von den somatischen Variablen (s. 3.2.2.2.2 Unterhypothese B, S.51), aber in der geschlechtergetrennten Betrachtung war der Effekt für HF nicht mehr, der Effekt für LF/HF nur für Frauen nachvollziehbar (s 3.2.2.2.4 Unterhypothese D, S.54).

In der explorativen Analyse der Ruhephase war für unbearbeitete EKGs der Effekt für Erschöpfung auf LF/HF weiter nachweisbar, derjenige auf HF nicht mehr. Vermutlich war hier der Effekt zu klein und aufgrund des Verlusts der Power in den Subgruppen nicht mehr nachweisbar. In der explorativen Analyse für lediglich Probanden ohne die Vormedikation Betablocker war für Vitale Erschöpfung neben dem bekannten inversen Effekt auf LF/HF ein konkordanter Effekt auf pNN50 zu verzeichnen. Der Effekt der VE auf HF war auch in der Analyse mit nur Probanden ohne Betablocker nicht mehr nachweisbar (s. 3.2.6.2.3 Vitale Erschöpfung (Hypothese 2), S.71). Der Effekt für pNN50 ist möglicherweise ein Artefakt der multiplen Testung.

Die Effekte der VE auf die mittlere HR in der Gehphase und die LF/HF in der Ruhephase, die auch in der Analyse ohne Probanden mit Betablockervormedikation nachweisbar waren, scheinen stabile Effekte zu sein. Für den Einfluss der VE auf mittlere HR in der Gehphase könnte spekuliert werden, dass die geringere Aktivität in der Gehphase auch durch körperliche Schwäche aufgrund kardialer Erkrankung bedingt ist. Dies kann auch durch den Zusammenhang von steigender VE und steigendem NT-pro-BNP unterstützt werden (s. Tab. 16, S.66). Zum Beispiel für männliche Herzinsuffzienzpatienten ist NT-pro-BNP als unabhängiger Prädiktor der Gehteststrecke analysiert worden. Steigende NT-pro-BNP-Werte korrelierten mit abnehmender Gehstrecke (WEGRZYNOWSKA-TEODORCZYK et al. 2010).

Alternativ ist für die Zusammenhänge zwischen VE und mittleren HR in der Gehpase und LF/HF in der Ruhephase auch die hormonelle Wirkung der bei zunehmender VE steigenden NP (s. erneut Tab. 16, S.66) mit Vagusstimulation und Bradykardie (HERRING et al. 2001) als Erklärung möglich. Dies könnte als Zeichen einer tatsächlich schwereren kardialen Dysfunktion der Erschöpften gewertet werden. 
Insgesamt kann in dieser großen Analyse von HRV an $\mathrm{N}=468$ Probanden mit kardiovaskulären Risikofaktoren und zum Teil kardialen Erkrankungen für Vitale Erschöpfung keine autonome Dysfunktion im Sinne einer reduzierten vagalen Kontrolle und erhöhten sympathischen Innervation nachgewiesen werden.

Bezüglich der Vitalen Erschöpfung wurden kaum Arbeiten zu HRV in der Pubmed-Recherche (s. 1.3.5 Psychische Vorerkrankungen, S.12) gefunden. Lediglich WATANABE et al. haben 2002 HRV und VE erforscht und eine erniedrigte HF für Vital Erschöpfte gefunden (WATANABE et al. 2002).

Dieses Ergebnis von WATANABE et al. (2002) lässt sich durch die vorliegenden Befunde nicht bestätigen. Ganz im Gegenteil wurde in der vorliegenden Studie eine erhöhte HF in der Ruhephase bei zunehmender Vitaler Erschöpfung gefunden (s. Tab. 12, S.51), allerdings war dieser Effekt nicht mehr in der geschlechtsgetrennten Betrachtung (s. 3.2.2.2.4 Unterhypothese D, S.54) oder in den explorativen Analysen nachweisbar (s. S.69 und S.71). Hier scheint ein sehr kleiner Effekt beziehungsweise ein durch Interaktionen vorgetäuschter Effekt vorzuliegen.

WATANABE et al. haben eine Kurzzeitmessung von 5 Minuten der HF in Rückenlage bei einer Atemfrequenz von $0,25 \mathrm{~Hz}$ (entspricht 15 Atemzügen pro Minute) an 52 Probanden (Alter zwischen 30 und 55 Jahren) durchgeführt. Die VE wurde in drei Gruppen nach Höhe des Scores eingeteilt: „hohe VE“ $(\mathrm{N}=10)$, „moderate VE“ $(\mathrm{N}=2)$ und „niedrige VE“ $(\mathrm{N}=20)$. Hieraus ist eine Kollektivgröße von lediglich N= 32 nachzuvollziehen. Möglicherweise liegt ein Tippfehler vor und die Gruppe mit moderater VE hatte 22 Probanden. Der signifikante Effekt der VE auf HF wurde im Vergleich der Gruppe „hohe VE“ und „moderate VE“ gefunden (WATANABE et al. 2002).

Im Gegensatz dazu wurde die vorliegende Untersuchung an deutlich mehr Probanden, damit statistisch aussagekräftiger, durchgeführt. Die untersuchten Probanden waren alle über 50 Jahre alt. Höheres Alter ist mit niedrigen HRV-Werten in Verbindung gebracht worden (RAJENDRA ACHARYA et al. 2006). Die EKG-Analyse fand im Sitzen bei frei wählbarer Atemfrequenz statt. Die normale Atemfrequenz für Erwachsene liegt um 15 pro Minute. Damit ist die starke Atemabhängigkeit der HF (TASK FORCE OF THE EUROPEAN SOCIETY OF CARDIOLOGY AND THE NORTH AMERICAN SOCIETY OF PACING AND ELECTROPHYSIOLOGY 1996) nicht sehr relevant für den Vergleich der beiden Studiener- 
gebnisse. Die sitzende Position würde eher eine geringere Vagusinnervation (niedrige HF) aufgrund der vermehrten Sympathikusaktivität zur Aufrechterhaltung des Herzzeitvolumens aufgrund des Volumenverlust in den venösen Gefäßen der Beine erwarten lassen. Eine liegende Position sollte also eher höhere vagale Innervation (höhere HF) erwarten lassen.

Möglicherweise handelt es sich bei dem für HF von WATANABE et al. 2002 beobachteten Effekt um die Auswirkungen eines aktuell unbekannten Confounders.

Zusammenfassend kann spekuliert werden, dass bei der Arbeit von WATANABE et al. 2002 ein möglicherweise, aufgrund von Besonderheiten des Kollektivs vorhandener, signifikanter Zusammenhang für steigende VE zu steigender HF beschrieben wurde. Ein solcher Effekt konnte in der vorliegenden Studie nicht bestätigt werden. Die in der vorliegenden Studie beobachteten Zusammenhänge zwischen zunehmender VE und erniedrigter mittlerer HR bei körperlicher Belastung und erniedrigter LF/HF im Sitzen können möglicherweise einem tatsächlich vorhandenen Effekt kardialer Dysfunktion bei mit zunehmendem Ausmaß der VE steigendem NT-pro-BNP und dem parasympathikomimetischen Effekts der NPs entsprechen.

\subsection{Körperliche Funktionsfähigkeit}

Im untersuchten Kollektiv waren signifikant mehr Frauen (21\%) als Männer (14\%) in der Gruppe der niedrigen körperlichen Funktionsfähigkeit (definiert als Werte kleiner 60). Für Frauen lagen die Werte des SF-36 für körperliche Funktionsfähigkeit im Mittel bei 74, bei Männern bei 79 von 100 maximal möglichen Punkten (s. Tab. 7, S.40).

Für gesunde Patienten zeigten sich in der deutschen Normstichprobe insgesamt im Mittel Werte für körperliche Funktionsfähigkeit von 96. Bei kardialen Vorerkrankungen, wie in der vorliegenden Stichprobe vorhanden, zeigten sich im Mittel Werte von 60-70 für körperliche Funktionsfähigkeit (BULLINGER und KIRCHBERGER 1998). Die körperliche Funktionsfähigkeit, als Parameter der subjektiven Lebensqualität, ist in dem vorliegenden Kollektiv im Mittel etwas besser als für kardial Erkrankte, da im untersuchten Kollektiv viele Probanden lediglich aufgrund des Vorliegens kardialer Risikofaktoren eingeschlossen worden waren. Wie in der Normstichprobe hatten Frauen niedrigere Werte für körperliche Funktionsfähigkeit (BULLINGER und KIRCHBERGER 1998). 
Für die psychosomatische Variable (selbst berichtete) körperliche Funktionsfähigkeit war zu erwarten, dass bei abnehmender körperlicher Funktionsfähigkeit auch der tatsächliche somatische Zustand der Probanden sich verschlechtern würde. Dies zeigte sich anhand einer bei abnehmender körperlicher Funktionsfähigkeit signifikant abnehmenden Gehteststrecke, einer sinkenden LVEF, einem ansteigenden Wert des diastolischen Herzinsuffizienzscores und zunehmenden Zeichen einer kardialen Volumenbelastung aufgrund des erhöhten NT-pro-BNP (s. Tab. 16, S.66).

Für niedrige körperliche Funktionsfähigkeit war im Rahmen der Hypothesenprüfung bezüglich der HR und der HRV-Parameter eine Sympathikusdominanz und ein erniedrigter Parasympathikotonus erwartet worden. In der statistischen Prüfung zeigten sich hypothesenkonforme und nicht hypothesenkonforme Zusammenhänge zwischen körperlicher Funktionsfähigkeit und der HR beziehungsweise den HRV-Parametern.

Die beobachteten Zusammenhänge sollen nun nach Untersuchungsphase getrennt diskutiert werden, wobei ebenfalls danach unterschieden wird, ob es sich um hypothesenkonforme oder nicht hypothesenkonforme Zusammenhänge handelt, die letztgenannten werden zuerst diskutiert.

In der Gehphase zeigten sich lediglich nicht hypothesenkonforme Effekte für körperliche Funktionsfähigkeit (s. Tab. 13, S.57), genauer ein signifikanter konkordanter Effekt für mittlere HR und LF/HF und ein signifikanter inverser Effekt für rMSSD und Ln HF. Bei niedrigen Werten für körperliche Funktionsfähigkeit zeigte sich ein Zusammenhang zu niedrigen Werten für die mittlere HR und LF/HF sowie hohen Werten für rMSSD und HF. Für die Taktatmungs- und Ruhephase zeigte sich ebenso ein Zusammenhang von niedriger körperlicher Funktionsfähigkeit mit niedriger LF/HF.

Diese beschriebenen Zusammenhänge entsprechen nicht dem hypothetisierten Zusammenhang zwischen HR und HRV und als niedrig empfundener körperlicher Lebensqualität. Die in den Hypothesen vermutete Sympathikusdominanz bei psychisch belasteten Probanden würde für LF/HF vermutlich eher einer Erhöhung als Zeichen des erhöhten Sympathikotonus entsprechen (TASK FORCE OF THE EUROPEAN SOCIETY OF CARDIOLOGY AND THE NORTH AMERICAN SOCIETY OF PACING AND ELECTROPHYSIOLOGY 1996). Die in der Gehphase beobachteten Zusammenhänge scheinen einer fehlenden Aktivierung des 
Sympathikus bei körperlicher Aktivität im Rahmen des Gehtests zu entsprechen (s. 6.3 HRVParameter, S.110).

Dieser vermutete Zusammenhang geringerer körperlicher Aktivität mit den beschriebenen HRV-Veränderungen für die Gehphase wird untersützt durch die Ergebnisse der Untersuchung auf Zusammenhang der Länge des Gehtests mit den verschiedenen HRV-Parametern. Hier zeigte sich jeweils für längere Gehstrecken ein Zusammenhang mit steigender mittlerer HR, steigendem LF/HF und sinkenden Werten für rMSSD und HF in der Gehphase als Zeichen einer hohen Sympathikus-und niedrigen Parasympathikusaktivität bei langen Gehteststrecken (s. Tab. 14, S.63). Auch zeigte sich für abnehmende körperliche Funktionsfähigkeit ein klarer Zusammenhang mit kürzeren Gehteststrecken (s. Tab. 16, S.66). Diese geringere Leistung im Gehtest ist aber wohl - anzunehmen aufgrund der somatisch ausgerichteten psychosomatischen Variable „körperliche Funktionsfähigkeit“- ein Resultat von körperlicher Einschränkung und weniger, wie für Depressivität und VE in Bezug auf ihren Einfluss auf mittlere HR in der Gehphase in den vorangegangenen Kapiteln diskutiert, ein Motivationseffekt, wobei dieser aber auch nicht auszuschließen ist. Dies unterstützen die vielen, auch für LF/HF in Taktatmungs- und Ruhephase beobachteten Zusammenhänge von vorhandener Vorerkrankung sowie verschlechterten kardialen Schweregradmarken in der Richtung wie für körperliche Funktionsfähigkeit beobachtet wurde (s. Tab. 14 und 15, S.63ff).

Zudem könnte der signifikante inverse Zusammenhang zwischen körperlicher Funktionsfähigkeit und NT-pro-BNP (s. Tab. 16, S.66) ein Hinweis darauf sein, dass die erhöhten Plasmaspiegel des NP zum einen eine starke kardiale Dysfunktion anzeigen und zum anderen eine Aktivierung des Sympathikus durch Verstärkung der vagalen Innervationsimpulse (HERRING et al. 2001) verhindern. Dies resultiert in einer geringeren Leistungsfähigkeit der Probanden. In einer Studie von 2010 ist für männliche Patienten mit Herzinsuffizienz NT-proBNP als unabhängiger Prädiktor der Gehteststrecke analysiert worden. Steigende NT-proBNP-Werte korrelierten mit abnehmender Gehstrecke (WEGRZYNOWSKATEODORCZYK et al. 2010).

In der explorativen Analyse mit nur unbearbeiteten EKGs waren die Effekte der körperlichen Funktionsfähigkeit in der Gehphase wie in der primären Analyse nachweisbar (s.3.2.6.1.4, S.69). In der explorativen Analyse mit nur Probanden ohne Betablockervormedikation waren in der Gehphase die Effekte für rMSSD und HF nicht mehr nachweisbar, wobei dies am ehesten durch den Verlust der Power in den Subgruppen bei Reduktion des Kollektivs auf etwa die Hälfte bedingt ist (s. 3.2.6.2.4, S.71). Für LF/HF war der Effekt der körperlichen Funktionsfä- 
higkeit in allen drei Phasen in den explorativen Analysen weiter nachweisbar (s. S. 69 und $71)$.

Nun wird auf die hypothesenkonformen Ergebnisse bezüglich des Einflusses der körperlichen Funktionsfähigkeit auf die HR- und HRV-Parameter eingegangen.

In der Gehphase zeigte sich kein hypothesenkonformer Effekt.

Lediglich in der Taktatmungsphase zeigte sich ein signifikanter hypothesenkonformer $\mathrm{Zu}$ sammenhang zwischen dem Ausmaß der körperlichen Funktionsfähigkeit und dem globalen HRV-Parameter SDNN (s. Tab. 13, S.57).

Für SDNN zeigte sich eine hypothesenkonforme Einschränkung bei zunehmender Verschlechterung der körperlichen Funktionsfähigkeit in der Taktatmungsphase. Für SDNN war für die untersuchten somatischen Variablen in der Taktatmungsphase lediglich ein signifikanter, jeweils inverser Zusammenhang mit BMI und DM nachgewiesen worden (s. Tab. 14 und 15, S.63ff). Für alle weiteren somatischen Faktoren zeigte sich kein signifikanter Zusammenhang mit SDNN. Ein steigender BMI und das Vorhandensein der Vorerkrankung DM zeigten einen klaren Zusammenhang mit niedrigeren Werten für SDNN in der Taktatmungsphase. Für das metabolische Syndrom, welches unter anderem mit einem deutlich erhöhten Gewicht einhergeht, und für DM ist bekannt, dass erniedrigte HRV-Werte vorliegen können (TENTOLOURIS et al. 2008; RAJENDRA ACHARYA et al. 2006). Außer für diese beiden Parameter scheint in dem untersuchten Kollektiv kein starker Einfluss der somatischen Variablen auf die SDNN zu bestehen. Dies bestätigte sich auch in der direkten Testung der Ergebnisse im Rahmen der Unterhypothese B auf somatische Confounder. Hier zeigte sich für SDNN in der Taktatmungsphase lediglich ein Confundereffekt für den BMI. Ansonsten war zumindest teilweise der Effekt der körperlichen Funktionsfähigkeit unabhängig von somatischen Einflussparametern (s. 3.2.2.3.2.1 Ln SDNN, S.59). Allerdings bleibt zu bemerken, obwohl in Hypothese 3 kein klarer Einfluss des Geschlechts auf die SDNN gezeigt wurde, dass der Effekt der körperlichen Funktionsfähigkeit in der geschlechtsgetrennten Untersuchung nur für Männer nachvollziehbar war (s. 3.2.2.3.4 Unterhypothese D, S.61). Hier ist wiederum ein Interaktionseffekt des Geschlechts, aber auch einfach ein Signifikanzverlust bei Gruppengrößenreduzierung möglich.

In der explorativen Analyse für nur unbearbeitete EKGs war der Effekt der körperlichen Funktionsfähigkeit auf die SDNN nicht mehr nachzuweisen. Da sich in der Gruppe mit bear- 
beiteten EKGs signifikant mehr weibliche Probanden befanden, kann auch hier ein Interaktionseffekt des Geschlechts auf das Ergebnis, wie in Unterhypothese D bereits postuliert, erklärend sein (s. 3.2.2.3.4 Unterhypothese D, S.61). Ansonsten sind weitere, z.B. auch herzrhythmische, Faktoren, die die beiden Kollektive unbearbeitete und bearbeitete EKGs unterschieden, möglich. Für die explorative Analyse ohne Probanden mit Betablocker war wieder ein signifikanter Effekt der körperlichen Funktionsfähigkeit auf die SDNN nachzuweisen (s. S.71).

Der hypothesenkonforme Einfluss der körperlichen Funktionsfähigkeit auf die SDNN scheint ein nur von ausgewählten somatischen Variablen beeinflusster Zusammenhang zu sein.

Für Taktatmungs- und Ruhephase zeigten sich signifikante hypothesenkonforme Zusammenhänge zwischen dem Ausmaß der körperlichen Funktionsfähigkeit und LF sowie Total-HRV.

Für LF zeigte sich in der Taktatmungs- und in der Ruhephase ein signifikanter konkordanter Effekt für das Ausmaß der körperlichen Funktionsfähigkeit. Es waren also bei abnehmender körperlicher Funktionsfähigkeit sinkende LF-Werte zu beobachten. In der Taktatmungsphase wäre ein Anstieg des LF erwartet worden (s. 1.3.3.2 Atmung, S.10). Hier scheint abnehmende körperliche Funktionsfähigkeit mit einer verminderten Resonanzfähigkeit oder aber auch lediglich einer mangelnden Kooperation bei der Taktatmung einherzugehen. Auch in der Ruhephase zeigte sich ein Zusammenhang zwischen sinkendem Ausmaß der körperlichen Funktionsfähigkeit und abnehmender LF. In der Untersuchung auf den Einfluss somatischer Parameter auf die LF zeigte sich für die Taktatmungs- und Ruhephase in diesem Kollektiv ein klarer Zusammenhang mit verschiedenen somatischen Parametern, wobei für steigendes Alter, abnehmende Gehteststrecke, sowie steigendes NT-pro-BNP und vorhandene Vorerkrankung DM und nur in der Taktatmungsphase für zunehmendes Gewicht sinkende Werte für LF $\mathrm{zu}$ verzeichnen waren (s. Tab. 14 und 15, S.63ff). Für steigendes Alter und DM ist ein Zusammenhang mit sinkender HRV beschrieben (RAJENDRA ACHARYA et al. 2006), auch für Metabolisches Syndrom mit unter anderem steigendem Gewicht (TENTOLOURIS et al. 2008). Hier zeigt sich der deutliche Einfluss somatischer Variablen auf die LF. Im Rahmen der Hypothesenprüfung war dennoch zumindest ein teilweise unabhängiger Effekt der körperlichen Funktionsfähigkeit auf die LF nachzuweisen, insbesondere kein Einfluss des NT-proBNP (s. 3.2.2.3.2 LF, S.58). Dieser Effekt der körperlichen Funktionsfähigkeit auf die LF war auch in der geschlechtergetrennten Analyse stabil (s. 3.2.2.3.4 Unterhypothese D, S.61). 
In der explorativen Analyse war der Effekt der körperlichen Funktionsfähigkeit auf die LF weiter nachvollziehbar (s. S. 69 und 71).

Der hypothesenkonforme Effekt der körperlichen Funktionsfähigkeit auf die LF scheint zwar ein realer Effekt der körperlichen Funktionsfähigkeit zu sein, von einzelnen somatischen Parametern beeinflusst, aber nicht von NT-pro-BNP. Für die Taktatmungsphase liegt möglicherweise eine verminderte Resonanzfähigkeit der Probanden mit niedriger körperlicher Funktionsfähigkeit vor. Wahrscheinlicher ist allerdings für beide Phasen eine niedrige LF bei niedriger körperlicher Funktionsfähigkeit aufgrund der interagierenden somatischen Erkrankungen.

Für den globalen HRV-Parameter „Total“ zeigt sich in der Taktatmungs- und in der Ruhephase eine hypothesenkonforme Absenkung der Werte bei Verschlechterung der körperlichen Funktionsfähigkeit. In der Untersuchung auf den Einfluss somatischer Parameter auf die Total-HRV zeigte sich für die Taktatmungs- und Ruhephase in diesem Kollektiv (s. Tab. 14 und 15, S.63ff) ein sinkender Total-Wert für steigendes Alter, steigenden BMI und vorhandene Vorerkrankung DM. Dies entspricht den bekannten Zusammenhängen (RAJENDRA ACHARYA et al. 2006), auch für Metabolisches Syndrom mit unter anderem steigendem Gewicht (TENTOLOURIS et al. 2008). Auch für abnehmende Länge der Gehstrecke zeigte sich ein klarer Zusammenhang mit abnehmendem Maß an körperlicher Funktionsfähigkeit (s.Tab. 16, S.66). Nur in der Taktatmungsphase zeigte sich weiterhin eine erhöhte Total-HRV in der Analyse für Hypothese 3 bei weiblichen Probanden (s. Tab.15, S.64 und Tabelle 8, S.41). Bezüglich der einflussnehmenden somatischen Faktoren auf die Total-HRV zeigte sich eine deutliche Ähnlichkeit zu den Einflussfaktoren für LF. Im Rahmen der Hypothesenprüfung war zumindest ein teilweise unabhängiger Effekt der körperlichen Funktionsfähigkeit auf die TotalHRV nachzuweisen (s. 3.2.2.3.3 Total, S.59). Dieser Effekt der körperlichen Funktionsfähigkeit auf die Total-HRV war auch in der geschlechtergetrennten Analyse nur für Männer nachweisbar (s. 3.2.2.3.4 Unterhypothese D, S.61).

In der explorativen Analyse war der Effekt der körperlichen Funktionsfähigkeit auf die TotalHRV in der Analyse ohne bearbeitete EKGs nicht nachweisbar. Auch hier ist, analog der Veränderungen für LF, eine mögliche Interaktion des Geschlechts beziehungsweise aber auch herzrhythmischer Faktoren möglich. Die Untergruppe „bearbeitete EKGs“ zeigte deutlich mehr weibliche Probanden und das EKG war aufgrund arrhythmischer Auffälligkeiten bearbeitet worden (s. 2.5.2, S.29 und 3.2.6.1 Explorative Analyse nur unbearbeitete EKGs, ab S. 
67). Für die Untersuchung von nur Probanden ohne Betablocker war der Effekt der körperlichen Funktionsfähigkeit auf die Total-HRV weiter nachvollziehbar (s. 3.2.6.2.4, S.71)

Der hypothesenkonforme Einfluss der körperlichen Funktionsfähigkeit auf die Total-HRV scheint von einigen somatischen Variablen beeinflusst zu sein, ähnlich dem Zusammenhang mit LF. Auch hier ist kein Einfluss des NT-pro-BNP zu verzeichnen.

Zusammenfassend scheint der Zusammenhang von niedriger körperlicher Funktionsfähigkeit und erniedrigter SDNN, LF und Total nur von einigen wenigen somatischen Faktoren beeinflusst und daher offensichtlich ein zu den somatischen Faktoren zusätzlicher Effekt der selbst wahrgenommenen körperlichen Funktionsfähigkeit auf die aufgeführten HRV-Parameter zu sein.

Insgesamt kann in dieser großen Analyse von HRV an N= 468 Probanden mit kardiovaskulären Risikofaktoren und zum Teil kardialen Erkrankungen für zunehmende körperliche Funktionsfähigkeit in der Taktatmungs- und in der Ruhephase eine autonome Dysfunktion im Sinne einer reduzierten vagalen Kontrolle im Sinne von abnehmenden HRV-Parametern (SDNN, LF und Total ) nachgewiesen werden.

Für HRV und Lebensqualität wurden nur wenige Studien gefunden (s. 1.3.5 Psychische Vorerkrankungen, S.12). In den vorliegenden Studien waren hohe Werte für körperliche Lebensqualität mit höheren HRV Parametern verbunden gewesen (VANGESTEL et al. 2011; HATHAWAY et al. 2000).

Auch in der vorliegenden Studie zeigte sich ein Zusammenhang zwischen niedriger körperlicher Funktionsfähigkeit als Parameter der Lebensqualität mit erniedrigten HRV-Parametern, allerdings nur in der Taktatmungs- und in der Ruhephase.

In der Gehphase zeigte sich analog zu den Veränderungen für Depressivität und Vitale Erschöpfung eine Vagusdominanz und Sympathikusminderinnervation. Dies ist möglicherweise bei dem somatisch betonten Parameter körperliche Funktionsfähigkeit bedingt durch tatsächliche körperliche Einschränkung oder durch erlernte körperliche Zurückhaltung bei belastungsabhängigen Beschwerden oder wie auch für Depressivität und Vitale Erschöpfung vermutet motivationsbedingte Minderaktivität im Rahmen des Gehtests. 
Zusammenfassend unterstützen die erhobenen Ergebnisse den bereits in anderen Arbeiten beobachteten Zusammenhang zwischen abnehmender körperlicher Funktionsfähigkeit und erniedrigten HRV-Parametern in dieser Arbeit im Sinne erniedrigter Werte für SDNN in der Taktatmungsphase, LF und Total in Taktatmungs- und Ruhephase.

Zusammenfassend zeigten sich für abnehmende selbst wahrgenommene körperliche Funktionsfähigkeit, als Teilaspekt der Lebensqualität, Zeichen einer autonomen Dysfunktion für die Herzfrequenzvariabilitätsparameter SDNN in der Taktatmungsphase sowie für LF und Total in der Taktatmungs- und Ruhephase.

Für Depressivität, Angst und Vitale Erschöpfung war in keiner der Untersuchungsphasen eine autonome Imbalance nachweisbar.

\subsection{Methodikdiskussion}

\subsubsection{Langzeit-EKG}

\subsubsection{Experimentelles Setting}

Insbesondere in der Gehphase konnte kein eindeutiger steady state erreicht werden. Im Rahmen der Auswertung der EKGs ist automatisch ein lineares Detrending erfolgt. Manuell wurde im Sinne des Erreichens einer stabilen Untersuchungsphase die Gehphase in 2-MinutenPhasen unterteilt. Insbesondere die LF ist bei zu kurzen Messungen kritisch zu bewerten. Die Untersuchungsphase von 2 Minuten schien akzeptal zu sein. Möglicherweise wäre ein noch weiteres, komplexeres Detrending bei zukünftigen Studien mit ähnlichem Setting sinnvoll. Im Rahmen des experimentellen Settings war kein Stresstest zur Provokation einer angstbesetzten Situation integriert, dies könnte in zukünftigen Studien ergänzt werden. 


\subsubsection{Aufnahmequalität}

$\mathrm{Zu}$ kommentieren sind bezüglich der Langzeit-EKG-Daten die Auffälligkeiten im Vergleich der bearbeiteten mit den unbearbeiteten EKGs (s. 6.6 T-Testung bearbeiteter und unbearbeiteter EKGs, S.125). Dies wurde im Rahmen der explorativen Analysen beachtet (s. 3.2.6.1 Nur unbearbeitete EKGs, ab S.67).

Eine doppelte Messung der Probanden wäre nützlich zur Elimination technisch schlechter oder artefaktreicher EKGs.

\subsubsection{Atemkontrolle}

In einigen Studien wurde eine Kontrolle der Atemfrequenz, aufgrund der Atemabhängigkeit einzelner Parameter, durchgeführt. Auch dies bleibt bei weiteren Studien zu diskutieren.

\subsubsection{Technische Aspekte}

Die Speicherung der Daten auf CDs scheint störanfällig zu sein. Vermutlich sind hier einige Datensätze verloren gegangen. Eine verlässlichere Sicherung ist zum Beispiel mit externen Festplatten möglich.

Die verwendete EKG-Analyse-Software besitzt keine automatische Platzierung des Kursors am Markierungspunkt. Dies erfolgte manuell. Fehler im Millisekundenbereich sind dadurch möglich, aber klinisch unerheblich.

\subsubsection{Psychische Daten}

Bezüglich der Fragebogendaten ist insbesondere zu erwähnen, dass fehlende Daten das Bild verzerren können und vermutlich eher positiver ausgefüllt, als tatsächlich empfunden wurde. Vielfach füllten die Patienten nur Teile des Fragebogens aus. Im Verlauf des KNHI-Projekts wurde daraufhin ein gekürzter Fragebogen erstellt.

\subsubsection{Gehtest}

Ein Versuchsleitereffekt ist im Rahmen der EKG-Daten-Erhebung und dem 6-MinutenGehtest bei drei unterschiedlichen Untersuchern nicht auszuschließen. 


\subsection{Limitationen dieser Studie}

Insbesondere durch die bei kardialen Probanden häufigen Arrhythmien mussten einige Probanden ausgeschlossen werden. Die Vormedikation mit Betablocker hat einen starken Einfluss auf die HRV und schränkt die Beurteilbarkeit bei kardialen Patienten allein aufgrund der Häufigkeit der Anwendung dieser Medikation in diesem Gebiet stark ein.

\subsection{Ausblick/weitere Forschung}

Die Klärung der Uneinheitlichkeit der Befunde bezüglich der untersuchten psychischen Auffälligkeiten ist nicht abschließend erreicht. Weitere Forschung sollte die weiteren Aspekte der Lebensqualität des SF-36 betrachten, Angstsymptomatik möglichst durch einen Stresstest auslösen, und das Kollektiv sollte möglichst keine Betablocker einnehmen. Allerdings sind auch andere Medikamente, wie Antiarrhythmika, aber auch einige Psychopharmaka wie z. B. tricyclische Antidepressiva und Antihistaminika mit arrhythmisierenden Wirkungen assoziiert worden (TASK FORCE OF THE EUROPEAN SOCIETY OF CARDIOLOGY AND THE NORTH AMERICAN SOCIETY OF PACING AND ELECTROPHYSIOLOGY 1996; ROTTENBERG 2007). Zur Optimierung der LZ-EKG-Aufnahmequalität sollte eine bessere Stabilität der Aktivitätsphase, z.B. mittels Fahrradergometrie, erprobt werden. Die Zusammenhänge insbesondere des NT-pro-BNP mit erhöhten HRV-Parametern sollte näher beleuchtet werden. Insbesondere da, wie in den vorangegangenen Kapiteln erläutert, nicht nur ein Einfluss auf die Herzinnervation, sondern auch Zusammenhänge mit einzelnen psychosomatischen Variablen beschrieben werden (s. 1.4.1 NT-pro-BNP, ab S. 13). 


\section{Zusammenfassung}

\section{Einleitung:}

Die psychosomatischen Parameter „Depressivität“, „Angst“, „Vitale Erschöpfung“ und „selbst empfundene Lebensqualität“ interferieren mit Auftreten, Verlauf und Prognose von kardialen Erkrankungen. Als ursächlich für diesen Zusammenhang wird unter anderem eine Imbalance des autonomen Nervensystems im Sinne einer autonomen Dysfunktion diskutiert. Die Funktion des autonomen Nervensystems kann mittels Herzfrequenzanalyse sichtbar und quantifizierbar gemacht werden. Erwartet, und in einigen Studien zumeist bei körperlicher Ruhe nachgewiesen, wurde eine autonome Dysfunktion mit Dominanz des Sympathikus und Supprimierung des Parasympathikus bei psychischer Belastung zum Beispiel durch Depressivität. Allerdings sind die bisherigen Studienergebnisse bezüglich des Zusammenhangs psychosomatischer Belastung und Herzfrequenzvariabilität uneinheitlich. Die vorliegende Studie untersucht hauptsächlich, ob die Uneinheitlichkeit der Ergebnisse durch die unterschiedlichen Messsituationen begründet ist.

\section{Methoden:}

Von 468 Probanden des Kompetenznetzes Herzinsuffizienz in Göttingen wurden LangzeitEKGs in drei verschiedenen autonomen Aktivierungszuständen (Geh-, Taktatmungs- und Ruhephase) erstellt und die Herzfrequenzvariabilität in diesen Phasen analysiert. Mittels psychologischer Fragebögen (HADS, Maastricht-Fragebogen, SF-36) wurden die Probanden bezüglich Depressivität, Angst, Vitaler Erschöpfung und selbst berichteter körperlicher Funktionsfähigkeit (Teilaspekt der Lebensqualität) beurteilt. Primär wurde der Einfluss der vier genannten psychosomatischen Variablen auf die Herzfrequenzvariabilität untersucht. Zusätzlich wurde der Zusammenhang zwischen kardialen Risikofaktoren, Erkrankungen, Schweregradmarkern (unter anderem Ejektionsfraktion und NT-pro-BNP) und der Herzfrequenzvariabilität einerseits und den psychosomatischen Variablen andererseits näher beleuchtet. 


\section{Ergebnisse:}

Für die vier untersuchten psychosomatischen Variablen waren in den unterschiedlichen Messsituationen nur wenige signifikante Zusammenhänge mit den HerzfrequenzvariabilitätsParametern nachweisbar.

Für Angst war in keiner der Untersuchungsphasen eine signifikante Veränderung der Herzfrequenzvariabilitätsparameter festzustellen. Für Depressivität und Vitale Erschöpfung zeigten sich keine Zeichen der autonomen Dysfunktion, aber dafür Zeichen einer mangelnden Motivation, insbesondere im Rahmen des Gehtests. Für als niedrig empfundene körperliche Funktionsfähigkeit zeigte sich in der Gehphase und für den Herzfrequenzvariabilitätsparameter LF/HF in allen Untersuchungsphasen eine ähnliche Konstellation der Herzfrequenzvariabilitätsparameter wie für Depressivität und Vitale Erschöpfung in der Gehphase. Als Erklärung sollte neben Motivation auch eine somatische Ursache dieser Veränderungen für körperliche Funktionsfähigkeit diskutiert werden. In der Taktatmungs- und Ruhephase zeigte sich für niedrig empfundene körperliche Funktionsfähigkeit ein klarer Zusammenhang mit erniedrigten Herzfrequenzvariabilitätsparametern (SDNN, LF, Total-Herzfrequenzvariabilität).

Von den somatischen Faktoren war für NT-pro-BNP ein besonders bemerkenswerter Zusammenhang mit den psychosomatischen Variablen beobachtet worden. Sowohl für hohe Vitale Erschöpfung als auch für als niedrig empfundene körperliche Funktionsfähigkeit bestand in der vorliegenden Studie ein klarer Zusammenhang mit hohen Werten für NT-pro-BNP.

\section{Diskussion:}

Zusammenfassend ist festzuhalten, dass die erwartete autonome Dysfunktion für Patienten mit erhöhter Depressivität, Angst und Vitaler Erschöpfung in allen drei untersuchten Aktivierungszuständen des autonomen Nervensystems entgegen der vorherrschenden Expertenmeinung nicht nachzuweisen war.

Für abnehmende, selbst wahrgenommene körperliche Funktionsfähigkeit als Teilaspekt der Lebensqualität ließen sich Zeichen einer autonomen Dysfunktion mit erniedrigten Herzfrequenzvariabilitätsparametern SDNN in der Taktatmungsphase sowie LF und TotalHerzfrequenzvariabilität in der Taktatmungs- und Ruhephase nachweisen. Diese Veränderungen, die zum Beispiel auch bei kardialen Erkrankungen beschrieben werden, waren trotz der somatischen Betonung des Parameters „körperliche Funktionsfähigkeit“ zumindest teilweise unabhängig von den im Kollektiv erhobenen somatischen Faktoren, insbesondere von NTpro-BNP. 


\section{Anhang}

\subsection{Diastolischer Herzinsuffizienzscore im KNHI}

\begin{tabular}{|c|c|c|c|c|c|}
\hline & $\begin{array}{l}\text { Normal } \\
\quad=0\end{array}$ & $\begin{array}{l}\text { Verzö- } \\
\text { gerte } \\
\text { Relaxa- }\end{array}$ & $\begin{array}{l}\text { Pseudonor- } \\
\text { maler Fül- } \\
\text { lungspatern }\end{array}$ & \multicolumn{2}{|c|}{$\begin{array}{c}\text { Restriktiver } \\
\text { Füllungspatern } \\
=3\end{array}$} \\
\hline & & & & $\begin{array}{l}\text { reversi- } \\
\text { bel }\end{array}$ & $\begin{array}{c}\text { nicht } \\
\text { reversi- } \\
\text { bel }\end{array}$ \\
\hline $\begin{array}{c}\text { E/A } \\
\text { Mitralisdoppler }\end{array}$ & $>1$ & $<1$ & $1-2$ & \multicolumn{2}{|c|}{$>2$} \\
\hline & $\begin{array}{c}\& \\
3 \text { der } 3 \\
\text { folgen- } \\
\text { den } \\
\text { Kriterien } \\
\text { erfüllt }\end{array}$ & & $\begin{array}{c}\& \\
1 \text { der } 3 \text { Krite- } \\
\text { rien erfüllt }\end{array}$ & \multicolumn{2}{|c|}{$\begin{array}{c}\& \\
1 \text { der } 2 \text { folgenden } \\
\text { Kriterien erfüllt und } \\
\text { E/A unter Valsalva- } \\
\text { Manöver }\end{array}$} \\
\hline E/em` & $<10$ & & $>=10$ & \multicolumn{2}{|c|}{$>=10$} \\
\hline $\begin{array}{c}\text { S/D } \\
\begin{array}{c}\text { Pulmonalvenen- } \\
\text { doppler }\end{array}\end{array}$ & $>=1$ & & $<1$ & \multicolumn{2}{|c|}{$<1$} \\
\hline $\begin{array}{c}\text { E/A } \\
\text { (unter Valsalva) }\end{array}$ & $>=1$ & & $<1$ & $<1$ & $>=1$ \\
\hline
\end{tabular}

Tabelle 17 Einteilung der diastolischen Dysfunktion im Kompetenznetz Herzinsuffizienz erhoben nach ASE-Richtlinien (BREMECKER 2010). 


\subsection{Fragebogen KNHI mit Polung}

\section{Abbildung 4 Deckblatt KNHI-Fragebogen}

\begin{tabular}{|c|c|c|c|c|}
\hline & \multicolumn{3}{|c|}{ Befragung zur Lebensqualität } & \\
\hline DiaDEM & Center - ID $ل ـ ل ـ$ & Stud - PID & $\downarrow$ & LEBENSQ \\
\hline Anlass: $O$ Baseline $O F$ & ollow-up 1 (12 Mon.) O Fc & Ilow-up 2 (24 Mon.) & Datum der Befragung & 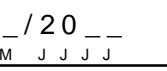 \\
\hline
\end{tabular}

Sehr geehrter Patient, sehr geehrte Patientin,

hiermit überreichen wir Ihnen einige Fragebögen zu Ihrem Gesundheitszustand, Ihrem persönlichen Lebensgefühl und Ihrem seelischen Empfinden, um den Zusammenhang zwischen Herzfunktion und allgemeinem Befinden zu untersuchen.

Selbstverständlich ist die Beantwortung freiwillig und ohne Einfluss auf Ihre Behandlung. Alle Ihre Angaben unterliegen der ärztlichen Schweigepflicht und werden nach den Bestimmungen des Datenschutzgesetzes anonym ausgewertet. Eine Weitergabe an Dritte ist ausgeschlossen.

\section{Bitte beachten Sie beim Ausfüllen:}

- Bei den vorliegenden Bögen handelt es sich um abgeschlossene Fragenkomplexe, so dass es sich nicht vermeiden ließ, dass sich einige Fragen ähneln oder wiederholen. Wir bitten Sie dennoch, alle Fragen vollständig mit jeweils einem Kreuz zu beantworten. Solten Sie bei einer Frage Zweifel haben, kreuzen Sie bitte die Antwort an, die noch am ehesten für Sie zutrifft.

- Bei der Beantwortung können Sie sich ruhig Zeit lassen. Allerdings sollten Sie nicht ins Grübeln geraten, sondern eher spontan antworten.

- Selbstverständlich ist es unbedingt notwendig, dass Sie die Fragen selbst beantworten und sich dabei z.B. nicht "helfen“ oder gar „vertreten“ lassen. Hilfe ist auch gar nicht möglich, da es bei den Fragen um Ihre ganz persönlichen Empfindungen geht, die nur Sie allein kennen.

Wir bedanken uns herzlich für Ihre Mitarbeit!

Ihr Studienteam

\section{Ansprechpartnerin für Rückfragen:}

Dipl. Psych. Birgit Büchner

Universität Göttingen, Abt. Klinik und Poliklinik für Psychosomatik und Psychotherapie von-Siebold-Str. 5, 37075 Göttingen; Tel.: 0551/39-14354 
Abbildung 5 SF-36, Seite 1 KNHI-Fragebogen; die großen Kästchen entsprechen der Richtung der Polung.

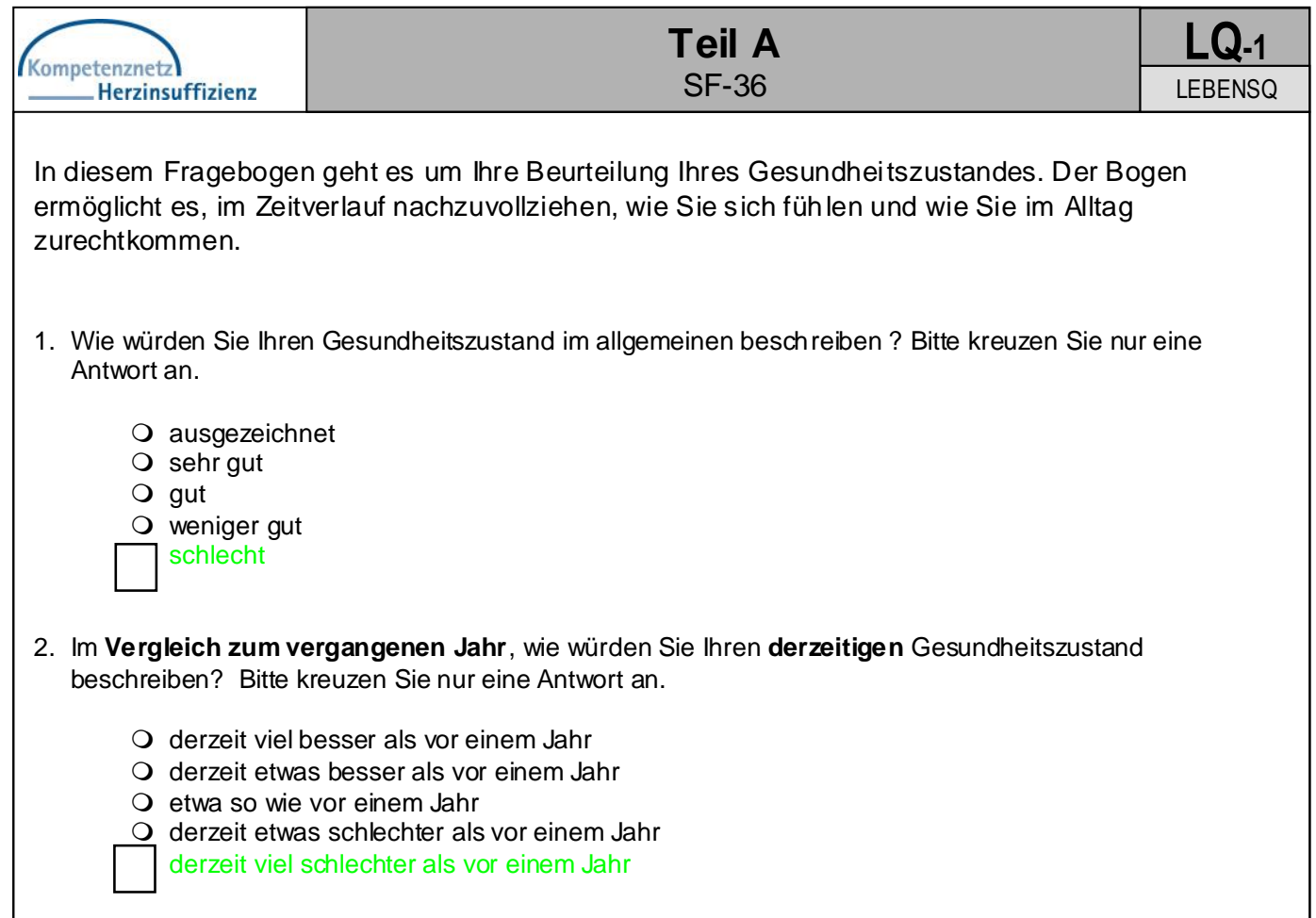

3. Im folgenden sind einige Tätigkeiten beschrieben, die Sie vielleicht an einem normalen Tag ausüben. Sind Sie durch Ihren derze itigen Gesundheitszustand bei diesen Tätigkeiten eingeschränkt? Wenn ja, wie stark? Bitte kreuzen Sie in jeder Zeile nur eine Antwort an.

\begin{tabular}{lccc}
\hline $\begin{array}{l}\text { Anstrengende Tätigkeiten, z.B. schnell laufen, schwere } \\
\text { Gegenstände heben, anstrengenden Sport treiben }\end{array}$ & $\begin{array}{c}\text { ja, stark ein- } \\
\text { geschränkt }\end{array}$ & $\begin{array}{l}\text { ja, etwas ein- } \\
\text { geschränkt }\end{array}$ & $\begin{array}{c}\text { nein, gar nicht } \\
\text { eingeschränkt }\end{array}$ \\
\hline $\begin{array}{l}\text { Mittelschwere Tätigkeiten, z.B. einen Tisch verschieben, } \\
\text { Staubsaugen, Kegeln, Golf spielen }\end{array}$ & & 0 & 0 \\
\hline Einkaufstaschen heben oder tragen & & 0 & 0 \\
\hline Mehrere Treppenabsätze steigen & & 0 & 0 \\
\hline Einen Treppenabsatz steigen & & 0 & 0 \\
\hline Sich beugen, knien, bücken & & 0 & 0 \\
\hline Mehr als 1 Kilometer zu Fuß gehen & & 0 & 0 \\
\hline Mehrere Straßenkreuzungen weit zu Fuß gehen & & 0 & 0 \\
\hline Eine Straßenkreuzung weit zu Fuß gehen & & 0 & 0 \\
\hline Sich baden oder anziehen & & 0 & 0 \\
\hline
\end{tabular}


Abbildung 6 SF-36, Seite 2 KNHI-Fragebogen; die großen Kästchen entsprechen der Richtung der Polung.

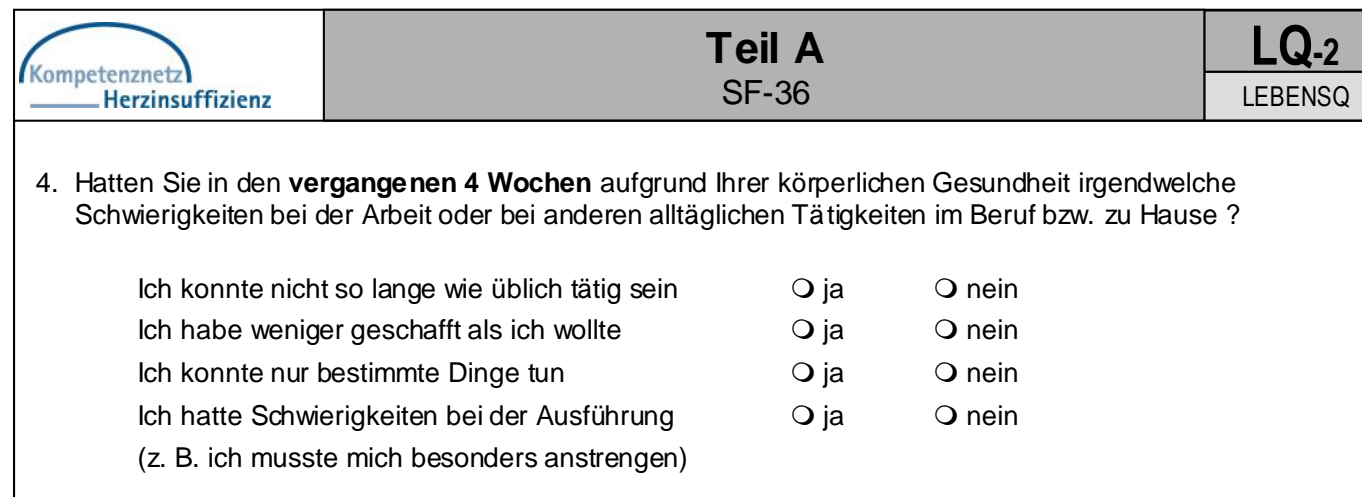

5. Hatten Sie in den vergangenen 4 Wochen aufgrund seelischer Probleme irgendwelche Schwierigkeiten bei der Arbeit oder anderen alltäglichen Tätigkeiten im Beruf bzw. zu Hause (z.B. weil Sie sich niedergeschlagen oder ängstlich fühlten)?
Ich konnte nicht so lange wie üblich tätig sein
O ja O nein
Ich habe weniger geschafft als ich wollte
$O$ ja O nein
Ich konnte nicht so sorgfältig wie üblich arbeiten
O ja O nein

6. Wie sehr haben Ihre körperliche Gesundheit oder Ihre seelischen Probleme in den vergangenen 4 Wochen Ihre normalen Kontakte zu Familienangehörigen, Freunden, Nachbarn oder zum Bekanntenkreis beeinträchtigt?
überhaupt nicht
O etwas
O mäßig
O ziemlich
$\square$ sehr

7. Wie stark waren Ihre Schmerzen in den vergangenen 4 Wochen?
O Ich hatte keine Schmerzen
O sehr leicht
O leicht
O mäßig
O stark
$\square$ sehr stark

8. Inwieweit haben die Schmerzen Sie in den vergangenen $\mathbf{4}$ Wochen bei der Ausübung Ihrer Alltagstätigkeiten zu Hause und im Beruf behindert?
Öberhaupt nicht
$O$ ein bisschen
O mäßig
O ziemlich
$\square$ sehr 
Abbildung 7 SF-36, Seite 3 KNHI-Fragebogen; die großen Kästchen entsprechen der Richtung der Polung.

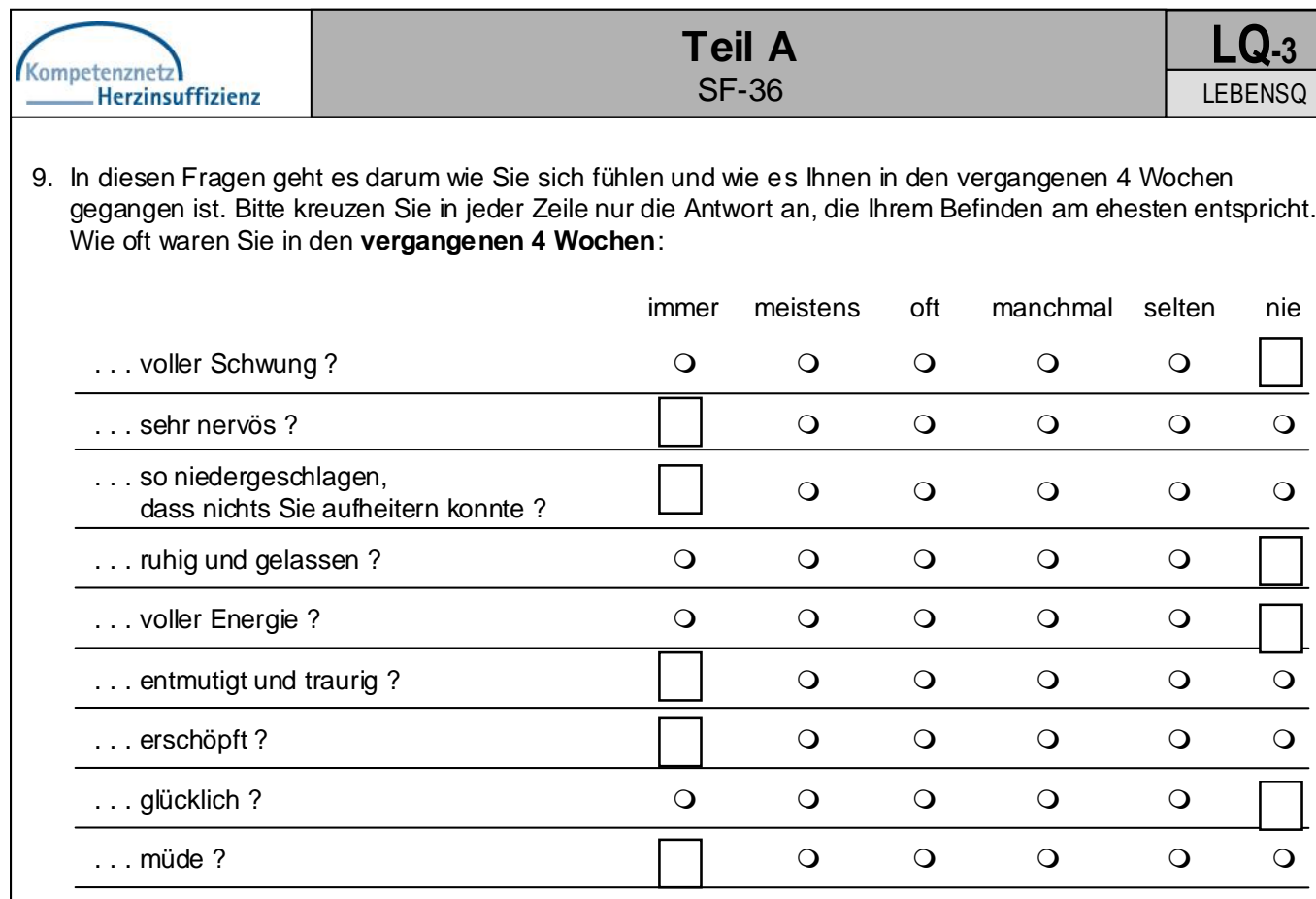

10. Wie häufig haben Ihre körperliche Gesundheit oder seelischen Probleme in den vergangenen 4 Wochen Ihre normalen Kontakte zu anderen Menschen (Besuche bei Freunden, Verwandten usw.) beeinträchtigt?

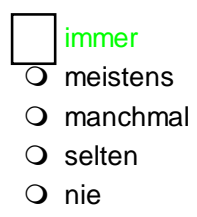

11. Inwieweit trifft jede der folgenden Aussagen auf Sie zu ? Bitte kreuzen Sie in jeder Zeile nur eine Antwort an.

\begin{tabular}{ccccc}
$\begin{array}{c}\text { trifft ganz } \\
\text { zu }\end{array}$ & $\begin{array}{c}\text { trifft weit- } \\
\text { gehend zu }\end{array}$ & $\begin{array}{c}\text { weiß } \\
\text { nicht }\end{array}$ & $\begin{array}{c}\text { trifft weit- } \\
\text { gehend } \\
\text { nicht zu }\end{array}$ & $\begin{array}{c}\text { trifft über- } \\
\text { haupt } \\
\text { nicht zu }\end{array}$ \\
\hline
\end{tabular}

\begin{tabular}{lllll}
\hline $\begin{array}{l}\text { Ich scheine etwas leichter als andere } \\
\text { krank zu werden }\end{array}$ & 0 & 0 & 0 & 0 \\
\hline $\begin{array}{l}\text { Ich bin genauso gesund wie alle } \\
\text { anderen, die ich kenne }\end{array}$ & 0 & 0 & 0 & 0 \\
\hline $\begin{array}{l}\text { Ich erwarte, dass meine Gesundheit } \\
\text { nachlässt }\end{array}$ & $\square$ & 0 & 0
\end{tabular}


Abbildung 8 PHQ-D, Seite 4 KNHI-Fragebogen; die großen Kästchen entsprechen der Richtung der Polung.

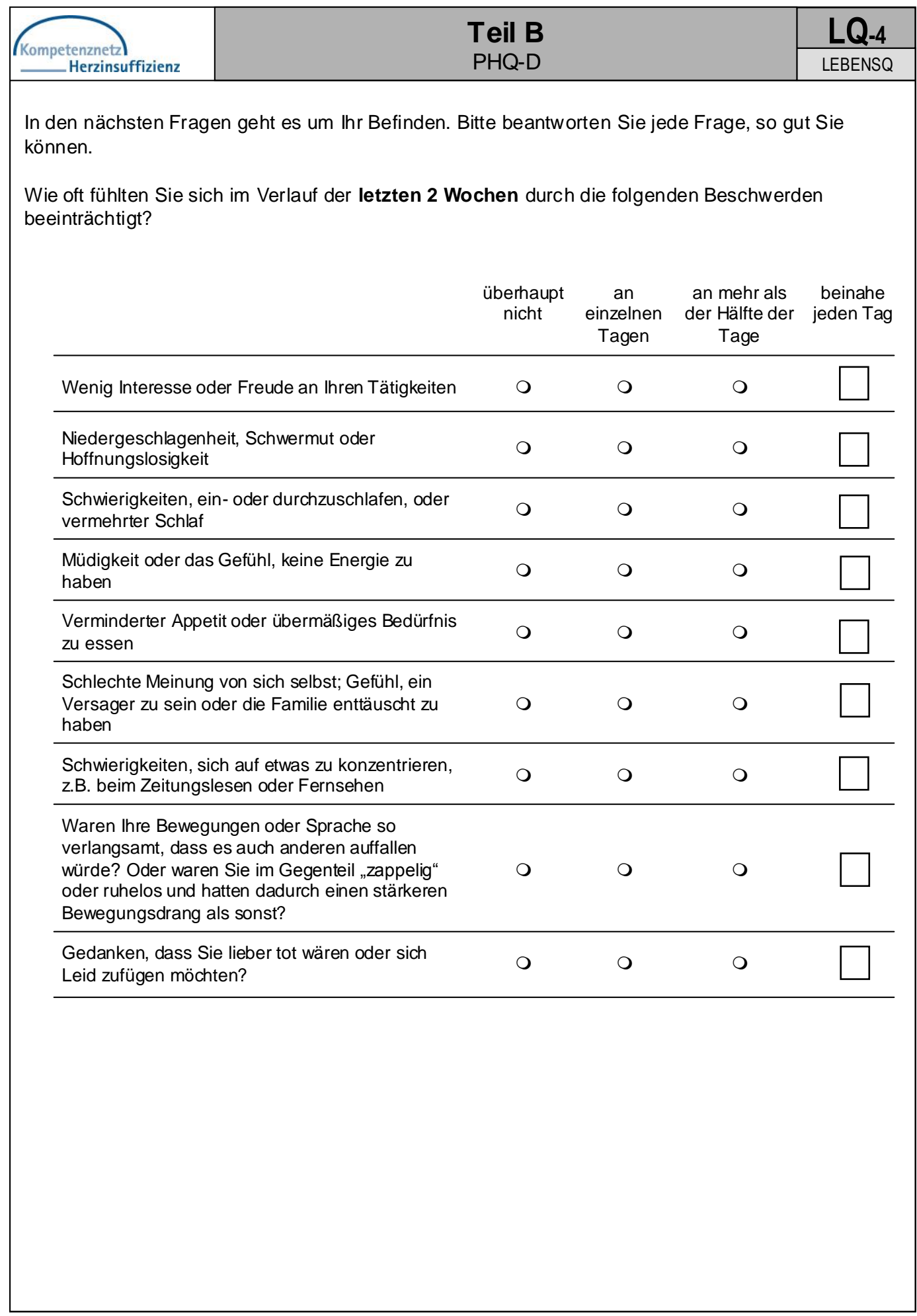


Abbildung 9 Maastricht-Fragebogen, Seite 5 KNHI-Fragebogen; die großen Kästchen entsprechen der Richtung der Polung.

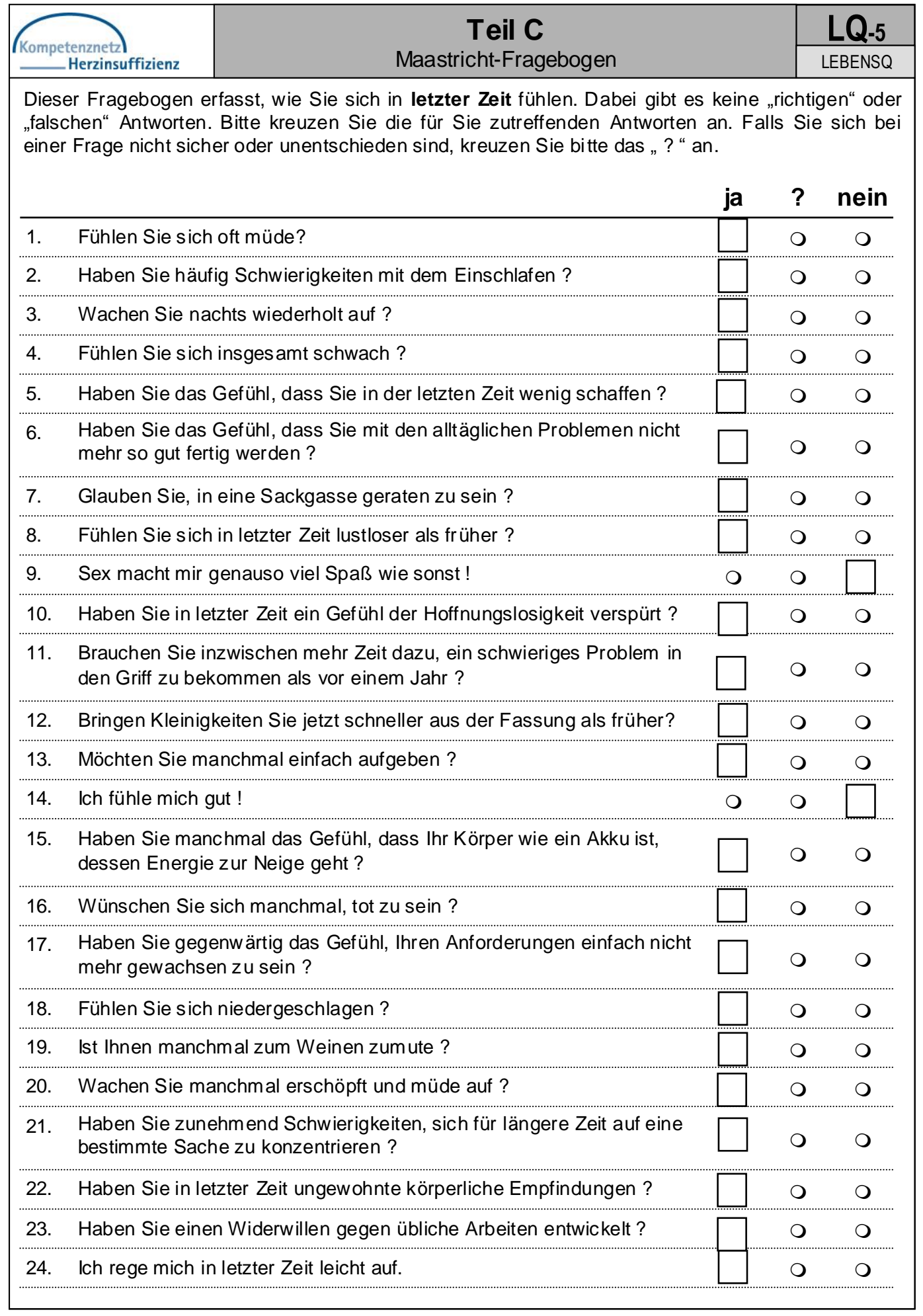


Abbildung 10 HADS, Seite 6 KNHI-Fragebogen; die großen Kästchen entsprechen der Richtung der Polung.

\begin{tabular}{|c|c|c|}
\hline & Teil D & L-6 \\
\cline { 2 - 3 } Kompetenznetz \\
\cline { 3 - 4 } Herzinsuffizienz & HADS & LEBENSQ \\
\hline
\end{tabular}

Bitte beantworten Sie jede der folgenden Fragen so, wie es für Sie persönlich in der letzten Woche am ehesten zutraf. Machen Sie bitte nur ein Kreuz pro Frage und lassen Sie bitte keine Frage aus! Überlegen Sie bitte nicht lange, sondern wählen Sie die Antwort aus, die Ihnen auf Anhieb am zutreffendsten erscheint!

1. Ich fühle mich angespannt oder überreizt
$\square$ meist
$\bigcirc$ oft
O von Zeit zu Zeit /
überhauptnicht gelegentlich

2. Ich kann mich heute noch so freuen wie früher
ganz genau so
nicht ganz so sehr
O nur noch wenig
kaum oder gar nicht

3. Mich überkommt eine ängstliche Vorahnung, dass etwas Schreckliches passieren könnte

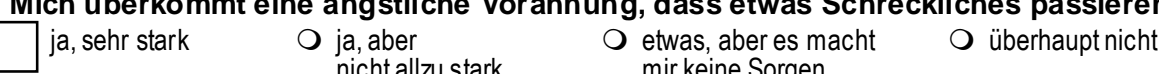

4. Ich kann lachen und die lustige Seite der Dinge sehen
$\bigcirc$ ja, so viel
wie immer
O nicht mehr
ganz so viel
$O$ inzwischen
viel weniger
überhaupt nicht

5. Mir gehen beunruhigende Gedanken durch den Kopf
einen Großteil
$\bigcirc$ verhältnismäßig
O von Zeit zu Zeit, aber nicht allzu oft
O nur gelegentlich / nie

6. Ich fühle mich glücklich
$\square$ überhaupt nicht
$\mathrm{O}$ selten
manchmal
meistens

7. Ich kann behaglich dasitzen und mich entspannen
ja, natürlich
gewöhnlich schon
nicht oft
überhaupt nicht

8. Ich fühle mich in meinen Aktivitäten gebremst
fast immer
$\bigcirc$ sehr oft
manchmal
überhauptnicht

9. Ich habe manchmal ein ängstliches Gefühl in der Magengegend
überhaupt nicht
gelegentlich
ziemlich oft
sehr oft

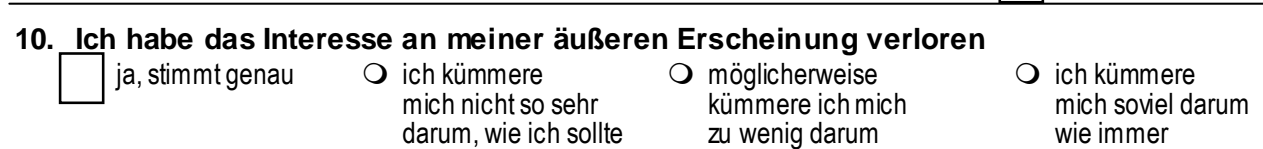

11. Ich fühle mich rastlos, muss immer in Bewegung sein

ja, tatsächlich sehr $\bigcirc$ ziemlich $\bigcirc$ nichtsehr

12. Ich blicke mit Freude in die Zukunft

eherweniger als früher

viel weniger als früher

kaum bis gar nicht

13. Mich überkommt plötzlich ein panikartiger Zustand
ja, tatsächlich
ziemlich oft
nicht sehr oft
überhaupt nicht sehr oft

14. Ich kann mich an eine m guten Buch, einer Radio- oder Fernsehsendung freuen
oft
O manchmal
eher selten
sehr selten 
Abbildung 11 FKV, Seite 7 KNHI-Fragebogen; die großen Kästchen entsprechen der Richtung der Polung.

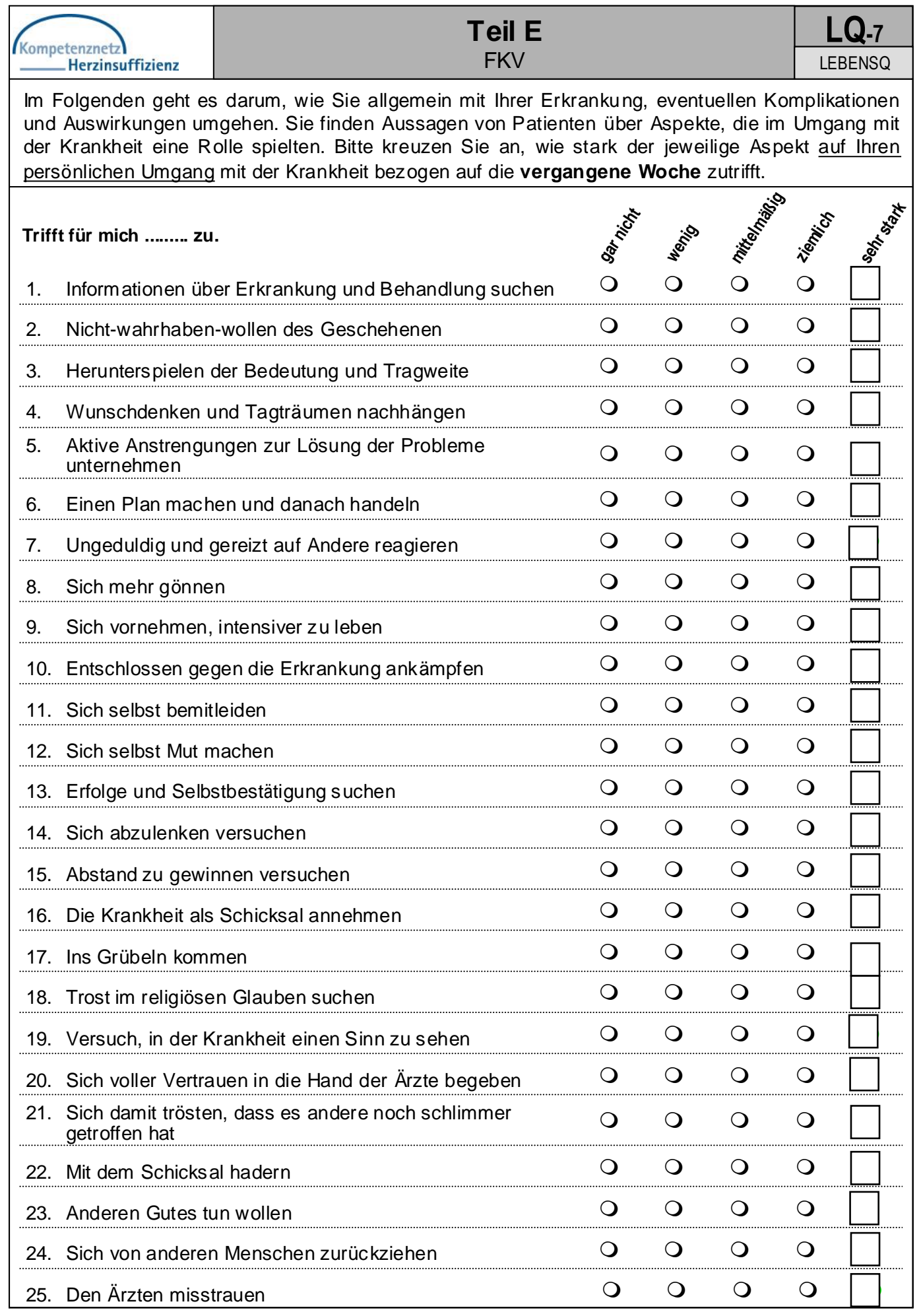


Abbildung 12 ESSI und DS 14, Seite 8 KNHI-Fragebogen; die großen Kästchen entsprechen der Richtung der Polung.

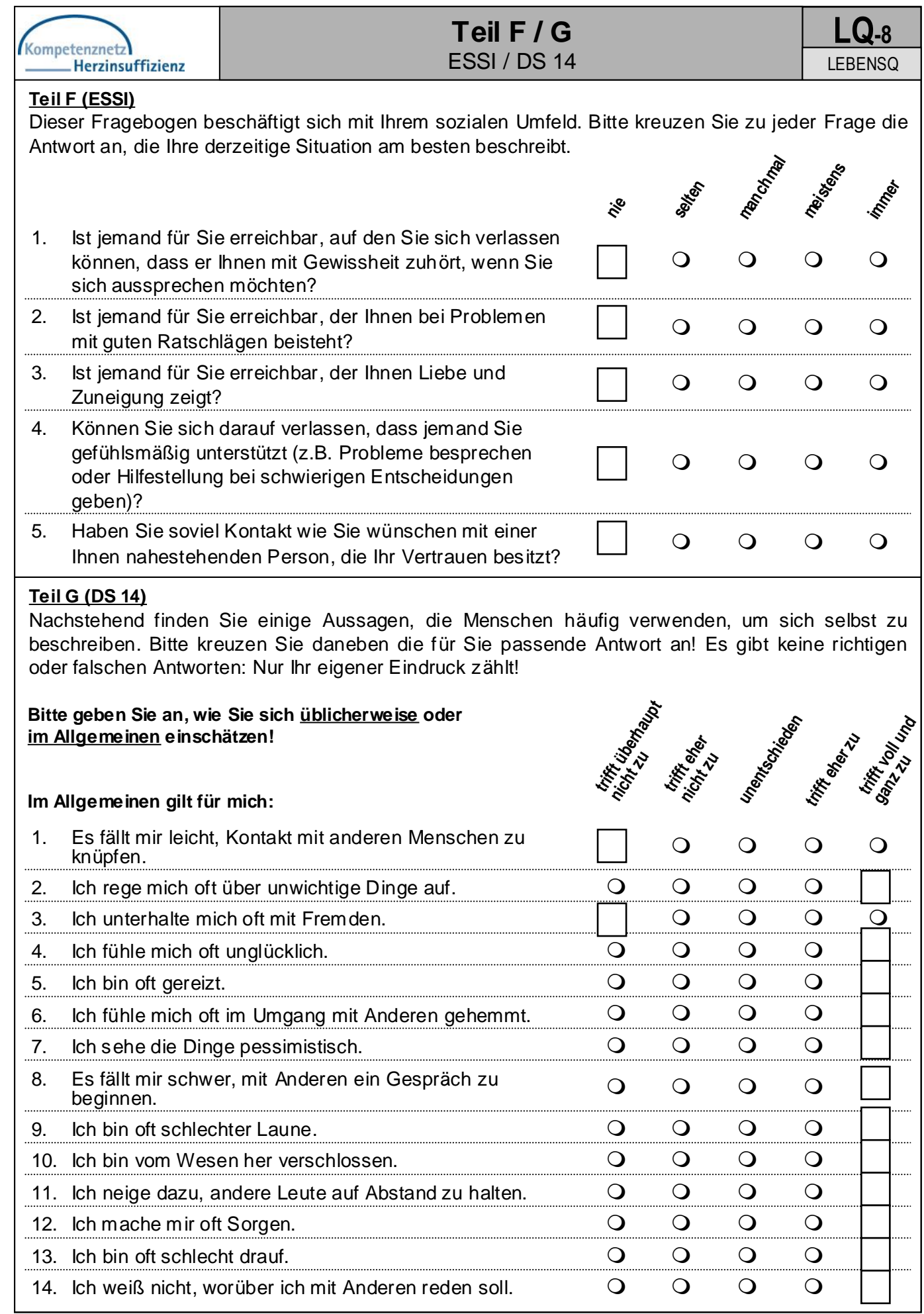


Abbildung 13 GKE und RSQ, Seite 9 KNHI-Fragebogen; die großen Kästchen entsprechen der Richtung der Polung.

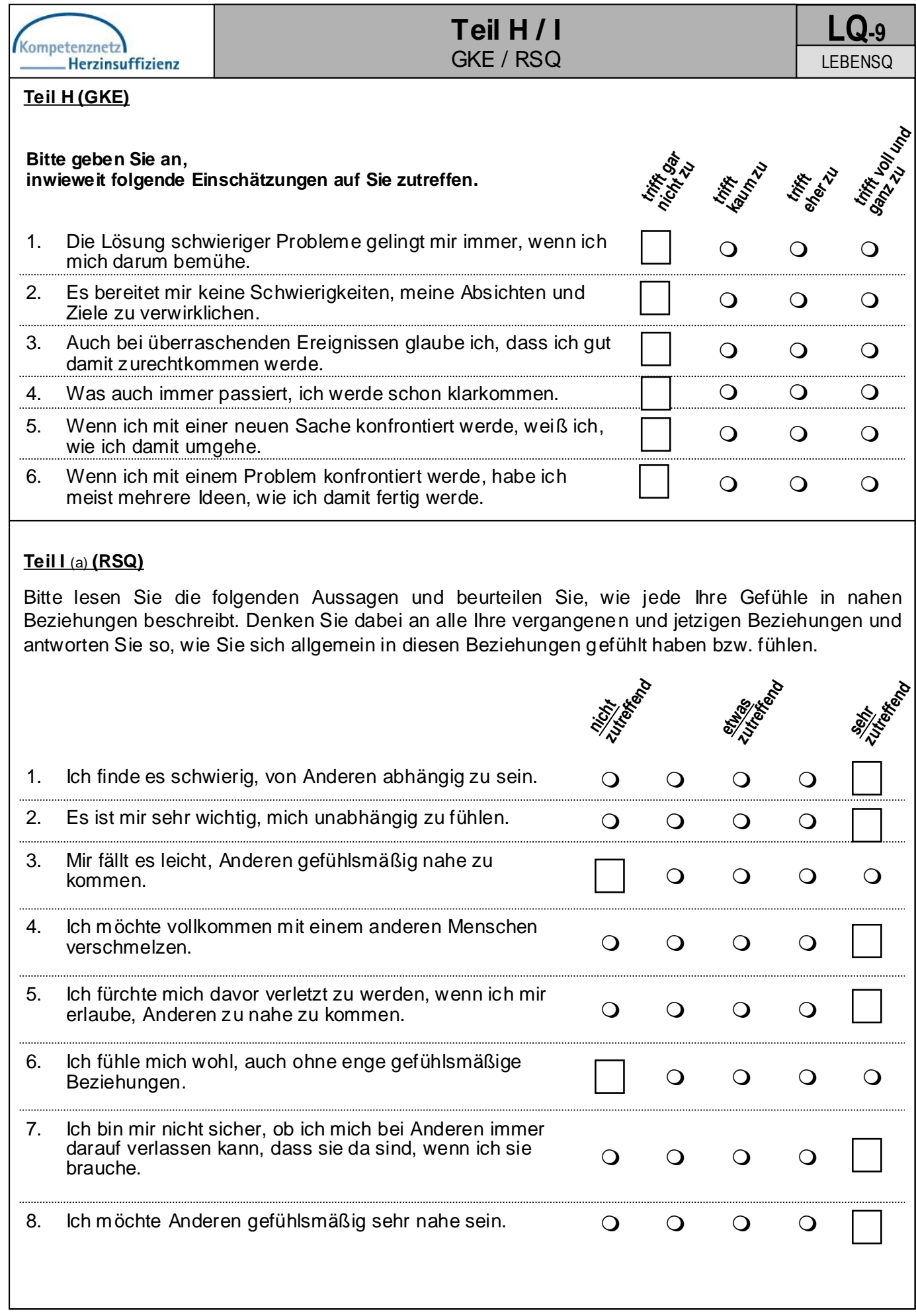


Abbildung 14 RSQ, Seite 10 KNHI-Fragebogen; die großen Kästchen entsprechen der Richtung der Polung.

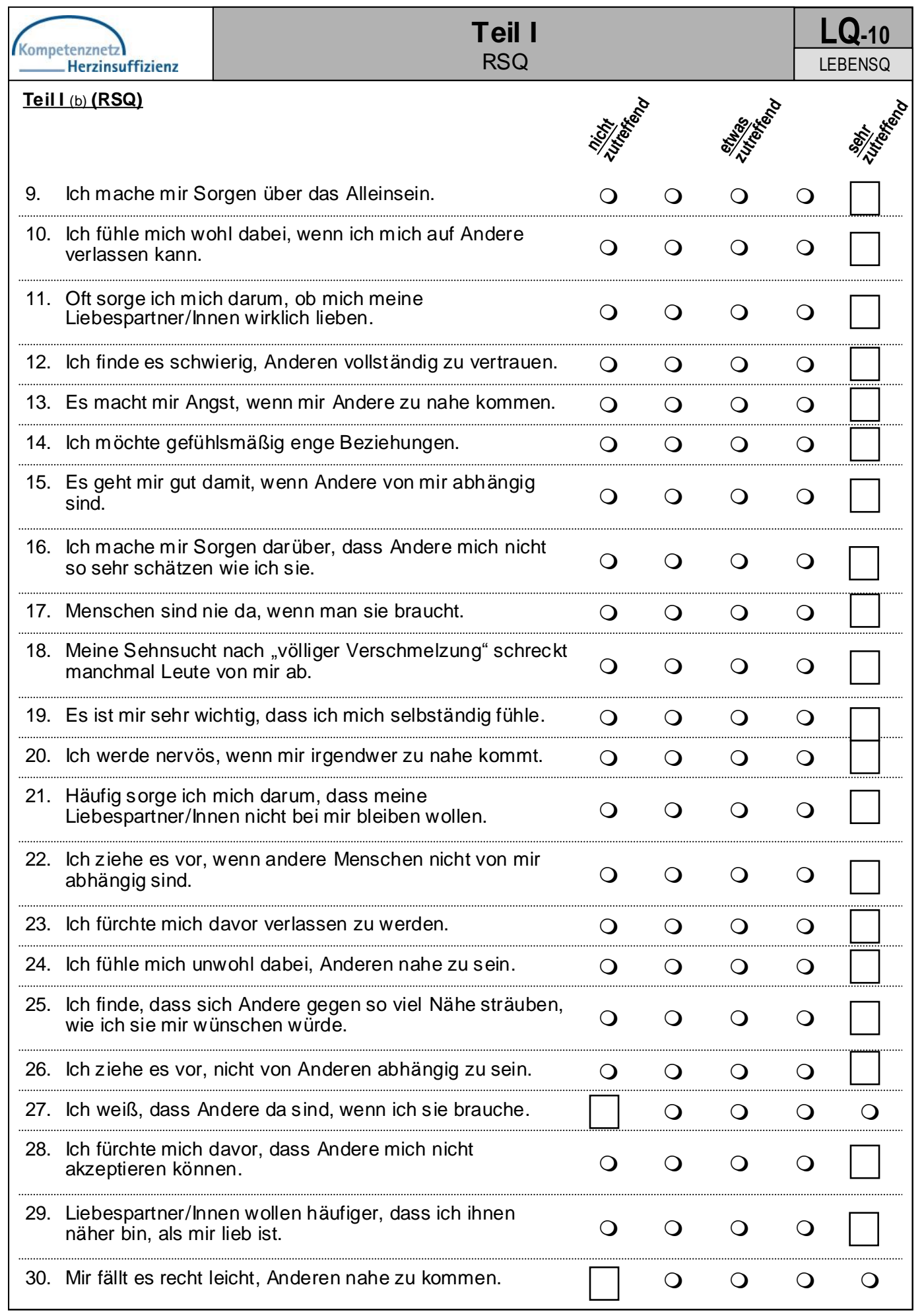


Abbildung 15 MMPI-Z.-Skala, Seite 11 KNHI-Fragebogen

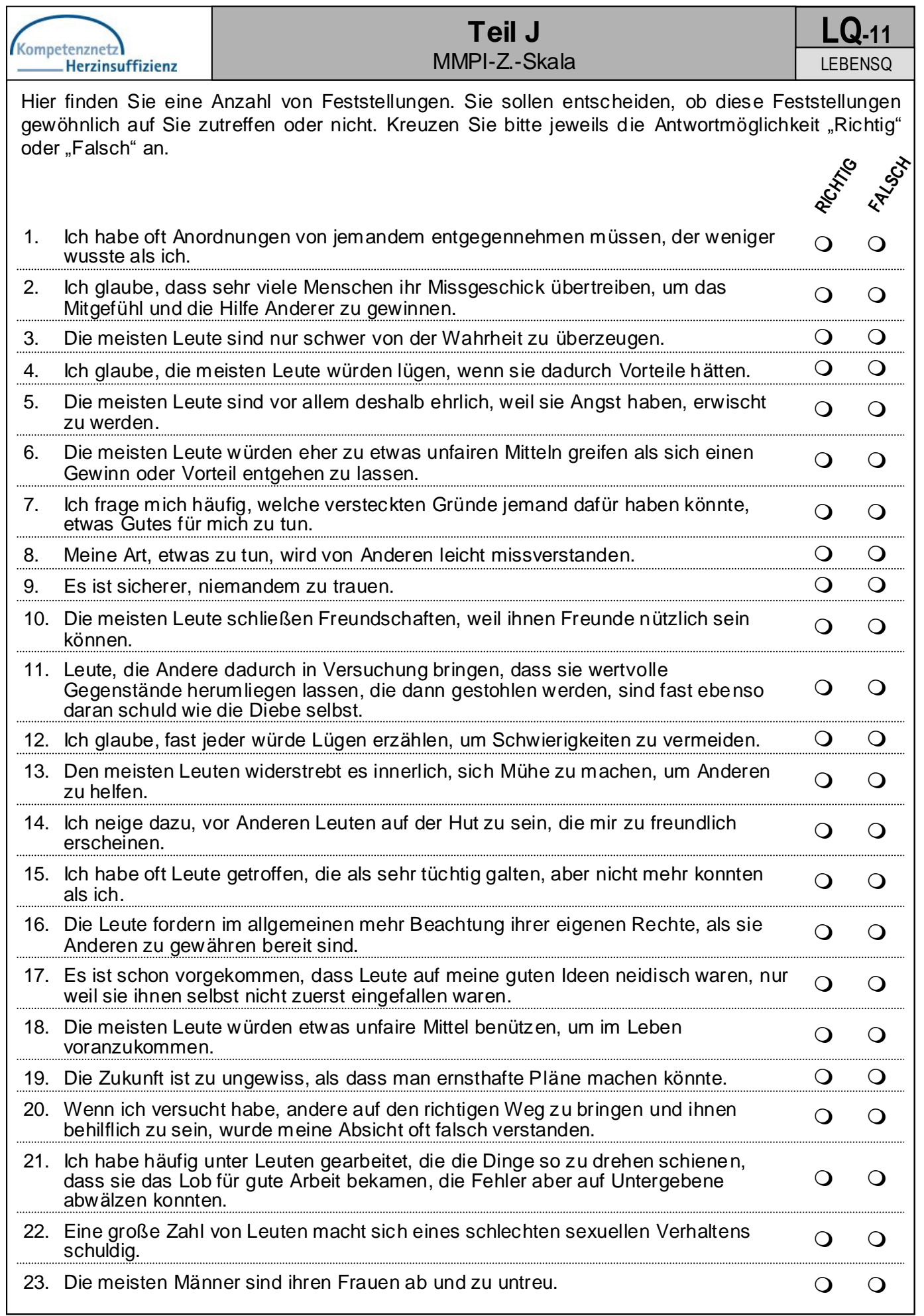


Abbildung 16 Fragen zu DM und Zusatzangaben zur Person, Seite 12 KNHI-Fragebogen

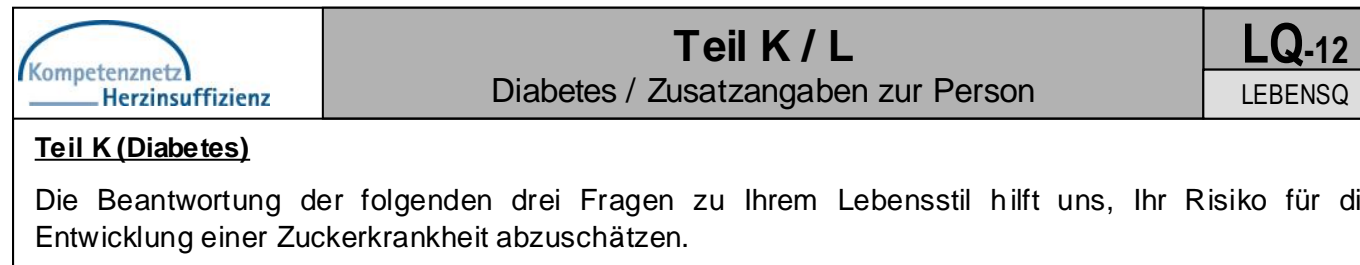

1. Bewegen Sie sich aktiv in Ihrer Freizeit mind. 30 Minuten - die meisten Tage der Woche?

O ja O nein

2. Wie oft essen Sie Gemüse, Früchte oder Beerenfrüchte?

O jeden Tag O nicht jeden Tag

3. Haben Sie Familienangehörige, die jemals mit Diabetes diagnostiziert wurden?

O ja O nein

$\longrightarrow$ wenn,ja' $\square$ Großvater, Großmutter, Onkel, Tante, Cousin, Cousine (nicht Eltern, Geschwister oder eigene Kinder)

leibliche Vater, Mutter, Geschwister oder Kinder

\section{Teil $L$ (a) (Zusatzangaben zur Person)}

1. Welcher Familienstand trifft auf Sie zu?

O verheiratet, mit Ehepartner(in) zusammen lebend

$O$ verheiratet, von Ehepartner(in) getrennt lebend

O ledig

Ogeschieden

$O$ verwitwet

$\longrightarrow$ falls hier angekreuzt: Leben Sie mit eine m Partner / einer Partnerin zusammen?

$$
\text { O ja O nein }
$$

2. Wie viele Personen leben ständig in Ihre $m$ Haushalt, Sie selbst eingeschlossen? (Einschließlich Kinder!)

O 1 Person O mehrere Personen $\rightarrow$ wie viele?

3. Wie viele Personen in Ihrem Haushalt sind 18 Jahre oder älter?

$\downarrow \downarrow$ Personen

4. Welchen höchsten allge meinbildenden Schulabschluss haben Sie?

(Bitte nur den höchsten Abschluss ankreuzen.)

O ohne Schulabschluss

O Hauptschule / Volksschule

O Realschule (Mittlere Reife)

O Polytechnische Oberschule

(vor 1965: Abschluss nach 8.Klasse, ab 1965: Abschluss mit 10. Klasse)

O Fachhochschulreife

O Hochschulreife / Abitur (Gymnasium oder EOS)

$O$ anderer Schulabschluss: 
Abbildung 17 Zusatzangaben zur Person, Seite 13 KNHI-Fragebogen

\begin{tabular}{|c|c|c|}
\hline${ }_{\text {Kompetenznetz }}^{\text {Herzinsuffizienz }}$ & $\begin{array}{c}\text { Teil L } \\
\text { Zusatzangaben zur Person }\end{array}$ & $\frac{\text { LQ-13 }}{\text { LEBENSQ }}$ \\
\hline \multicolumn{3}{|c|}{ Teil L (b) (Zusatzangaben zur Person) } \\
\hline \multicolumn{3}{|c|}{$\begin{array}{l}\text { 5. Welchen beruflichen Ausbildungsabschluss haben Sie? } \\
\text { (Bitte alle zutreffenden Abschlüsse ankreuzen.) }\end{array}$} \\
\hline $\begin{array}{l}\square \text { keine und nic } \\
\square \text { noch in berufl } \\
\square \text { beruflich-betr } \\
\square \text { beruflich-sch } \\
\square \text { Fach-/Meister } \\
\square \text { Fachhochsch } \\
\square \text { Hochschule } \\
\square \text { anderer Beru }\end{array}$ & $\begin{array}{l}\text { bildung } \\
\text { (Lehre) } \\
\text { Berufsfach-/Handelsschule) } \\
\text { erufs-/Fachakademie }\end{array}$ & \\
\hline
\end{tabular}

6. Sind Sie zur Zeit erwerbstätig? Was auf dieser Liste trifft auf Sie zu?

Achtung: Unter Erwerbstätigkeit wird jede bezahlte bzw. mit einem Einkommen verbundene Tätigkeit verstanden, egal welchen zeitlichen Umfang sie hat.

O Vollzeit $\geq 35 \mathrm{~h} /$ Woche

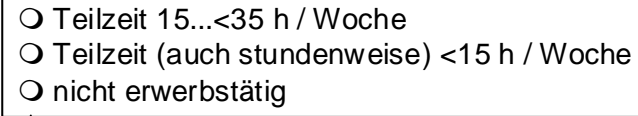

falls hier angekreuzt:

\section{Grund für Teilzeittätigkeit bzw. keine Erwerbstätigkeit}

O (bedingt) erwerbsunfähig wegen Herzinsuffizienz

$O$ (bedingt) erwerbsunfähig aus anderem Grund

O Rentner(in) / Pensionär(in) / Vorruhestand (jedoch nicht Frührente w egen Eww erbsunfähigkeit)

O arbeitslos / Null-Kurzarbeit / Umschulung

O Hausfrau / Hausmann

O Mutterschafts- / Erziehungsurlaub oder sonst. Beurlaubung

O Schüler/in

O Student/in

$O$ anderer Grund:

7. Wie hoch ist das monatliche Nettoeinkommen Ihres Haushalts insge samt?

Gemeint ist dabei die Summe, die sich aus Lohn, Gehalt, Einkommen aus selbständiger Tätigkeit, Rente oder Pension jeweils nach Abzug der Steuern und Sozialversicherungsbeiträge ergibt. Rechnen Sie bitte auch die Einkünfte aus öffentlichen Beihilfen, Einkommen aus Vermietung, Verpachtung, Wohngeld, Kindergeld und sonstige Einkünfte hinzu; bei Selbstständigen durchschnittliches Nettoeinkommen, abzüglich der Betriebsausgaben.

\begin{tabular}{|c|c|c|c|}
\hline O & Unter $500 €$ & $\mathrm{O}$ & 2000 bis unter $2500 €$ \\
\hline & 500 bis unter $1000 €$ & O & 2500 bis unter 300 \\
\hline & 1000 bis unter $1500 €$ & 0 & 3000 bis unter 3500 \\
\hline & 1500 bis unter $2000 €$ & 0 & $3500 €$ und mehr \\
\hline
\end{tabular}

8. Wie sind Sie krankenversichert?
O Gesetzliche Krankenkasse
O Private Krankenkasse
O Sozialamt

Bitte überprüfen Sie noch einmal, ob Sie alle Fragen beantwortet haben! Vielen Dank für Ihre Mitarbeit! 


\subsection{Parameter HRV}

\subsubsection{Time Domain}

Ein Vergleich von Time-Domain-Parametern zwischen Messungen von mehreren Stunden und einigen Minuten ist nicht sinnvoll. Dies gilt insbesondere für die globalen HRV-Indices wie SDNN (TASK FORCE OF THE EUROPEAN SOCIETY OF CARDIOLOGY AND THE NORTH AMERICAN SOCIETY OF PACING AND ELECTROPHYSIOLOGY 1996).

\begin{tabular}{|c|c|c|c|}
\hline $\begin{array}{c}\text { Time Domain } \\
\text { Parameter }\end{array}$ & Definition & $\begin{array}{c}\text { Einheit; } \\
\text { Berechnung }\end{array}$ & Bedeutung \\
\hline Min & minimale HR & $\begin{array}{c}\text { Bpm; Von N } \\
\text { zu N berech- } \\
\text { net }\end{array}$ & \\
\hline Med & mittlere HR & Bpm; s. „Min“ & \\
\hline Max & maximale HR & Bpm; s. „Min“ & \\
\hline QRS & $\begin{array}{l}\text { Gesamtanzahl } \\
\text { QRS-Komplexe }\end{array}$ & & \\
\hline SDNN & $\begin{array}{c}\text { SD aller gewähl- } \\
\text { ten RR-Intervalle } \\
\text { (RR-Intervalle } \\
\text { entspricht NN- } \\
\text { Intervalle: Nor- } \\
\text { malschlag zu } \\
\text { Normalschlag) }\end{array}$ & $\begin{array}{l}\quad \text { ms; } \\
\text { über ein be- } \\
\text { stimmtes Zeit- } \\
\text { intervall hin- } \\
\text { weg berech- } \\
\text { net, das ange- } \\
\text { geben werden } \\
\text { muss }\end{array}$ & $\begin{array}{l}\text { Globaler Marker der HRV; für } \\
\text { kurze Messungen geeignet. } \\
\text { Messungen über Minuten sind } \\
\text { nicht mit Messungen über } \\
\text { Stunden vergleichbar. Je län- } \\
\text { ger, umso höher. } \\
\text { Wenn die Grundfrequenz } \\
\text { schwankt, schwankt SDNN } \\
\text { auch. SDNN korreliert mit Total } \\
\text { Power. }\end{array}$ \\
\hline rMSSD & $\begin{array}{l}\text { Standardabwei- } \\
\text { chung des Mit- } \\
\text { tels der quadrier- } \\
\text { ten Differenzen } \\
\text { benachbarter } \\
\text { RR-Intervalle }\end{array}$ & $\mathrm{ms}$ & $\begin{array}{l}\text { Veränderung von Schlag zu } \\
\text { Schlag; Einschätzung kurzfristi- } \\
\text { ger Komponenten der HRV } \\
\text { hiermit möglich. RMSSD korre- } \\
\text { liert mit HF und pNN50. Stabile- } \\
\text { rer Parameter als pNN50. } \\
\text { Vagaler Indikator. }\end{array}$ \\
\hline pNN50 & $\begin{array}{l}\text { Prozent der NN- } \\
\text { Intervalldifferen- } \\
\text { zen, die } 50 \mathrm{~ms} \\
\text { überschreiten }\end{array}$ & $\%$ & $\begin{array}{c}\text { Korreliert mit HF und rMSSD. } \\
\text { Vagaler Indikator }\end{array}$ \\
\hline Gültig & $\begin{array}{l}\text { Prozentualer } \\
\text { Anteil der für die } \\
\text { HRV-Analyse } \\
\text { verwendeten } \\
\text { Intervalle }\end{array}$ & $\%$ & \\
\hline
\end{tabular}

Tabelle 18 Time Domain Parameter (MEDILOG DARWIN HOLTER ANALYSIS 2006a ; TASK FORCE OF THE EUROPEAN SOCIETY OF CARDIOLOGY AND THE NORTH AMERICAN SOCIETY OF PACING AND ELECTROPHYSIOLOGY 1996) 


\subsubsection{Frequency Domain}

Es werden Frequenzen unter $40 \mathrm{~Hz}$ erwartet (MEDILOG DARWIN HOLTER ANALYSIS 2004). Aus den EKG-Rohdaten werden mit parametrischen (Autoregression) oder nichtparametrischen (Fast-Fourrier-Transformation) Methoden die „power spectral density” berechnet (YERAGANI 1995; TASK FORCE OF THE EUROPEAN SOCIETY OF CARDIOLOGY AND THE NORTH AMERICAN SOCIETY OF PACING AND ELECTROPHYSIOLOGY 1996). Die Methoden liefern ähnliche Ergebnisse (TASK FORCE OF THE EUROPEAN SOCIETY OF CARDIOLOGY AND THE NORTH AMERICAN SOCIETY OF PACING AND ELECTROPHYSIOLOGY 1996). Die Berechnungsverfahren können systematische Änderungen der Grundfrequenz nicht bereinigen. Um dies zu eliminieren ist lineares Detrending sinnvoll (YERAGANI 1995). Die Parameter können entweder als Absolutwerte der Power (Amplitude der Sinuswelle) oder als relativer Anteil (normalisierter Anteil= „,normalizes units“) an der Gesamtpower (abzüglich der VLF-Komponente) angegeben werden. Für Kurzzeitmessungen (2-5 Minuten) sind die Frequency-Domain-Parameter gut geeignet. Für eine sinnvolle Bestimmung der HF braucht man mindestens eine Minute EKG, für die LF zwei Minuten. Fünf-Minuten-Messungen sind im Sinne der Standardisierung zu bevorzugen, falls der Versuchsaufbau dies zulässt (TASK FORCE OF THE EUROPEAN SOCIETY OF CARDIOLOGY AND THE NORTH AMERICAN SOCIETY OF PACING AND ELECTROPHYSIOLOGY 1996). 


\begin{tabular}{|c|c|c|c|}
\hline $\begin{array}{l}\text { Frequency } \\
\text { Domain } \\
\text { Parameter }\end{array}$ & Definition & $\begin{array}{c}\text { Einheit; } \\
\text { Berechnung }\end{array}$ & Bedeutung \\
\hline LF & $\begin{array}{l}\text { Spektralleistung im } \\
\text { Frequenzband } \\
0,04-0,15 \mathrm{~Hz}\end{array}$ & $\begin{array}{c}\text { „absolute } \\
\text { power" }\left(\mathrm{ms}^{2}\right) \\
\text { Short-term }(2- \\
5 \mathrm{~min})\end{array}$ & $\begin{array}{c}\text { Kombination sympathischer } \\
\text { Oszillationen (Mayer-Wellen) } \\
\text { und parasympathischer Reak- } \\
\text { tion (Baroreflex) darauf }\end{array}$ \\
\hline HF & $\begin{array}{l}\text { Spektralleistung im } \\
\text { Frequenzband } \\
0,15-0,40 \mathrm{~Hz}\end{array}$ & $\begin{array}{c}\text { „absolute } \\
\text { power" }\left(\mathrm{ms}^{2}\right) \\
\text { Short-term }(2- \\
5 \mathrm{~min})\end{array}$ & $\begin{array}{l}\text { Efferente Vagusaktivität; at- } \\
\text { mungsabhängig; gutes Maß } \\
\text { für die RSA; } \\
\text { Korreliert mit rMSSD, } \\
\text { vagaler Indikator. }\end{array}$ \\
\hline Total & $\begin{array}{l}\text { Spektralleistung im } \\
\text { Frequenzband } \\
0,0-0,4 \mathrm{~Hz}\end{array}$ & $\mathrm{~ms}^{2}$ & \\
\hline $\begin{array}{l}\text { Log LF/HF } \\
\text { (= Ln LF/HF) }\end{array}$ & $\begin{array}{l}\text { Logarithmus natu- } \\
\text { ralis des Verhält- } \\
\text { nisses zwischen } \\
\text { den Spektralleis- } \\
\text { tungen des LF- } \\
\text { und des HF- } \\
\text { Bandes }\end{array}$ & & $\begin{array}{c}\text { sympatho-vagale Balance; } \\
\text { Modulationen der HR durch } \\
\text { den Sympathikus }\end{array}$ \\
\hline
\end{tabular}

Tabelle 19 Frequency Domain (TASK FORCE OF THE EUROPEAN SOCIETY OF CARDIOLOGY AND THE NORTH AMERICAN SOCIETY OF PACING AND ELECTROPHYSIOLOGY 1996; MEDILOG DARWIN HOLTER ANALYSIS 2006a; FRENNEAUX 2004, MALIK 1998)

Normwerte für Time und Frequency Domain finden sich z.B. in TASK FORCE OF THE EUROPEAN SOCIETY OF CARDIOLOGY AND THE NORTH AMERICAN SOCIETY OF PACING AND ELECTROPHYSIOLOGY 1996 oder AGELINK et al. 2001.

\subsection{HRV nach Alter und Geschlecht}

Das hier untersuchte Kollektiv zeigte sowohl zwischen den Altersgruppen als auch zwischen den Geschlechtern signifikante Mittelwertsunterschiede. Im Folgenden befindet sich eine Aufstellung der gemessenen Werte nach Alter, nach Geschlecht und nach Alter und Geschlecht aufgeschlüsselt. In den HRV-Tabellen werden Mittelwertsunterschiede durch Kennzeichnung mit Sternen je nach Signifikanzniveau markiert $(*=\mathrm{p}<0,05 ; \quad * *=\mathrm{p}<0,01$; $* * *=\mathrm{p}<0,001)$. In den übrigen Tabellen sind nur signigikante Ergebnisse aufgeführt. Die Signifikanzprüfung wurde mit T-Testungen mit getrennten Varianzen zwischen Gruppen jeweils für die drei Phasen durchgeführt. Dabei waren die HR und die HRV-Parameter die abhängigen Parameter und das Geschlecht und die Altersdekaden die Gruppierungsvariablen. Zur Übersicht zunächst eine Darstellung der Altersverteilung im Kollektiv nach Dekaden. 


\section{Gesamt Prozent Männer Prozent Frauen Prozent}

\begin{tabular}{ccccccc}
\hline $\mathbf{5 0 - 6 0}$ & 103 & 22,01 & 53 & 24,09 & 50 & 20,16 \\
\hline $\mathbf{6 1 - 7 0}$ & 225 & 48,08 & 106 & 48,18 & 119 & 47,98 \\
\hline $\mathbf{7 1 - 8 0}$ & 118 & 25,21 & 54 & 24,55 & 64 & 25,81 \\
\hline$>\mathbf{8 0}$ & 22 & 4,70 & 7 & 3,18 & 15 & 6,05 \\
\hline Missing & 0 & 0 & 0 & 0 & 0 & 0 \\
\hline Gesamt & 468 & 100 & 220 & 100 & 248 & 100 \\
\hline
\end{tabular}

Tabelle 20 Altersdekaden mit dazugehörigen Probandenzahlen

Die Mittelwerte der HR- und HRV-Parameter nach Geschlecht und Altersdekade sind im Weiteren aufgeführt.

\begin{tabular}{|c|c|c|c|c|c|c|c|c|c|}
\hline \multirow{2}{*}{$\begin{array}{l}\text { Gesamt- } \\
\text { kollektiv }\end{array}$} & \multicolumn{3}{|c|}{ Alle } & \multicolumn{3}{|c|}{ Männer } & \multicolumn{3}{|c|}{ Frauen } \\
\hline & $\mathbf{N}$ & Mittelwert & SD & $\mathbf{N}$ & Mittelwert & SD & $\mathbf{N}$ & Mittelwert & SD \\
\hline \multicolumn{10}{|c|}{ Gehphase ( 2 Minuten) } \\
\hline $\begin{array}{c}\text { mittlere } \\
\text { HR }\end{array}$ & 423 & 104,51 & 17,53 & 205 & 103,88 & 17,03 & 218 & 105,10 & 18,02 \\
\hline $\begin{array}{c}\text { Ln } \\
\text { SDNN }\end{array}$ & 423 & 2,82 & 0,55 & 205 & 2,78 & 0,56 & 218 & 2,86 & 0,55 \\
\hline $\begin{array}{c}\text { Ln } \\
\text { rMSSD* }\end{array}$ & 423 & 2,78 & 0,83 & 205 & 2,67 & 0,88 & 218 & 2,87 & 0,76 \\
\hline pNN50 & 421 & 0,03 & 0,059 & 204 & 0,03 & 0,06 & 217 & 0,04 & 0,060 \\
\hline Ln LF & 423 & 2,31 & 1,36 & 205 & 2,28 & 1,37 & 218 & 2,33 & 1,36 \\
\hline Ln HF & 423 & 2,23 & 1,62 & 205 & 2,08 & 1,66 & 218 & 2,37 & 1,56 \\
\hline Ln Total & 423 & 3,91 & 1,20 & 205 & 3,89 & 1,21 & 218 & 3,94 & 1,18 \\
\hline $\begin{array}{c}\text { Ln } \\
\text { LF/HF* }\end{array}$ & 423 & 0,03 & 0,48 & 205 & 0,09 & 0,49 & 218 & $-0,015$ & 0,46 \\
\hline \multicolumn{10}{|c|}{ Taktatmungsphase (5 Minuten) } \\
\hline $\begin{array}{c}\text { mittlere } \\
\text { HR }\end{array}$ & 444 & 73,56 & 12,69 & 213 & 73,17 & 12,33 & 231 & 73,93 & 13,04 \\
\hline $\begin{array}{c}\text { Ln } \\
\text { SDNN }\end{array}$ & 442 & 3,53 & 0,52 & 212 & 3,49 & 0,53 & 230 & 3,58 & 0,50 \\
\hline $\begin{array}{c}\text { Ln } \\
\text { rMSSD }\end{array}$ & 444 & 3,10 & 0,79 & 213 & 3,03 & 0,78 & 231 & 3,16 & 0,79 \\
\hline pNN50 & 44 & 0,052 & 0,076 & 212 & 0,048 & 0,074 & 229 & 0,056 & 78 \\
\hline Ln LF & 443 & 5,20 & 1,13 & 213 & 5,14 & 1,18 & 230 & 5,26 & 08 \\
\hline
\end{tabular}




\begin{tabular}{cccccccccc}
\hline Ln HF* & 444 & 3,87 & 1,39 & 213 & 3,73 & 1,37 & 231 & 4,00 & 1,40 \\
\hline Ln Total $^{*}$ & 437 & 6,65 & 0,99 & 208 & 6,54 & 1,063 & 229 & 6,75 & 0,92 \\
\hline Ln LF/HF & 443 & 0,58 & 0,46 & 213 & 0,61 & 0,46 & 230 & 0,56 & 0,46 \\
\hline
\end{tabular}

\section{Ruhephase ( 5 Minuten)}

\begin{tabular}{cccccccccc}
\hline $\begin{array}{c}\text { mittlere } \\
\text { HR }\end{array}$ & 431 & 72,49 & 12,29 & 205 & 72,04 & 12,24 & 226 & 72,89 & 12,35 \\
\hline Ln SDNN & 429 & 3,39 & 0,47 & 205 & 3,39 & 0,46 & 224 & 3,38 & 0,47 \\
\hline Ln rMSSD & 431 & 2,88 & 0,80 & 205 & 2,90 & 0,78 & 226 & 2,86 & 0,83 \\
\hline pNN50 & 431 & 0,029 & 0,053 & 205 & 0,026 & 0,043 & 226 & 0,032 & 0,061 \\
\hline Ln LF & 431 & 4,85 & 1,22 & 205 & 4,78 & 1,18 & 226 & 4,92 & 1,26 \\
\hline Ln HF* & 431 & 3,39 & 1,34 & 205 & 3,24 & 1,25 & 226 & 3,52 & 1,40 \\
\hline Ln Total & 430 & 6,01 & 0,99 & 205 & 5,94 & 0,94 & 225 & 6,08 & 1,02 \\
\hline Ln LF/HF & 430 & 0,64 & 0,44 & 204 & 0,67 & 0,46 & 226 & 0,61 & 0,41 \\
\hline
\end{tabular}

Tabelle 21 Gesamtkollektiv aufgetrennt nach Geschlecht. An der HR und den HRV-Parametern wurde ein evtl. signifikanter Mittelwertsunterschied bei einer Testung Männer gegen Frauen mit Sternen markiert.

\begin{tabular}{|c|c|c|c|c|c|c|c|c|c|}
\hline \multirow{2}{*}{$50-60$} & \multicolumn{3}{|c|}{ Alle } & \multicolumn{3}{|c|}{ Männer } & \multicolumn{3}{|c|}{ Frauen } \\
\hline & $\mathbf{N}$ & Mittelwert & SD & $\mathbf{N}$ & Mittelwert & SD & $\mathbf{N}$ & Mittelwert & SD \\
\hline \multicolumn{10}{|c|}{ Gehphase ( 2 Minuten) } \\
\hline $\begin{array}{c}\text { mittlere } \\
\text { HR }\end{array}$ & 98 & 110,51 & 20,70 & 53 & 110,21 & 17,71 & 45 & 110,87 & 23,95 \\
\hline $\begin{array}{c}\text { Ln } \\
\text { SDNN }\end{array}$ & 98 & 2,75 & 0,55 & 53 & 2,78 & 0,57 & 45 & 2,71 & 0,53 \\
\hline $\begin{array}{c}\text { Ln } \\
\text { rMSSD }\end{array}$ & 98 & 2,55 & 0,83 & 53 & 2,40 & 0,95 & 45 & 2,73 & 0,61 \\
\hline pNN50 & 98 & 0,02 & 0,03 & 53 & 0,02 & 0,03 & 45 & 0,02 & 0,04 \\
\hline Ln LF & 98 & 2,15 & 1,28 & 53 & 2,17 & 1,24 & 45 & 2,11 & 1,33 \\
\hline Ln HF & 98 & 1,70 & 1,49 & 53 & 1,49 & 1,58 & 45 & 1,95 & 1,35 \\
\hline $\begin{array}{c}\text { Ln To- } \\
\text { tal }\end{array}$ & 98 & 3,71 & 1,13 & 53 & 3,76 & 1,11 & 45 & 3,64 & 1,16 \\
\hline $\begin{array}{c}\text { Ln } \\
\text { LF/HF }\end{array}$ & 98 & 0,19 & 0,48 & 53 & 0,29 & 0,51 & 45 & 0,07 & 0,40 \\
\hline
\end{tabular}




\begin{tabular}{cccccccccc}
\multicolumn{2}{l}{ Taktatmungsphase (5 Minuten) } \\
\begin{tabular}{cccccccccc}
\multicolumn{2}{c}{ mittlere } \\
HR
\end{tabular} & 101 & 76,35 & 13,40 & 53 & 76,07 & 12,17 & 48 & 76,65 & 14,76 \\
\hline Ln SDNN & 101 & 3,63 & 0,50 & 53 & 3,60 & 0,52 & 48 & 3,65 & 0,48 \\
\hline Ln rMSSD & 101 & 3,02 & 0,69 & 53 & 3,01 & 0,67 & 48 & 3,03 & 0,73 \\
\hline pNN50 & 101 & 0,05 & 0,06 & 53 & 0,05 & 0,06 & 48 & 0,05 & 0,07 \\
\hline Ln LF & 101 & 5,56 & 1,11 & 53 & 5,59 & 1,16 & 48 & 5,52 & 1,08 \\
\hline Ln HF & 101 & 3,77 & 1,29 & 53 & 3,75 & 1,26 & 48 & 3,80 & 1,34 \\
\hline Ln Total & 101 & 6,85 & 0,99 & 53 & 6,89 & 1,01 & 48 & 6,81 & 0,97 \\
\hline Ln LF/HF & 101 & 0,78 & 0,37 & 53 & 0,80 & 0,39 & 48 & 0,75 & 0,33 \\
\hline
\end{tabular}

Ruhephase ( 5 Minuten)

\begin{tabular}{cccccccccc}
\hline $\begin{array}{c}\text { mittlere } \\
\text { HR }\end{array}$ & 101 & 75,33 & 13,42 & 53 & 75,02 & 12,51 & 48 & 75,67 & 14,48 \\
\hline Ln SDNN & 101 & 3,42 & 0,46 & 53 & 3,47 & 0,44 & 48 & 3,37 & 0,47 \\
\hline Ln rMSSD & 101 & 2,73 & 0,76 & 53 & 2,76 & 0,77 & 48 & 2,69 & 0,75 \\
\hline pNN50 & 101 & 0,03 & 0,05 & 53 & 0,02 & 0,04 & 48 & 0,03 & 0,05 \\
\hline Ln LF & 101 & 5,35 & 1,05 & 53 & 5,34 & 1,06 & 48 & 5,36 & 1,06 \\
\hline Ln HF & 101 & 3,34 & 1,27 & 53 & 3,23 & 1,25 & 48 & 3,45 & 1,30 \\
\hline Ln Total & 101 & 6,27 & 0,89 & 53 & 6,24 & 0,84 & 48 & 6,30 & 0,94 \\
\hline Ln LF/HF & 101 & 0,87 & 0,36 & 53 & 0,91 & 0,40 & 48 & 0,83 & 0,30 \\
\hline
\end{tabular}

Tabelle 22 HR und HRV-Parameter der Altersdekade 50-60 nach Phasen getrennt und nach Geschlecht aufgeteilt. 


\begin{tabular}{|c|c|c|c|c|c|c|c|c|c|}
\hline \multirow{2}{*}{$61-70$} & \multicolumn{3}{|c|}{ Alle } & \multicolumn{3}{|c|}{ Männer } & \multicolumn{3}{|c|}{ Frauen } \\
\hline & $\mathbf{N}$ & Mittelwert & SD & $\mathbf{N}$ & Mittelwert & SD & $\mathbf{N}$ & Mittelwert & SD \\
\hline \multicolumn{10}{|c|}{ Gehphase ( 2 Minuten) } \\
\hline $\begin{array}{c}\text { mittlere } \\
\text { HR }\end{array}$ & 203 & 104,58 & 16,19 & 100 & 103,50 & 16,44 & 103 & 105,62 & 15,96 \\
\hline $\begin{array}{c}\text { Ln } \\
\text { SDNN }\end{array}$ & 203 & 2,77 & 0,53 & 100 & 2,70 & 0,52 & 103 & 2,83 & 0,54 \\
\hline $\begin{array}{c}\text { Ln } \\
\text { rMSSD }\end{array}$ & 203 & 2,75 & 0,80 & 100 & 2,68 & 0,78 & 103 & 2,82 & 0,81 \\
\hline pNN50 & 201 & 0,03 & 0,04 & 99 & 0,02 & 0,03 & 102 & 0,03 & 0,05 \\
\hline Ln LF & 203 & 2,26 & 1,37 & 100 & 2,20 & 1,35 & 103 & 2,31 & 1,39 \\
\hline Ln HF & 203 & 2,19 & 1,59 & 100 & 2,07 & 1,54 & 103 & 2,31 & 1,64 \\
\hline $\begin{array}{c}\text { Ln To- } \\
\text { tal }\end{array}$ & 203 & 3,89 & 1,18 & 100 & 3,84 & 1,19 & 103 & 3,93 & 1,18 \\
\hline $\begin{array}{c}\text { Ln } \\
\text { LF/HF }\end{array}$ & 203 & 0,03 & 0,48 & 100 & 0,06 & 0,46 & 103 & $-0,003$ & 0,50 \\
\hline \multicolumn{10}{|c|}{ Taktatmungsphase (5 Minuten) } \\
\hline $\begin{array}{c}\text { mittlere } \\
\text { HR }\end{array}$ & 213 & 73,15 & 12,93 & 102 & 73,44 & 13,12 & 111 & 72,89 & 12,80 \\
\hline $\begin{array}{c}\text { Ln } \\
\text { SDNN }\end{array}$ & 211 & 3,50 & 0,52 & 101 & 3,41 & 0,50 & 110 & 3,58 & 0,52 \\
\hline $\begin{array}{c}\text { Ln } \\
\text { rMSSD }\end{array}$ & 213 & 3,06 & 0,76 & 102 & 2,94 & 0,73 & 111 & 3,16 & 0,77 \\
\hline pNN50 & 212 & 0,05 & 0,08 & 102 & 0,04 & 0,08 & 110 & 0,055 & 0,078 \\
\hline Ln LF & 212 & 5,18 & 1,11 & 102 & 5,03 & 1,18 & 110 & 5,32 & 1,03 \\
\hline Ln HF & 213 & 3,79 & 1,34 & 102 & 3,50 & 1,31 & 111 & 4,05 & 1,32 \\
\hline $\begin{array}{c}\text { Ln To- } \\
\text { tal }\end{array}$ & 208 & 6,63 & 1,00 & 99 & 6,41 & 1,07 & 109 & 6,82 & 0,90 \\
\hline $\begin{array}{c}\text { Ln } \\
\text { LF/HF }\end{array}$ & 212 & 0,61 & 0,43 & 102 & 0,66 & 0,42 & 110 & 0,56 & 0,43 \\
\hline \multicolumn{10}{|c|}{ Ruhephase (5 Minuten) } \\
\hline $\begin{array}{c}\text { mittlere } \\
\text { HR }\end{array}$ & 211 & 71,88 & 12,29 & 99 & 72,03 & 12,72 & 112 & 71,76 & 11,94 \\
\hline $\begin{array}{c}\text { Ln } \\
\text { SDNN }\end{array}$ & 209 & 3,37 & 0,47 & 99 & 3,36 & 0,45 & 110 & 3,37 & 0,48 \\
\hline $\begin{array}{c}\text { Ln } \\
\text { rMSSD }\end{array}$ & 211 & 2,84 & 0,78 & 99 & 2,84 & 0,69 & 112 & 2,84 & 0,85 \\
\hline pNN50 & 211 & 0,03 & 0,06 & 99 & 0,02 & 0,04 & 112 & 0,04 & 0,073 \\
\hline Ln LF & 211 & 4,84 & 1,23 & 99 & 4,71 & 1,16 & 112 & 4,95 & 1,29 \\
\hline
\end{tabular}




\begin{tabular}{cccccccccc}
\hline Ln HF & 211 & 3,40 & 1,34 & 99 & 3,18 & 1,17 & 112 & 3,59 & 1,45 \\
\hline $\begin{array}{c}\text { Ln To- } \\
\text { tal }\end{array}$ & 210 & 6,01 & 1,01 & 99 & 5,90 & 0,93 & 111 & 6,11 & 1,07 \\
\hline $\begin{array}{c}\text { Ln } \\
\text { LF/HF }\end{array}$ & 211 & 0,63 & 0,42 & 99 & 0,66 & 0,43 & 112 & 0,59 & 0,40 \\
\hline
\end{tabular}

Tabelle 23 HR und HRV-Parameter der Altersdekade 61-70 nach Phasen getrennt und nach Geschlecht aufgeteilt.

\begin{tabular}{|c|c|c|c|c|c|c|c|c|c|}
\hline \multirow{2}{*}{$71-80$} & \multicolumn{3}{|c|}{ Alle } & \multicolumn{3}{|c|}{ Männer } & \multicolumn{3}{|c|}{ Frauen } \\
\hline & $\mathbf{N}$ & Mittelwert & SD & $\mathbf{N}$ & Mittelwert & SD & $\mathbf{N}$ & Mittelwert & SD \\
\hline \multicolumn{10}{|c|}{ Gehphase ( 2 Minuten) } \\
\hline $\begin{array}{c}\text { mittlere } \\
\text { HR }\end{array}$ & 104 & 99,38 & 15,69 & 47 & 98,18 & 15,840 & 57 & 100,38 & 15,63 \\
\hline $\begin{array}{c}\text { Ln } \\
\text { SDNN }\end{array}$ & 104 & 2,93 & 0,55 & 47 & 2,88 & 0,56 & 57 & 2,96 & 0,54 \\
\hline $\begin{array}{c}\text { Ln } \\
\text { rMSSD }\end{array}$ & 104 & 2,94 & 0,84 & 47 & 2,88 & 0,93 & 57 & 2,99 & 0,76 \\
\hline pNN50 & 104 & 0,05 & 0,090 & 47 & 0,054 & 0,097 & 57 & 0,047 & 0,085 \\
\hline Ln LF & 104 & 2,53 & 1,36 & 47 & 2,48 & 1,41 & 57 & 2,57 & 1,33 \\
\hline Ln HF & 104 & 2,62 & 1,63 & 47 & 2,59 & 1,75 & 57 & 2,65 & 1,55 \\
\hline $\begin{array}{c}\text { Ln To- } \\
\text { tal }\end{array}$ & 104 & 4,13 & 1,21 & 47 & 4,038 & 1,27 & 57 & 4,20 & 1,17 \\
\hline $\begin{array}{c}\text { Ln } \\
\text { LF/HF }\end{array}$ & 104 & $-0,04$ & 0,44 & 47 & $-0,048$ & 0,47 & 57 & $-0,035$ & 0,41 \\
\hline
\end{tabular}

Taktatmungsphase (5 Minuten)

\begin{tabular}{|c|c|c|c|c|c|c|c|c|c|}
\hline $\begin{array}{c}\text { mittlere } \\
\text { HR }\end{array}$ & 109 & 71,79 & 11,78 & 51 & 69,97 & 10,76 & 58 & 73,38 & 12,47 \\
\hline $\begin{array}{c}\text { Ln } \\
\text { SDNN }\end{array}$ & 109 & 3,50 & 0,50 & 51 & 3,51 & 0,52 & 58 & 3,50 & 0,50 \\
\hline $\begin{array}{c}\text { Ln } \\
\text { rMSSD }\end{array}$ & 109 & 3,13 & 0,86 & 51 & 3,14 & 0,88 & 58 & 3,12 & 0,85 \\
\hline pNN50 & 107 & 0,05 & 0,08 & 50 & 0,054 & 0,079 & 57 & 0,053 & 0,083 \\
\hline Ln LF & 109 & 4,99 & 1,04 & 51 & 4,95 & 1,047 & 58 & 5,02 & 1,033 \\
\hline Ln HF & 109 & 3,99 & 1,48 & 51 & 4,005 & 1,47 & 58 & 3,97 & 1,50 \\
\hline $\begin{array}{c}\text { Ln To- } \\
\text { tal }\end{array}$ & 107 & 6,56 & 0,92 & 49 & 6,47 & 0,96 & 58 & 6,64 & 0,90 \\
\hline $\begin{array}{c}\text { Ln } \\
\text { LF/HF }\end{array}$ & 109 & 0,43 & 0,50 & 51 & 0,41 & 0,45 & 58 & 0,46 & 0,55 \\
\hline
\end{tabular}

Ruhephase ( 5 Minuten)

\begin{tabular}{llllllllll}
\hline mittlere & 102 & 70,91 & 11,40 & 47 & 68,91 & 10,80 & 55 & 72,62 & 11,71
\end{tabular} HR 


\begin{tabular}{cccccccccc}
\hline $\begin{array}{c}\text { Ln } \\
\text { SDNN }\end{array}$ & 102 & 3,37 & 0,47 & 47 & 3,34 & 0,48 & 55 & 3,39 & 0,47 \\
\hline $\begin{array}{c}\text { Ln } \\
\text { rMSSD }\end{array}$ & 102 & 3,00 & 0,83 & 47 & 3,10 & 0,82 & 55 & 2,91 & 0,83 \\
\hline pNN50 & 102 & 0,028 & 0,039 & 47 & 0,032 & 0,041 & 55 & 0,023 & 0,037 \\
\hline Ln LF & 102 & 4,47 & 1,14 & 47 & 4,39 & 1,052 & 55 & 4,53 & 1,22 \\
\hline Ln HF & 102 & 3,30 & 1,33 & 47 & 3,33 & 1,33 & 55 & 3,27 & 1,35 \\
\hline $\begin{array}{c}\text { Ln To- } \\
\text { tal }\end{array}$ & 102 & 5,77 & 0,93 & 47 & 5,71 & 0,92 & 55 & 5,83 & 0,94 \\
\hline $\begin{array}{c}\text { Ln } \\
\text { LF/HF }\end{array}$ & 102 & 0,51 & 0,45 & 47 & 0,46 & 0,47 & 55 & 0,55 & 0,42 \\
\hline
\end{tabular}

Tabelle 24 HR und HRV-Parameter der Altersdekade 71-80 nach Phasen getrennt und nach Geschlecht aufgeteilt.

\begin{tabular}{|c|c|c|c|c|c|c|c|c|c|}
\hline \multirow{2}{*}{$>80$} & \multicolumn{3}{|c|}{ Alle } & \multicolumn{3}{|c|}{ Männer } & \multicolumn{3}{|c|}{ Frauen } \\
\hline & $\mathbf{N}$ & Mittelwert & SD & $\mathbf{N}$ & Mittelwert & SD & $\mathbf{N}$ & Mittelwert & SD \\
\hline \multicolumn{10}{|c|}{ Gehphase ( 2 Minuten) } \\
\hline $\begin{array}{c}\text { mittlere } \\
\text { HR }\end{array}$ & 18 & 100,69 & 14,001 & 5 & 98,01 & 13,19 & 13 & 101,72 & 14,68 \\
\hline $\begin{array}{l}\text { Ln } \\
\text { SDNN }\end{array}$ & 18 & 3,14 & 0,61 & 5 & 3,32 & 0,71 & 13 & 3,06 & 0,59 \\
\hline $\begin{array}{c}\text { Ln } \\
\text { rMSSD }\end{array}$ & 18 & 3,36 & 0,72 & 5 & 3,61 & 0,57 & 13 & 3,26 & 0,77 \\
\hline pNN50 & 18 & 0,053 & 0,048 & 5 & 0,069 & 0,054 & 13 & 0,046 & 0,046 \\
\hline Ln LF & 18 & 2,52 & 1,70 & 5 & 3,16 & 2,51 & 13 & 2,27 & 1,32 \\
\hline Ln HF & 18 & 3,21 & 1,52 & 5 & 3,81 & 1,59 & 13 & 2,98 & 1,49 \\
\hline $\begin{array}{l}\text { Ln To- } \\
\text { tal }\end{array}$ & 18 & 4,10 & 1,43 & 5 & 4,63 & 1,93 & 13 & 3,89 & 1,22 \\
\hline $\begin{array}{c}\text { Ln } \\
\text { LF/HF }\end{array}$ & 18 & $-0,30$ & 0,52 & 5 & $-0,28$ & 0,53 & 13 & $-0,30$ & 0,54 \\
\hline \multicolumn{10}{|c|}{ Taktatmungsphase (5 Minuten) } \\
\hline $\begin{array}{c}\text { mittlere } \\
\text { HR }\end{array}$ & 21 & 73,59 & 9,68 & 7 & 70,52 & 7,56 & 14 & 75,13 & 10,50 \\
\hline $\begin{array}{c}\text { Ln } \\
\text { SDNN }\end{array}$ & 21 & 3,59 & 0,63 & 7 & 3,57 & 0,88 & 14 & 3,60 & 0,49 \\
\hline $\begin{array}{c}\text { Ln } \\
\text { rMSSD }\end{array}$ & 21 & 3,75 & 0,85 & 7 & 3,71 & 1,24 & 14 & 3,77 & 0,62 \\
\hline pNN50 & 21 & 0,09 & 0,096 & 7 & 0,085 & 0,11 & 14 & 0,087 & 0,093 \\
\hline Ln LF & 21 & 4,83 & 1,45 & 7 & 4,64 & 1,59 & 14 & 4,93 & 1,43 \\
\hline Ln HF & 21 & 4,48 & 1,69 & 7 & 4,84 & 1,67 & 14 & 4,30 & 1,73 \\
\hline
\end{tabular}




\begin{tabular}{cccccccccc}
\hline $\begin{array}{c}\text { Ln To- } \\
\text { tal }\end{array}$ & 21 & 6,36 & 1,19 & 7 & 6,28 & 1,64 & 14 & 6,41 & 0,96 \\
\hline $\begin{array}{c}\text { Ln } \\
\text { LF/HF }\end{array}$ & 21 & 0,16 & 0,51 & 7 & $-0,087$ & 0,53 & 14 & 0,28 & 0,47 \\
\hline
\end{tabular}

Ruhephase ( 5 Minuten)

\begin{tabular}{cccccccccc}
\hline $\begin{array}{c}\text { mittlere } \\
\text { HR }\end{array}$ & 17 & 72,50 & 7,87 & 6 & 70,47 & 7,33 & 11 & 73,61 & 8,27 \\
\hline $\begin{array}{c}\text { Ln } \\
\text { SDNN }\end{array}$ & 17 & 3,54 & 0,54 & 6 & 3,61 & 0,75 & 11 & 3,50 & 0,43 \\
\hline $\begin{array}{c}\text { Ln } \\
\text { rMSSD }\end{array}$ & 17 & 3,42 & 0,97 & 6 & 3,43 & 1,37 & 11 & 3,42 & 0,75 \\
\hline pNN50 & 17 & 0,047 & 0,045 & 6 & 0,043 & 0,039 & 11 & 0,049 & 0,050 \\
\hline Ln LF & 17 & 4,41 & 1,51 & 6 & 3,90 & 1,67 & 11 & 4,69 & 1,42 \\
\hline Ln HF & 17 & 4,06 & 1,62 & 6 & 3,55 & 2,00 & 11 & 4,33 & 1,39 \\
\hline Ln Total & 17 & 5,88 & 1,27 & 6 & 5,67 & 1,67 & 11 & 6,00 & 1,082 \\
\hline $\begin{array}{c}\text { Ln } \\
\text { LF/HF }\end{array}$ & 16 & 0,18 & 0,42 & 5 & 0,25 & 0,44 & 11 & 0,16 & 0,42 \\
\hline
\end{tabular}

Tabelle 25 HR und HRV-Parameter der Altersdekade $>80$ nach Phasen getrennt und nach Geschlecht aufgeteilt.

Im Rahmen einer T-Testung der HR und der HRV-Parameter mit getrennten Varianzen nach Dekaden wurden die in den folgenden Tabelle aufgeführten signfikanten Auffälligkeiten gefunden.

\begin{tabular}{|c|c|c|c|c|c|c|c|c|c|c|c|c|c|c|c|c|c|c|}
\hline \multirow{2}{*}{$\begin{array}{c}\text { T-Test } \\
\text { Alle }\end{array}$} & \multicolumn{3}{|c|}{$\begin{array}{c}\text { 50-60 vs. } \\
61-70\end{array}$} & \multicolumn{3}{|c|}{$\begin{array}{c}50-60 \text { vs. } \\
71-80\end{array}$} & \multicolumn{3}{|c|}{$\begin{array}{c}50-60 \text { vs. } \\
>80\end{array}$} & \multicolumn{3}{|c|}{$\begin{array}{c}\text { 61-70 vs. } \\
71-80\end{array}$} & \multicolumn{3}{|c|}{$\begin{array}{c}\text { 61-70 vs. } \\
>80\end{array}$} & \multicolumn{3}{|c|}{$\begin{array}{c}71-80 \\
\text { vs. }>80\end{array}$} \\
\hline & $\mathbf{G}$ & $\mathbf{T}$ & $\mathbf{R}$ & $\mathbf{G}$ & $\mathbf{T}$ & $\mathbf{R}$ & $\mathbf{G}$ & $\mathbf{T}$ & $\mathbf{R}$ & $\mathbf{G}$ & $\mathbf{T}$ & $\mathbf{R}$ & $\mathbf{G}$ & $\mathbf{T}$ & $\mathbf{R}$ & $\mathbf{G}$ & $\mathbf{T}$ & $\mathbf{R}$ \\
\hline $\begin{array}{c}\text { mittlere } \\
\text { HR }\end{array}$ & * & * & * & $* * *$ & $* *$ & * & * & & & ** & & & & & & & & \\
\hline SDNN & & * & & * & & & * & & & * & & & * & & & & & \\
\hline rMSSD & * & & & $\star \star$ & & * & $* * *$ & $\star \star$ & * & & & & $* *$ & $* *$ & * & * & $\star *$ & \\
\hline pNN50 & & & & $\star \star$ & & & $\star \star$ & & & * & & & * & & & & & \\
\hline LF & & $\star *$ & $* \star *$ & * & $* * *$ & $\star \star \star *$ & & * & * & & & * & & & & & & \\
\hline HF & $* *$ & & & $* * *$ & & & $* * *$ & & & * & & & * & & & & & \\
\hline Total & & & * & * & * & $\star * \star$ & & & & & & * & & & & & & \\
\hline LF/HF & $* *$ & $* \star \star$ & $* \star \star$ & 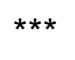 & $\star * \star$ & $\star \star \star ~$ & $\star \star$ & $* \star \star$ & 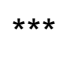 & & $\star *$ & * & * & $\star \star \star$ & $\star * \star *$ & & * & $\star *$ \\
\hline
\end{tabular}

Tabelle 26 T-Testung mit getrennten Varianzen Altersdekaden nach Untersuchungsphasen getrennt für HR und HRV-Parameter. Die Sterne geben das Signifkanzniveau an. 


\begin{tabular}{|c|c|c|c|c|c|c|c|c|c|c|c|c|c|c|c|c|c|c|}
\hline \multirow{2}{*}{$\begin{array}{l}\text { T-Test } \\
\text { Männer }\end{array}$} & \multicolumn{3}{|c|}{$\begin{array}{c}50-60 \text { vs. } \\
61-70\end{array}$} & \multicolumn{3}{|c|}{$\begin{array}{c}50-60 \text { vs. } 71- \\
80\end{array}$} & \multicolumn{3}{|c|}{$\begin{array}{c}50-60 \text { vs. } \\
>80\end{array}$} & \multicolumn{3}{|c|}{$\begin{array}{c}61-70 \text { vs. } \\
71-80\end{array}$} & \multicolumn{3}{|c|}{$\begin{array}{c}\text { 61-70 vs. } \\
>80\end{array}$} & \multicolumn{3}{|c|}{$\begin{array}{c}\text { 71-80 vS. } \\
>80\end{array}$} \\
\hline & $\mathbf{G}$ & $\mathbf{T}$ & $\mathbf{R}$ & $\mathbf{G}$ & $\mathbf{T}$ & $\mathbf{R}$ & $\mathbf{G}$ & $\mathbf{T}$ & $\mathbf{R}$ & $\mathbf{G}$ & $\mathbf{T}$ & $\mathbf{R}$ & $\mathbf{G}$ & $\mathbf{T}$ & $\mathbf{R}$ & $\mathbf{G}$ & $\mathbf{T}$ & $\mathbf{R}$ \\
\hline $\begin{array}{c}\text { mittlere } \\
\text { HR }\end{array}$ & * & & & $\star \star \star *$ & $\star *$ & * & & & & & & & & & & & & \\
\hline SDNN & & * & & & & & & & & & & & & & & & & \\
\hline rMSSD & & & & * & & * & * & & & & & & * & & & * & & \\
\hline pNN50 & & & & * & & & & & & * & & & & & & & & \\
\hline LF & & ** & $\star *$ & & ** & $\star * *$ & & & & & & & & & & & & \\
\hline HF & * & & & $* *$ & & & * & & & & * & & & & & & & \\
\hline Total & & ** & * & & * & ** & & & & & & & & & & & & \\
\hline LF/HF & ** & * & $\star \star \star$ & $\star \star \star$ & $* \star *$ & $\star \star \star$ & & ** & * & & $\star *$ & * & & $\star *$ & & & * & \\
\hline
\end{tabular}

Tabelle 27 T-Testung mit getrennten Varianzen nach Altersdekaden nur Männer nach Untersuchungsphasen getrennt für HR und HRV-Parameter. Die Sterne geben das Signifikanzniveau an.

\begin{tabular}{|c|c|c|c|c|c|c|c|c|c|c|c|c|c|c|c|c|c|c|}
\hline \multirow{2}{*}{$\begin{array}{l}\text { T-Test } \\
\text { Frauen }\end{array}$} & \multicolumn{3}{|c|}{$\begin{array}{c}50-60 \text { vs. } \\
61-70\end{array}$} & \multicolumn{3}{|c|}{$\begin{array}{c}50-60 \text { vs. } \\
71-80\end{array}$} & \multicolumn{3}{|c|}{$\begin{array}{c}50-60 \text { vs. } \\
>80\end{array}$} & \multicolumn{3}{|c|}{$\begin{array}{c}\text { 61-70 vs. } \\
71-80\end{array}$} & \multicolumn{3}{|c|}{$\begin{array}{c}\text { 61-70 vs. } \\
>80\end{array}$} & \multicolumn{3}{|c|}{$\begin{array}{c}71-80 \text { vs. } \\
>80\end{array}$} \\
\hline & $\mathbf{G}$ & $\mathbf{T}$ & $\mathbf{R}$ & $\mathbf{G}$ & $\mathbf{T}$ & $\mathbf{R}$ & $\mathbf{G}$ & $\mathbf{T}$ & $\mathbf{R}$ & $\mathbf{G}$ & $\mathbf{T}$ & $\mathbf{R}$ & $\mathbf{G}$ & $\mathbf{T}$ & $\mathbf{R}$ & $\mathbf{G}$ & $\mathbf{T}$ & $\mathbf{R}$ \\
\hline $\begin{array}{c}\text { mittlere } \\
\text { HR }\end{array}$ & & & & * & & & & & & * & & & & & & & & \\
\hline SDNN & & & & * & & & & & & & & & & & & & & \\
\hline rMSSD & & & & & & & $*$ & $* * *$ & $*$ & & & & & $* *$ & * & & $* *$ & \\
\hline \multicolumn{19}{|l|}{ pNN50 } \\
\hline LF & & & & & * & $* * *$ & & & & & & * & & & & & & \\
\hline HF & & & & * & & & * & & & & & & & & & & & * \\
\hline Total & & & & * & & * & & & & & & & & & & & & \\
\hline LF/HF & & ** & 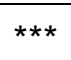 & & ** & $\star \star \star *$ & * & $\star \star$ & $\star \star \star *$ & & & & & * & ** & & & * \\
\hline
\end{tabular}

Tabelle 28 T-Testung mit getrennten Varianzen nach Altersdekaden nur Frauen nach Untersuchungsphasen getrennt für HR und HRV-Parameter. Die Sterne geben das Signifikanzniveau an. 


\subsection{HRV nach psychologischen Variablen}

\subsubsection{Depressivität}

\begin{tabular}{ccccccc}
\hline \multirow{2}{*}{ Depressivität } & \multicolumn{3}{c}{ vorhanden } & \multicolumn{3}{c}{ nicht vorhanden } \\
\cline { 2 - 7 } & $\mathbf{N}$ & Mittelwert & SD & N & Mittelwert & SD \\
\hline Gehphase ( 2 Minuten) & & & & & \\
\hline mittlere HR & 57 & 98,49 & 17,35 & 359 & 105,45 & 17,14 \\
\hline Ln SDNN & 57 & 2,89 & 0,62 & 359 & 2,80 & 0,54 \\
\hline Ln rMSSD & 57 & 2,89 & 0,80 & 359 & 2,75 & 0,83 \\
\hline pNN50 & 56 & 0,03 & 0,056 & 358 & 0,03 & 0,06 \\
\hline Ln LF & 57 & 2,59 & 1,49 & 359 & 2,26 & 1,33 \\
\hline Ln HF & 57 & 2,49 & 1,66 & 359 & 2,17 & 1,60 \\
\hline Ln Total & 57 & 4,18 & 1,21 & 359 & 3,86 & 1,18 \\
\hline Ln LF/HF & 57 & 0,04 & 0,50 & 359 & 0,04 & 0,48 \\
\hline
\end{tabular}

Taktatmungsphase (5 Minuten)

\begin{tabular}{lllllll}
\hline mittlere HR & 58 & 71,88 & 13,51 & 378 & 73,94 & 12,50 \\
\hline Ln SDNN & 58 & 3,59 & 0,58 & 376 & 3,52 & 0,51 \\
\hline Ln rMSSD & 58 & 3,20 & 0,83 & 378 & 3,08 & 0,78 \\
\hline pNN50 & 58 & 0,06 & 0,07 & 376 & 0,05 & 0,08 \\
\hline Ln LF & 58 & 5,11 & 1,14 & 377 & 5,22 & 1,13 \\
\hline Ln HF & 58 & 4,06 & 1,35 & 378 & 3,82 & 1,38 \\
\hline Ln Total & 58 & 6,67 & 1,10 & 371 & 6,65 & 0,98 \\
\hline Ln LF/HF & 58 & 0,45 & 0,46 & 377 & 0,61 & 0,44
\end{tabular}

Ruhephase (5 Minuten)

\begin{tabular}{lllllll}
\hline mittlere HR & 57 & 70,92 & 13,19 & 367 & 72,78 & 12,10 \\
\hline Ln SDNN & 57 & 3,38 & 0,53 & 365 & 3,39 & 0,46 \\
\hline Ln rMSSD & 57 & 2,82 & 0,93 & 367 & 2,88 & 0,78 \\
\hline pNN50 & 57 & 0,03 & 0,05 & 367 & 0,03 & 0,05 \\
\hline Ln LF & 57 & 4,71 & 1,32 & 367 & 4,89 & 1,20 \\
\hline Ln HF & 57 & 3,61 & 1,36 & 367 & 3,36 & 1,34 \\
\hline Ln Total & 57 & 6,03 & 1,13 & 366 & 6,02 & 0,96 \\
\hline Ln LF/HF & 56 & 0,49 & 0,44 & 367 & 0,67 & 0,43
\end{tabular}

Tabelle 29 HR- und HRV-Mittelwerte nach Gruppen anhand der HADS „Depressivität vorhanden“ und „Depressivität nicht vorhanden.“ 


\subsubsection{Angst}

\begin{tabular}{ccccccc}
\hline \multirow{2}{*}{ Angst } & \multicolumn{3}{c}{ vorhanden } & \multicolumn{3}{c}{ nicht vorhanden } \\
\cline { 2 - 7 } & $\mathbf{N}$ & Mittelwert & SD & N & Mittelwert & SD \\
\hline Gehphase ( 2 Minuten) & & & & & \\
\hline mittlere HR & 71 & 103,84 & 16,94 & 346 & 104,66 & 17,39 \\
\hline Ln SDNN & 71 & 2,88 & 0,59 & 346 & 2,80 & 0,54 \\
\hline Ln rMSSD & 71 & 2,85 & 0,82 & 346 & 2,75 & 0,83 \\
\hline pNN50 & 70 & 0,04 & 0,06 & 345 & 0,03 & 0,05 \\
\hline Ln LF & 71 & 2,43 & 1,35 & 346 & 2,27 & 1,36 \\
\hline Ln HF & 71 & 2,45 & 1,58 & 346 & 2,16 & 1,61 \\
\hline Ln Total & 71 & 4,06 & 1,16 & 346 & 3,87 & 1,20 \\
\hline Ln LF/HF & 71 & $-0,01$ & 0,52 & 346 & 0,05 & 0,48 \\
\hline
\end{tabular}

Taktatmungsphase (5 Minuten)

\begin{tabular}{ccccccc}
\hline mittlere HR & 77 & 74,49 & 14,48 & 360 & 73,50 & 12,21 \\
\hline Ln SDNN & 77 & 3,49 & 0,51 & 358 & 3,54 & 0,52 \\
\hline Ln rMSSD & 77 & 3,06 & 0,77 & 360 & 3,11 & 0,79 \\
\hline pNN50 & 77 & 0,05 & 0,07 & 358 & 0,05 & 0,08 \\
\hline Ln LF & 77 & 5,13 & 1,02 & 359 & 5,22 & 1,16 \\
\hline Ln HF & 77 & 3,7 & 1,34 & 360 & 3,87 & 1,39 \\
\hline Ln Total & 77 & 6,55 & 0,90 & 353 & 6,67 & 1,02 \\
\hline Ln LF/HF & 77 & 0,60 & 0,45 & 359 & 0,59 & 0,45 \\
\hline
\end{tabular}

Ruhephase (5 Minuten)

\begin{tabular}{ccccccc}
\hline mittlere HR & 76 & 73,82 & 13,85 & 349 & 72,26 & 11,87 \\
\hline Ln SDNN & 76 & 3,33 & 0,51 & 347 & 3,40 & 0,46 \\
\hline Ln rMSSD & 76 & 2,79 & 0,91 & 349 & 2,89 & 0,78 \\
\hline pNN50 & 76 & 0,03 & 0,06 & 349 & 0,03 & 0,05 \\
\hline Ln LF & 76 & 4,75 & 1,13 & 349 & 4,88 & 1,24 \\
\hline Ln HF & 76 & 3,38 & 1,37 & 349 & 3,39 & 1,34 \\
\hline Ln Total & 76 & 5,93 & 0,94 & 348 & 6,03 & 0,99 \\
\hline Ln LF/HF & 76 & 0,59 & 0,48 & 348 & 0,65 & 0,43 \\
\hline
\end{tabular}

Tabelle 30 HR- und HRV-Mittelwerte nach Gruppen anhand der HADS „Angst vorhanden“ und „Angst nicht vorhanden.“ 


\subsubsection{Vitale Erschöpfung}

\begin{tabular}{ccccccc}
\hline \multirow{2}{*}{$\begin{array}{c}\text { Vitale } \\
\text { Erschöpfung }\end{array}$} & \multicolumn{3}{c}{ vorhanden } & \multicolumn{3}{c}{ nicht vorhanden } \\
\cline { 2 - 7 } Gehphase ( 2 Minuten) & Mittelwert & SD & N & Mittelwert & SD \\
\hline mittlere HR & 83 & 102,31 & 17,54 & 298 & 105,56 & 17,50 \\
\hline Ln SDNN & 83 & 2,86 & 0,59 & 298 & 2,79 & 0,54 \\
\hline Ln rMSSD & 83 & 2,84 & 0,82 & 298 & 2,73 & 0,83 \\
\hline pNN50 & 83 & 0,03 & 0,04 & 297 & 0,03 & 0,06 \\
\hline Ln LF & 83 & 2,43 & 1,26 & 298 & 2,25 & 1,40 \\
\hline Ln HF & 83 & 2,28 & 1,46 & 298 & 2,17 & 1,65 \\
\hline Ln Total & 83 & 3,95 & 1,08 & 298 & 3,86 & 1,23 \\
\hline Ln LF/HF & 83 & 0,07 & 0,46 & 298 & 0,03 & 0,48 \\
\hline
\end{tabular}

Taktatmungsphase (5 Minuten)

\begin{tabular}{ccccccc}
\hline mittlere HR & 85 & 73,95 & 14,18 & 317 & 73,40 & 12,18 \\
\hline Ln SDNN & 85 & 3,56 & 0,56 & 316 & 3,54 & 0,50 \\
\hline Ln rMSSD & 85 & 3,13 & 0,82 & 317 & 3,09 & 0,76 \\
\hline pNN50 & 85 & 0,05 & 0,07 & 315 & 0,05 & 0,08 \\
\hline Ln LF & 85 & 5,08 & 1,22 & 316 & 5,27 & 1,09 \\
\hline Ln HF & 85 & 3,90 & 1,30 & 317 & 3,86 & 1,40 \\
\hline Ln Total & 84 & 6,70 & 1,06 & 312 & 6,67 & 0,98 \\
\hline Ln LF/HF & 85 & 0,51 & 0,40 & 316 & 0,62 & 0,46 \\
\hline
\end{tabular}

Ruhephase (5 Minuten)

\begin{tabular}{ccccccc}
\hline mittlere HR & 86 & 73,08 & 13,21 & 306 & 72,17 & 11,91 \\
\hline Ln SDNN & 85 & 3,39 & 0,48 & 305 & 3,40 & 0,47 \\
\hline Ln rMSSD & 86 & 2,88 & 0,86 & 306 & 2,87 & 0,78 \\
\hline pNN50 & 86 & 0,03 & 0,05 & 306 & 0,03 & 0,05 \\
\hline Ln LF & 86 & 4,71 & 1,30 & 306 & 4,94 & 1,20 \\
\hline Ln HF & 86 & 3,50 & 1,33 & 306 & 3,36 & 1,31 \\
\hline Ln Total & 86 & 6,01 & 1,05 & 306 & 6,06 & 0,97 \\
\hline Ln LF/HF & 86 & 0,53 & 0,44 & 305 & 0,69 & 0,43
\end{tabular}

Tabelle 31 HR- und HRV-Mittelwerte nach Gruppen anhand MQ „Vitale Erschöpfung vorhanden“ und „Vitale Erschöpfung nicht vorhanden.“ 


\subsubsection{Körperliche Funktionsfähigkeit}

\begin{tabular}{lclllll}
\hline \multirow{2}{*}{$\begin{array}{c}\text { körperliche } \\
\text { Funktionsfähigkeit }\end{array}$} & $\mathbf{N}$ & Mittelwert & SD & N & Mittelwert & SD \\
\cline { 2 - 7 } Gehphase (2 Minuten) & & & & & \\
\hline mittlere HR & 75 & 97,88 & 15,94 & 339 & 106,06 & 17,69 \\
\hline Ln SDNN & 75 & 2,87 & 0,54 & 339 & 2,80 & 0,55 \\
\hline Ln rMSSD & 75 & 2,94 & 0,79 & 339 & 2,73 & 0,84 \\
\hline pNN50 & 74 & 0,03 & 0,05 & 338 & 0,03 & 0,06 \\
\hline Ln LF & 75 & 2,32 & 1,33 & 339 & 2,29 & 1,36 \\
\hline Ln HF & 75 & 2,55 & 1,51 & 339 & 2,15 & 1,64 \\
\hline Ln Total & 75 & 3,99 & 1,11 & 339 & 3,88 & 1,21 \\
\hline Ln LF/HF & 75 & $-0,10$ & 0,47 & 339 & 0,06 & 0,48 \\
\hline
\end{tabular}

Taktatmungsphase (5 Minuten)

\begin{tabular}{lllllll}
\hline mittlere HR & 76 & 72,55 & 12,94 & 357 & 73,66 & 12,71 \\
\hline Ln SDNN & 76 & 3,49 & 0,62 & 356 & 3,54 & 0,49 \\
\hline Ln rMSSD & 76 & 3,16 & 0,86 & 357 & 3,08 & 0,76 \\
\hline pNN50 & 75 & 0,05 & 0,07 & 355 & 0,05 & 0,08 \\
\hline Ln LF & 76 & 4,83 & 1,22 & 356 & 5,29 & 1,08 \\
\hline Ln HF & 76 & 3,95 & 1,44 & 357 & 3,85 & 1,37 \\
\hline Ln Total & 75 & 6,45 & 1,14 & 352 & 6,71 & 0,95 \\
\hline Ln LF/HF & 76 & 0,38 & 0,51 & 356 & 0,63 & 0,44
\end{tabular}

Ruhephase (5 Minuten)

\begin{tabular}{lllllll}
\hline mittlere HR & 75 & 71,79 & 11,51 & 347 & 72,53 & 12,51 \\
\hline Ln SDNN & 75 & 3,40 & 0,54 & 345 & 3,38 & 0,45 \\
\hline Ln rMSSD & 75 & 2,99 & 0,86 & 347 & 2,84 & 0,79 \\
\hline pNN50 & 75 & 0,02 & 0,04 & 347 & 0,03 & 0,06 \\
\hline Ln LF & 75 & 4,55 & 1,43 & 347 & 4,93 & 1,15 \\
\hline Ln HF & 75 & 3,52 & 1,52 & 347 & 3,36 & 1,28 \\
\hline Ln Total & 75 & 5,92 & 1,26 & 347 & 6,04 & 0,92 \\
\hline Ln LF/HF & 74 & 0,46 & 0,45 & 347 & 0,68 & 0,43
\end{tabular}

Tabelle 32 HR- und HRV-Mittelwerte nach Gruppen anhand SF-36 „körperliche Funktionsfähigkeit niedrig“ beziehungsweise ,körperliche Funktionsfähigkeit hoch.“ 


\subsection{T-Testung bearbeiteter und unbearbeiteter EKGs}

Zur Prüfung des Einflusses von zeilenweisen Ausschlüssen des EKGs auf die HR und die HRV-Parameter wurde eine T-Testung mit getrennten Varianzen gerechnet.

\begin{tabular}{|c|c|c|c|}
\hline & 1 gegen 2 & 2 gegen 3 & 1 gegen 3 \\
\hline mittlere HR & & $* \star$ & ** \\
\hline SDNN & & * & 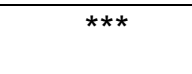 \\
\hline rMSSD & & * & $\star \star \star *$ \\
\hline pNN50 & & & ** \\
\hline LF & & * & $* \star$ \\
\hline HF & & & $* * *$ \\
\hline Total & & & $* \star$ \\
\hline LF/HF & ** & & ** \\
\hline
\end{tabular}

Tabelle 33 T-Testung nach Gruppen: Gruppe 1: kein zeilenweiser Ausschluss; Gruppe 2: Ausschlüsse, aber nicht in EKG-Zeile 1; Gruppe 3: Ausschlüsse in EKG-Zeile 1 und ggf. weiteren EKG-Zeilen. Nur signifikante Mittelwertsunterschiede mit Signifikanzniveau markiert durch Sterne angegeben. 


\section{Abkürzungsverzeichnis}

AA: absolute Arrhythmie

ACC: American College of Cardiology

AHA: American Heart Association

ANP: atriales natriuretisches Peptid

ANS: autonomes Nervensystem

BIT: ,binary digit”

BMI: ,body mass index”

BNP: B-type natriuretisches Peptid

Bpm: „,beats per minute“: Herzschläge pro Minute= Herzfrequenz

DM: Diabetes mellitus

dpw: „drinks per week”: 1 „drink” = 14g reiner Alkohol (National institute on alcohol abuse and alcoholism)

DS 14: Type D Scale-14: Fragebogen zur Typ D-Persönlichkeit

E: E-Welle: passive frühdiastolische Füllung des LV.

$\mathrm{e}^{\prime}:$ Gewebedoppler am medialen Mitralanulus ( $\left.\mathrm{cm} / \mathrm{s}\right)$

E/A: Verhältnis Geschwindigkeit Ventrikel- zu Vorhoffüllung

EKG: Elektrokardiographie

ESSI: ENRICHD Social Support Inventory: Fragebogen zur sozialen Unterstützung

FKV: Freiburger Fragebogen zur Krankheitsverarbeitung

Geh: Gehphase

GKE: Fragebogen zur Kompetenzerwartung und Selbstwirksamkeitserwartung

HADS: Hospital Anxiety and Depression Scale

HADS-D: Hospital Anxiety and Depression Scale, deutsche Version.

HADS-D/A: Subskala Angst der deutschen Version der HADS

HADS-D/D: Subskala Depression der deutschen Version der HADS

HF: „high frequency”: hochfrequente Komponente der HRV

HI: Herzinsuffizienz

HR: „heart rate“, Herzfrequenz

HRV: „,heart rate variability“, Herzfrequenzvariabilität

HTN: Hypertonus

Hz: Hertz

ICD: International Statistical Classification of Diseases and Related Health Problems 
KHK: koronare Herzkrankheit

KKSL: Leipziger Koordinierungszentrums für klinische Studien

KNHI: Kompetenznetz Herzinsuffizienz

LF: „low Frequency“: niederfrequente Komponente der HRV

Ln: Logarithmus naturalis zur Basis e

LQ: Lebensqualität

LVEF: linksventrikuläre Ejektionsfraktion

LZ-EKG: Langzeit-Elektrokardiographie

m: Meter

MD: missing Data

MI: „myocardial infarction“: Herzinfarkt

Min: Minuten

MMPI-Z-Skala: Zynismusskala des Minnesota Personality Inventory-2

MQ: Maastricht-Fragebogen

ms: Millisekunden

MTA: medizinisch-technischer Assistent

$\mathrm{N}$ : Normalschlag

NN: Abstand von Normalschlag zu Normalschlag

NP: natriuretische Peptide

NT-pro-BNP: amino-terminales Fragment des Prohormons von BNP

NYHA (-Klasse): Schweregrad der chronischen Herzinsuffizienz nach der New York Heart Association

pAVK: periphere arterielle Verschlusskrankheit

PHQ-D: Prime MD Patient Health Questionnaire, deutsche Version: Fragebogen zur Erfassung von somatoformen, depressiven Störungen sowie Panik- und Essstörungen sowie Alkoholmissbrauch.

pNN50: Prozent der NN-Intervalldifferenzen, die $50 \mathrm{~ms}$ überschreiten

rMSSD: „root mean square of standard deviation“: Standardabweichung des Mittels der quadrierten Differenzen benachbarter RR-Intervalle

RR-Intervalle: Abstand zwischen zwei R-Zacken im EKG

RSA: respiratorische Sinusarrhythmie

RSQ: Beziehungsfragebogen

RU: Ruhephase

S/D: Systolisch/Diastolisch 
SD: „standard deviation“; Standardabweichung

SDNN: SD aller NN Intervalle

SF-36: Short Form Health Survey

TA: Taktatmung

UMG: Universitätsmedizin Göttingen

VE: Vitale Erschöpfung

VES: Ventrikuläre Extrasystolen

VLF: ,very low frequency“: sehr niederfrequente Komponente der HRV 


\section{Literaturverzeichnis}

(Aaronson et al. 1992) $=$ Aaronson NK, Acquadro C, Alonso J, Apolone G, Bucquet D, Bullinger M, Bungay K, Fukuhara S, Gandek B, Keller S et al. (1992): International quality of life assessment (IQOLA) project. Qual Life Res 1, 349-351.

$($ Agelink et al. 2001)= Agelink MW, Malessa R, Baumann B, Majewski T, Akila F, Zeit T, Ziegler D (2001): Standardized tests of heart rate variability: normal ranges obtained from 309 healthy humans, and effects of age, gender, and heart rate. Clin Auton Res 11, 99-108.

(Alonso et al. 2004)= Alonso J, Ferrer M, Gandek B, Ware JEJr, Aaronson NK, Mosconi P, Rasmussen NK, Bullinger M, Fukuhara S et al. and the IQOLA Project Group (2004): Healthrelated quality of life associated with chronic conditions in eigth countries: Results from the International Quality of Life Assessment (IQOLA) Project. Qual Life Res 13, 283-298.

(Angermann und Ertl 2004)= Angermann CE, Ertl G (2004): Diagnostik der Herzinsuffizienz. Med Welt 11-12, 359-366.

(Angermann et al. 2011)= Angermann CE, Gelbrich G, Störk S, Schowalter M, Deckert J, Ertl G, Faller H (2011): Somatic correlates of comorbid major depression in patients with systolic heart failure. Int J Cardiol 147(1), 66-73. (Abstract)

(Appels 2004)= Appels A (2004): Exhaustion and coronary heart disease: the history of a scientific quest. Patient Educ Couns 55, 223-229.

(Appels et al. 1987)= Appels A, Höppener P, Mulder P (1987): A questionnaire to assess premonitory symptoms of myokardial infarction. Int J Cardiol 17(1), 15-24.

(Appels et al. 2000)= Appels A, Kop WJ, Schouten E (2000): The Nature of the Depressive Symptomatology Preceding Myocardial Infarction. Behav Med 26(2), 86-89. 
(Battes et al. 2011)= Battes LC, Pedersen SS, Oemrawsingh RM, van Geuns RJ, Al Amri I, Regar E, de Jaegere PP, Serruys P, van Domburg RT (2011): Beta blocker therapy is associated with reduced depressive symptoms 12 months post percutaneous coronary intervention. $\mathrm{J}$ Affect Disord 2011 Oct 25 [Epub ahead of print]. (Abstract)

(Bernardi et al. 2000)= Bernardi L, Wdowczyk-Szulc J, Valenti C, Castoldi S, Passino C, Spadacini G, Sleight P (2000): Effects of controlled breathing, mental activity and mental stress with or withour verbalization on heart rate variability. J Am Coll Cardiol 35(6), $1462-$ 1469.

(Biro et al. 1996)= Bíró É, Tóth G, Telegedy G (1996): Effect of receptor blockers on brain natriuretic peptide and C-type natriuretic peptide caused anxiolytic state in rats. Neuropepti$\operatorname{des} \underline{30(1)}, 59-65$.

(Bremecker 2010)= Bremecker K: Einfluss einer leitliniengerechten Behandlung des arteriellen Hypertonus auf echokardiographische Parameter der diastolischen Funktion. Med. Diss. Göttingen 2010.

(Bullinger 1995)= Bullinger M (1995): German Translation and Psychometric Testing of the SF-36 Health Survey: Preliminary Relults from the IQOLA Project. Soc Sci Med 41 (10), 1359-1366.

(Bullinger 1997)= Bullinger M. Gesundheitsbezogene Lebensqualität und subjektive Gesundheit: Überblick über den Stand der Forschung zu einem neuen Evaluationskriterum in der Medizin. Psychother Psychosom Med Psychol 47(3-4), 76-91.

(Bullinger 2000)= Bullinger M (2000): Erfassung der gesundheitsbezogenen Lebensqualität mit dem SF-36-Health Survey. Bundesgesundheitsblatt Gesundheitsforschung Gesundheitsschutz $\underline{43,190-197 .}$

(Bullinger und Kirchberger 1998)= Bullinger M, Kirchberger I: SF-36 Fragebogen zum Gesundheitszustand. Hogrefe Verlag für Psychologie, Göttingen 1998. 
(Bunevicius et al. 2006)= Bunevicius R, Varonbeckas G, Prange AJ, Hinderliter AL, Gintauskiene V, Girdler SS (2006): Depression and Thyroid Axis Function in Coronary Artery Disease: Impact of Cardiac Impairment and Gender. Clin Cardiol 29(4), 170-174.

(Carney und Freedland 2009)=Carney RM, Freedland KE 2009: Depression and heart rate variability in patients with coronary heart disease. Cleve Clin J Med 76 (Suppl 2), S13-17.

(Carney et al. 2001)= Carney RM, Blumenthal JA, Stein PK, Watkins L, Catellier D, Berkman LF, Czaijkowski SM, O’Connor C, Stone PH, Freedland KE (2001): Depression, Heart Rate Variability, and Acute Myocardial Infarction. Circulation 104(17), 2024-2028.

(Carney et al. 2002)= Carney RM, Freedland KE, Miller GE, Jaffe AS (2002): Depression as a risk factor for cardiac mortality and morbidity: A review of potential mechanisms. J Psychosom Res 53(4), 897-902.

(Crawford et al. 1999)= Crawford MH, Bernstein SJ, Deedwania PC, DiMarco JP, Ferrick KJ, Garson AJr, Green LA, Greene HL, Silka MJ, Stone PH et al. (1999): ACC/AHA Guidelines for Ambulatory Electrocardiography. Circulation 100(8), 886-893.

(Davydov et al. 2007)= Davydov DM, Shapiro D, Cook IA, Goldstein I (2007): Baroreflex mechanisms in major depression. Prog Neuropsychopharmacol Biol Psychiatry 31(1), 164177.

(Dietz 2004)= Dietz R (2004): Das Kompetenznetz Herzinsuffizienz. Med Welt 11-12, 4.

(Einvik et al. 2011)= Einvik G, Hrubos-Strøm H, Randby A, Nordhus IH, Somers VK, Omland T, Dammen T (2011): Major Depressive Disorder, Anxiety Disorders, and Cardiac Biomarkers in Subjects at High Risk of Obstructive Sleep Apnea. Psychosom Med 73(5), 378384.

(Enright et al. 2003)= Enright PL, McBurnie MA, Bittner V, Tracy RP, McNamara R, Arnold A, Newman AB (2003): The 6-min Walk Test: A Quick Measure of Functional Status in Elderly Adults. Chest 123(2), 387-398. 
$($ Friedman 2007) $=$ Friedman BH (2007): An autonomic flexibility-neurovisceral integration model of anxiety and cardiac vagal tone. Biol Psychol 74(2), 185-199.

$($ Frenneaux 2004)= Frenneaux MP (2004): Autonomic changes in patients with heart failure and in post-myocardial infarction patients. Heart $\underline{90(11)}, 1248-1255$.

(Gaburro et al. 2011)= Gaburro S, Stiedl O, Sartori SB, Landgraf R, Singewald N (2011): A mouse model of high trait anxiety shows reduced heart rate variability that can be reversed by anxiolytic drug treatment. Int J Neuropsychopharmacol 14(10), 1341-1355.

$($ Giardino et al. 2010)= Giardino ND, Curtis JL, Andrei AC, Fan VS, Benditt JO, Lyubkin M, Naunheim K, Criner G, Make B et al. the NETT Research Group (2010): Anxiety is associated with diminished exercise performance and quality of life in severe emphysema: a crosssectional study. Respir Res $\underline{11}, 29$.

(Golenhofen und Hildebrandt 1958)= Golenhofen K, Hildebrandt G (1958): Die Beziehung des Blutdruckrythmus zu Atmung und peripherer Durchblutung. Pflugers Arch 267(1), 27-45.

$($ Gorkin et al. 1993)= Gorkin L, Norvell NK, Rosen RC, Charles E, Shumaker SA, McIntyre KM, Capone RJ, Kostis J, Niaura R et al. for the SOLVD Investigators (1993): Assessment of Quality of Life as Observed from the Baseline Data of the Studies of Left Ventricular Dysfunction (SOLVD) Trial Quality-of-Life Substudy. Am J Cardiol 71(12), 1069-1073.

(Grippo et al. 2002)= Grippo AJ, Moffitt JA, Johnson AK (2002): Cardiovascular alterations and autonomic imbalance in an experimental model of depression. Am J Physiol Regul Integr Comp Physiol 282(5),R1333-1341.

(Haass et al. 2000)= Haass M, Zugck C, Kübler W (2000): Der 6-Minuten-Gehtest: eine kostengünstige Alternative zur Spiroergometrie bei Patienten mit chronischer Herzinsuffizienz? Z Kardiol 89(2), 72-80.

(Hathaway et al. 2000)= Hathaway DK, Wicks MN, Cashion AK, Cowan PA, Milstead EJ, Gaber AO (2000): Posttransplant improvement in heart rate variability correlates with improved quality of life. West J Nurs Res 22(6),749-768. (Abstract) 
(Hennersdorf und Strauer 2002)= Hennersdorf MG, Strauer BE (2002): Autonome Dysfunktion bei Herzerkrankungen und Diabetes Mellitus. Monitoring und Diagnostik. Internist (Berl) 43(9): 1065- 1075 .

(Herring et al. 2001)= Herring N, Zaman JA, Paterson DJ (2001): Natriuretic peptides like NO facilitate cardiac vagal neurotransmission and bradycardia via a cGMP pathway. Am J Physiol Heart Circ Physiol 281(6), H2318-H2327.

(Herrmann 1997)= Herrmann C (1997): International experiences with the Hospital Anxiety and Depression Scale-a review of validation data and clinical results. J Psychosom Res $\underline{42(1)}$, $17-41$.

(Herrmann und Buss 1994)= Herrmann C, Buss U (1994): Vorstellung und Validierung einer deutschen Version der „Hospital Anxiety und Depression Scale,,(HAD-Skala): Ein Fragebogen zur Erfassung des psychischen Befindens bei Patienten mit körperlichen Beschwerden. Diagnostica 40 (2), 143-154.

(Herrmann et al. 2000)= Herrmann C, Brand-Driehorst S, Buss U, Rüger U (2000): Effects of anxiety and depression on 5-year mortality in 5057 patients referred for exercise testing. $\mathbf{J}$ Psychosom Res 48(4-5), 455-462.

(Herrmann-Lingen 2003)= Herrmann-Lingen C (2003): Zur Bedeutung depressiver Störungen für die somatische Medizin. Z Psychosom Med Psychother 49(4): 391-407.

(Herrmann-Lingen et al. 2003)= Herrmann-Lingen C, Binder L, Klinge M, Sander J, Schenker W, Beyermann B, von Lewinski D, Pieske B (2003): High Plasma Levels of N-Terminal Pro-Atrial Natriuretic Peptide Associated With Low Anxiety in Severe Heart Failure. Psychosom Med 65(4): 517-522.

(Herrmann-Lingen et al. 2004)= Herrmann-Lingen C, Rautenstrauch N, Binder L, Lürs C, Wetzel D, Pouwels C, Kochen MM, Pieske B (2004): Association of NT-Pro-BNP With Quality of Life and Anxiety in Men and Women With CHF Risk Faktors. J Psychosom Res $\underline{56}, 611$. 
(Herrmann-Lingen et al. 2005)= Herrmann-Lingen C, Buss U, Snaith RP: HADS-D. Hospital and Depression Scale-Deutsche Version. Hans Huber Verlag, Bern 2005.

(Hoogwegt et al. 2012)= Hoogwegt MT, Kupper N, Theuns DA, Jordaens L, Pedersen SS (2012): Beta-blocker therapy is not associated with symptoms of depression and anxiety in patients receiving an implantable cardioverter-defibrillator. Europace 14(1), 74-80. (Abstract)

(Januzzi et al. 2000)= Januzzi JL, Stern TA, Pasternak RC, DeSanctis RW (2000): The Influence of Anxiety and Depression on Outcomes of Patients With Coronary Artery Disease. Arch Intern Med 160(13): 1913-1921.

(Juenger et al. 2002)= Juenger J, Schellberg D, Kraemer S, Haunstetter A, Zugck C, Herzog W, Haass M (2002): Health related quality of life in patients with congestive heart failure: comparison with other chronic diseases and relation to functional variables. Heart $\underline{87(3), 235-}$ 241.

(Kemp et al. 2010)= Kemp AH, Quintana DS, Gray MA, Felmingham KL, Brown K, Gatt JM (2010): Impact of Depression and Antidepressant Treatment on Heart Rate Variability: A Review and Meta-Analysis. Biol Psychiatry $\underline{67(11)}$, 1067-1074.

(Klein 1974)= Klein DF(1974): Endogenomorphic depression. Arch Gen Psychiatry $\underline{31(4)}$, 447-454.

(Koertge 2003)= Koertge J: Vital Exhaustion and Coronary Artery Disease in Women: Biological Correlates and Behavioral Intervention. Med. Diss. Stockholm 2003.

(Kop 1999)= Kop WJ (1999): Chronic and Acute Psychological Risk Factors for Clinical Manifestations of Coronary Artery Disease. Psychosom Med 61(4), 476-487.

(Ladwig et al. 2008)= Ladwig KH, Lederbogen F, Völler H, Albus C, Herrmann-Lingen C, Jordan J, Köllner V, Jünger J, Lange H, Fritzsche K (2008): Positionspapier zur Bedeutung von psychosozialen Faktoren in der Kardiologie. Kardiologe 2, 274- 287. 
(Laux et al. 1981)= Laux L, Glanzmann P, Schaffner P, Spielberger CD: Das State-TraitAngstinventar - Theroretische Grundlagen und Handanweisung. Beltz-Verlag, Weinheim 1982.

(Lord und Menz 2002)= Lord SR, Menz HB (2002): Physiologic, Psychologic, and Health Predictors of 6-Minute Walk Performance in Older People. Arch Phys Med Rehabil 83(7), 907-911.

(MacMahon und Lip 2002) = MacMahon KMA, Lip GYH (2002): Psychological Factors in Heart Failure: A Review of Literature. Arch Intern Med 162(5), 509-516.

(Mair et al. 2001)= Mair J, Hammerer-Lercher A, Puschendorf B (2001): Review: The Impact of Cardiac Natriuretic Peptide Determination on the Diagnosis and Management of Heart Failure. Clin Chem Lab Med 39(7), 571-588.

(Malik 1998)= Malik M (1998): Heart rate variability. Curr Opin Cardiol 13(1), 36-44.

(McHorney et al. 1993)= McHorney CA, Ware JE, Raczek AE (1993): The MOS 36-Item Short-Form Health Survey (SF-36): II. Psychometric and Clinical Tests of Validity in Measuring Physical and Mental Health Constructs. Med Care 31(3), 247-263.

(McHorney et al. 1994)= Mc Horney CA, Ware JE Jr, Lu JF, Sherbourne CD (1994): The MOS 36-item Short-Form Health Survey(SF-36): III. Tests of data quality, scaling assumptions, and reliability across diverse patient groups. Med Care $\underline{32(1)}$, 40-66. (Abstract)

(Medilog AR 12 Primer 2002)= Medilog AR12 Primer : Technical Training. TOM Medical $\mathrm{GmbH}$, Austria 2002.

(Medilog Darwin Holter Analysis 2004)= Medilog Darwin Holter Analysis: Clinical Guide: Signal Theory Basics. TOM Medical Handlungs GmbH. Edition 1.0, Austria 2004.

(Medilog Darwin Holter Analysis 2006a)= Medilog Darwin Holter Analysis: Gebrauchsanweisung, Benutzerhandbuch. TOM Medical Entwicklungs GmbH. Edition 1.1( für Medilog Darwin Version 1.5), Austria 2006. 
(Medilog Darwin Holter Analysis 2006b)= Medilog Darwin Holter Analysis: Gebrauchsanweisung: Referenz Handbuch. TOM Medical Entwicklungs GmbH. Edition 1.3 (für Medilog Darwin Version 1.5.), Austria 2006.

(Meyer C et al. 2000)= Meyer $\underline{\text { }}$, Rumpf HJ, Hapke U, Dilling H, John U (2000): Lebenszeitprävalenz psychischer Störungen in der erwachsenen Allgemeinbevölkerung. Ergebnisse der TACOS-Studie. Nervenarzt $\underline{71(7)}$, 535-542.

(Meyer T et al 2010)= Meyer $\underline{T}$, Buss U, Herrmann-Lingen C (2010): Role of Cardiac Disease Severity in the Predictive Value of Anxiety for All-Cause Mortality. Psychosom Med $\underline{72(1)}, 9-15$.

(Mueck-Weymann et al. 2002)= Mueck-Weymann M, Moesler T, Joraschky P, Rebensburg M, Agelink MW (2002): Depression Modulates Autonomic Cardiac Control: A Physiopsychological Pathway Linking Depression and Mortality? German J Psychiatry $\underline{5}$, 67-69.

(Mueller et al. 2004)= Mueller T, Gegenhuber A, Poelz W, Haltmayer M (2004): Head-tohead comparison of the diagnostic utility of BNP and NT-pro-BNP in symptomatic and asymptomatic structural heart disease. Clin Chim Acta 341(1-2), 41-48.

(Nahshoni et al. 2004)= Nahshoni E, Aravot D, Aizenberg D, Sigler M, Zalsman G, Strasberg B, Imbar S, Adler E, Weizman A (2004): Heart Rate Variability in Patients With Major Depression. Psychosomatics 45(2), 129-134.

(Niederl 2008)= Thomas Niederl: FFT-Breite und andere technische Details bezüglich des Medilog Darwin AR 12 und der dazugehörigen Software in: Konversation mittels E-mail am 21.04.08.

(Pagani et al. 1997)= Pagani M, Montano N, Porta A, Malliani A, Abboud FM, Birkett C, Somers VK (1997): Relationship Between Spectral Components of Cardiovascular Variabilities and Direct Measures of Muscle Sympathetic Nerve Activity in Humans. Circulation 95(6), 1441-1448. 
(Paulus et al. 2007)= Paulus WJ, Tschöpe C, Sanderson JE, Rusconi C, Flachskampf FA, Rademakers FE, Marino P, Smiseth OA, De Keulenaer G, Leite-Moreira AF et al. (2007): How to diagnose diastolic heart failure: a consensus statement on the diagnosis of heart failure with normal left ventrikular ejection fraction by the Heart Failure and Echocardiography Association of the European Society of Cardiology. Eur Heart J 28(20), 2539-2550.

(Parissis et al. 2008)= Parissis JT, Nikolaou M, Farmakis D, Bistola V, Paraskevaidis IA, Adamopoulos S, Filippatos G, Kremastinos DT (2008): Clinical and prognostic implications of self-rating depression scales and plasma B-type natriuretic peptide in hospitalised patients with chronic heart failure. Heart 94(5), 585-589.

(Rajendra Acharya et al. 2006)= Rajendra Acharya U, Paul Joseph K, Kannathal N, Lim CM, Suri JS (2006): Heart rate variability: a review. Med Biol Eng Comput 44(12), 1031-1051.

(Rauchhaus 2004)= Rauchhaus M (2004): Pathophysiologie der Herzinsuffizienz. Med Welt $\underline{55,380-385 .}$

(Remme und Swedberg 2001)= Remme WJ, Swedberg K (2001): Task Force for the Diagnosis and Treatment of Chronic Heart Failure, European Society of Cardiology. Eur Heart J 22(17), 1527-1560.

(Robert-Koch-Institut 2006) $=$ Robert Koch-Institut in Zusammenarbeit mit dem Statistischen Bundesamt: Gesundheitsberichterstattung des Bundes: Gesundheit in Deutschland 2006. Robert-Koch-Institut, Berlin 2006.

(Roest et al. 2010a) $=$ Roest AM, Martens EJ, Denollet J, DeJonge P (2010): Prognostic Association of Anxiety Post Myocardial Infarction With Mortality and New Cardiac Events: A Meta-Analysis. Psychosom Med 72(6), 563-569.

(Roest et al. 2010b)= Roest AM, Martens EJ, DeJonge P, Denollet J (2010): Anxiety and Risk of Incident Coronary Heart Disease: A Meta-Analysis. J Am Coll Cardiol 56(1), 38-46.

(Rottenberg 2007)= Rottenberg J (2007): Cardiac vagal control in depression: A critical analysis. Biol Psychol 74(2), 200-211. 
(Sayar et al. 2002)= Sayar K, Gülec H,Gökçe M, Ak I (2002): Heart Rate Variability in Depressed Patients. Bull Clin Psychopharmacol 12 (3), 130-133.

(Scheele 2011) $=$ Scheele F: Prävalenz, Risikofaktoren und klinische Ausprägung der systolischen und diastolischen Herzinsuffizienz in einem hausärztlichen Risikokollektiv sowie Wertigkeit echokardiographischer Parameter und natriuretischer Peptide zur Diagnosestellung der diastolischen Herzinsuffizienz. Med. Diss. Göttingen 2011.

(Schmid et al. 2010)= Schmid K, Schönlebe J, Drexler H, Mueck-Weymann M (2010): Associations between overweight, variability in heart rate, and well-being in the young men. Cardiol Young 20 (1), 54-59. (Abstract)

(Sinski et al. 2006)= Sinski M, Lewandowski J, Abramczyk P, Narkiewicz K, Gaciong Z (2006): Why study sympathetic nervous system? J Physiol Pharmacol 57 (suppl 11), 79-92.

(Snaith 1987)= Snaith RP (1987): The concept of mild depression. Br J Psychiatry 150, 387393.

(Statistisches Bundesamt 2007)= Statistisches Bundesamt (2007): Gesundheit: Fallpauschalenbezogene Krankenhausstatistik (DRG-Statistik), Diagnosen und Prozeduren der vollstationären Patienten und Patientinnen in Krankenhäusern 2006; 2007; Fachserie 12, Reihe 6,4.

(Ströhle et al. 2001)= Ströhle A, Kellner M, Holsboer F, Wiedemann K (2001): Anxiolytic Activity of Atrial Natriuretic Peptide in Patients With Panic Disorder. Am J Psychiatry 158(9), 1514-1516.

(Sztajzel 2004)= Sztajzel J (2004): Heart rate variability: a noninvasive electrocardiographic method to measure the autonomic nervous system. Swiss Med Wkly 134(35-36), 514-522.

(Tarlov et al. 1989)= Tarlov AR, Ware JE, Greenfield S, Nelson EC, Perrin E, Zubkoff M (1989): The Medical Outcome Study: An Application of Methods for Monitoring the Results of Medical Care. JAMA 262, 925-930. 
(Task Force of The European Society of Cardiology and The North American Society of Pacing and Electrophysiology 1996)= Task Force of The European Society of Cardiology and The North American Society of Pacing and Electrophysiology (1996): Heart rate variability: Standards of measurement, physiological interpretation and clinical use. Eur Heart J 17(3), 354-381.

(Tentolouris et al. 2008)= Tentolouris N, Argyrakopoulou G, Katsilambros N (2008): Perturbed Autonomic Nervous System Function in Metabolic Syndrome. Neuromolecular Med $\underline{10(3)}, 169-178$.

(Ulvik et al. 2008)= Ulvik B, Nygård O, Hansestad BR, Wentzel-Larsen T, Wahl AK (2008): Associations between disease severity, coping and dimensions of health-related quality of life in patients admitted for elective coronary angiography - a cross sectional study. Health Qual Life Outcomes $\underline{6}, 38$.

(VanGestel et al. 2011)= Van Gestel AJR, Kohler M, Steier J, Teschler S, Russi EW, Teschler H (2011): Cardiac autonomic dysfunction and health-related quality of life in patients with chronic obstructive pulmonary disease. Respirology 16(6), 939-946. (Abstract)

(Ware und Sherbourne 1992)= Ware JE Jr, Sherbourne CD (1992): The MOS 36-item shortform health survey (SF-36): I. Conceptual framework and item selection. Med Care 30(6), 473-483. (Abstract)

(Watanabe et al. 2002) = Watanabe T, Sugiyama Y, Sumi Y, Watanabe M, Takeuchi K, Kobayashi F, Kono K (2002): Effects of Vital Exhaustion on Cardial Autonomic Nervous Function Assessed by Heart Rate Variability in Rest in Middle-Aged Male Workers. Int J Behav Med 9(1), 68-75.

(Węgrzynowska-Teodorczyk et al. 2010)= Węgrzynowska-Teodorczyk K, Rudzińska E, Jankowska E, Agnieszka Grzesło A, Nowakowska K, Łazorczyk M, Banasiak W, Ponikowski P, Woźniewski M (2010): Determinants of physical fitness in males with systolic heart failure. Kardiol Pol 68(2), 146-154. 
(Williams et al. 2010)= Williams JE, Mosley THJr, Kop WJ, Couper DJ, Welch VL, Rosamond WD (2010): Vital Exhaustion as a Risk Factor for Adverse Cardiac Events (from the Atherosclerosis Risk In Communities [ARIC] Study). Am J Cardiol 105(12), 1661- 1665.

(Yeragani 1995)= Yeragani VK(1995): Heart Rate and Blood Pressure Variability: Implications for Psychiatric Research. Neuropsychobiology 32(4), 182-191.

(Zerssen und Koeller 1976)= Zerssen Dv, Koeller DM: Paranoid-Depressivitäts-Skala, Depressivitäts-Skala - Manual. Beltz-Verlag, Weinheim 1976.

(Ziemssen et al. 2002)= Ziemssen T, Süss M, Reichmann H (2002): Die kardiovaskuläre Funktionsdiagnostik des autonomen Nervensystems-eine Einführung. Neurophysiol Lab $\underline{24(2)}, 57-77$.

(Zigmond und Snaith 1983)= Zigmond AS and Snaith RP (1983): The Hospital Anxiety and Depression Scale. Acta Psychiatr Scand 67(6), 361-370. 


\section{Danksagungen:}

Herrn Prof. Dr. Christoph Herrmann-Lingen für die freundliche Überlassung des Themas und die langjährige hervorragende Betreuung.

Meiner zwischenzeitlichen Betreuerin Dr. Beate Stanske, dem psychosomatischen Statistiktutor Dipl. Psych. Andreas Cordes sowie der statistischen Beratungsstelle der Universität Göttingen für die beratende Unterstützung.

Den Mitarbeitern des Kompetenznetzes insbesondere Prof. Dr. med. Burkert Pieske, Prof. Dr. med. Gerd Hasenfuß, Prof. Dr. med. Michael M. Kochen, Dr. Rolf Wachter, Dr. Sibylle Kleta, Dipl. Psych. Birgit Büchner, Angela Krull und Christine Thomas.

Meinen Mitdoktoranden im Kompetenznetz für die gute Zusammenarbeit. Besonders Dr. Kerstin Bremecker, Dr. Frauke Scheele (geb. Oltmanns), Anja Siegert, Iris Kaiser, Cordelia Wilke (geb. Huf) und Sonja Pieper (geb. Schmidt).

Dem Team von Oxford Systems für die Zusammenarbeit bei der Verfeinerung der Software. Hier insbesondere Herrn Niederl und Herrn Fürst.

Den niedergelassenen Kollegen für die Rekrutierung.

Abschließend ein ganz herzlicher Dank an die Patienten, die mit ihrer Bereitschaft zur freiwilligen Teilnahme an dieser Untersuchung diese Arbeit überhaupt erst ermöglicht haben. 